\title{
NARRATIVE IDENTITY: \\ THE CONSTRUCTION OF THE LIFE STORY, AUTOBIOGRAPHICAL REASONING AND PSYCHOLOGICAL FUNCTIONING IN YOUNG ADULTHOOD
}

BY

MEGAN VANESSA BANKS

\begin{abstract}
A thesis
submitted to the Victoria University of Wellington in fulfilment of the requirements for the degree of Doctor of Philosophy
\end{abstract}

Victoria University of Wellington

2013 


\section{Abstract}

According to McAdams' $(1988$; 1993) Life Story Model of Identity, narrative identity is constructed through the development of the life story in adolescence and young adulthood. This theoretical claim has sparked an emerging body of research examining links between the development of the life story and psychological functioning during this developmental period (McLean \& Breen, 2009; McLean, Breen, \& Fournier, 2010; Tavernier \& Willoughby, 2012). The aim of this thesis was to contribute to this emerging body of work by examining the relationship between autobiographical reasoning, the core process through which the life story develops, and psychological functioning in young adulthood. Across four studies, young adults constructed life story narratives of high points, low points and turning points from their life story. These narratives were coded for the presence, and valence, of autobiographical reasoning. Autobiographical reasoning was measured primarily in terms of self-event connections, statements linking an aspect of the narrated event to the young adults' sense of self (McLean \& Fournier, 2008). Autobiographical reasoning valence was measured in terms of self-event connections that described the self in positive, negative, neutral and mixed (positive and negative) ways. The first study (Study 1a) showed that the valence of autobiographical reasoning found in young adults' life story narratives predicted psychological functioning. Young adults who made negative self-event connections in life story narratives experienced poorer psychological functioning (measured in terms of psychological distress and psychological well-being) than young adults who made little or no negative self-event connections. Conversely, young adults who made more positive self-event connections experienced comparatively better psychological functioning than those who made fewer positive selfevent connections. The relationship between positive self-event connections and positive psychological functioning was most salient in the context of narratives about negative events from the life story. Study 1a also showed that for young adults who tended to make higher numbers of positive self-event connections, endorsing negative events as central to the life story was not associated with poor psychological functioning, whereas it was for young adults who made fewer positive connections. The second study (Study 1b) presented a methodology for examining the relationship between autobiographical reasoning valence and psychological functioning over time. Although the small sample size in Study $1 \mathrm{~b}$ prevented firm conclusions being made, findings showed that young 
adults' tendency to make negative, but not positive, self-event connections remained stable over time. The preliminary findings from Study $1 \mathrm{~b}$ also showed that positive and negative self-event connections in life story narratives were not associated with changes in psychological functioning over time. The third study (Study 2) found that young adults' tendency to reason about the self in positive and negative ways was associated with a number of cognitive response styles (explanatory style, rumination and use of cognitive reappraisal strategies). The results of Study 2 also highlight important ways that cognitive response factors, and young adults' assessments of meaning in their lives, may interact with autobiographical reasoning valence to predict psychological functioning. The fourth study (Study 3) aimed to investigate relationships between the phenomenology of life story memories and the amount, and valence, of autobiographical reasoning in narratives of these events. Findings showed few associations between autobiographical reasoning and autobiographical memory phenomenology. Possible reasons for the absence of these relationships are discussed. Wider implications and theoretical explanations for the findings reported in this thesis are discussed in terms of models of coping and Relational Frame Theory (RFT; Hayes, Barnes-Holmes, \& Roche, 2001). 


\section{Acknowledgements}

There are so many people to thank for their help and support with this thesis. First, to my supervisor Karen Salmon, thank you for your support and encouragement throughout this process. Thank you for sharing your knowledge with me, for your valuable feedback and for constantly encouraging me to think critically about the issues discussed in this thesis. Thank you also to my secondary supervisor Paul Jose who provided valuable input and feedback at much needed times. I would also like to thank Danica McGovern, Shelley Kennedy and Rebecca Burson, who helped with reliability coding. Coding the life story narratives was not always an easy and straight-forward task and I appreciate the time and dedication you gave to making sure the narratives were accurately represented in the data. I would also like to thank the young adults who participated in the studies, for sharing parts of their life story with me so that I could undertake this research. I also owe thanks to the Faculty of Science for their financial support that allowed me to attend the conference 'Clinical perspectives on autobiographical memory' to present data from this thesis.

On a personal level, I would very much like to thank the tremendous amount of support and encouragement that was given to me by my support network. Sam, you have shared this journey with me from the beginning and have been such a 'rock' throughout the process. Thank you for your love and never-ending encouragement. I owe a tremendous amount of thanks to Danica McGovern for the amazing 'peer supervision' sessions. I only hope that I can help you with your own $\mathrm{PhD}$ journey as much as you have helped me. Thanks to Mum and Dad for supporting me through all the ups and downs, for your love and encouragement, and unconditional support (both emotional and financial!). Thanks also to my sister Julia and the wider Banks family for your encouragement and words of wisdom at key times. To my Wellington 'family', and work 'family' at the Ministry of Education, thank you for your endless support and for encouraging me to have confidence in my ability to complete this thesis.

Lastly, but by no means insignificantly, I would like to thank my Nana, Muriel Lee, for sharing your life story with me and inspiring me to research the life story from a psychological perspective. 


\section{Table of Contents}

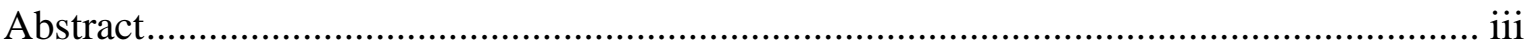

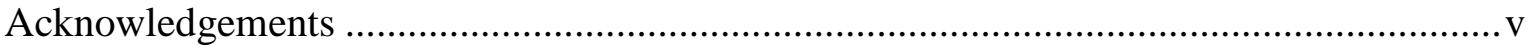

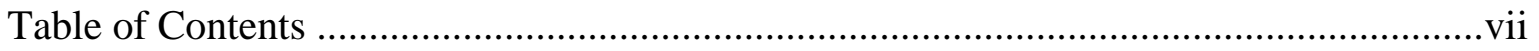

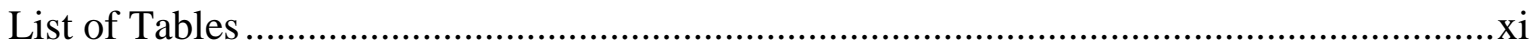

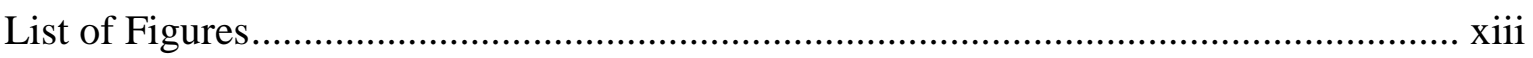

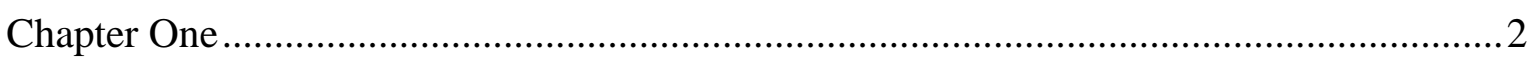

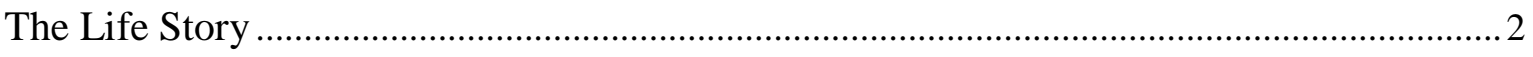

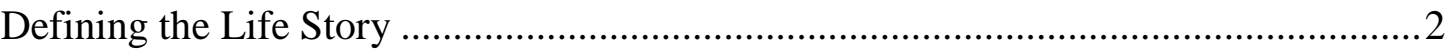

Autobiographical Memory and the Life Story- Developmental Considerations............3

The Role of Autobiographical Reasoning in the Development of the Life Story ..........5

McAdams’ Life Story Model of Identity: Concepts and Considerations........................ 10

Identity Construction and Life Story Development in Young Adulthood ..................... 11

Influence of Social Interaction on the Development of the Life Story ......................... 16

Influence of Culture on the Development of the Life Story ........................................ 17

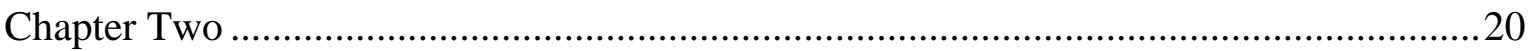

Autobiographical Reasoning, Life Story Development and Psychological Functioning .... 20

The identity function of autobiographical reasoning - links to psychological

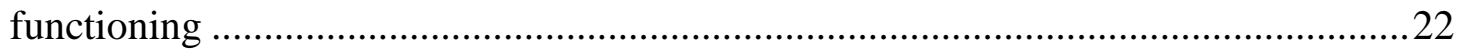

Adaptive patterns of meaning making - links to psychological functioning................26

Centrality of life story events and psychological functioning....................................31

Justification for Current studies ........................................................................... 32

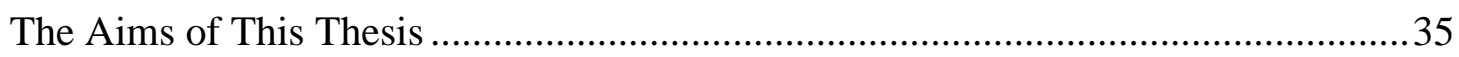

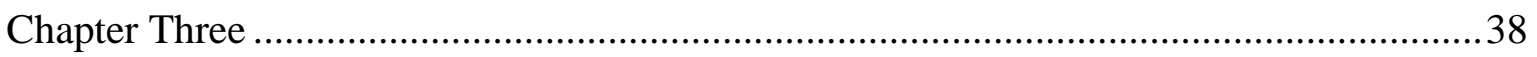

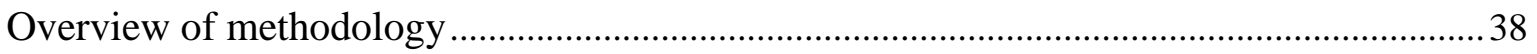

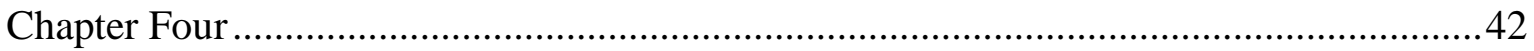

Study 1a: The relationship between autobiographical reasoning processes and content,

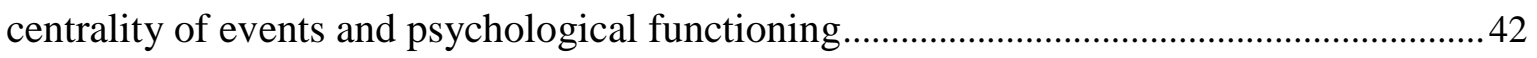

Autobiographical reasoning and identity development ......................................................... 43

Autobiographical reasoning and psychological functioning................................................. 44

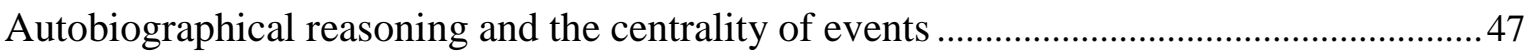

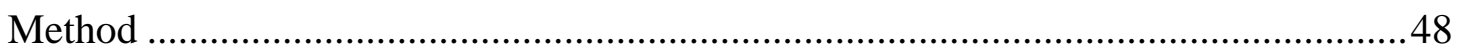

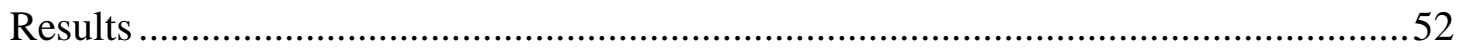


Discussion 62

Chapter Five

Study $1 \mathrm{~b}$ : Does the valence of autobiographical reasoning found in life story narratives contribute to changes in psychological functioning over time? A pilot analysis . .70

Method .73

Results .75

Discussion .76

Chapter Six

Study 2: An investigation into the relationships between autobiographical reasoning, cognitive response styles, meaning in life and psychological functioning 82

Outline of cognitive response styles. .83

Similarities between cognitive response styles and autobiographical reasoning..... .85

Part 1a: Relationship between positive/negative self-event connections and cognitive response styles

Part 1b: Relationships between positive/negative self-event connections, cognitive response styles and psychological distress

Introducing the concept of meaning in life .90

Relationship between autobiographical reasoning valence and meaning in life. 91

Part 2a: Relationship between autobiographical reasoning valence and meaning in life

Part 2b: Relationships between autobiographical reasoning valence, meaning in life and psychological well-being.

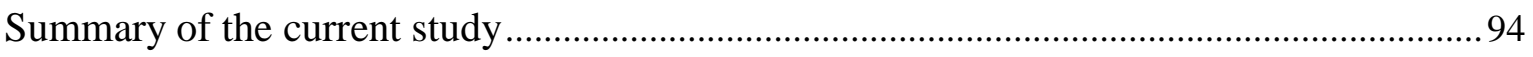

Method .95

Results .99

Discussion

Chapter Seven

Study 3: An investigation into the relationships between autobiographical reasoning, psychological functioning and the visual recall perspective and subjective distance of life story memories

Visual perspective

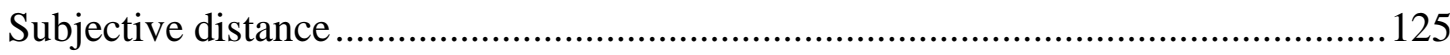

Visual perspective and psychological distance of life story events....

Relationships among visual perspective, subjective distance and autobiographical reasoning found in life story narratives 
Relationships among visual perspective and psychological functioning ..................... 128

The relationship between subjective distance and psychological functioning ............ 132

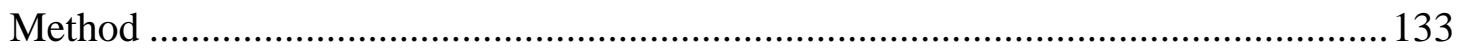

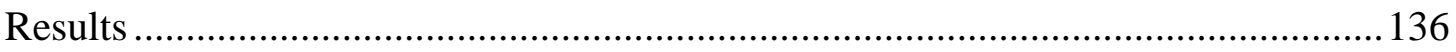

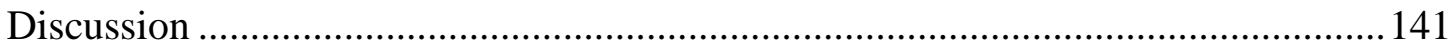

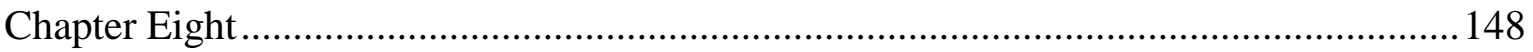

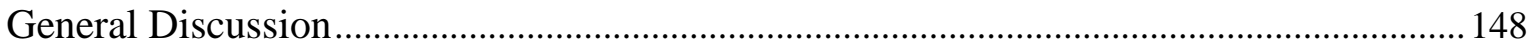

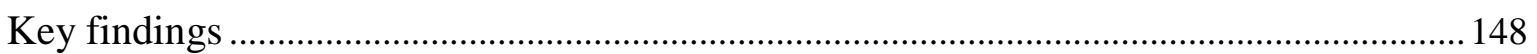

The development of the life story and positive psychological functioning ............... 150

The development of the life story and poor psychological functioning...................... 154

Can Relational Frame Theory (RFT) explain the relationship between negative selfevent connections and psychological distress? ........................................................ 158

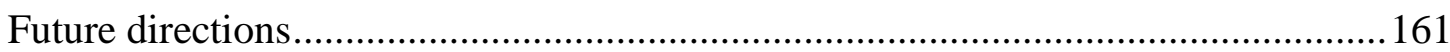

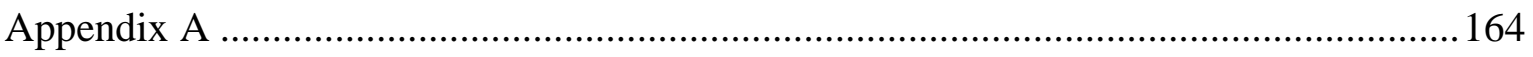

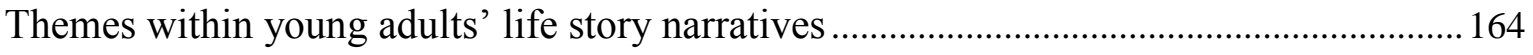

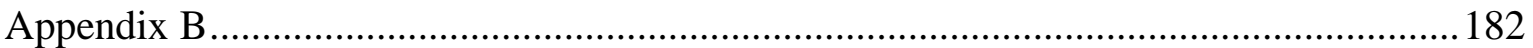

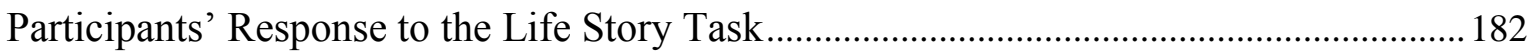

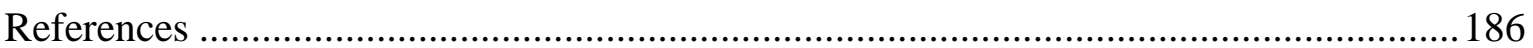




\section{List of Tables}

Table 4.1 Means and percentages for narrative variables and centrality ratings across memory type

Table 4.2 Correlations amongst narrative variables and psychopathology/well-being scales

Table 4.3 Stepwise regression of narrative variables in the prediction of DASS scores .55

Table 4.4 Stepwise regression of narrative variables in the prediction of RYFF scores..56

Table 4.5 Correlations between centrality ratings for high and low points, narrative variables and psychopathology/well-being scales

Table 5.1 Differences in participant characteristics and autobiographical reasoning measures at Time 1 and Time 2 (one year later)

Table 6.1 Means, standard deviations and ranges for self-event connections across memory type

Table 6.2 Correlations amongst narrative and cognitive variables

Table 6.3 Correlations amongst narrative variables and psychopathology/well-being scales

Table 7.1 Means, standard deviations and ranges for visual perspective and subjective distance ratings for each memory type

Table 7.2 Correlations between the number of negative self-event connections made in narratives of low points and use of the observer recall perspective

Table 8.1 Correlations between positive and negative self-event connections and psychological functioning across three studies

Table B1 Participants' experience of the life story narrative task

Table B2 Correlations amongst participants' subjective assessments of the enjoyment and impact of the autobiographical narrative task, and positive and negative self-event connections 


\section{List of Figures}

Figure 4.1. Moderation of the relationships between number of self-event connections, sophistication of meaning and DASS scores by proportion of negative self-event connections in narratives of low points

Figure 4.2. Moderation of the relationships between number of self-event connections, sophistication of meaning and RYFF scores by proportion of positive self-event connections in narratives of low points

Figure 4.3. Moderation of the relationship between DASS scores, RYFF scores and centrality of event ratings for low points by proportion of positive self-event connections in narratives of low points

Figure 6.1. Moderation of relationship between number of negative self-event connections found in narratives of low and turning points and psychological distress, by negative explanatory style

Figure 6.2. Mediation of the relationship between the number of negative self-event connections in narratives of low and turning points and psychological distress, by rumination.

Figure 6.3. Moderation of relationship between number of positive self-event connections found in narratives of high, low and turning points and satisfaction with life, by meaning in life

Figure 6.4. Mediation of the relationship between negative self-event connections in narratives of high, low and turning points and Satisfaction with Life ratings by meaning in life ratings

Figure 8.1. Hypothesised relationship between negative self-event connections, centrality of negative events and psychological distress 


\title{
Chapter One
}

\author{
The Life Story
}

\begin{abstract}
"Now that I have put pen to paper and written my life story I am pleased it is all out in the open. I could never bring myself to discuss my story, so consequently it festered inside me. But that has now changed, and thank goodness"
\end{abstract}

Muriel Lee, 92 years of age

There is something inherently powerful about the life story. The above quote from the life story of my paternal grandmother conveys just some of the influence that her life story had on her sense of well-being, and the subsequent relief she experienced upon writing her story and sharing it with others. This thesis is centred on the theoretical notion that our life stories are intricately associated with our perceptions and beliefs about who we are as individuals (McAdams, 1988; 2001; McLean, Pasupathi, \& Pals, 2007). Within this broad theoretical context, the central aim of this thesis was to address the question of whether, and how, the development of the life story relates to psychological functioning. These questions were examined in the developmental period of young adulthood, a theoretically significant time for the construction of the life story and identity. The purpose of this Chapter is to discuss the theoretical notion that the life story comprises narrative identity (McAdams, 1988; 1993) and explore three related questions 1) what is the life story, 2) how is the life story formed, and 3) what role does the life story play in our lives? This body of conceptual, theoretical and empirical work provides the necessary contextual and background information for the central question of this thesis, namely, whether and how the life story relates to psychological functioning in young adulthood.

\section{Defining the Life Story}

From a theoretical perspective, the life story is constructed from autobiographical memory, broadly defined as memory for our own experiences (Conway, Singer, \& Tagini, 2004; Loftus \& Fathi, 1985; Nelson \& Fivush, 2004). As individuals, we experience a large number of events both in our everyday lives and over our lifetime (Davis \& Burns, 1999; Heady \& Wearing, 1989). Yet only a portion of autobiographical memories for these experiences are included in the life story (Bluck \& Habermas, 2001). Thus while 
autobiographical memories provide the raw material from which the life story is based, the construction of the life story is considered to be more complex than the simple recollection and recounting of autobiographical memories.

According to theorists such as McAdams (1988; 2001) and Pillemer (1998; 2001), the events that comprise the life story are the most personally relevant experiences of an individuals' life. These highly personally relevant events have been defined and operationalised in a number of ways. McAdams (1988) defines these events as "nuclear episodes', "autobiographical events which ... tend to be understood and felt as uniquely ours" (p. 63). Pillemer (2001) alternatively suggests that the life story is likely to contain memories for 'momentous events', defined as "distinctive, circumscribed, highly emotional and influential episodes" in a person's life (p. 123). Singer and Moffitt (19911992) suggest that life story events are those which are 'self-defining', events which cause strong emotions, have been thought about many times, feel important and help individuals understand who they are.

Although worded slightly differently, these definitions of life story events all imply that the events which comprise the life story gain personal relevance because they convey important information about who a person is, or explain why they are the person that they are today. Consistent with this interpretation, Bluck and Habermas (2000) suggest that events included in the life story often provide an explanation for changes or developments in the individual. Similarly, Pillemer $(1998 ; 2001)$ argues that memories for momentous events often contain within them life lessons that continue with the individual long past the initial experience. Pillemer (1998; 2001) further states that momentous events also encompass experiences that caused a change in individuals' long-term goals or life-path (termed 'originating' and 'turning point' events) or solidify an individuals' belief system (termed 'anchoring' events). These descriptions of life story events suggest that the way individuals process and reflect on their life events plays an important role in determining their personal relevance. Having established a working definition of the life story, I will now turn to the question of how the life story develops. Gaining this developmental perspective provides an important theoretical context for considering claims of whether, and by what means, the life story becomes narrative identity.

\section{Autobiographical Memory and the Life Story-Developmental Considerations}

According to key developmental theorists, autobiographical memory develops from a very young age in the context of social interactions and conversations with adults (Fivush, 2007; Fivush \& Nelson, 2006; Nelson \& Fivush, 2004). Children between the 
ages of 18 months and three years become increasingly involved in parent-child conversations about past events (Haden, Ornstein, Rudek, \& Cameron, 2009). Key proponents of the social-interaction theory of autobiographical memory development suggest that through exposure to, and engagement in these conversations, children learn to distinguish between past and present events and discover how to construct a narrative, or story, about a personally experienced event (Nelson, 2003).

Parents differ in the extent to which they encourage children to contribute and elaborate on information within conversations, and such differences in conversation style have been associated with qualitative differences in children's memory over time. In one study, Reese, Haden, and Fivush (1993) recorded conversations between mothers and their children at four time points (when the child was 40, 46, 58, and 70 months of age). Each conversation was coded for parent and child factors. Parent factors included elaborations (utterances which introduced new information or directed the conversation to new aspects of the experience) and repetitions (utterances which repeated the content of a previous statement). Child factors included memory responses (utterances that introduced new information, directed the conversation to new aspects of the experience or asked the parent for more information), repetitions and evaluations (utterances expressing agreement/disagreement with a comment made by the parent). Some mothers, described as 'highly elaborative', made more elaborative statements than repetitive statements, while other mothers, described as 'low elaborative', made an equal number of elaborative and repetitive statements. Correlational findings showed that children of highly elaborative mothers recalled more information (i.e. made more memory responses) than children of low-elaborative mothers. Longitudinally, children exposed to high levels of parental elaboration during early developmental stages (i.e. when the child was 40 months of age) provided more elaborative personal narratives in later years. These findings suggest that initially, children of highly elaborative mothers make more memory contributions in conversation with parents at an early age and, in later years, independently construct more elaborate memory narratives (see also Fivush, 2011; Fivush, Reese, \& Haden, 2006).

From a theoretical perspective, early parent-child conversations contribute not only to the development of autobiographical memory, but also to the development of the self or 'self-concept'. In highlighting the crucial role of language in the development of the self and autobiographical memory, Nelson (2003) notes that by discussing past events with children, parents communicate the personal importance and/or meaning of the child's experiences to them. That is, engaging in conversations about past events provides the 
child with a sense that the event is of personal importance to them. In addition, discussing the child's internal experiences (e.g. thoughts, beliefs and emotions) and their actions or behaviours provides the child with a sense of self, that in turn is embedded within the memory for the past experience (Fivush \& Nelson, 2006).

In particular, conversations about past events that elicited negative emotions in the child may have particularly strong connection to self-concept development (Fivush, Berlin, McDermott Sales, Mennuti-Washburn, \& Cassidy, 2003). Bird and Reese (2006) recorded parent-child conversations about emotional experiences (e.g. experiences in which the child felt angry, sad, scared, and happy). Conversations were coded for certain ways of discussing emotions, including whether the emotion was explained, confirmed and/or resolved. The children (between 63 and 81 months of age) also rated the extent to which various self-descriptors applied to them along nine dimensions (e.g. achievement - strives to perform highly, social closeness - enjoys being around others). Children who demonstrated greater consistency in their self-views (i.e. their self-views were present across contexts) were more likely to be exposed to parent-child conversations where negative emotions were explained. This finding was interpreted as evidence that highlighting the personal meaning of events through conversational narratives helps children to understand their emotional experiences and hence understand themselves better. The findings also highlight that from an early age, knowledge about who we are and what is important to us as a person, becomes closely associated with our memory for past experiences.

In summary, autobiographical memory develops from an early age in the context of conversations about the past. Children of parents who tend to be more elaborative in recounting past events in these conversations tend to produce more elaborative memory narratives when recounting past events on their own. Parent-child conversations about the past, and in particular, conversations about negative emotional experiences may help children understand the personal significance of their experiences. While this developmental perspective provides an important context for understanding the emergence of autobiographical memory, at what point does the ability to recall autobiographical memories develop into the ability to construct a life story? This is an important question that will now be addressed.

\section{The Role of Autobiographical Reasoning in the Development of the Life Story}

Although autobiographical memory and story-telling capacities may start to develop in childhood, from a theoretical perspective, the ability to construct a life story 
does not emerge until adolescence or young adulthood (Habermas, 2010; McAdams, 1988). Habermas and Bluck (2000) suggest that the ability to construct the life story coincides with developmental changes that take place during this lifetime period. The first change is a social and cultural drive to conceptualise oneself in biographical terms, that is, to think about who one is as an individual and communicate this to others. The second factor is the development of a set of social-cognitive competencies that enable individuals to engage in a unique form of reflection termed 'autobiographical reasoning'.

Autobiographical reasoning is defined as a process of reflection in which individuals draw connections between past experiences and enduring and current characteristics of the self (Habermas \& Bluck, 2000). Although the term 'self' is not explicitly defined in Habermas and Bluck's (2000) definition of autobiographical reasoning, it is used synonymously with the terms 'self-characteristics' and 'selfdefinition'. Thus the process of autobiographical reasoning from a theoretical perspective involves making connections between past events and descriptive and/or evaluative information about the self (see Singer \& Bluck, 2001; McLean et al., 2007).

In addition to the development of autobiographical reasoning capabilities Habermas and Bluck (2000) propose that the emergence of the life story also coincides with the development of four social-cognitive capabilities. These include the ability to order past events in a temporal manner (temporal coherence), the ability to think about the self in abstract terms (i.e. as embodying certain personality traits) and account for changes or developments in the self over time (causal coherence, of which autobiographical reasoning is a part), the ability to summarise and interpret themes within stories and apply these to one's own life (thematic coherence), and having an awareness of cultural norms regarding the major milestones and events one is expected to experience during the life course (Habermas \& Bluck, 2000).

Habermas and colleagues (Bluck \& Habermas, 2000; Habermas \& de Silveira, 2008) have tracked these social-cognitive changes in life stories across the developmental period from childhood to young adulthood. Habermas and de Silveira (2008) asked 8, 12, 16 and 20 year olds to orally narrate their entire life story and coded these narratives for temporal, causal and thematic coherence. Findings showed an increase in all three coherence measures during adolescence. Compared with children, adolescents made more references to life phases and correct dates and times, statements indicating causal links between events and between events and the self, biographical arguments relating to their developmental context or development over time, generalised statements about themes or 
consistencies evident in their experiences, and complex cognitive processes including statements indicating an understanding or opinion of their experiences than did children in the younger age groups.

In addition to these indicators of life story development, there is evidence that autobiographical reasoning increases across the developmental periods of adolescence and young adulthood. Before examining empirical support for this claim, it is useful to consider how autobiographical reasoning is conceptualised and operationalised in measurable terms.

Autobiographical reasoning has been conceptualised in two main ways; as selfevent connections and sophistication of meaning. Self-event connections, first described by Pasupathi, Mansour, and Brubaker (2007), are defined as explicit statements that describe a link between a personal experience and an aspect of the self. Aspects of the self referenced in self-event connections frequently include personal traits and dispositions, values, attitudes and beliefs about the world and the development of personal strength/ growth (McLean \& Fournier, 2008; Pasupathi \& Mansour, 2006). The following is an example of a narrative containing self-event connections. This excerpt is from the life story of a female young adult who was 20 years of age at the time the narrative was constructed.

"I was 18 [years old] and caught my long term boyfriend cheating ... I think this is an important event in my life because I discovered how well I could cope with a bad situation. I have learnt to accept and let go of bad things that happen"

This example highlights two salient self-event connections. First, the young adult describes how the experience of catching her boyfriend cheating lead her to realise how well she is able to cope with a difficult situation. Second, she attributes the development of a current trait in herself (the ability to accept and let go of bad things) to this particular experience. From a theoretical perspective, this self-discovery and insight into her selfdevelopment occurred during the young adult's reflection and thinking about the event after it had happened. This process, and the links she formed between event and self, increased the likelihood that the experience would be included in her life story (Pasupathi et al., 2007).

The second conceptualisation of autobiographical reasoning, sophistication of meaning, was first described by McLean and Thorne (2003). Sophistication of meaning is characterised as the level of insight or complexity of knowledge - particularly about the 
self - that is attained as a result of reflecting on one's past and self. McLean and Thorne (2003) proposed that gaining broader insights into the self through reflecting on past experiences represents a deeper level of reflection and post-event processing than learning specific lessons. Lesson-learning no doubt serves a very useful function in individuals' lives. That is, a lesson learnt from a specific experience can guide future behaviour in order to avoid aversive situations or maximise positive outcomes in the future. The following example from the life story of a young male adult, 22 years of age, highlights the lesson-learning process:

"I was twenty one when I was out one night and was caught drink driving for the second time ... I drove straight into a checkpoint ...I alone was in the car and made the choice to drive ... This is important because if I am caught again I will go to jail, it's that simple ...I have actively toned down my drinking habits and will never drink drive again"

Although not explicitly framed as a life lesson by the young adult himself, his changes in behaviour (consuming less alcohol and choosing not to drive under the influence of alcohol again) are clearly linked to this experience of being caught. These decisions and subsequent behaviour change will conceivably be a positive factor in his life as it will allow him to avoid negative consequences (going to jail, having an accident) in the future.

In contrast to lesson learning, gaining insight from a past experience involves a shift in one's thinking or understanding that extends beyond specific behaviours or situations. McLean and Thorne (2003) suggest that gaining insight often involves a change in individuals' understanding and beliefs about themselves, others and/or the world. Gaining insight is illustrated in the following example:

"When I was 17 years old I got accepted into university ... It was exciting as I felt like I was starting something new and there were many opportunities for me. It also gave me joy that I knew I had got in and passed [secondary school qualification] ... This event showed me that I am a hard working person and know when to put the effort in ... it makes me feel worthwhile and like I have accomplished something"

In this example, the young adult realised that she possesses two attributes, that is, she is hard-working and has the ability to apply effort when needed. Although she links these two attributes to this one experience, they represent general qualities that the young adult has that can be applied in numerous areas of her life. As gaining insight involves 
reflecting on broader, more abstract self-characteristics as opposed to the focus on concrete behavioural patterns in lesson learning, gaining insight is considered to reflect greater complexity, or sophistication, of reflection (McLean and Thorne 2003; Thorne, McLean, \& Lawrence, 2004).

There are a number of key similarities and differences between the two conceptualisations of autobiographical reasoning, self-event connections and sophistication of meaning. Self-event connections and sophistication of meaning both refer to the extent to which individuals derive information about the self from their life events; however, they do so in slightly different ways. Self-event connections capture any explicit links a narrator has made between event and self, and therefore provide a basic measure of the extent to which autobiographical reasoning has occurred. Sophistication of meaning extends the measurement of this basic process by capturing the degree to which this knowledge describes abstract characteristics of the self such as 'hard-working' (high level of sophistication) or concrete behavioural lessons 'I will not drink and drive' (lower level of sophistication). Having outlined how autobiographical reasoning is operationalised, I now return to the issue of developing autobiographical reasoning capabilities in adolescence and young adulthood.

There is some evidence that autobiographical reasoning increases across the adolescent years. McLean and Breen (2009) asked adolescents, 14 to 18 years of age, to write about a turning point from their life story, and coded each narrative for sophistication of meaning. Overall, sophistication of meaning ratings increased across the age groups, with the most marked increase between the ages of 16 and 17 years. These findings show that compared with younger adolescents (14 years of age), older adolescents (18 years of age) were more likely to report insights gained into the self in the context of narratives about turning points from the life story. In a related study, Pasupathi and Mansour (2006) asked adults between 18 to 86 years of age to narrate a turning point and a crisis they had experienced in their life. Each narrative was coded according to whether self-event connections were present or not. Age was significantly associated with the presence of self-event connections in narratives of crises but not turning points. Within narratives of crises, the likelihood of eliciting a narrative containing self-event connections increased during young adulthood. Taken together, these findings suggest that the presence of autobiographical reasoning in narratives of major life events increases over the adolescent and young adulthood periods. 
Earlier in this Chapter, I suggested that the events which comprise the life story hold a high level of personal significance for the individual. The concept of autobiographical reasoning provides a useful way of assessing the personal significance of one's life events. By definition, autobiographical reasoning is a process of reflection in which individuals draw connections between past experiences and enduring and current characteristics of the self (Habermas \& Bluck, 2000). Thus by drawing these connections, individuals intertwine elements of themselves into the memory for the past experience, which in turn, gives the experience personal significance. Consistent with this claim, narratives of events from the life story contain more evidence of autobiographical reasoning than do narratives of autobiographical memories not included in the life story (Grysman \& Hudson, 2010). Thus from a theoretical perspective, the life story comprises events which have personal significance, which in turn is garnered through reflecting on the broader meaning of the event for the self (Habermas \& Bluck, 2000).

In summary, the development of autobiographical reasoning and the subsequent emergence of the life story is observed during the adolescent and young adulthood years. The foundations for the development of these capabilities can be tracked right back to early childhood, and the development of autobiographical memory in the context of parent-child conversations about the past. Key theorists (see Fivush, Hazzard, McDermott Sales, Sarfati, \& Brown, 2003; McLean, Pasupathi, \& Pals, 2007; McLean, Breen, \& Fournier, 2010) have suggested that parental elaboration may be a precursor to autobiographical reasoning as this style of recalling past experiences involves greater levels of reflection than non-elaborative recall. In light of this developmental context, I turn to a discussion of McAdams' Life Story Model of Identity (McAdams, 1988; 1993; 2001).

\section{McAdams' Life Story Model of Identity: Concepts and Considerations}

Life story theorist Dan McAdams suggests that the life story comprises, or is, an individual's narrative identity (McAdams, 1988; 1993; 2001). Although this thesis does not explicitly test the central theoretical claim of McAdams' model, the notion that identity is the life story provides the broad theoretical framework from which earlier psychological research on the life story, and this thesis is based (Angus, Levitt, \& Hardtke, 1999; Brooks \& Dallos, 2009; Hill \& Dallos, 2012; McLean, 2005; 2008; McLean \& Breen, 2009).

The key theoretical premise of McAdams' Life Story Model of Identity, that narrative identity is the life story, was formulated in relation to the early developmental theory of Erikson (1968). Erikson (1968) asserted that the major developmental task 
facing young adults as they transition from adolescence to adulthood is the development and strengthening of identity. Although the life story approach to identity development taken by McAdams is based on this core theoretical principle, the way in which identity is defined by the Eriksonian and life story models is considerably different. According to Erikson (1968), human development passes through key stages, with each stage posing new and difficult challenges that need to be achieved. The primary achievement of the adolescence period according to this perspective involves answering questions of identity, that is, "who am I" and "How do I fit into the adult world". Answering these questions involves careful consideration of the various roles, ideological and religious beliefs, attitudes and personal characteristics one can and wants to hold (identity exploration), and making a commitment in terms of self-definition and action that satisfies these characteristics and virtues (Waterman, 1982). Thus identity, from this early (Eriksonian) perspective, comprises the consortium of individuals' thoughts, beliefs, values and goals held in relation to various life domains, and the actions and behaviours taken by the individual in accordance with these. Erikson's theory of identity has been difficult to validate empirically, however, and no evidence has been found for the presence of set developmental 'stages'. Rather, indicators of identity achievement have been shown to increase across adulthood (Whitbourne, Sneed, \& Sayer, 2009).

\section{Identity Construction and Life Story Development in Young Adulthood}

McAdams suggests that function of the life story is identical to that of Erikson's (1968) identity theory, namely, to answer the question of "who am I?". Similarly, both Erikson (1968) and McAdams (1988; 1993) agree that identity develops during the adolescent and young adulthood years. The point of difference between Erikson's (1968) and McAdams' (1988; 1993) models is the proposed means by which identity develops. Unlike Erikson, McAdams $(1988$; 1993) suggests that identity is developed through the life story. McAdams $(1988 ; 1993 ; 2011)$ further states that the life story is an internalised narrative that individuals construct in order to 'make sense' of, or understand, who they are and how they developed into the person they are today.

McAdams and colleagues suggest that narrative identity is one component of a broader framework of human personality ${ }^{1}$ (McAdams, 1995; 1996; McAdams \& Pals, 2006). According to this framework, personality can be assessed at three hierarchical

\footnotetext{
1 The conceptualisation of narrative identity as a component of personality is based on the notion that the goal for personality psychology is to provide a framework for describing and understanding people (McAdams \& Pals, 2006).
} 
levels, with each level providing increasingly specific and detailed information about the individual (McAdams, 1995; 1996; 2001). The first level in the hierarchy includes trait descriptions akin to those found in traditional measures of personality (e.g. 5-factor models, see Digman, 1990). According to McAdams, these traits provide broad descriptive categories that can be applied to individuals, but lack flexibility in terms of describing individuals' behaviour across settings and contexts (see Wright \& Mischel, 1987). For example, an individual may be described as 'extroverted', which denotes a certain pattern of behaviour, but does not guarantee that an individual will behave in a certain way in a range of settings. These descriptions can also be applied to many other individuals and thus are not unique to the person to which it is applied. The second level of the hierarchy includes constructs such as motives, goals, values and identifiable patterns of behaviour that are concerned with what individuals want from their lives. These 'personal concerns' can, and do, show temporal and contextual flexibility but again can be shared by a number of people and thus are not entirely unique to the individual.

The third level of McAdams' hierarchy comprises narrative identity, an internalised narrative, "inner story", that describes the most important experiences in an individual's past and explains their development as a person within the context of this personal past (Bauer, McAdams, \& Pals, 2008; McAdams, 1996; 2001; 2011). Unlike the information contained within the first two levels, an individuals' life story, including the actual life events experienced by the individual and the manner in which they are recalled and reconstructed, is entirely unique to the individual. The term 'narrative identity' is used specifically in this model to illustrate the important theoretical role of narrative as a vehicle for making sense of, and understanding ourselves and our experiences (McAdams, 2011).

Despite its widespread acceptance, there are a number of unresolved issues in McAdams' Life Story Model of Identity. One of the key issues is clarity regarding the definition of the terms; 'narrative identity' and 'life story'. Of particular concern, the definitions of the terms narrative identity and the life story in McAdams' model are inherently circular. That is, each construct is defined only in terms of its counterpart - the life story is narrative identity and narrative identity is the life story. Sound theories should have well-defined terms that are explicit and operationalised (Prochaska, Wright, \& Velicer, 2008). McAdams' model does not offer any additional explanation or definition of narrative identity outside the notion that narrative identity $i s$ this internalised narrative. 
Theoretical suggestions regarding the definition of identity that have been made outside of McAdams' model may be useful when considering the concept of narrative identity.

Vignoles, Schwartz and Luckx (2011) acknowledge that although the notion of identity is a popular construct within psychology, it has been notoriously difficult to define. These authors note that although precise definitions vary, identity tends to be defined in one of three ways; as individual, relational and/or collective identity (Sedikides \& Brewer, 2001). Where individual, or personal, identity is defined as the self-definitions that individuals employ to describe themselves, relational identity is defined as individuals' relationships in terms of others (e.g. mother, teacher) and collective identity is defined as individuals' identification with groups and social classifications (Vignoles et al., 2011). While all of these definitions of identity are related to how individuals answer the central question of "who am I?" the focus of personal identity is on internal characteristics, while the focus of relational and collective identity focuses on relationships that are external to the individual. Elements of the life story as described by McAdams may fit within each of these types of identity. If the development of the life story is achieved through autobiographical reasoning as discussed above, however, then the life story from a theoretical perspective is closely aligned with the notion of individual or personal identity, the self-definitions that individuals employ to describe themselves.

McAdams agrees that the life story serves a self-understanding function individuals construct a life story in order to understand who they are. It is not clear within his model, however, whether narrative identity is the self-understandings that are achieved through the process of constructing a life story or whether narrative identity refers to a self that encompasses much more than the beliefs and understandings a person has about who they are (see Ward, 2012). Further adding to this lack of clarity, the terms 'self' and 'identity' or 'narrative identity', are used somewhat interchangeably in the life story literature. Perhaps the clearest explanation of the relationship between the self, selfunderstanding, and the life story is provided by proponents of McAdams' model, McLean, Pasupathi, and Pals (2007). These authors suggest that the self comprises both the life story and the self-concept, where the self-concept is defined as "conscious beliefs about the self that are descriptive or evaluative" (p. 263). McLean et al. (2007) suggest that the self-concept and life story are inherently linked, in that some elements of the self-concept are reflected in the life stories through the process of autobiographical reasoning. In light of this theoretical support, it seems reasonable to expect that narrative identity comprises, 
at least in part, the self-understandings that an individual expresses through the construction of the life story.

An additional issue that theories of identity, narrative or otherwise, have had to address is whether individuals have one single, or multiple identities. Vignoles et al. (2011) suggest that identity is multifaceted in the sense that individuals can describe themselves in many different ways, for example, as a daughter, an extrovert, and academically able. In a similar manner, narrative identity is conceptualised as multifaceted in the sense that individuals do not simply have one life story. That is, the life story may comprise any number of experiences an individual has had over their lifetime, the life story is continually updated as individuals gain new experiences, and the life story that is shared with others may depend on the social context in which it is told (Habermas \& Bluck, 2000; McAdams, 1988; 2001; Pasupathi, 2001).

In light of the limitations of McAdams' model, particularly the definition of key terms, the notion that narrative identity is the life story which emerges during the adolescent and young adult years, has been notoriously difficult to test. Support for this claim has instead relied on relationships to more indirect assessments of identity and developmental changes in the purpose of autobiographical remembering. For example, Webster and McCall (1999) found that younger adults were more likely to report that they reminisce for the purpose of seeking out or clarifying information about who they are (e.g. when I reminisce it is to try to understand myself better) than were older adults. Bluck and Alea (2009) found that relative to older adults, younger adults were more likely to report thinking and talking about their past for the purpose of creating 'self-continuity'. That is, young adults were more likely to think about the past with the aim of gaining a sense of stability over time or explaining changes in the self over time. These studies did not examine reminiscence in the context of the life story, nor did they explicitly assess identity development. Instead, an assumption was made that identity development is a greater developmental concern for the young adults in their studies, and that these findings provide some evidence for this.

Some support for the notion that autobiographical reasoning is associated with the development of identity in adolescence and young adulthood has also been derived from research investigating the self-reported use of sharing key life story events. McLean (2005) asked young adults to write narratives of three self-defining memories and for each memory, to state whether they had told the memory to others and if so, what the purpose was for telling the memory to others. Each narrative was then coded for sophistication of 
meaning, the extent to which participants learnt lessons or gained an insight into the self as a result of the event. Memories were most commonly disclosed to others for the purpose of self-explanation, that is, to explain oneself to someone and for the purpose of entertaining others. Statements depicting insight into the self and lessons learnt were more commonly found in narratives of autobiographical memories that were shared with others for the purposes of self-explanation than in narratives of memories shared for the purpose of entertainment (McLean, 2005). One interpretation of these findings is that disclosing memories for the purposes of self-explanation may help the individual discover and strengthen their views about who they are, which is a core feature of identity development as proposed by the life story approach. One limitation of this work, however, is that participants' views about the purpose for telling the memory was retrospective and thus may have been influenced by the experience and outcome of telling the memory to others. That is, participants who gained insights into the self from telling the memory to others may be more likely to assume that they had this self-explanatory goal leading up to the disclosure.

Attempts have also been made to link the Eriksonian and life story perspectives on identity development. McLean and Pratt (2006) administered a measure of identity status to a sample of adolescents over a six year period as they transitioned to young adulthood (mean age at time $1=17$ years, time $2=19$ years, time $3=23$ years). The measure of identity status contained four states of identity development resulting from the interrelation between identity exploration and commitment. These four states included 'identity achievement' (individual has both explored and committed to an identity), moratorium (exploration has occurred but no commitment), 'foreclosure' (individual has committed to an identity without exploring their identity), and 'diffusion' (no evidence of exploration or commitment). At the last time point, the young adults were also asked to narrate a turning point from their life story. Results showed that young adults who, at the age of 23 , made less sophisticated meaning in narratives of turning points were more likely to be in a state of foreclosure (where they demonstrated commitment to an identity in the absence of exploration) and diffusion (where they demonstrated no exploration or commitment to identity) than young adults who made more sophisticated meaning. These findings were taken to suggest that while autobiographical reasoning may not be required to successfully achieve identity, the absence of autobiographical reasoning may reflect sub-optimal identity development. 
In summary, the central premise of McAdams' Life Story Model of Identity is that identity is the life story. In this Chapter, I have suggested that the life story is comprised of autobiographical memories that are considered highly personally relevant to the individual. One of the key ways through which autobiographical memories gain personal significance is through autobiographical reasoning, a process of reflection in which individuals construct links between past life experiences and descriptive/evaluative statements about their current self. In light of the limitations of McAdams' model, namely the circularity and absence of clearly defined terms, the relationship between identity development and autobiographical reasoning remains a theoretical one. Before moving to a discussion of how autobiographical reasoning may relate to psychological functioning, it is important to note two additional factors that can influence the development of the life story: social reconstruction and culture.

\section{Influence of Social Interaction on the Development of the Life Story}

McAdams (1988; 1993) suggests that the existence of the life story does not need to be proven by being shared with others. Rather, the life story is an internalised narrative that can either be consciously or unconsciously created (McAdams, 1993). In reality, the life story is rarely recounted to others in its entirety (Habermas \& Bluck, 2000; Linde, 1993). More often, certain parts of the life story, or key autobiographical events, are shared with others for the purposes of developing connections and social bonds, to provide others with advice on the basis of one's own experiences or to explain or convey, important features or elements of one's personal past (Bluck \& Alea, 2009). Although the life story may not frequently be recounted to others in its entirety, initial evidence suggests that the majority of important life events are discussed with others. In one study, Thorne, McLean, and Lawrence (2004) asked young adults to write about three 'self-defining memories', defined as a memory of a specific event that has been thought about many times, elicits strong emotions, helps the participant understand who they are as a person and is more than one year old. Most (88\%) of self-defining memories reported by young adults in this study had previously been told to others.

Where elements of the life story and important life events are disclosed to others, the memory for these experiences can be influenced by the process of social construction (Marsh \& Tversky, 2004; Pasupathi, 2001). Sharing past experiences with others can alter the content of autobiographical narratives in a number of key ways. Listeners can actively attend to the story or appear disinterested, which can alter the amount of detail provided in the narrative (Pasupathi \& Hoyt, 2007). Listeners can also ask questions about the 
experience, which can influence or guide what information is elicited, and can challenge or offer a different perspective on events which can alter the manner in which the experience is perceived (McLean et al, 2007; Pasupathi, 2001).

\section{Influence of Culture on the Development of the Life Story}

In addition to the external processes of social construction, the life story may also be influenced by cultural norms and expectations regarding the form and content of the life story. Habermas and Bluck (2000) propose that the life story is constructed in accordance with cultural norms and expectations regarding what a prototypical life story will look like. Cultural norms and expectations regarding the prototypical life story have been variously defined as the cultural concept of biography, and the cultural life script (Berntsen \& Rubin, 2004; Bluck \& Habermas, 2000). These terms overlap conceptually to a significant degree. The 'cultural concept of biography' refers to the expectations held by members within a culture regarding what life events are expected to have the greatest impact on the individual, and thus most likely to be included in the life story, and at what age these events are likely to occur (Bluck \& Habermas, 2000). The extent to which a life story adheres to these cultural norms and expectations determines the cultural coherence of the story (Habermas \& Bluck, 2000). The cultural life script, on the other hand, refers to the actual list of life events, and timing of these events, that members of a culture expect to happen in a prototypical life (Berntsen \& Rubin, 2004). From a theoretical perspective, the cultural life script is unique to, and will differ greatly across, various cultures whereas the cultural concept of biography is a trans-cultural force that impacts the construction of the life story across all cultures.

Empirical work supports the existence of the cultural life script and, in Western cultures, has converged on 35 key life events that are likely to be experienced over the course of the lifetime (Rubin, Berntsen \& Hutson, 2008). According to key theorists (Bohn \& Berntsen, 2008), the cultural life script acts as a search engine that is activated when individuals are asked to tell their life story (or a part of the life story) and guides which life events are recalled in response to this request. While there is no guarantee that these events will be included in the life story for every individual, their shared cultural importance increases the likelihood that they will be included (Berntsen \& Rubin, 2004).

Habermas (2007), found only partial support for the notion that the cultural concept of biography is used to structure the life story. Habermas (2007) asked young adults to record the seven most important events an individual from their culture (German) are likely to experience in their lifetime. In a separate sample, young adults were asked to 
record the seven most important memories of their lives and to write about these in the form of a life story narrative. Comparisons between the lists of actually experienced important memories and the normative list of important memories revealed a relatively small degree of overlap between the two sets of events. That is, approximately one-quarter of personal memories matched the list of normative life events. In explaining this finding, Habermas (2007) suggests that while cultural norms may influence the inclusion of key events in the life story to an extent, the influence exerted by individual differences (such as personality) may also be strong.

In summary, the construction of the life story is influenced by a number of key factors. These include social reconstructive processes that influence how one's life story events are remembered and cultural norms which dictate which events are likely to have the greatest impact on an individuals life and thus should be included in the life story. The construction of the life story is also influenced by the manner in which the events are processed by the individual, namely through autobiographical reasoning. The remainder of this thesis is concerned with the question of whether and how autobiographical reasoning relates to psychological functioning during a lifetime period in which the development of identity is a significant concern; young adulthood. The following Chapter (Chapter Two) is dedicated to the discussion of research that relates more directly to the relationship between life story development and psychological functioning. 


\section{Chapter Two}

\section{Autobiographical Reasoning, Life Story Development and Psychological Functioning}

There is increasing evidence that the processes of autobiographical reasoning and life story development are associated with psychological functioning. The links between autobiographical reasoning, life story development and psychological functioning have been examined in three related branches of research. The first branch has focused on associations between the amount and complexity of autobiographical reasoning and psychological functioning. This branch is based on the theoretical notion that autobiographical reasoning serves an identity development function, which reflects positive psychological functioning during the adolescent/young adulthood periods. The second branch of research has examined the relationship between psychological functioning and the detection of positive meaning and/or positive outcomes from one's life events. The third branch has focused on the relationship between psychological functioning and valence of events (i.e. positive / negative events) which are included in the life story.

One of the major challenges in this field of research is the high degree of variance in the way psychological functioning is defined. Within this literature, psychological functioning has been conceptualised in one of three ways. First, poor psychological functioning has been conceptualised in terms of the presence of psychological distress, such as symptoms of depression (see McLean, Breen, \& Fournier, 2010). Second, positive psychological functioning has been conceptualised as components of psychological wellbeing described by the field of positive psychology e.g. self-esteem, optimism, satisfaction with life (McLean \& Breen, 2009; McLean \& Pratt, 2006). Third, positive psychological functioning has also been conceptualised as indicators of psychological maturity from the psychoanalytic perspective (Bauer, McAdams, \& Sakaeda, 2005).

Many studies investigating links between life story development and psychological functioning have used composite measures of 'psychological well-being' which combine symptoms of psychopathology (e.g. symptoms of depression) with indicators of positive adjustment (e.g. indicators of self-esteem; McLean, Breen, \& Fournier, 2010). While this approach has the benefit of providing a holistic view of psychological functioning, it may also be at risk of concealing differences between autobiographical reasoning and the different components of psychological functioning. 
A second limitation of this body of work is the diversity in the way autobiographical reasoning is conceptualised. In Chapter One, I described two conceptualisations of autobiographical reasoning; self-event connections and sophistication of meaning. The concepts of self-event connections and sophistication of meaning are well-defined and have demonstrated a reasonable level of reliability (McLean \& Fournier, 2008). Self-event connections and sophistication of meaning do, however, lack a degree of specificity in that the basic definition of these constructs do not describe the actual links that are made between past event and current self. That is, noting that an individual has made a self-event connection indicates that they have engaged in the process of autobiographical reasoning, but does not tell us anything about what the individual is actually saying about themselves in relation to the event. Similarly, a high sophistication rating shows that an individual has gained some sort of insight into themselves as a result of an experience, but does not tell us what that insight is.

Few attempts have been made to address the issue of specificity in self-event connections and sophistication of meaning ratings. In one study, McLean and Fournier (2008) asked younger (mean age $=21.4$ years) and older adults (mean age $=72.4$ years) to discuss three self-defining memories and coded these memory narratives for self-event connections. These authors then distinguished between the content of self-event connections, whether the connection described a trait, outlook, value or personal growth, and the valence of the connection, whether it was evaluated by the participant as positive or negative. Findings showed that self-event connections describing personal growth and values were more likely to be positively perceived than were self-event connections describing ones dispositions and outlook on life. While this study did examine the content of self-event connections, further links to psychological functioning were not explored.

Although self-event connections and sophistication of meaning are among the most commonly used measures of autobiographical reasoning, a number of additional measures have been created with a specific focus on positive self-descriptions. For example, themes of positive self-transformation are defined as statements which express openness towards exploring the effect of negative experiences on the self and describe positive outcomes from these experiences (Pals, 2006). In contrast, themes of differentiated processing refer to statements which suggest that life events are perceived as causing various forms of personal growth (Lilgendahl \& McAdams, 2011) ${ }^{2}$. While these conceptualisations have

2 These conceptualisations of autobiographical reasoning are discussed in more detail in Chapter Four 
been examined in relation to psychological functioning, they have either been shown to be fairly complex, amalgamating a number of different criteria such as openness and the detection of positive outcomes (Pals, 2006), or narrow in their focus solely on personal growth (Lilgendahl \& McAdams, 2011).

One consequence of these limitations is that research regarding the links between autobiographical reasoning, the life story and psychological functioning can appear difficult to reconcile. Where relevant, I have made connections between conceptualisations of autobiographical reasoning and psychological functioning used in individual studies, to highlight similarities in the approach taken by different researchers. It is important to note, however, that research examining links between autobiographical reasoning, the life story and psychological functioning is in early stages, and there is still much we do not know about the nature of these relationships (McLean \& Mansfield, 2010). Having outlined these limitations, I now turn to a discussion of the first branch of research, which has examined associations between the amount and complexity of autobiographical reasoning and psychological functioning.

\section{The identity function of autobiographical reasoning - links to psychological \\ functioning}

From a theoretical perspective, having a life story that is rich in autobiographical reasoning reflects healthy psychological functioning in young adulthood (McLean, 2005; 2008). This notion is derived from the combination of two key theoretical propositions discussed in Chapter One. The first proposition is that the construction of identity in adolescence and young adulthood is a developmental milestone one needs to attain in order to function well within society (McAdams, 1988; 1993). The second proposition is that identity is equivalent to the life story, and autobiographical reasoning is the core process through which the life story/identity is developed (Habermas \& Bluck, 2000; McAdams, 1988; 1993). Thus the presence of autobiographical reasoning in life stories constructed by adolescents and young adults may reflect positive psychological functioning, given the proposed drive for identity development during this lifetime period(McAdams, 2011).

Moderate support has been found for this claim. Blagov and Singer (2004) devised a measure of 'integrative memories', defined as life story narratives describing a lesson the individual derived from the event. This definition of integrative memories is similar to the conceptualisation of 'lesson learning' in McLean and Pratt's (2006) sophistication of meaning scale. Blagov and Singer (2004) conceptualised psychological functioning in terms of self-restraint, defined as multiple aspects of positive socialisation; impulse 
control, suppression of aggression, consideration of others and responsibility. According to these authors, moderate levels of self-restraint reflect adaptive psycho-social maturity as individuals with these levels of self-restraint demonstrate effective and flexible emotion regulation capabilities and can effectively socialise with others. Low and high levels of self-restraint were not considered to reflect adaptive psycho-social maturity as the former reflects difficulty regulating emotions and impulses while the latter reflects rigidity with regard to affect regulation. Blagov and Singer (2004) found that young adults (between 17 and 22 years of age) who exhibited moderate levels of self-restraint were more likely to construct narratives of self-defining memories containing lessons they had derived from the event relative to young adults who demonstrated maladaptive levels of self-restraint. This finding was interpreted as evidence that autobiographical reasoning (i.e. deriving lessons from one's life events) reflects emotional maturity in young adulthood.

In a more recent study, Tavernier and Willoughby (2012) found that for adolescents, engaging in autobiographical reasoning may be associated with improvements in psychological functioning over time. Tavernier and Willoughby (2012) asked adolescents (mean age $=17$ years, 52\% female) to write narratives of turning point experiences. Turning points were defined as "a major turning point in your life that changed the way you thought about something or how you behaved" (p. 1061). Each narrative was then coded for sophistication of meaning according to the system by McLean and Pratt (2006) outlined in Chapter One. Psychological functioning in this study was measured by reverse scored symptoms of depression, social anxiety, daily hassles, and self-esteem. Overall, findings showed that adolescents who reported learning a lesson or gaining insight from their turning point experienced better psychological functioning than adolescents who reported no meaning. Furthermore, sophistication of meaning predicted positive psychological functioning after controlling for baseline levels of functioning (i.e. prior to the turning point experience). Interestingly, psychological functioning before the experience of a turning point did not significantly predict whether lessons or insights were gained following the experience. These findings suggest that the presence of autobiographical reasoning in turning point narratives constructed by adolescents predicts positive changes in psychological functioning over time.

The relationship between autobiographical reasoning and psychological functioning has, however, been shown to be moderated by age. McLean et al. (2010) asked adolescent boys (aged 11 to 18 years) to write about four significant events from their life story; a high point, a low point, a turning point, and a continuity experience 
(defined as an experience in which the adolescent stayed the same). Each narrative was coded for self-event connections and sophistication of meaning. In a separate session, participants were asked to complete a measure of depression and a measure of self-esteem. These two measures were then combined to form a broad measure of 'psychological wellbeing'. Findings showed that regardless of age, boys who were more likely to construct narratives containing self-event connections that described self-change experienced higher levels of self-esteem and lower levels of depression than boys that were less likely to make these connections.

The relationship between sophistication of meaning and psychological well-being differed across age groups, however. For older adolescent boys, constructing life story narratives that contained a specific insight into the self (relative to lessons learnt or no/vague connections between the event and self) was associated with higher self-esteem and lower levels of depression. In comparison, for younger adolescent boys, constructing narratives containing insight into the self was associated with lower self-esteem and higher levels of depression. In explaining these findings, the authors suggested that the cognitive capacities involved in gaining insight into the self may not be developed until later adolescence, and that attempts to engage in this type of processing at an earlier stage may cause distress. In contrast, simply drawing connections between past experience and current self as highlighted by self-event connections may not rely on such advanced capacities and hence may be associated with well-being regardless of age.

It is important to note that the findings of McLean et al. (2010) do not highlight possible gender differences in the relationship between autobiographical reasoning and well-being during adolescence as girls were not included in the sample. In citing earlier evidence that parents tend to be more elaborative when having conversations with girls than they are with boys (Reese, Haden, \& Fivush, 1996), McLean et al. (2010) suggest that boys may not have developed the narrative skills to successfully engage in autobiographical reasoning during early adolescents. The fact that no differences are found in the amount or complexity of autobiographical reasoning in adulthood (see McLean, 2005; Pasupathi \& Mansour, 2006) suggests that these gender differences may cease to exist at some point during the transition from adolescence to adulthood.

Taken together, the research of Tavernier and Willoughby (2012) and McLean et al. (2010) provide preliminary evidence that, at least for older adolescents, learning lessons and gaining insight into the self from one's experiences reflects positive psychological functioning. These findings are supported by a broader body of work showing that the 
process of gaining insight into one's experiences more generally (i.e. not just in relation to the self) can play an active role in promoting physical and psychological health. Pennebaker and colleagues have devised an expressive writing task aimed at facilitating the process of gaining insight into one's experiences. In this paradigm, two groups of participants are asked to either write about their deepest thoughts and feelings about an important experience (expressive writing condition), or write about superficial topics (control condition) over a number of days (see Pennebaker, 1993; 1997 for a review).

Findings from this paradigm consistently show that young adults who demonstrate a deeper understanding of significant life events over the days of writing, experience better physical and psychological health than young adults who process events at a more superficial level. Specifically, within the expressive writing condition, participants who increasingly use words which denote insight (e.g. realise, understand, thought, knew) and causation (e.g. because, why, reason) over three to five days of writing show greater health improvements than participants who used these types of words at a consistent rate over time (Pennebaker \& Seagal, 1999 for a review). For example, university students who wrote about their transition to university (expressive condition) and who demonstrated an increase in the use of insight and causal words experienced better physical health (measured in terms of fewer health centre visits for illness related reasons) than students who evidenced no change in causal/insight words (Pennebaker \& Francis, 1996). Similar expressive writing tasks have shown to lower symptoms of depression among young adults (Gortner, Rude, \& Pennebaker, 2006) and increase marital satisfaction among soldiers recently exposed to combat (Baddeley \& Pennebaker, 2011).

Consistent with the findings of McLean et al. (2010), however, there is some evidence that the benefits of gaining a greater understanding of one's life events through expressive writing tasks is qualified by the age and developmental stage of participants. Fivush, Marin, Crawford, Reynolds, and Brewin (2007) assigned younger children (between 9 to 13 years of age) to an emotional writing condition in which they were asked to write about their deepest thoughts and feelings or a control condition in which they were asked how they would spend a typical day. Children in the emotional writing condition included more emotional and evaluative information in their narratives than did children in the control condition. Evaluations and explanations were associated with an increase in symptoms of anxiety among children in the emotional writing condition, however. In explaining these findings, the authors concluded that unstructured narrative tasks can 
increase psychological distress in children who may lack the regulatory and narrative skills to engage in adaptive reflection.

These findings have incited a wider discussion on some of the complexities in the relationship between autobiographical reasoning and psychological functioning (McLean \& Mansfield, 2010). The findings of McLean et al. (2010) and Fivush et al. (2007), in particular, highlight the importance of age and developmental status in considering the relationship between autobiographical reasoning and psychological functioning. In this thesis I proposed that another variable, whether autobiographical reasoning describes the self in positive or negative terms, significantly impacts the relationship between autobiographical reasoning and psychological functioning. This proposition was derived from the second branch of research which will now be discussed. This branch of research has investigated the relationship between psychological functioning and adaptive patterns of meaning found in life stories.

\section{Adaptive patterns of meaning making - links to psychological functioning}

The second branch of research examining links between autobiographical reasoning, the life story and psychological functioning is concerned with the relationship between positive psychological functioning and what have been collectively defined as adaptive patterns of meaning-making in life story narratives. The term 'meaning-making' is broadly used to refer to the process by which individuals describe, interpret and evaluate their life events and the self through the construction of the life story (McLean 2005; McLean \& Pratt, 2006; Singer, 2004). The notion of meaning making is extremely broad and is used to describe a large range of processes and, often, the outcomes of these processes (see Park, 2010 for the distinction between 'meaning-making' and 'meaningsmade'). Some of the processes subsumed under the general heading of 'meaning-making' and the products (i.e. meanings made) of these processes include: subjective appraisals or reappraisals regarding the meaning of life events; garnering an understanding of the cause of life events; perceptions of personal growth or positive changes in the self as a result of an event; changes in one's life goals or beliefs following an event; and a changed or greater sense of meaning in one's life (Park, 2010). As highlighted in this list, autobiographical reasoning (i.e. forming connections between past event and current self) is just one component of the broader concept of meaning-making.

Several studies have shown that describing positive meanings about events and/or

the self within the life story tends to be associated with positive psychological functioning (Davis, Nolen-Hoeksema, \& Larson, 1998; Grossbaum \& Bates, 2002; King, Scollon, 
Ramsey, \& Williams, 2000; McAdams, Reynolds, Lewis, Patten, \&Bowman, 2001). In an influential study, McAdams et al.(2001) asked mid-life adults (aged 35 to 65 years) to narrate eight events from their life story; a high point, low point, turning point, their earliest memory, an event from childhood, adolescence, adulthood and another important event. Each narrative was examined for themes of redemption and contamination. Themes of redemption were identified when, in the context of their life story narratives, participants described a negative situation or experience of negative emotional states that ended with a positive outcome. Themes of contamination were identified when participants described a positive situation or experience of positive emotional states that ended negatively.

McAdams et al. (2001) found that adults who were more likely to include redemption sequences life story narratives (summed across all event types) reported more satisfaction with life; higher self-esteem; were more likely to believe that they could understand, make sense of and manage life's challenges; and experienced lower levels of depression than adults who were less likely to include redemption sequences. In contrast, the inclusion of contamination sequences in adults' life story narratives was associated with lower levels of life satisfaction, lower levels of self-esteem, and higher levels of depression. In a related study, Grossbaum and Bates (2002) found that among midlife adults, contamination themes found across five life story events (high, low and turning points, and an early and another experience) predicted poor life satisfaction, while redemption sequences predicted three components of positive psychological well-being as measured by the Ryff scales (personal growth, self-acceptance, and positive relations with others).

One of the major challenges in the work of McAdams et al. (2001) and Grossbaum and Bates (2002), and, in fact, in all research adopting similar methodologies, is the measurement of meaning-making and psychological functioning using cross-sectional designs. As noted by Pasupathi (2012), capturing meaning-making and autobiographical reasoning at one point in time may provide an incorrect view of an individuals' tendency to draw positive and/or negative meanings from their life events. From a theoretical perspective, the meaning-making process is likely to be dynamic, with the actual meanings drawn from life events changing over time (Pasupathi, 2012). In addition to changes in meaning that may be expected as time since the event occurred elapses, changes over time may also be influenced by social constructive forces as the details of events are discussed with others (Pasupathi, 2001). Another limitation associated with measuring meaning- 
making and psychological functioning at one time point is that conclusions regarding causality between the two constructs cannot be made. In light of these limitations, the findings of McAdams et al. (2001) and Grossbaum and Bates (2002) provide some preliminary evidence that positive psychological functioning in adulthood is associated with the extent to which one's life events are narrated with positive or negative endings.

Also within this branch of research, the ability to describe benefits and positive outcomes from negative life events in particular appears to be a strong indicator of positive psychological functioning (Adler \& Poulin, 2009; King et al. 2000; McAdams et al. 2001; Pennebaker, Mayne, \& Francis, 1997; Stein, Folkman, Trabasso \& Christopher-Richards, 1997). MacCallum and Bryant (2008) asked bereaved individuals who were experiencing complicated grief, a maladaptive and debilitating set of grief symptoms experienced more than six months after the death, and bereaved individuals with no symptoms of complicated grief to describe three self-defining memories. Memories that were specifically related to the deceased individuals' death were coded for themes of redemption. Findings showed that narratives constructed by bereaved family members who were not experiencing symptoms of complicated grief were more likely to contain themes of redemption than were narratives constructed by bereaved individuals' experiencing complicated grief. In addition, none of the memory narratives provided by individuals experiencing complicated grief contained themes of redemption. These findings suggest that poor psychological functioning, defined in terms of complicated grief, was associated with a relative inability to infer any positive outcomes or aspects of the difficult experience.

Although MacCallum and Bryant (2008) did not define redemption themes as autobiographical reasoning per se, statements indicating redemption themes (e.g. realising personal strength and coping ability, forming better relationships with other family members) overlap with the construct of autobiographical reasoning. Overall, their findings highlight an important link between the way individuals reflect on and reason about negative life events and psychological functioning. From a theoretical perspective, negative events disrupt individuals' lives (e.g. in terms of their goals, relationships, values and activities) in a way that positive and neutral events do not (e.g. Lyubomirsky, Sousa \& Dickerhoof, 2006; Pals, 2006). As a consequence of this feature, negative events may require more explanation than do positive and neutral events, and these explanations may moderate the impact of these events for the individual (Baker-Ward, Eaton, \& Banks, 2005). Consistent with this notion, negative or challenging life events tend to contain 
more evidence of cognitive processing and autobiographical reasoning than do positive and neutral events (Bohanek, Fivush, \& Walker, 2005; McLean \& Pratt, 2006; McLean \& Thorne, 2003; Pals, 2006; Thorne et al.,2004).

More direct examinations of the link between autobiographical reasoning in the context of negative events and psychological functioning have shown that the ability to reason about positive outcomes for the self following challenging or difficult life experiences predicts positive psychological functioning in the years following the experience (King et al. 2000; Pals, 2006; Lilgendahl \& McAdams, 2011). From a theoretical perspective, Gillies and Neimeyer (2006) argue that positive psychological adaptation following a negative life experience, such as bereavement, is associated with engagement in three core processes. First, individuals are driven to 'make sense' of the experience, that is, to find a reason for why the event occurred. Second, positive adaptation is associated with the ability to find 'benefit' from the experience. Third, positive adaptation is associated with a change in 'identity', that is, shifts in one's view of the world and the self that can occur as a result of the search for meaning in negative experiences. Although not a direct test of this claim, one study by Pals (2006) suggests that at least the first two of these processes - the drive to make sense of the experience and the detection of benefit from the experience - are associated with positive psychological functioning in adulthood.

Pals (2006) asked 83 middle-aged women (52 years of age) to construct narratives of difficult life experiences that had occurred since leaving college. Each narrative was coded for themes of positive self-transformation, defined as statements expressing openness towards exploring the effect of the experience on the self, and statements describing positive outcomes from the negative experience. Nine years later, when the women were 61 years old, each participant underwent an interview with a clinician in which they were asked to discuss their thoughts and feelings on a number of topics such as work, family, relationships, health and aging. From these interviews, the authors constructed a measure of maturity (composite of clinician ratings regarding the participants self-understanding, integrity and emotional sophistication), and obtained participants' subjective appraisals regarding their level of satisfaction with their life and their physical health. Women who constructed narratives that contained themes of positive selftransformation at age 52 years scored higher on each indicator of psychological functioning (maturity, satisfaction with life, physical health) almost ten years later relative to women whose narratives did not contain themes of positive self-transformation. 
Pals' (2006) conceptualisation of positive self-transformation overlaps considerably with the notion of post-traumatic growth, which has also been discussed in relation to narrative identity and the life story (Pals \& McAdams, 2004). Post-traumatic growth has been described as a process by which a) individuals consider and explore the meaning of traumatic events and b) construe positive outcomes for the self as a result of the traumatic experience (Tedeschi \& Calhoun, 1996). These positive outcomes often include positive changes in the self, relationships with others and one's outlook or philosophy on life (Tedeschi \& Calhoun, 1996; 2004). Narrative may be one of the key ways through which post-traumatic growth develops as it enables individuals to construct and define the shift in their own words, while narrating the event within the context of the life story actively connects the growth with identity (Pals \& McAdams, 2004; Woodward $\&$ Joseph, 2003). Post-traumatic growth has been associated with adaptive coping and positive mental health following major illness (Park, Chmielewski, \& Blank, 2010), combat situations (Moore, Varra, Michael, \& Simpson, 2010), the 9/11 World Trade Centre attacks (Park, Riley,\& Snyder, 2012).

Autobiographical reasoning that describes personal growth in a more general sense (i.e. not solely as a product of traumatic events) has also been linked with positive psychological functioning. By definition, instances of autobiographical reasoning that describe personal growth link challenging or difficult life experiences with selfdevelopment and progression that are experienced as a direct result of the experience (McLean \& Fournier, 2008). Lilgendahl and McAdams (2011) conducted semi-structured life story interviews with 88 mid-life adults (mean age of 48 years). Each life story was transcribed and coded for themes of differentiated processing, the degree to which life events were perceived as causing various forms of personal growth. Participants also completed various measures of subjective well-being (satisfaction with life, positive and negative affect) and psychological well-being (self-acceptance, relations with others, autonomy, environmental mastery, personal growth, purpose in life). Overall, adults who incorporated more evidence of differentiated processing in their life story narratives experienced better subjective and psychological well-being than adults who were less likely to demonstrate these themes. When analysed by the valence of the life event (i.e. whether the event was positive or negative), however, findings showed that the relationship between differentiated processing and subjective / psychological well-being existed only in relation to negative experiences. 
In summary, there is increasing evidence that engaging in autobiographical reasoning is associated with positive psychological functioning in cases where the products of the reasoning process describe positive outcomes for the individual. This relationship between positive autobiographical reasoning and psychological functioning may be particularly evident when the reasoning process takes place in relation to negative life events. Returning again to the central theoretical premise of McAdams' Life Story Model of Identity, these findings have clear implications for the notion that identity is the life story. That is, an individual who constructs a life story that contains positive interpretations of their life events and/or links these events to positive characteristics of the self does, according to McAdams' model, have a narrative identity that is positive in nature. Having such a positively valenced life story, in turn, appears to be associated with a range of indicators of positive psychological functioning.

\section{Centrality of life story events and psychological functioning}

The third and final body of work that has investigated links between the life story and psychological functioning pertains to the nature of events that are included in the life story. As discussed in Chapter One, the life story is comprised of events and experiences that have personal significance to the individual (Pillemer, 1998; Singer \& Salovey, 1993). Individuals who experience psychological distress not only experience greater exposure to negative life events than others (Korkeila et al., 2010; LaNoue, Graeber, de Hernandez, Warner \& Helitzer, 2012), but also demonstrate a bias towards including negative life events in their life story that is not seen in non-distressed individuals (Habermas, Ott, Schubert, Schneider and Pate, 2008). If we assume that narrative identity is the life story (McAdams, 1988; 1993), then identity among psychologically distressed individuals may be comprised, to a greater degree than non-distressed individuals, of negative life events.

Even among individuals who experience an average or typical number of negative or stressful life events, including highly negative or stressful life events in the life story can be a risk factor for psychological distress (Berntsen \& Rubin, 2006; Berntsen, Willert, \& Rubin, 2003; Berntsen \& Rubin, 2007; MacCallum \& Bryant, 2008). This is especially true when these negative events are subjectively appraised as having more personal significance than other, more positive, experiences. In the study by MacCallum and Bryant (2008) described above, individuals who were suffering from complicated grief following the death of a loved one reported more self-defining memories relating to the deceased than did bereaved individuals who did not experience complicated grief. Additionally, Sutherland and Bryant (2005) found that trauma survivors who were 
experiencing post-traumatic stress disorder (PTSD) were more likely to recall memories of the trauma in response to a self-defining memory task than trauma survivors who did not experience PTSD. These findings suggest that for these highly distressed individuals, the experiences of loss or trauma were more likely to be included in the life story, and hence their narrative identity, than non-distressed individuals.

In 2004, Berntsen and Rubin introduced the idea that individuals perceive certain events as more central to their life story than others. These authors suggest that events which are central to one's identity and life story become a reference point for, and shape individuals understanding of, themselves and other life experiences. Perceiving negative events as being a central component of one's identity and life story has been shown to be a risk factor for psychological distress (Berntsen \& Rubin, 2006; Berntsen, Willert, \& Rubin, 2003). Berntsen \& Rubin, 2007) found that undergraduate students who endorsed traumatic experiences as central to their identity and life story exhibited more symptoms of Post-Traumatic Stress Disorder (PTSD) than did students who did not hold these beliefs. In a related study, Boals (2010) found that in a sample of university students, symptoms of depression and PTSD were positively correlated with beliefs about the centrality of negative events. These findings suggest that the extent to which individuals perceive negative events as a core part of their life story is an important predictor of psychological functioning.

\section{Justification for Current Studies}

The literature reviewed in this Chapter highlights a number of ways in which the development of the life story may be associated with psychological functioning. First, evidence of more advanced life story development, as measured by autobiographical reasoning, is associated with positive psychological functioning in older adolescence and young adulthood. Although not directly examined, this relationship may reflect more advanced identity development, which is a key developmental concern for adolescents and young adults. Second, adults who construct life stories which reflect positive meaningmaking and contain autobiographical reasoning that describes the self in positive ways experience better psychological functioning than adults whose life stories do not contain such positive meanings. Third, the inclusion, and centrality, of negative events in the life story may be a risk factor for poor psychological functioning. There are, however, a number of gaps in research supporting these claims, and a number of questions about the nature of the relationship between autobiographical reasoning, the life story and psychological functioning remain. 
First, we know very little about the relationship between psychological functioning and autobiographical reasoning that describes the self in positive ways in young adulthood as existing studies investigating these associations have focused solely on the autobiographical reasoning processes of adolescents, and mid-life and older adults. Given that the development of the life story (and identity) has been established as a key developmental concern for this age group, it is reasonable to expect similar patterns of findings in young adults to those found with mid-life and older adults. That is, with the increasing focus on autobiographical reasoning as a means of constructing information about the self, it is reasonable to expect that the patterns between psychological functioning and positively-toned autobiographical reasoning observed in adults may emerge, or be present, during young adulthood. Examining the relationship between autobiographical reasoning and psychological functioning in young adulthood is also important as a significant proportion of young adults experience symptoms of psychological distress such as depression and anxiety (Hankin et al., 1998). These experiences predict continuing mental health difficulties into adulthood and are associated with difficulty achieving social-developmental tasks such as the consolidation of identity (Howard, Galambos, \& Krahn, 2010; Vleioras \& Bosma, 2005).

Second, prior research has demonstrated a bias towards examining the relationship between psychological functioning and autobiographical reasoning that describes the self in positive ways. As outlined earlier, these definitions have centred on the constructs of positive self-transformation and personal growth. Comparatively less work has investigated the psychological functioning of individuals who attribute negative characteristics to the self through autobiographical reasoning. Given that the few measures of negative meaning-making that do exist within the literature predict poor psychological functioning (e.g. redemption themes; McAdams et al., 2001), it is reasonable to expect that negatively-toned autobiographical reasoning will similarly relate to poor psychological functioning in young adulthood.

Third, there is a lack of consensus within the literature regarding the best way to measure the construct of autobiographical reasoning valence, that is, the extent to which autobiographical reasoning describes the self in positive and negative ways. Existing definitions of positively-toned autobiographical reasoning (e.g. positive selftransformation) are complex, specific and on some occasions, demonstrate poor reliability. In contrast, self-event connections are easily defined and able to be reliably coded, but lack 
specificity in terms of what they can tell us about the nature of the links individuals are making between past event and current self.

To address these latter two concerns, the current thesis coded autobiographical reasoning valence in terms of positive, negative, neutral, and mixed (positive and negative) self-event connections. The conceptualisation of positive, negative, neutral and mixed self-event connections in the current thesis provides a novel and simplified way of capturing autobiographical reasoning valence that has three main benefits over existing conceptualisations. First, the inclusion of both positive and negative self-event connections allows both adaptive (positive) and potentially maladaptive (negative) forms of autobiographical reasoning to be captured. Second, positive self-event connections are not limited to a certain type of positive outcome for the self (e.g. 'positive selftransformation' or 'personal growth') and thus provide a more general measure of adaptive autobiographical reasoning. Third, positive and negative self-event connections can be coded in all narratives, regardless of the event type. This compares with previous indicators of positive autobiographical reasoning, which have largely been limited to the identification of positive outcomes or endings from negative events (see King et al., 2000; McAdams et al., 2001; Pals, 2006).

The operational definitions for positive, negative, neutral and mixed self-event connections were developed according to the original description of self-event connections provided by McLean and Fournier (2008). McLean and Fournier (2008) described selfevent connections as "any time when someone [makes] an explicit connection between the past and the self" (p. 529). In the current thesis, self-event connections were defined as positive when the connection referred to a positive characteristic of the self, mentioned personal growth, or denoted a positive evaluation of the self (e.g., "This event made me stronger"). Self-event connections were defined as negative when the statement referenced a negative characteristic of the self, or denoted a negative evaluation of the self (e.g., "this event is one of the main causes for the long term depression that I experience"). Self-event connections were defined as neutral when there was no evaluation of the self, or the connection was not clearly positive or negative (e.g., "this experience changed how I think about the world"). Self-event connections were coded as mixed when the connection involved elements of both positive and negative meaning. These were often situations where participants had learnt something (and thus could be considered an experience involving growth), but where the lesson learnt had negative connotations (e.g. "This experience taught me about the harsh realities of life"). 
Self-event connections were only recorded as positive or negative if: the selfcharacteristic highlighted was explicitly referred to as positive or negative by the participant (e.g., "It was a great experience because it taught me how to be independent" positive; "It has helped me over the last year, but in a negative way" - negative), or if it satisfied common cultural understandings of desirable and undesirable characteristics (e.g., "I gained a lot of self-confidence" - positive, and "This [event] has made me more paranoid" - negative).

The fourth point to make before outlining the details of the studies containing in this thesis is to acknowledge that there is a lot we do not yet know about the relationship between the development of the life story and psychological functioning. In reviewing the life story literature, McLean and Mansfield (2010) acknowledge that we do not yet know whether the development of the life story (through autobiographical reasoning) causes differences in psychological functioning, is the product of psychological functioning, or if there is a third variable that accounts for the association. Thus one of the key aims of this thesis was to undertake a number of studies that will increase our understanding of the relationships between autobiographical reasoning and life story development, and psychological functioning.

\section{The Aims of This Thesis}

This thesis broadly examines the relationship between psychological functioning and the valence of autobiographical present in the life stories of young adults. Four studies in this thesis were developed to address eight primary research aims:

1. To establish a relationship between psychological functioning and autobiographical reasoning valence in young adulthood (Studies 1a, 1b, 2, and 3).

2. To examine whether the relationship between autobiographical reasoning and psychological functioning is altered by young adults' tendency to reason about the self in positive and negative ways (Study 1a).

3. To examine whether the relationship between the centrality of negative events in the life story and psychological functioning is altered by young adults' tendency to reason about the self in positive and negative ways (Study 1a).

4. To explore the issue of directionality in the relationship between autobiographical reasoning valence and psychological functioning, in particular, whether autobiographical reasoning at one time point would predict changes in psychological functioning over time (Study 1b). 
5. To examine relationships between autobiographical reasoning valence and cognitive response styles; explanatory style, rumination, and cognitive reappraisal strategies (Study 2).

6. To explore whether cognitive response styles explain or alter the relationship between autobiographical reasoning valence and psychological functioning (Study 2).

7. To explore relationships between autobiographical reasoning and two aspects of the phenomenology of life story events; visual perspective and subjective distance (Study $3)$.

8. To explore whether autobiographical reasoning moderates key associations between psychological functioning and memory phenomenology (Study 3). 


\section{Chapter Three}

\section{Overview of Methodology}

Given the potentially burdensome task of eliciting entire life narratives (for both participants and researchers), life story research has tended to focus on eliciting narratives of key life events or experiences that are likely to be included in the life story, in favour of eliciting entire life narratives (Pillemer, 2001; McLean \& Thorne, 2003; Singer \& Moffitt, 1991-1992; Singer \& Salovey, 1993; Thorne, 2000; Thorne, McLean, \& Lawrence, 2004). One of the key strengths of this approach is that it enables researchers to examine patterns and themes present in narratives that are assumed to be present in entire life narratives, in an efficient manner. On the other hand, a key limitation of this approach is that the life story is mostly examined in a piecemeal fashion, rather than a holistic and complete way. This can mean that crucial information about how individuals structure and integrate life story events into an overarching narrative is lost (Bluck \& Habermas, 2000; Habermas and de Silveira, 2008). This limitation is not so important when the focus of research is on the core process through which the life story is developed, autobiographical reasoning.

\section{Methodology used to elicit life story narratives}

Across the three main studies in this thesis (Study 1a, Study 2, and Study 3), young adults were asked to write about three or four key events from their life story. Asking participants to recount a small number of key events from the life story is a popular approach in life story research as it enables the core process of autobiographical reasoning to be captured without placing the burden of recounting an entire life narrative on participants (McLean, 2005; 2008; McLean \& Pratt, 2008). The methodology used to elicit the key life story events in this thesis was adapted from Dan McAdams' guided autobiography tool (McAdams, 2006; available at http://www.sesp.northwestern.edu/foley/instruments/guided/). The full guided autobiography tool consists of ten 'critical episodes', defined as moments in an individual's life that stand out for some reason. The ten events are: high point, low point, turning point, a continuity experience (opposite of turning point), earliest memory, a childhood event (occurring before the age of 13 years), an adolescent event (occurring between the ages of 13 - 19 years) a morality experience (involving lessons of 'right and wrong'), an event involving a major decision and an event involving the achievement or working towards a currently valued goal. 
Consistent with the approach taken in the majority of autobiographical reasoning studies, the current research focused on three of these event types: high points, low points and turning points. In the first study (Study 1a; Chapter Four), participants were asked to narrate two high points and two low points from their life story. These two event types were chosen as they enabled comparisons to be made between the way young adults reasoned about the self in the context of both positive and negative life events. Participants were asked to narrate two events of each type (high and low points) to minimise the likelihood that differences in autobiographical reasoning by event type were not influenced by the actual event. That is, measuring autobiographical reasoning over two low points increases the likelihood of capturing an individual's general autobiographical reasoning tendencies than measuring it in relation to one single event. Following the completion of this first study, it became apparent that there were few differences in autobiographical reasoning amount, complexity and valence across the two events of each type. The methodology employed in the second and third studies were therefore modified.

In the second (Study 2) and third study (Study 3), participants were asked to narrate one high point, one low point and one turning point from their life story. The decision to include turning points followed the notion discussed by a number of key researchers, that autobiographical reasoning is likely to be a core feature of, and prevalent in, narratives of turning points. That is, turning point experiences are conceptualised as moments involving a change in the individuals' knowledge and understanding of themselves and/or the world (Tavernier \& Willoughby, 2012).

Another key methodological decision was whether to elicit verbal or written life story narratives. A decision was made to elicit written life story narratives in the current research for a number of reasons. As noted by Pasupathi (2001), life story narratives communicated to others are subject to social reconstruction. That is, a story that is told to others can change in terms of content and meaning, depending on the responses of the audience to which it is told. As a result of this, research adopting an oral method of eliciting life story narratives has been careful to give only the most basic and rudimentary prompts for gaining more detail in a narrated event (e.g. Pasupathi \& Mansour, 2006). Although these basic prompts are not likely to change the actual content of a story, it may encourage individuals to keep providing more information about an event than they otherwise would. It also raises the issue of when to stop prompting for more information 
and for whom and when to prompt, both factors which may reduce the standardisation and the reliability of the task.

\section{Description of the autobiographical narrative task}

The first step in the autobiographical narrative task was to inform participants of the demands of the task. Participants were first informed that they would be asked to "describe [three/four] key events or experiences that you've had in your life. These should be events or experiences you that might include in your life story". As part of this introductory information, participants were also told that they would be given a general description of the events they were expected to write about; however, within these general descriptions, they could choose the actual events they described and what details they chose to include in their narratives. Participants were then provided with a description of each type of event [high point, low point and turning point] and asked to write for as long as they liked about each of the events. Within their narratives of each event, participants were asked to consider how old they were when this event occurred, what happened in the event, who was involved, what they were thinking, feeling, why they think that this is an important event in their life story. They were also asked to consider what the event says about who they are, who they were, who they might be, and how they have developed over time. Consistent with (McAdams, 2006), the instructions for each of the event types were:

High points: "High points are defined as moments or episodes in a person's life in which he or she feels a sense of happiness, joy, excitement, contentment or some other positive emotion. These experiences vary widely and people report them to be associated with a whole range of experiences. A high point for you can be any experience that stands out in your memory as something that is/was extremely positive".

Low points: "Low points are moments or episodes in a person's life in which he or she feels extremely negative emotions such as despair, disillusionment, guilt, shame etc. A low point for you can be any experience that stands out in your memory as something that is/was extremely negative"

Turning points: "In looking back on one's life, it is often possible to identify certain key "turning points" - episodes in which a person undergoes substantial change. Turning points can occur in many different spheres of a person's life -in relationships with other people, in work and school, in outside interests, etc. 
We are especially interested in a turning point in your understanding of yourself"

"Please identify a particular episode in your life story in which you underwent an important transition or change with respect to your understanding of yourself. It is not necessary that you consciously saw the event as a turning point when it actually happened. Rather, what is important is that you now see this particular event as a turning point in your life. If you feel that you have experienced no dramatic turning points in your life, then describe a particular episode in your life that comes closer than any other to qualifying as a turning point"

Ethical approval for each of the studies conducted for this thesis was granted by the School of Psychology Ethics Committee at Victoria University of Wellington. A number of steps were taken to address any possible negative effects of the task on participants. First, participants were told that they could choose which events they wrote about before commencing the narrative task and were informed that they could withdraw from the study at any time. Second, all participants were provided with verbal debriefing at the conclusion of the testing sessions and were given a written debriefing information sheet that they took away with them when they had completed the study. This debriefing information described the nature and purpose of the study and participants were encouraged to ask any questions they had about the research. Participants were also given a list of support services they could contact if they were interested in seeking support for distress caused by life events that may or may not have been written about during the course of the study. 


\section{Chapter Four}

\section{Study 1a: The relationship between autobiographical reasoning processes and content, centrality of events and psychological functioning}

Study 1a was designed to address the first three aims of this thesis. The first aim was to establish a relationship between psychological functioning and autobiographical reasoning valence, as defined by positive and negative self-event connections. The second aim was to extend the findings of Tavernier and Willoughby (2012) and McLean et al. (2010), which suggest that the amount and complexity of autobiographical reasoning found in the life stories of older adolescents/young adults is associated with positive psychological functioning. Specifically, this study aimed to investigate whether the relationship between autobiographical reasoning (amount/complexity) and psychological functioning was altered by young adults' tendency to reason about the self in positive and negative ways. The third and final aim was to examine links between autobiographical reasoning valence and another component of life story development, the centrality of negative life events. Specifically, this study aimed to examine whether the relationship between the centrality of negative events in the life story and psychological functioning was altered by young adults' tendency to reason about the self in positive and negative ways.

This study formed the basis of a manuscript submitted for publication to the Special Issue of Memory: 'The Costs and Benefits of Finding Meaning in the Past' in 2012. This manuscript was accepted for publication in $2012^{3}$. This Chapter repeats much of the same information presented in Chapters One and Two. Although repetitive, this Chapter presents the information again as it is relevant for understanding the aims and hypotheses for the study.

\footnotetext{
3 Banks, M. V., \& Salmon, K.Reasoning about the self in positive and negative ways: Relationship to psychological functioning in young adulthood, Memory, (in press).
} 
When I was younger my Dad was diagnosed with a serious illness... eventually it got to the point where the doctors couldn't do anything more and he passed away. This event made me grow up well before I should have... Although the experience was tough for everyone, it has taught me that life is short and that I should never take people for granted... It has made me a lot mentally stronger, and I am able to cope with the more trivial things in life that other people my age see as a big deal.

Reflecting on the personal significance of our life events is a core component of life story development (McAdams, 1993, 1996; McLean, Pasupathi, \& Pals, 2007; Pillemer, 2001). This process of reflection is highlighted in the above excerpt from the life story of a young adult. In this example, the young adult describes how the death of their father led to the development of both personal characteristics (personal strength, coping skills) and the learning of valuable life lessons (life is short, not taking people for granted). The process of drawing connections between past events and the current self, as illustrated in this example, has been termed 'autobiographical reasoning' (Habermas \& Bluck, 2000).

Autobiographical reasoning is generally regarded as an indicator of psychological health and well-being (King, Scollon, Ramsey, \& Williams, 2000; Lilgendahl \& McAdams, 2011; McLean \& Breen, 2009; McLean, Breen, \& Fournier, 2010; Pals, 2006). Research supporting this claim is based on the theoretical notion that autobiographical reasoning is the key process through which the life story, and subsequently narrative identity, develops (McAdams, 1993, 2001; McLean, 2005, 2008; McLean \& Thorne, 2003; McLean et al., 2010; Pasupathi \& Mansour, 2006; Pasupathi, Mansour, \& Brubaker, 2007). This notion is closely aligned with early developmental theories (Erikson, 1968), which propose that identity formation is a key component of human development. Given the role of autobiographical reasoning in supporting the development of identity, evidence of this type of processing in autobiographical narratives is considered to be an indicator of normative, healthy development (Habermas \& de Silveira, 2008).

\section{Autobiographical reasoning and identity development}

Autobiographical reasoning has been operationalised in a number of ways. Two conceptualisations commonly referenced in narrative identity research are self-event connections and sophistication of meaning. Self-event connections are defined as explicit statements that describe a link between a personal experience and an aspect of the self such as personal traits, values, attitudes and beliefs (McLean \& Fournier, 2008; Pasupathi \& 
Mansour, 2006; Pasupathi, Mansour \& Brubaker, 2007). Sophistication of meaning is characterised as the level of insight or complexity of knowledge - particularly about the self - that is attained as a result of reflecting on one's past and self (Thorne, McLean, \& Lawrence, 2004). Both self-event connections and sophistication of meaning measure the extent to which information about the self is derived from life events, but they do so in slightly different ways. Self-event connections capture any explicit links a narrator has made between event and self, and therefore provide a basic measure of the extent to which autobiographical reasoning has occurred. Sophistication of meaning extends the measurement of this basic process by capturing the degree to which this knowledge describes abstract characteristics of the self or concrete behavioural lessons.

In support of the theoretical views discussed earlier, there is some, albeit indirect, evidence that both self-event connections and sophistication of meaning are related to the development of identity. Self-event connections are readily coded in narratives of memories that are considered self-defining (McLean, 2008) and statements depicting deeper insights into the self are commonly found in narratives of autobiographical memories that are shared with others for the purposes of self-explanation (McLean, 2005). Moreover, self-event connections and sophistication of meaning ratings tend to increase over adolescence, a crucial time for identity development (Habermas \& Paha, 2001; McLean \& Breen, 2009; Pasupathi \& Mansour, 2006).

\section{Autobiographical reasoning and psychological functioning}

There is, however, a paucity of research investigating how self-event connections and sophistication of meaning relate to psychological functioning. One recent study suggests that more self-event connections and more sophisticated meaning in narratives of important life events may relate to psychological functioning, but only for certain groups (McLean et al., 2010). McLean and colleagues asked adolescent boys (aged 11 to 18 years) to write about four significant events from their life story and coded each narrative for self-event connections and sophistication of meaning. Regardless of age, boys who constructed narratives containing more self-event connections that described self-change experienced higher levels of self-esteem, autonomy and lower levels of depression.

The relationship between sophistication of meaning and psychological well-being however, differed across age groups. For older adolescent boys, making statements indicative of deeper levels of insight into the self was associated with higher self-esteem and lower levels of depression. In comparison, for younger adolescent boys, more sophisticated meaning was associated with poorer psychological well-being. In accounting 
for these findings, the authors suggested that the cognitive capacities involved in gaining deeper insights into the self may not be developed until later adolescence, and that attempts to engage in this type of processing at an earlier stage may cause distress. In contrast, simply drawing connections between past experience and current self may not rely on such advanced capacities and hence may be associated with well-being regardless of age. Current thinking in relation to this body of research now favours the view that the relationship between autobiographical reasoning and psychological well-being is more complex than a simple "more is better" correlation (McLean \& Mansfield, 2010).

Understanding the relationship between autobiographical reasoning and psychological functioning may be enhanced by considering the findings of a broader body of work identifying adaptive patterns of autobiographical reasoning (see Bauer \& McAdams, 2004; Davis, Nolen-Hoeksema, \& Larson, 1998; McAdams, Reynolds, Lewis, Patten, \& Bowman, 2001; Pennebaker, Mayne, \& Francis, 1997; Stein, Folkman, Trabasso \& Christopher-Richards, 1997). This research shows that autobiographical reasoning describing personal growth and positive self-transformation as a result of challenging or difficult life experiences predicts positive psychological well-being in the years following the experience (King et al., 2000; Pals, 2006; Lilgendahl \& McAdams, 2011).

For example, Pals (2006) coded mid-life adults' narratives of difficult life experiences for themes of positive self-transformation, defined as statements expressing openness towards exploring the effect of the experience on the self, and describing positive outcomes from the negative experience. Narratives that contained themes of positive selftransformation predicted psychological well-being (maturity and satisfaction with life) and physical health almost ten years later. Similarly, Lilgendahl and McAdams (2011) found that themes of differentiated processing (the degree to which life events were perceived as causing various forms of personal growth) in life story narratives of mid-life adults were associated with subjective and psychological well-being. Although the precise conceptualisations of autobiographical reasoning differed across these two studies, these findings suggest that the extent to which autobiographical reasoning refers to the self in a positive manner is an important predictor of psychological well-being.

Taken together, the findings of the reviewed research suggest that both the extent to which individuals' engage in autobiographical reasoning, and the degree to which autobiographical reasoning is employed to link life experiences with positive outcomes or characteristics of the self, are important predictors of psychological well-being. 
Surprisingly, research has not yet examined whether, and how, these two components of autobiographical reasoning interact, nor whether the combination of these factors perhaps better predicts psychological well-being than either factor alone. Furthermore, while existing evidence suggests that reasoning about the self in positive ways is associated with positive psychological well-being, we know very little about the well-being status of individuals who, through the process of autobiographical reasoning, link past experience to negative characteristics or outcomes for the self. It is reasonable to expect that engaging in autobiographical reasoning will be associated with positive well-being when the connections drawn between event and self describe the self in positive ways, and associated with poor well-being when the connections describe the self in negative ways.

The current study aimed to address these gaps by first investigating the relationships between autobiographical amount/complexity, autobiographical reasoning valence (number of positive/negative self-event connections) and psychological functioning. Further to this, I examined whether the relationship between autobiographical reasoning (amount and complexity) and psychological functioning would be altered (moderated) by individual differences in the propensity to reason about the self in positive or negative ways. I predicted that for young adults who made a greater proportion of negative self-event connections, more, and more complex, autobiographical reasoning would be associated with poorer psychological functioning. For young adults who made a greater proportion of positive self-event connections, I predicted that more, and more complex, autobiographical reasoning would be associated with positive psychological functioning. The proportion of self-event connections that described the self in positive/negative ways were used as the moderating variables as this provided a measure of individual differences in the propensity to reason about positive/negative characteristics of the self that was independent of the number of connections made.

I examined the moderation effects in the context of narratives about negative life events (life story low points) and positive life events (life story high points) separately. Prior work shows that greater processing of negative, but not positive, life events is associated with subjective assessments of positive mental health status and satisfaction with life (e.g. Lyubomirsky, Sousa \& Dickerhoof, 2006). In accordance with these findings, I predicted that more, and more complex, reasoning would be related to psychological functioning (with the direction of the relationship explained by the proportion of positive/negative connections) in narratives of low, but not high points. 
The current study extended existing research in two additional ways. First, psychological functioning was conceptualised in terms of both the absence/presence of psychopathology and psychological well-being, and moderation analyses were conducted for these two measures separately. This approach is consistent with the notion that psychopathology and psychological well-being are related, yet discrete, constructs (see Ryff \& Keyes, 1995) and that positive psychological functioning comprises more than the absence of psychological distress (Bech, Olsen, Kjoller, \& Rasmussen, 2003). Second, I focused on young adulthood as this developmental stage is proposed to be a crucial time for the formation of identity and establishing a sense of self as an autonomous individual (Bauer \& McAdams, 2010; McLean \& Lilgendahl, 2008).

\section{Autobiographical reasoning and the centrality of events}

A further aim of the current study was to integrate research regarding adaptive patterns of autobiographical reasoning in the context of negative life events with a separate body of work investigating the psychological implications of incorporating negative events into the life story. From a theoretical perspective, psychological functioning can be jeopardised when negative events are perceived as being a central component of one's identity and life story (Berntsen \& Rubin, 2006; Berntsen, Willert, \& Rubin, 2003). Research in support of this claim is mixed, however. In one study, undergraduate students who endorsed traumatic experiences as central to their identity and life story exhibited more symptoms of Post-Traumatic Stress Disorder (PTSD) than did students who did not hold these beliefs (Berntsen \& Rubin, 2007). Similarly, symptoms of depression and PTSD were positively correlated with beliefs about the centrality of negative events in a sample of university students (Boals, 2010). Yet a recent study found no difference in the extent to which depressed and never-depressed individuals rated the centrality of negative events (Newby \& Moulds, 2011). Investigating individual differences in the way that individuals construct narratives about experiences that are central to identity may help explain these divergent findings.

Furthermore, given the theoretical role of autobiographical reasoning (both selfevent connections and sophistication of meaning) in the development of identity, it seems reasonable to expect more of this type of processing in relation to events that are subjectively appraised as more rather than less central to identity. This assumption raises questions about the relationship between autobiographical reasoning and psychological functioning, however. That is, if autobiographical reasoning is more prevalent in narratives of highly central negative events and, in turn, the centrality of negative events is 
associated with poorer psychological functioning, then we might expect the amount of autobiographical reasoning in narratives of highly central negative events to be associated with poorer psychological functioning. This scenario, however, does not take into account the way individuals' reason about themselves during this process of reflection. Consistent with the research reviewed earlier (e.g., Lilgendahl \& McAdams, 2011; Pals, 2006), it is possible that psychological functioning will not be compromised for individuals who make many positive connections to the self in the context of negative events that are perceived to be central to identity. I tested this hypothesis by examining whether the relationship between the centrality of life story low points and psychological functioning was moderated by individual differences in the propensity to reason about the self in positive ways. I predicted that endorsing low points as central to the life story and identity would not be associated with poor psychological functioning for young adults who tended to connect these experiences to positive characteristics or developments in the self. For young adults who were less likely to connect these experiences to positive characteristics of the self, I predicted that endorsing low points as central to identity would be associated with poor psychological functioning.

In summary, the aims of the current study were three-fold. First I aimed to clarify and extend previous research by investigating associations between the amount/complexity of autobiographical reasoning, autobiographical reasoning valence and psychological functioning. Second, I examined whether the relationship between autobiographical reasoning (amount and complexity) and psychological functioning was moderated by the extent to which young adults reasoned about positive or negative characteristics of the self in narratives of life story high and low points. Third I examined whether the extent to which young adults reasoned about positive characteristics of the self in narratives of life story low points moderated the relationship between beliefs about the centrality of these negative events and psychological functioning.

\section{Method}

\section{Participants}

Participants were 98 undergraduate students $(72 \%$ female $)$ enrolled in an introductory psychology course at Victoria University of Wellington, New Zealand. Participants received credit towards a course requirement of participation in research. Ages ranged from 17 to 28 years ( $M=19.4$ years, $S D=2.1$ years). Seventy percent of participants identified as New Zealand European/Pakeha, 17 percent as New Zealand 
Maori, 7 percent as Pasifika, and 21 percent as other (one in five participants identified with more than one ethnic background).

\section{Overview of Procedure}

Each session lasted for one hour and took place in psychology laboratories; participants (with numbers ranging between 2 and 8 per session) were seated at individual desks, separated by dividers. Participants read a summary of the nature and purpose of the study and provided consent. Following this, they completed a brief paper-based demographic questionnaire, measures of psychological distress and well-being, and an autobiographical narrative task through an online survey created by the researcher. The order in which the autobiographical narrative task and psychological functioning measures were completed was counterbalanced. The Centrality of Events Scale (Berntsen \& Rubin, 2006) was completed after each written narrative, also through the online survey tool.

\section{Tasks and measures}

\section{Psychopathology. Depression Anxiety Stress Scale (DASS; Lovibond \&}

Lovibond, 1995). This self-report questionnaire measures three related negative emotional states: depression, anxiety and stress, each on a 14-item subscale. Participants rate on a four-point scale how much each symptom applied to them over the past week $(0=$ Did not apply to me at all, $3=$ Applied to me very much, or most of the time). Consistent with previous research (Crawford \& Henry, 2003), a total DASS score was calculated by summing each of the subscales. Internal consistency for the DASS total score within the current sample was .97.

\section{Psychological well-being. Ryff's scales of psychological well-being (Ryff \&} Keyes, 1995). This self-report questionnaire measures six dimensions of psychological well-being; autonomy, environmental mastery, personal growth, positive relations with others, purpose in life, self-acceptance. Each subscale consists of 14 items, some which are positively and others that are negatively phrased. Participants record their level of agreement with each statement on a 6-point Likert scale ( $1=$ Strongly disagree, $6=$ Strongly agree). Consistent with previous research (see Bauer, McAdams, \& Sakaeda, 2005), a mean psychological well-being score was calculated by aggregating across each of the six sub- scales. Internal consistency for the composite well-being score in the current sample was .97 .

Autobiographical narrative task. Life story narratives were attained through an autobiographical narrative task. Participants were asked to provide written narratives of two high- and two low-points from their life story, modified from McAdams' guided 
autobiography and widely used in previous research (McAdams, 2006; available athttp://www.sesp.northwestern.edu/foley/instruments/guided/; see also McLean \& Breen, 2009; McLean \& Lilgendahl, 2008). High points were defined as "moments or episodes in a person's life in which he or she feels a sense of happiness, joy, excitement, contentment or some other positive emotion". Low points were defined as "moments or episodes in a person's life in which he or she feels extremely negative emotions such as despair, disillusionment, guilt, shame etc." Participants were asked to describe each of the two high and low points in some detail, and to consider the following in their descriptions: how old they were when the event occurred, what happened in the event, who was involved in the event, what they were thinking, feeling, and wanting in the event, why they think that this is an important event in their life story and what the event says about who they are. All participants provided their written narratives in the following order: high point, low point, low point, high point.

Centrality of narrated event. Centrality of Events Scale (7-item version) (CES7; Berntsen\& Rubin, 2006). This self-report questionnaire measures the extent to which a remembered event is central to an individual's personal identity, a reference point for the meaning imbued onto other remembered events, and their life story. After participants completed their written narratives, they were given the following instructions "Thinking about this event, please answer these questions by selecting a number from 1 to 5 ". Participants then recorded their level of agreement with a series of seven statements on a 5 -point Likert scale ( $1=$ totally disagree, $3=$ neither agree nor disagree, $5=$ totally agree). Questions included "I feel that this event has become part of my identity" and "This event has coloured the way I think and feel about other experiences". The short, 7-item centrality of events scale has been shown to have good psychometric properties (Cronbach's alpha $=.88$; Berntsen \& Rubin, 2006) and internal consistency for the current sample was .89.

\section{Narrative coding}

All written narratives were separated from their centrality ratings and coded by the first author. Twenty-two percent of narratives were coded by an independent coder who was blind to the hypotheses of the study. Inter-rater reliability (Kappas) are reported for each code.

Sophistication of meaning. Following McLean and Pratt (2006), each written narrative was coded according to a linear, 4-point scale, with higher scores reflecting greater complexity of meaning. A score of zero was given when there was no explanation 
of what the event meant for the individual. A score of 1 was given if the narrative included mention of a specific lesson the individual learnt from the event, which had implications for future behaviour. A score of 2 was given if the meaning of the event for the individual was mentioned, but vague in terms of detail. For example, narratives given a code 2 may have mentioned a change or growth in the self, but the specific details were not clear. Finally, a score of 3 was given to narratives that described how the individual gained a clear emotional, psychological, or relational insight into the self as a result of the event. Inter-rater agreement for the sophistication of meaning scale was kappa .80 .

Self-event connections. Following McLean and Fournier (2008), self-event connections were coded as any statements where participants explicitly linked an aspect of the narrated event to their sense of self. The number of self-event connections was calculated on a per narrative basis and inter-rater agreement for the total number of connections was kappa .81. Each self-event connection was coded for valence according to a coding scheme developed by the first author. For the purposes of reliability coding, self-event connections that were identified by both coders $(89 \%$ of connections identified by the first coder) were compared for valence. The coding scheme for valence consisted of four mutually exclusive categories ( $0=$ neutral connection; $1=$ =positive connection; $2=$ negative connection; $3=$ mixed positive and negative connection). Self-event connections were coded as positive when the statement referred to a positive characteristic of the self, mentioned personal growth, or denoted a positive evaluation of the self (e.g., "This event made me stronger"). Self-event connections were coded as negative when the statement referenced a negative characteristic of the self, or denoted a negative evaluation of the self (e.g., "this event is one of the main causes for the long term depression that I experience"). Self-event connections were coded as neutral when there was no evaluation of the self, or the connection was not clearly positive or negative (e.g., "this experience changed how I think about the world"). Self-event connections were coded as mixed when the connection involved elements of both positive and negative meaning. These were often situations where participants had learnt something (and thus could be considered an experience involving growth), but where the lesson learnt had negative connotations (e.g. "This experience taught me about the harsh realities of life").

Self-event connections were only recorded as positive or negative if: the selfcharacteristic highlighted was explicitly referred to as positive or negative by the participant (e.g., "It was a great experience because it taught me how to be independent" positive; "It has helped me over the last year, but in a negative way" - negative), or if it 
satisfied common cultural understandings of desirable and undesirable characteristics (e.g., "I gained a lot of self-confidence" - positive, and "This [event] has made me more paranoid" - negative). Inter-rater agreement for the valence of self-event connections was kappa .79.

\section{Results}

\section{Overview of analysis}

This section first presents descriptive statistics relating to key measures followed by correlation, regression and moderation analyses for each of the hypotheses. Second, results for the hypothesis that, reasoning about the self in positive and negative ways would moderate the relationship between autobiographical reasoning (amount and complexity) and psychological functioning are presented. Results for the hypothesis that reasoning about the self in positive ways would moderate the relationship between beliefs about the centrality of negative events and psychological functioning are presented third. As the focus of this second hypothesis was on negative life experiences, only data pertaining to low points is presented.

\section{Descriptive statistics}

On average, low point narratives $(M=410$ words, $S D=181$, range $=85-865$ words) were significantly longer than high point narratives $(M=361$ words, $S D=156$, range $=95-1,084$ words $;(97)=3.79, p<.05)$. To maximise consistency with previous research (McLean et al., 2010; Berntsen \& Rubin, 2006; Boals, 2010), the means and standard deviations for the narrative variables and centrality of event ratings are reported on a per narrative/event basis (Table 4.1). Of note, participants made more sophisticated meaning in narratives of high points than in narratives of low points $(t(97)=3.75, p<$ $.05)$, whereas the number of self-event connections was similar in narratives of low and high points $(t(97)=1.11, p>.05)$. The majority of self-event connections in narratives of high points described a positive characteristic/outcome for the self (92\%) and very few (3\%) described a negative characteristic/outcome for the self. Slightly more than half $(56 \%)$ of all self-event connections in narratives of low points described the self in a positive way while one-quarter (25\%) described the self in a negative way. Centrality ratings for high points and low points were similar $(t(97)=.13, p>.05)$ and both were considered highly central to identity. 
Table 4.1

Means and percentages for narrative variables and centrality ratings across memory type

\begin{tabular}{llccc}
\hline & & Low points & High points & Total \\
\hline 1. & Sophistication $(0-3)$ & $1.65(.96)$ & $2.00(.92)$ & $1.82(.82)$ \\
2. Number of self-event connections & $1.50(.99)$ & $1.60(.87)$ & $1.55(.83)$ \\
3. & Positive self-event connections (\%) & 56.0 & 91.8 & 74.1 \\
4. Negative self-event connections (\%) & 25.4 & 2.9 & 15.2 \\
5. Neutral self-event connections (\%) & 9.1 & 4.1 & 5.8 \\
6. Mixed self-event connections (\%) & 10.7 & 1.3 & 5.6 \\
7. Centrality of event (0-35) & $27.73(4.53)$ & $27.80(4.83)$ & $27.78(3.77)$ \\
\hline
\end{tabular}

Pearson correlations examined the associations amongst the key narrative variables (Table 4.2). Neither the amount (number of self-event connections) nor the complexity (sophistication) of autobiographical reasoning found across narratives of high and low points (combined) correlated significantly with total DASS scores or total RYFF scores (all $p s>.05)$. The number of positive self-event connections was inversely associated with total DASS scores, and positively associated with mean RYFF scores. In comparison, the number of negative self-event connections was positively associated with total DASS scores, and inversely associated with mean RYFF scores. These findings suggest that making more positive self-event connections is associated with lower levels of psychopathology and higher levels of psychological well-being, while making more negative self-event connections is associated with higher levels of psychopathology and poorer well-being. Controlling for verbosity (word count of narratives) did not alter the significance of any of these associations ( $r s=-.42$ to .40 , all $p s<.05)$. 


\section{Table 4.2}

Correlations amongst narrative variables and psychopathology/well-being scales

\begin{tabular}{|c|c|c|c|c|c|c|c|c|}
\hline & 1 & 2 & 3 & 4 & 5 & 6 & 7 & 8 \\
\hline 1. Sophistication & - & & & & & & & \\
\hline 2. Number of connections (average) & $.82 * *$ & - & & & & & & \\
\hline 3. Positive connections (average) & $.68 * *$ & $.88 * *$ & - & & & & & \\
\hline 4. Negative connections (average) & $.38 * *$ & $.35^{* *}$ & -.08 & - & & & & \\
\hline 5. Neutral connections (average) & .15 & .14 & .03 & -.13 & - & & & \\
\hline 6. Mixed connections (average) & .19 & $.35 * *$ & $.21^{*}$ & .06 & -.15 & - & & \\
\hline 7. Negative emotional states (DASS) & -.03 & -.11 & $-.28 * *$ & $.39 * *$ & -.16 & .00 & - & \\
\hline 8. Psychological well-being (RYFF) & .01 & .09 & $.31 * *$ & $-.40 * *$ & .06 & -.12 & $-.64 * *$ & - \\
\hline
\end{tabular}

$* p<.05, * * p<.01$ 


\section{Predictors of psychological functioning}

Regression analyses assessed which narrative variables (positive and negative selfevent connections) in which context (narratives of positive and negative life events) were the strongest predictor of psychopathology and psychological well-being. Total DASS and RYFF scores were entered separately as dependent variables. The numbers of positive, negative, neutral and mixed connections found in high points and low points were entered as predictor variables using the stepwise method. Two predictors (number of positive and negative connections in low points) explained $19 \%$ of the variance in DASS scores $\left(R^{2}=.19, F(2,95)=11.04, p<.01\right)$. That is, young adults' experiences of psychopathology were predicted by higher numbers of negative connections and lower numbers of positive self-event connections in narratives of low points. Three predictors (number of negative connections in low points, number of negative and positive connections in high points) explained $24 \%$ of the variance in RYFF scores $\left(R^{2}=.24, F(3,94)=9.93, p<.01\right)$. That is, fewer negative connections in narratives of high and low points and higher numbers of positive connections in narratives of high points predicted young adults' psychological well-being. Significant predictors are shown in Tables 4.3 and 4.4.

Table 4.3

Stepwise regression of narrative variables in the prediction of DASS scores

\begin{tabular}{lccc}
\hline \multicolumn{1}{c}{ Predictors of psychological distress } & $B$ & $S E B$ & $\beta$ \\
\hline (Constant) & 35.05 & 4.70 & \\
Number of negative connections in low points & 15.34 & 4.71 & $.31^{* *}$ \\
Number of positive connections in low points & -9.30 & 3.48 & $-.25^{* *}$ \\
\hline
\end{tabular}

Notes: $R^{2}=.19,(p s<.05),{ }^{*} p<<.01$ 
Table 4.4

Stepwise regression of narrative variables in the prediction of RYFF scores

\begin{tabular}{lccc}
\hline \multicolumn{1}{c}{ Predictors of psychological well-being } & $B$ & $S E B$ & \multicolumn{1}{c}{$\beta$} \\
\hline (Constant) & 59.16 & 1.89 & \\
Number of negative connections in low points & -5.66 & 1.58 & $-.34^{* *}$ \\
Number of negative connections in high points & -10.80 & 4.56 & $-.22^{* *}$ \\
Number of positive connections in high points & 3.11 & 1.10 & $.26^{* *}$ \\
\hline
\end{tabular}

Notes: $R^{2}=.24,(p s<.05), * * p<.01$

\section{Moderation of relationship between autobiographical reasoning and psychological functioning, by autobiographical reasoning valence}

Moderation analyses were conducted with the number of self-event connections and sophistication of meaning as main effects, proportion of positive/negative connections (relative to total) as the moderating variables, and psychopathology (DASS scores) and psychological well-being (RYFF scores) as the outcome variables. Only those participants who made at least one self-event connection in low point narratives $(\mathrm{n}=85)$ and high point narratives $(n=90)$ could be included in the analyses. As neutral and mixed connections were not significantly associated with DASS or RYFF scores, only the proportion of positive (relative to total) connections and proportion of negative (relative to total) connections were included as moderating variables. Moderation effects for low points are presented first, followed by high points.

Narratives of life story low points. Amount/complexity of autobiographical reasoning and psychopathology (DASS). Hierarchical regression analyses first tested the hypotheses that the proportion of positive/negative connections would moderate the relationship between amount/complexity of autobiographical reasoning and DASS scores in the context of life story low points. In the first set of analyses, number of self-event connections and sophistication ratings for narratives of low points were entered (separately) as main effects. The proportion of negative self-event connections were also entered as a main effect. The two-way interaction terms (number of connections $\mathrm{x}$ proportion negative connections and sophistication $\mathrm{x}$ negative connections) were entered in the second step. There were no main effects for number of self-event connections $(\beta=$ $.01, p>.05)$ or sophistication of meaning $(\beta=.11, p<.05)$; however, in both analyses, there were significant main effects for the proportion of negative self-event connections 
$(\beta s>.34, p s<.05)$, confirming the basic relationship that reasoning about the self in negative ways was associated with higher DASS scores. There was also a significant interaction between number of connections and proportion negative connections $(\beta=.25, p$ $<.05)$, but no significant interaction between sophistication and negative connections at the $5 \%$ level $(\beta=.20, p=.06)$.

To aid interpretation of these results, the interactions were graphed using ModGraph (Jose, 2004), a purpose-built tool for analysing moderation effects. Simple slope statistics (Figure 4.1) revealed that in both sets of analyses, the slope for the high proportion of negative connections was significantly different from zero (slopes $>10.09$, ps $<.05)$, while the slopes for medium and low proportion of negative connections were not different from zero $(\text { slopes }=-9.17 \text { to } 4.28, p s>.05)^{4}$. This finding suggests that for young adults who were more likely to link low point experiences to negative characteristics of the self, making more self-event connections and more sophisticated meaning in narratives of these experiences was associated with higher DASS scores.
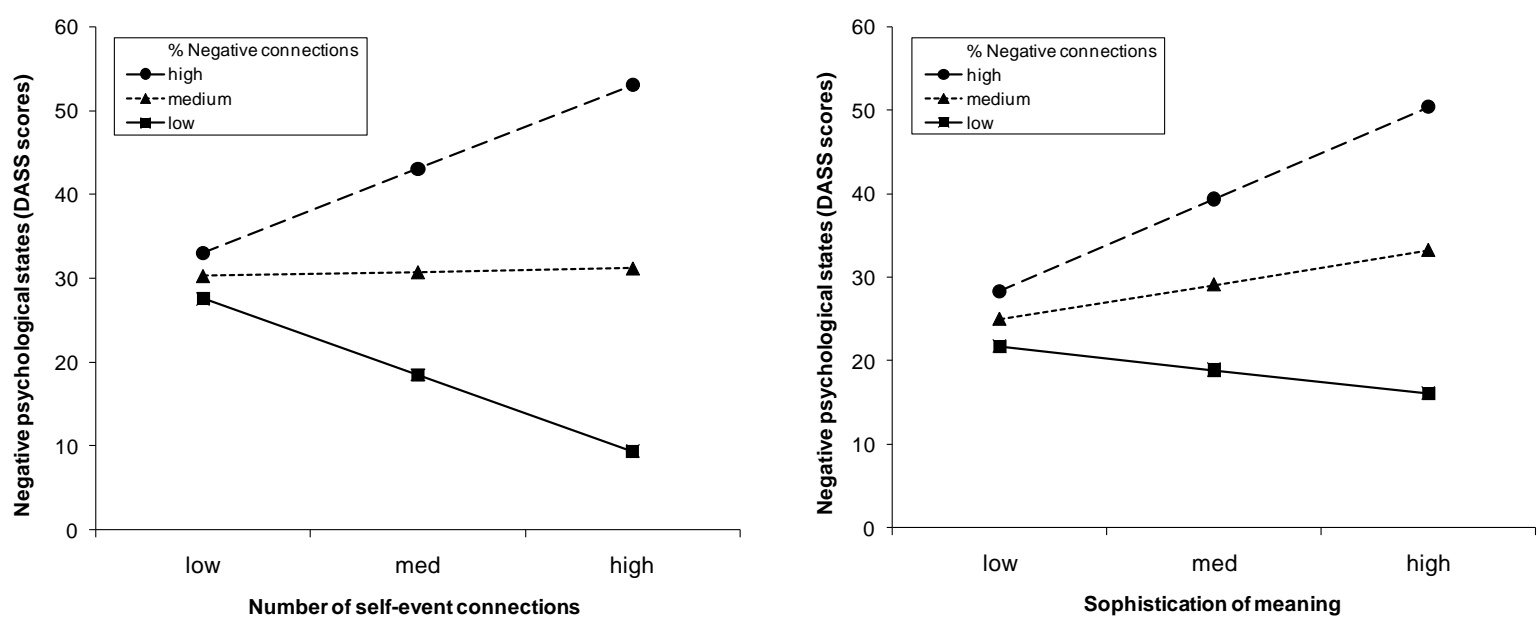

Figure 4.1. Moderation of the relationships between number of self-event connections, sophistication of meaning and DASS scores by proportion of negative self-event connections in narratives of low points

\footnotetext{
${ }^{4}$ Note, the slope for low proportion of negative self-event connections in the self-event connections to DASS relationship was significant at the $10 \%$ level $(p=.08)$
} 
These analyses were then repeated for the proportion of positive self-event connections. Consistent with the above analysis, there were no main effects for number of self-event connections or sophistication of meaning $(\beta s<.16$, $p s>.05)$, but there were significant main effects for the proportion of positive self-event connections ( $\beta s>-.34$, ps $<.05)$, confirming the basic relationship that reasoning about the self in positive ways was associated with lower DASS scores. A significant interaction was detected between number of self-event connections and proportion of positive connections $(\beta=-.21, p<.05)$ but not between sophistication and positive connections $(\beta=-.16, p>.05)$. Further analysis with ModGraph showed that the proportion of positive connections moderated the relationship between DASS scores and sophistication of meaning, but not self-event connections. Simple slope statistics revealed that the slope between sophistication and DASS scores for young adults who made the lowest proportion of positive connections was significantly different from zero $($ slope $=12.06, t(81)=2.32, p<.05$, remaining slopes $=0.46$ to $6.26, p s>.05)$. Although in the expected direction, none of the slopes between number of self-event connections and DASS scores were significantly different from zero (slopes $=-5.89$ to $9.39, p s>.05$ ). These findings suggest that for young adults who were the least likely to connect low point experiences to positive characteristics of the self, more sophisticated meaning in narratives of low points, but not more self-event connections, was associated with higher DASS scores.

\section{Amount/complexity of autobiographical reasoning and psychological well-being} (RYFF scores). The above analyses were repeated with RYFF scores as the outcome variable ${ }^{5}$. In contrast to the above findings for the DASS, significant moderation effects were detected for the proportion of positive, but not negative self-event connections. Simple slope analyses (Figure 4.2) revealed that for young adults who were the least likely to connect low point experiences to positive characteristics of the self, making more selfevent connections and more sophisticated meaning in narratives of low points was associated with lower RYFF scores (slopes $<-3.19$, ps $<.05$; remaining slopes $=-2.20$ to 0.92 , ps $>.05$ ). Young adults' propensity to connect low point experiences to negative characteristics of the self did not alter the relationship between self-event connections/sophistication of meaning and RYFF scores (slopes $=-2.89$ to 1.34 , ps $>.05$ ).

\footnotetext{
${ }^{5}$ In the interest of preserving space, the results for the regressions associated with these analyses are not discussed.
} 
Narratives of life story high points. Each of the above analyses were then repeated for narratives of life story high points. In contrast to the findings for narratives of low points, no significant moderation effects were detected for high points. That is, young adults' propensity to make positive/negative self-event connections in narratives of life story high points did not alter the relationships between the amount (self-event connections) and complexity (sophistication) of autobiographical reasoning and DASS scores (all slopes $=-6.43$ to 4.21 , ps $>.05$ ), nor did it alter the relationships between amount/complexity of autobiographical reasoning and RYFF scores (all slopes $=-1.72$ to $3.26, p s>.05)$.
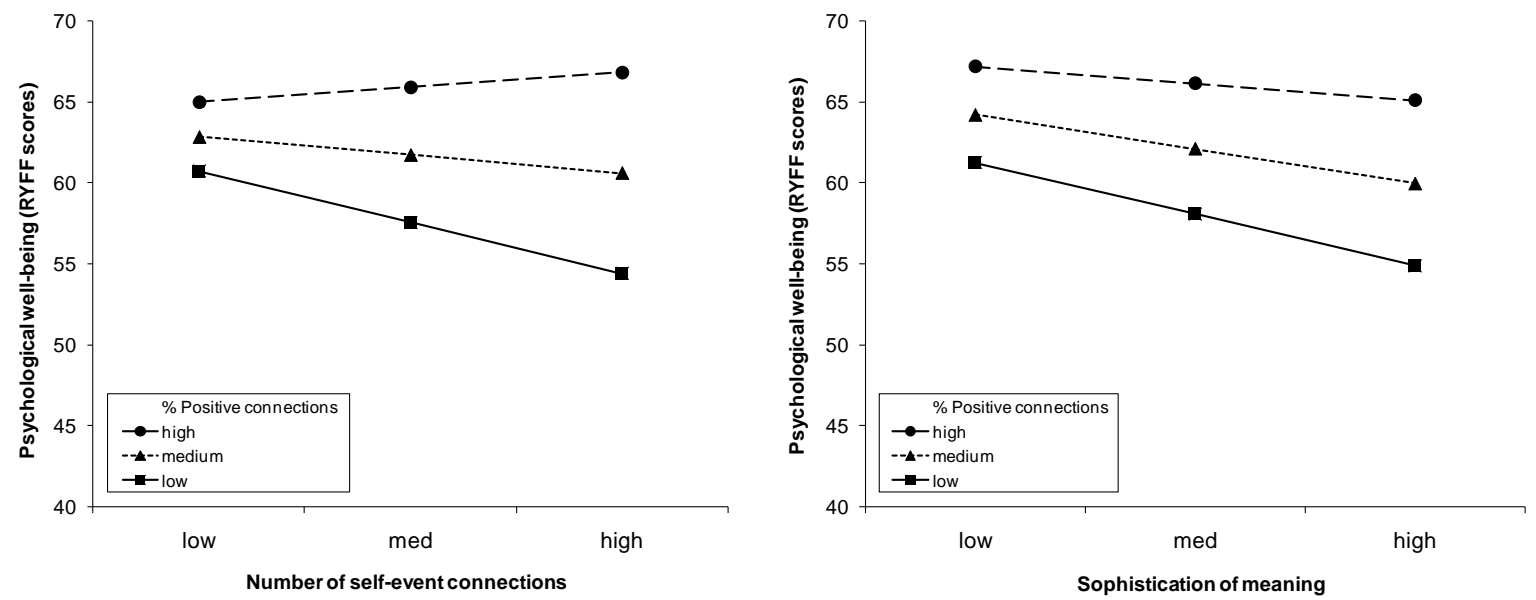

Figure 4.2. Moderation of the relationships between number of self-event connections, sophistication of meaning and RYFF scores by proportion of positive self-event connections in narratives of low points

In summary, reasoning about the self in positive/negative ways moderated the relationship between amount/complexity of autobiographical reasoning and psychological functioning in relation to low, but not high, points. For young adults who demonstrated the strongest tendency to make negative self-event connections in narratives of low points, making more self-event connections and more sophisticated meaning was associated with higher DASS scores (psychological distress). Furthermore, for young adults who were the least likely to make positive self-event connections in narratives of low points, making more self-event connections and more sophisticated meaning was associated with lower RYFF scores (poorer psychological well-being). The extent to which young adults reasoned about the self in positive or negative ways did not alter the relationships between amount/complexity of autobiographical reasoning and psychological functioning. 
Moderation of relationship between centrality of event ratings for low points and psychological functioning, by autobiographical reasoning valence

Pearson correlations first examined the associations amongst the narrative variables, centrality ratings for high and low points, and DASS and RYFF scores (Table 4.5). Centrality ratings for low and high points were positively associated with both sophistication of meaning and number of self-event connections. This suggests that narratives containing more, and more complex, autobiographical reasoning were more likely to be considered central to identity. Participants who rated low points as more central to identity made proportionately more negative (relative to total) connections than participants who rated low points as less central to identity. Centrality of event ratings for low points were positively associated with DASS scores but not significantly related to RYFF scores, suggesting that centrality of event ratings for low points is a significant predictor of psychopathology but not psychological well-being.

Table 4.5

Correlations between centrality ratings for high and low points, narrative variables and psychopathology/well-being scales

\begin{tabular}{llcc}
\hline & & \multicolumn{2}{c}{ Centrality of event ratings } \\
\cline { 3 - 4 } & & Low points & High points \\
\hline 1. & Sophistication & $.31^{* *}$ & $.27^{* *}$ \\
2. & Number of self-event connections & $.30^{* *}$ & $.34^{* *}$ \\
3. & Positive self-event connections (\%) & -.14 & .06 \\
4. & Negative self-event connections (\%) & $.24^{*}$ & -.09 \\
5. & Neutral self-event connections (\%) & .02 & -.02 \\
6. & Mixed self-event connections (\%) & -.12 & .04 \\
7. & Negative emotional states (DASS) & $.30^{* *}$ & .03 \\
8. & Psychological well-being (RYFF) & -.13 & .09 \\
\hline
\end{tabular}

$* p<.05, * * p<.01$ 
Hierarchical regression analyses investigated the hypothesis that reasoning about the self in positive ways in the context of negative life events would moderate the relationship between the centrality of these events and DASS and RYFF scores. Moderation analyses were conducted with data from $n=85$ participants who made at least one self-event connection in their narratives of low points. In each regression, the two main effect variables - centrality of event ratings for low points and the proportion of positive self-event connections in low point narratives - were entered in the first step and the two-way (centrality x proportion of positive connections) interaction term was entered in the second step. In each set of analyses, significant main effects for proportion of positive self-event connections $(\beta s=-.32$ to $.43, p s<.05$.) and significant (centrality $\mathrm{x}$ positive connections) interactions $(\beta s=-.25$ to $.29, p s<.05)$ were found. There was also a significant main effect for centrality of low points on DASS scores $(\beta=.23, p<.05)$ but not RYFF scores $(\beta=-.05, p>.05)$,

Further analysis of these interactions with Modgraph revealed slightly different moderation effects for DASS and RYFF scores (Figure 4.3). Simple slope statistics revealed that the slopes between centrality ratings and DASS scores were significantly different from zero for young adults who made the lowest proportion of positive self-event connections (slope $=3.15, t(81)=5.04, p<.05$ ) and medium proportion of positive selfevent connections $($ slope $=1.60, t(81)=2.34, p<.05)$. This finding suggests that for young adults who were the least likely, and who were moderately likely to connect low point experiences to positive characteristics of the self, greater centrality of event ratings were associated with higher DASS scores. The slope for high proportion of positive selfevent connections was not significantly different from zero(slope $=0.05, t(81)=0.07, p>$ $.05)$, suggesting that for young adults who demonstrated the strongest tendency to connect low point experiences to positive characteristics of the self, greater centrality of event ratings for low points were not associated with higher DASS scores.

Simple slope statistics also showed that the slopes between centrality ratings and RYFF scores were significantly different from zero for young adults who made the lowest proportion of positive self-event connections (slope $=-0.69, t(81)=-3.23, p<.05)$ and the highest proportion of positive connections ( slope $=0.47, t(81)=2.20, p<.05$ ). The slope for medium proportion of positive self-event connections was not significantly different from zero (slope $=-0.11, p>.05$ ). These findings suggest that greater centrality of event beliefs for low points were associated with higher RYFF scores for young adults who were 
most likely to make positive self-event connections, and were associated with lower RYFF scores for young adults who were least likely to make positive self-event connections.
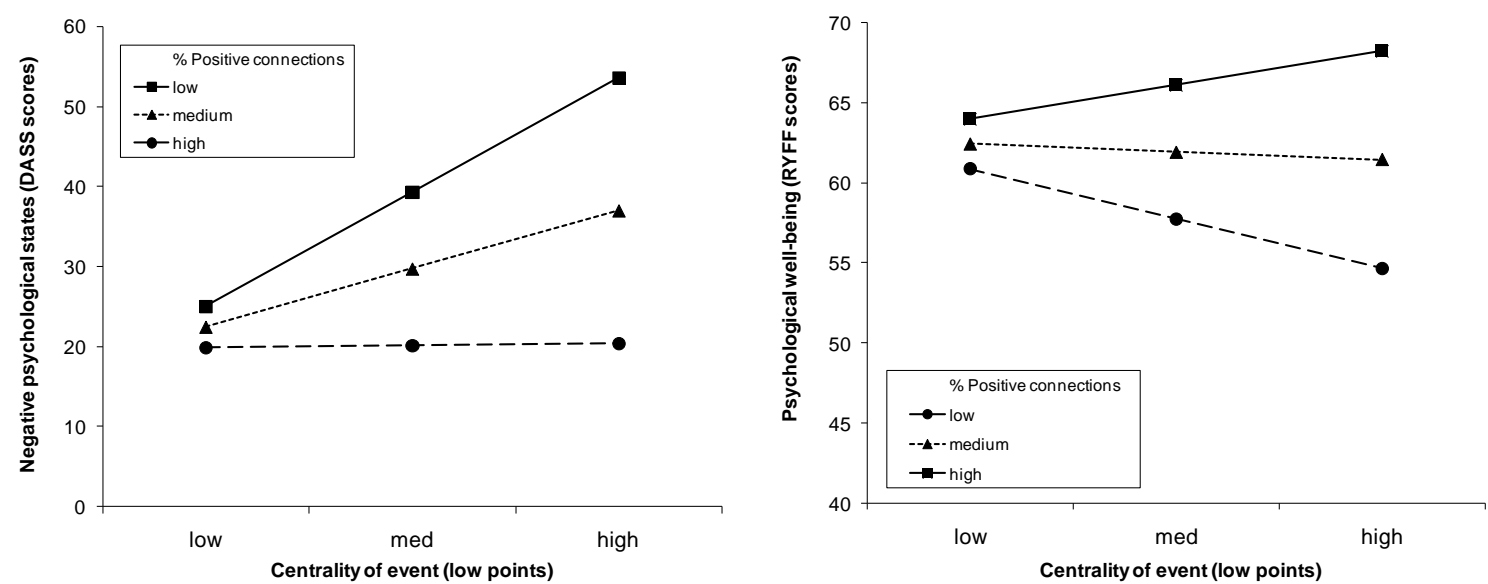

Figure 4.3. Moderation of the relationship between DASS scores, RYFF scores and centrality of event ratings for low points by proportion of positive self-event connections in narratives of low points

In summary, endorsing life story low points as central to identity and the life story was associated with higher levels of psychopathology and poorer psychological wellbeing, but not for young adults who were more likely to make self-event connections that described the self in positive ways in narratives of these experiences. For young adults who were the most likely to make positive self-event connections in narratives of low points, greater centrality of event beliefs were unrelated to psychopathology, and were associated with an increase in psychological well-being.

\section{Discussion}

The aims of the current study were three-fold. The first aim was to clarify the relationships between amount/complexity of autobiographical reasoning, autobiographical reasoning valence (positive/negative self-event connections) and psychological functioning. The second aim was to investigate whether the individual differences in the propensity to make positive or negative self-event connections in narratives of high and low points moderated the relationship between autobiographical reasoning (amount and complexity) found in these narratives, and psychological functioning. The third and final 
aim was to explore whether the relationship between psychological functioning and the centrality of negative events was moderated by individual differences in the propensity to make positive self-event connections in narratives of these experiences.

With respect to the first aim, findings showed that neither total number of selfevent connections nor sophistication of meaning across narratives of high and low points (combined) was associated with psychopathology (DASS scores) or psychological wellbeing (RYFF scores). Earlier findings regarding these relationships are mixed. For example, McLean et al. (2010) found a positive relationship between sophistication of meaning and a combined measure of self-esteem and reverse scored depression for older adolescents. Yet an earlier study found no relationship between sophistication of meaning and self-esteem for older adolescents (McLean \& Breen, 2009). The measures used in the current, and previous, studies did however, differ slightly (self-esteem and depression versus a broader measure of psychopathology and psychological well-being). While low self-esteem is associated with psychopathology (Trzesniewski et al., 2006), the relationship is somewhat controversial (Baumeister, Campbell, Krueger, \& Vohs, 2003). Thus the extent to which similar patterns of associations might be expected between autobiographical reasoning and these various measures is uncertain. Overall however, the current findings suggest that the core processes of autobiographical reasoning (amount and complexity) are not associated with psychopathology and psychological well-being in young adulthood.

Both the number of self-event connections and sophistication of meaning ratings were similar across narratives of high and low points. This finding is consistent with earlier work (McLean, et al., 2010) and suggests that young adults may draw equally on high and low point events for identity development (McLean \& Lilgendahl, 2008). It is also important to note that the number of self-event connections in participants' narratives was slightly lower than that recorded in earlier work with younger adults (McLean \& Fournier, 2008), possibly reflecting differences in the instructions used to elicit life story narratives. Writing about 'self-defining memories' as employed in these previous studies (McLean \& Fournier, 2008; Singer \& Moffit, 1991-1992) may highlight linkages between event and self more so than does than writing about high and low points from the life story. One limitation of the current study was that gender differences could not be examined in any of the effects due to small group numbers. Earlier work has highlighted gender differences in the length and detail of autobiographical narratives, and in the qualities of autobiographical reasoning found in autobiographical narratives (McLean \& 
Breen, 2009; Zaman \& Fivush, 2011). Further research comparing the relationships between the components of autobiographical reasoning examined in the current study and psychological functioning for males and females is required.

Also with respect to the first aim, findings highlighted differences in the association between the valence of autobiographical reasoning found in narratives of high and low points and the two measures of psychological functioning. Young adults who made more positive self-event connections and fewer negative self-event connections in narratives of low points experienced lower levels of psychopathology than did young adults who made fewer positive, and more negative, connections in relation to low points. In comparison, young adults who made fewer negative self-event connections in narratives of low and high points, and more positive connections in high points experienced higher levels of psychological well-being than did young adults who made more negative connections in low and high points, and fewer positive connections in high points. These previously untested associations suggest that autobiographical reasoning valence is a valid predictor of psychological functioning in young adulthood, but that the exact nature of the relationships may depend on whether the narrated event is positive or negative. These findings raise the possibility that psychological well-being is associated with the way young adults process information about the self in both positive and negative experiences, whereas psychopathology is associated primarily with the way information about the self is processed in relation to negative experiences.

\section{Does autobiographical reasoning valence moderate the relationship between amount/complexity of autobiographical reasoning and psychological functioning?}

With respect to the second aim, the question of whether individual differences in the propensity to make self-event connections that described the self in positive or negative ways would moderate the relationship between autobiographical reasoning (amount and complexity) and psychological functioning was explored. Individual differences in the propensity to make positive/negative self-event connections were calculated as the number of positive/negative self-event connections relative to total connections made.

Significant moderation effects were detected in relation to narratives of low, but not high points. Consistent with our predictions, for young adults who showed the strongest tendency to link their experiences to negative characteristics of the self, making more self-event connections and describing deeper insights into the self in narratives of low points was associated with higher levels of psychopathology. This pattern was not 
evident for young adults who showed a moderate or little tendency to reason about the self in negative ways.

Although the correlational nature of our analysis prevents firm conclusions being drawn, there are at least three possible explanations as to why more, and more complex, autobiographical reasoning in the context of low points was associated with higher levels of psychopathology for young adults who reasoned about the self in predominantly negative ways. First, from a constructionist perspective (see McLean et al., 2007; Pasupathi, 2001) forming a narrative whereby the self is assigned negative attributes may encourage the narrator to believe that they hold these attributes which, in turn, may induce negative emotional experiences and symptoms of psychopathology. A second perspective is that young adults who experience high levels of psychopathology may simply be more likely to reflect on the negative aspects of themselves when asked to narrate a low point from their life story. This interpretation is consistent with research showing that individuals who experience higher levels of psychological distress are more likely to favour negative views of the self and show a reduced capacity to derive positive information about the self from autobiographical memory (Blaney, 1986; Gara et al., 1993; Habermas et al., 2008; Mansell \& Clark, 1999; Swales, Williams, \& Wood, 2001; Williams et al., 2007). A third, and perhaps most likely, possibility is that both of these scenarios exist in unison. Young adults who experience psychological distress may be more likely to reason about the self in negative ways, which in turn fosters and maintains a negative view of the self that in turn contributes to negative emotional experiences. This view is complemented by models of psychopathology that suggest depressed affect is intensified by the interaction between negative cognition (e.g. negative attribution style) and rumination (Ciesla \& Roberts, 2007). That is, young adults who experience psychological distress may ruminate about negative features of the self (through autobiographical reasoning), which in turn amplifies experiences of psychological distress.

Experimental or longitudinal research is needed to ascertain which of these scenarios best accounts for the findings of the current study. Future research could assess whether psychological functioning can be improved by encouraging participants to form positive, in place of negative, self-event connections. Earlier writing studies aimed at encouraging cognitive processing of negative life events have had some success at improving physical well-being and mood symptoms following traumatic experiences with this methodology (see Pennebaker \& Francis, 1996; Smyth, Hockmeyer, \& Tulloch, 2008). It is important to note, however, that age and the developmental stage of participants are 
critical factors to consider in relation to such tasks, as unstructured narrative tasks have been shown to increase psychological distress in children who may lack the regulatory and narrative skills to engage in adaptive reflection (Fivush, Marin, Crawford, Reynolds, \& Brewin, 2007). Furthermore, Honos-Webb, Harrick, Stiles and Park (2000) found some preliminary evidence that expressive writing tasks may increase health centre visits for participants who are re-exposed to traumatic experiences and who are unable to resolve these experiences within the bounds of the study.

While the findings did show that more and more complex reasoning was associated with poorer psychological functioning for young adults who were the least likely to make positive connections, there was no evidence that more and more complex forms of autobiographical reasoning were associated with positive psychological functioning for young adults who were the most likely to reason about the self in positive ways. These findings may suggest that young adults who have a largely positive view of the self experience similar levels of psychological functioning regardless of the extent to which they reflect on these qualities in life story narratives. This interpretation is consistent with earlier work which has shown that insight into the self, self-awareness of thoughts and emotions, but not self-reflection is associated with positive psychological well-being (Harrington \& Loffredo, 2011).

The absence of moderation effects for high points suggests that amount and complexity of autobiographical reasoning in narratives of positive events is not related to an increase/decrease in psychological functioning, even when analysed by individual differences in the propensity to link these experiences to positive or negative characteristics of the self. This finding is consistent with earlier theoretical and empirical work suggesting that deriving meaning from negative events is more consequential for psychological functioning than deriving meaning from positive events (e.g. Lyubomirsky, Sousa \& Dickerhoof, 2006; Pals, 2006; Park, 2008, 2010). For example, Lyubomirsky et al. (2006) suggest that while savouring positive experiences is associated with psychological well-being, evaluating or reasoning about the meaning of positive experiences may force individuals to process the experience in an unhelpful way. There is, however, an alternative methodological explanation for the absence of moderation effects for high points. Almost all (92\%) self-event connections found in narratives of high points were positive, which meant there was little variation between young adults who were the most and least likely to reason about positive/negative characteristics of the self in high points. 


\section{Does autobiographical reasoning valence moderate the relationship between centrality of negative events and psychological functioning?}

In relation to the third aim, findings showed that the tendency to make connections between negative experiences and positive characteristics of the self acted as a buffer against experiences of psychopathology and poor psychological well-being under conditions where these low points were considered central to identity. That is, endorsing low points as central to identity was associated with higher levels of psychopathology and poor psychological well-being, but not for young adults who were most likely to make positive self-event connections in narratives of these experiences. For young adults who were most likely to connect low points to positive characteristics of the self, greater centrality of event ratings were not related to psychopathology and were associated with an increase in psychological well-being.

These findings may help to clarify previously mixed results reported in the literature. While some earlier studies have reported a link between centrality of event ratings for negative or traumatic events and psychological distress (Berntsen \& Rubin, 2006; Boals, 2010), other studies found no evidence for such a relationship (Newby \& Moulds, 2011). No previous work has taken account of the way in which individuals reason about the self in relation to these experiences. In doing so, the current findings suggest that endorsing negative experiences as being a central component of one's identity and life story is not associated with poor psychological functioning if the experience is linked to predominantly positive characteristics or outcomes for the self. These findings are also consistent with previous theoretical and empirical work that has highlighted the psychological benefits of identifying positive outcomes for the self when reflecting on negative life experiences (Davis et al., 1998; King et al., 2000; Lilgendahl \& McAdams, 2011; Pals, 2006; Stein et al., 1997).

Of course, the correlational nature of our analysis again prevents any firm conclusions being made about the mechanisms underlying this moderation effect. One possibility is that focusing on positive outcomes and characteristics of the self in the face of negative experiences is an adaptive strategy that individuals use to help them manage the difficult task of integrating these events into their life story/identity. Alternatively, individuals who function well in the context of major difficult life events may be more likely to reason about positive developments and characteristics of the self in relation to these negative experiences, and endorsing these events as central to identity and the life story poses no threat to this level of functioning. 
In summary, the results of the current study extend previous work by showing that the valence of autobiographical reasoning (linking experiences to positive/negative characteristics of the self) is an important predictor of psychological functioning. Furthermore, individual differences in the propensity to link negative experiences to positive/negative characteristics of the self influences two key relationships: first, the relationship between autobiographical reasoning (amount and complexity) and psychological functioning and second, the relationship between the centrality of negative events and psychological functioning. Future research into the association between life story development and psychological functioning will be enhanced by considering this key individual difference. 


\section{Chapter Five}

\section{Study 1b: Does the valence of autobiographical reasoning found in life story narratives contribute to changes in psychological functioning over time? A pilot analysis}

Study $1 \mathrm{~b}$ was designed to explore, in a preliminary manner, the issue of directionality in the relationship between autobiographical reasoning valence and psychological functioning. One of the major challenges for research investigating links between autobiographical reasoning and psychological functioning is determining where the direction of influence lies within the relationship between these factors (McLean \& Mansfield, 2010). This challenge has been addressed by a small number of studies which have employed longitudinal designs in favour of the cross-sectional designs, and have provided somewhat mixed findings (Pals, 2006; Tavernier \& Willoughby, 2012).

Sutin, Costa, Wethington, and Eaton (2010) asked adults participating in a wider longitudinal study to complete a general measure of psychological distress (measuring anxiety, depression, somatic symptoms and social dysfunction) at two time points ten years apart. At the second time point, participants were asked to report the most stressful life event that had happened in their lives over the past ten years. Participants then reported whether they had learnt a lesson from the experience. Findings showed that learning a lesson from the experience was unrelated to psychological distress at Time 2 (controlling for psychological distress at Time 1). In explaining this unexpected finding, Sutin et al. (2010) suggested that for many participants, the stressful event may not yet have been fully resolved and thus the wider benefit of gaining important life lessons may not have been fully realised. It is also important to note that the measure of lesson learning used in this study required a simple 'yes' or 'no' answer to the question of whether a lesson had been learnt. This indicator is more restricted than, and represents the lowest level of meaning as described in, McLean and Thorne's (2003) original coding scheme. This study did not, therefore, address the question of whether changes in psychological distress over time are associated with more sophisticated forms of meaning.

In contrast to the findings of Sutin et al. (2010), Tavernier and Willoughby (2012) ${ }^{6}$ found that young adults who engaged in autobiographical reasoning (as measured by lesson learning and gaining insight into the self) in relation to turning point events

6 The study by Tavernier and Willoughby (2012) is outlined in more detail in Chapter Two. 
experienced better psychological functioning ${ }^{7}$ after the event than young adults who demonstrated no evidence of autobiographical reasoning following turning point events. Differences in psychological functioning between young adults who engaged in reasoning and those who did not were present after controlling for baseline psychological functioning. Findings also showed that psychological functioning at one time point did not predict whether young adults engaged in autobiographical reasoning following a turning point experience. Two conclusions were drawn from these findings. First, pre-existing levels of psychological functioning do not alter whether or not autobiographical reasoning occurs. Second, the process of autobiographical reasoning itself may account for changes in psychological functioning over time.

The findings of Study 1a showed that the valence of autobiographical reasoning, measured as self-event connections that describe positive and negative characteristics of the self, is a stronger predictor of psychological functioning than the amount of autobiographical reasoning found in life stories constructed by young adults. Specifically, young adults who experienced more negative emotional states such as depression, anxiety and stress were more likely to reason about negative features of the self and less likely to reason about positive features of the self than young adults with better psychological functioning. While these findings provide evidence of a concurrent relationship between autobiographical reasoning valence and psychological functioning, the cross-sectional design of the study prevents any conclusions relating to the directionality of this relationship to be made.

McLean, Pasupathi, and Pals (2007) suggest that at a theoretical level, the valence of autobiographical reasoning may actively contribute to positive psychological functioning. Specifically, connecting past experiences to positive outcomes or characteristics of the self contributes to the development of a life story (and hence identity) that contains within it, positive qualities of the self (e.g. personal strength, increased selfconfidence). This positive view of the self, as expressed through the life story, in turn promotes positive psychological functioning and can moderate the potential negative emotional impact of past, present and future events (McLean et al., 2007).

Just one study to date has investigated the relationship between autobiographical reasoning valence and psychological functioning over time. Pals (2006) defined positive self-transformation as the combination of statements expressing an openness to exploring

\footnotetext{
7 In this study, psychological functioning was defined in terms of reverse-coded symptoms of depression and anxiety, and self-esteem.
} 
the effect of one's experience on the self, and statements describing positive outcomes from negative experiences ${ }^{8}$. Adult women, who, at one time point, constructed life story narratives containing themes of positive self-transformation, were more satisfied with their lives and demonstrated greater self-understanding and emotional maturity almost ten years later. This study did not, however, control for indicators of psychological functioning at time one which meant that themes of positive self-transformation could not be reliably linked to changes in psychological functioning over time.

The current study was designed to address the lack of research examining issues of directionality in the relationship between autobiographical reasoning valence and psychological functioning. Before outlining the specific aims and hypotheses of this study, it is important to note that the sample for this study was drawn from the pool of participants who completed Study 1a. Although all participants were invited to participate, only a small number $(\mathrm{n}=21,21 \%$ of the original sample) completed this study. For this reason, the following study can be considered a pilot only.

The aims of this study were two-fold. The first aim was to examine whether engaging in autobiographical reasoning that describes the self in positive and negative terms remains stable over time or fluctuates according to varying levels of psychological distress. Pasupathi (2012) notes that one of the key limitations of cross-sectional studies on autobiographical reasoning is that we, as researchers, only see a snapshot of this process at one point in time. Rather, Pasupathi (2012) suggests that autobiographical reasoning is a process which likely evolves over time as individuals discuss events with others and gain new perspectives on past events (see also, Pasupathi, 2001). In light of no prior work examining the stability of autobiographical reasoning valence, the question of whether positive and negative autobiographical reasoning would be correlated over time was left open. A second aim of the current study was to explore key relationships between the valence of autobiographical reasoning found in young adults' life story narratives and changes in psychological functioning over time. In accordance with the theoretical notion that engaging in positively/negatively valenced autobiographical reasoning would have a direct impact on psychological functioning, the following hypothesis was made. After controlling for baseline levels of psychological distress, young adults who were more likely to reason about the self in negative ways (i.e. make more negative self-event connections) in life story narratives would experience an increase in psychological distress

8 This study by Pals (2006) is described in more detail in Chapter Two 
over the course of a year, while young adults who were more likely to reason about the self in positive ways (i.e. make more positive self-event connections) in life story narratives would experience a decrease in psychological distress over the course of a year.

\section{Method}

\section{Participants}

Participants from Study 1a were invited to take part in a follow-up study approximately one year following participation in the initial study. Approximately onefifth $(21 \%, \mathrm{n}=21)$ of participants from time one agreed to take part. Table 5.1 compares demographic characteristics for the young adults who participated in Study 1b at Time 2 with the characteristics of the total sample of participants who completed Study 1a.

On average, participants who completed Study $1 \mathrm{~b}$ at Time 2 were just over one year older than the total pool of participants who completed Study 1a one year prior $(M=$ 19.4 years at Time 1 and $\mathrm{M}=20.8$ years at Time $2, p<.05)$. This significant difference is expected, and reflects the 12 month gap that existed between the two time points. The majority of participants who participated in Study $1 \mathrm{~b}$ at Time 2 were female (85\%). This is significantly higher than the $72 \%$ of participants who were female in Study 1a at Time 1 $\left(X^{2}=8.05, p<.05\right)$. There were no significant differences in the ethnicity of participants at Time 1 or Time $2\left(X^{2}=2.33, p>.05\right)$. There was no significant difference in the average number of self-event connections made by participants in Study 1a and Study 1b $(t(31)=1.16, p>.05)$ nor was there a significant difference in DASS scores between participants in Study 1a and Study 1b $(t(32)=.303, p>.05)$. Participants received vouchers to a local cinema as a token of appreciation for their participation.

\section{Overview of Procedure}

Participants were invited to take part in the follow-up study via email and those who agreed to take part completed the follow-up session through an online survey created in Qualtrics (Qualtrics Labs Inc., Provo, UT). Participants first read a summary of the nature and purpose of the study and provided consent. Following this, they completed the same autobiographical narrative task as completed at time one and also completed the Depression Anxiety Stress Scale (DASS) as a measure of psychological distress. 
Table 5.1

Differences in participant characteristics and autobiographical reasoning measures at

Time 1 and Time 2 (one year later)

\begin{tabular}{lll}
\hline Measure & Time 1 & Time 2 \\
\hline Age & $\mathrm{M}=19.4$ yrs (SD =2.1 yrs $)$ & $\mathrm{M}=20.8$ yrs (SD = 2.6 yrs) \\
Gender & $72 \%$ female & $85 \%$ female \\
Ethnicity & $70 \%$ NZ European & $70 \%$ NZ European \\
$\begin{array}{l}\text { Average number of } \\
\text { self-event connections }\end{array}$ & $\mathrm{M}=1.55(\mathrm{SD}=.83)$ & $\mathrm{M}=1.33(\mathrm{SD}=.75)$ \\
Total DASS scores & $\mathrm{M}=32.94(\mathrm{SD}=30.36)$ & $\mathrm{M}=30.95(\mathrm{SD}=26.71)$ \\
\hline
\end{tabular}

\section{Tasks and measures}

The tasks and measures used at time are described in more detail in Chapter Four.

Psychological distress. Depression Anxiety Stress Scale (DASS; Lovibond \&

Lovibond, 1995).This self-report questionnaire measures three related negative emotional states: depression, anxiety and stress, each on a 14-item subscale. Participants rate on a four-point scale how much each symptom applied to them over the past week $(0=\operatorname{Did}$ not apply to me at all, $3=$ Applied to me very much, or most of the time). Consistent with previous research (Crawford \& Henry, 2003), a total DASS score was calculated by summing each of the subscales. Internal consistency for the DASS total score within the current sample of 21 participants was .97.

Autobiographical narrative task. Life story narratives were attained through an autobiographical narrative task modified from McAdams' guided autobiography http://www.sesp.northwestern.edu/foley/instruments/guided/. Participants were asked to provide written narratives of two high points, "moments or episodes in a person's life in which he or she feels a sense of happiness, joy, excitement, contentment or some other positive emotion" and two low points, "moments or episodes in a person's life in which he or she feels extremely negative emotions such as despair, disillusionment, guilt, shame etc." from their life story. 


\section{Narrative coding}

Self-event connections. Following the procedure outline in Study 1a (Chapter Four), self-event connections were coded as any statements where participants explicitly linked an aspect of the narrated event to their sense of self. Fifty-two percent of narratives were coded by an independent coder. Consistent with the approach taken in Study 1a, the number of self-event connections was calculated on a per narrative basis and inter-rater agreement for the total number of connections was kappa .86. Each self-event connection was coded for valence $(0=$ neutral connection; $1=$ positive connection; $2=$ negative connection; $3=$ mixed positive and negative connection). As per Study 1a, self-event connections that were identified by both coders $(91 \%$ of connections identified by the first coder) were compared for valence. Inter-rater agreement for the valence of self-event connections was kappa .80 .

\section{Results}

The following results should be treated as indicative only due to the small number of participants included in the analysis. To examine the hypothesis that young adults' tendency to engage in autobiographical reasoning that describes the self in positive/negative ways would remain stable over time, Pearson correlations compared relationships between the number of negative self-event connections and the number of positive self-event connections found at the two time points. The number of negative selfevent connections made at time one and time two were significantly correlated $(r=.48, p<$ $.05)$. The number of positive self-event connections made at time one and time two were not significantly correlated $(r=.34, p>.05)$. Symptoms of psychological distress (DASS scores) at time one and time two were significantly correlated $(r=.54, p<.05)$.

To further examine the stability of the relationship between the number of negative self-event connections made at time one and time two, a partial correlation was conducted between the number of negative self-event connections made at time one and time two, controlling for change in DASS scores. Controlling for change in DASS scores between the two time points, the relationship between number of negative self-event connections remained significant $(r=.51, p<.05)$.

To examine the hypothesis that the valence of self-event connections found in life story narratives would predict changes in psychological distress, hierarchical regression examined whether the number of positive and negative self-event connections made at time one predicted change in DASS scores approximately one year later. DASS scores at time 1 were entered in the first step in order to control for participants' initial experiences 
of negative emotional states. The average number of positive and negative self-event connections at time 1 were entered in the second step to examine the extent to which the valence of autobiographical reasoning explained unique variance in the change of negative emotional states over time.

Regression analyses demonstrated that just one predictor (DASS scores at time one) explained $33 \%$ of the variance in change in DASS scores over time $\left(R^{2}=.23, F(3,17)\right.$ $=8.53, p<.05)$. Participants who scored higher on the DASS at time one experienced less change (more stability) in DASS scores over time. Neither the number of positive or number of negative self-event connections found in life story narratives of low and high points (combined) significantly predicted change in DASS scores over time $(\beta s=-.12$ to $.12, p s<.05)$.

\section{Discussion}

Before discussing the findings of the current study, it is important to remember that the results should be treated as indicative only. The small number of participants in the study prevents any firm conclusions being made from any of the findings. The extent to which the sample in the current sample is representative of the sample at Time 1 is also questionable. Participants in this study were more likely to be female and slightly older than participants overall at Time 1. Prior work has shown that females tend to construct longer narratives and may include more expressive and emotional content in their narratives than do males (Bauer, Stennes, \& Haight, 2003; Fivush, Brotman, Buckner, \& Goodman, 2000).

The aims of this study were two-fold. The first aim was to examine stability in the valence of autobiographical reasoning found in life story narratives constructed by young adults over the period of one year. The second aim was to examine the relationships between autobiographical reasoning valence and psychological functioning using a longitudinal design. In using this methodology, the current study aimed to investigate whether constructing narratives containing positively and negatively valenced autobiographical reasoning would predict future psychological functioning.

With respect to the first aim, findings showed that the number of negative, but not the number of positive, self-event connections young adults made in narratives of low and high points from the life story were significantly related at the two time points. Thus, young adults who made more negative connections in narratives of life story high and low points at time one were also likely to make more negative connections in narratives of low and high points one year later. This previously untested association suggests that making 
negative self-event connections may be a relatively enduring pattern that fluctuates very little over time. Further analysis showed that the relationship between negative self-event connections remained significant after controlling for changes in negative emotional states experienced between the two time points. This finding suggests that the extent to which young adults' reasoned about the self in negative ways remained relatively stable in the context of fluctuations in negative emotional states. This finding challenges the notion that fluctuations in autobiographical reasoning valence over time are related to changes in distress levels.

The lack of a significant relationship between positive self-event connections made at time one and time two suggests that unlike making negative self-event connections, the extent to which young adults reason about the self in positive ways is more prone to fluctuate over time. The consistency in making negative but not positive connections over time is interesting and requires explanation. One possible explanation may be drawn from theory and research pertaining to self-schemas. Self-schemas are mental representations of the self that contain information pertaining to an individual's beliefs about the type of person they are, characteristic behavioural patterns and internal experiences such as thoughts, emotions and physiological reactions (Beck, 1995; Welburn, Coristine, Dagg, Pontefract, \& Jordan, 2002).

Malle and Horowitz (1995) have argued that negative self-schemata are more tightly organised and consistently activated than positive self-schema. In one study, young adults judged whether positive and negative self-descriptors 'go together' (i.e. are often expressed together), are 'opposites' (i.e. each time one trait is expressed the other is not expressed) or 'independent (i.e. unrelated). Findings showed that negative self-descriptors were more commonly judged as 'going together' than were positive self-descriptors, thus suggesting that negative self-relevant information is organised more tightly in schematic form than is positive self-relevant information. Further to this, Malle and Horowitz (1995) also found that individuals who were more likely to endorse negative self-descriptors did so more consistently across various measures than did individuals who were more likely to endorse positive self-descriptors. These findings have two main implications. First, negative self-relevant information may be more likely than positive self-relevant information to be perceived as highly inter-connected. Second, this highly inter-connected negative self-schema is more likely to be activated in response to different cues than the corresponding positive self-schema. 
What might these results mean for consistency in positive and negative self-event connections over time? If negative self-event connections are a part of an individual's negative self-schema, then following the findings of Malle and Horowitz (1995) a greater consistency in negative self-event connections over time would be expected. That is, negative self-relevant information may be more likely to be activated by a life story task and be more consistently applied across a range of events than positive self-relevant information. The link between positive/negative self-event connections and self-schema has not yet been tested and further research is needed to establish whether this is a viable explanation for the findings.

With respect to the second aim, findings showed that neither the number of negative nor the number of positive self-event connections made at time one significantly accounted for the change in participants' experiences of negative emotional states over time. Although these findings must be considered in the context of the limitations raised earlier, this finding suggests that the extent to which young adults reason about the positive or negative implications of past experiences for the self in the context of life story narratives does not actively enhance or diminish experiences of negative emotional states. This finding does not support the theoretical assertions made by McLean, Pasupathi, and Pals (2007), that making more positive self-event connections and reducing the number of self-event connections made in life story narratives over time can result in positive changes to psychological functioning. Instead, the findings suggest that the relationship between autobiographical reasoning valence and psychological functioning remains may simply be a concurrent relationship. Thus autobiographical reasoning valence may reflect coexisting levels of, but does not actively impact, psychological functioning.

An obvious limitation of the current study is the small sample size $(n=21)$. This small sample size means that the analyses performed must be regarded as indicative only and require validation through future work with higher sample sizes. Additionally, to reduce demands on participants, only one measure of psychological functioning was used; the Depression Anxiety Stress Scale. The findings presented in Chapter Four show that positive self-event connections were associated with a broader measure of psychological functioning that included qualities such as autonomy, personal growth, mastery and positive relationships with others. The possibility that an increase in positive self-event connections over time would be associated with increases in more global measures of psychological well-being cannot be discounted and requires further investigation. 
An interesting area of investigation for future research is consistency in the valence of autobiographical reasoning found in relation to the same event over time. In the current study, participants were not specifically asked to narrate the same events as narrated at Time 1. points. For most participants, the events narrated at time two were not the same events narrated at time one. Pasupathi (2012) suggests that individuals process their life events over a significant amount of time, and the interpretations and understanding of these events is likely to change as individuals gain new experiences and perspectives on these events. This is proposed to be especially true if the event is discussed with others and thus open to the influences of social construction (Pasupathi, 2001). Although untested, this notion is also likely to apply to the extent to which individuals' reason about the self in positive and negative ways in relation to key events. Consider the following example provided by one of the participants in the current study:

\begin{abstract}
When I was 12 years old ... there was a boy that I liked in my class and I thought he might like me too. I sent my friends over to ask him out for me. I was turned down much to my distress. I had found it very hard to get up the courage to even mention that I liked a boy let alone have him reject me via my friends ... I consider that to be a pivotal moment in my life. I was teased in primary school for being overweight and I felt that his rejection was a reflection on my physical appearance ... For my whole life after that point I am very guarded about my feelings. This has been detrimental in a couple of relationships.
\end{abstract}

This moment has obviously been influential in the young adults life and has had a lasting impact on her ability to share her emotional experiences with others. It is possible, however, that this view of herself could change under certain conditions. For example, entering into a supportive relationship where she is encouraged to share her feelings and is accepted for who she is may lead her to feel more positively about herself. Under these conditions, we might expect this event that occurred many years ago to be interpreted in a different light. That is, instead of the event being framed as a reflection of her own shortcomings, the young adult may identify that the boy may have turned her down for a number of reasons, none of which reflect judgements on her physical appearance. Tracking changes in autobiographical reasoning valence over time is an interesting question for future research. Furthermore, it would be interesting to examine whether 
shifts from negative to more positive or neutral statements about the self are associated with shifts in psychological functioning, and if so, whether these shifts are causally related or related to a third variable (e.g. entering a supportive relationship).

In summary, this pilot study raised two interesting findings. First, young adults' tendency to make negative self-event connections in life story narratives remains stable over time, possibly reflecting a negative self-schema that is activated when completing a lie story narrative task. Second, young adults' tendency to make positive or negative selfevent connections at one time point did not predict changes in psychological functioning over time. This latter finding suggests that autobiographical reasoning may not have a direct effect on psychological functioning, which was hypothesised. The findings of the current pilot highlight a need to examine these questions with larger, more reliable sample sizes. 


\section{Chapter Six}

\section{Study 2: An investigation into the relationships between autobiographical reasoning, cognitive response styles, meaning in life and psychological functioning}

The findings presented in Chapter Four reveal evidence of a relationship between psychological functioning and the valence of autobiographical reasoning found in the life story narratives of young adults. That is, young adults who made more positive self-event connections in narratives of high and low points from their life story experienced better psychological well-being and fewer symptoms of psychological distress (depression, anxiety and stress) than young adults who made fewer positive self-event connections. Conversely, young adults who made more negative self-event connections experienced more symptoms of psychological distress and poorer well-being than young adults who made fewer negative connections. These findings supplement a broader body of research which has shown that linking negative past experiences to positive features or developments in the self through autobiographical reasoning is associated with positive psychological functioning (King et al., 2000; Lilgendahl \& McAdams, 2011; McAdams, et al., 2001; Pals, 2006). A number of key questions remain, however, regarding why this relationship exists. The aim of this Chapter is to build on the knowledge gained in Chapter Four by exploring key factors that may be associated with the tendency to engage in positive and negative autobiographical reasoning in young adulthood.

McLean and Mansfield (2010) note that the relationship between autobiographical reasoning and psychological functioning may be explained by an additional, or third, variables. There are a great number of factors that may predict, or interact with, autobiographical reasoning valence. This Chapter examined associations between autobiographical reasoning valence and a number of key factors derived from cognitive models of psychological distress and meaning-based models of psychological well-being. From the cognitive literature, these factors include explanatory style, rumination and cognitive reappraisal. From the meaning-based literature, the key factor examined was meaning in life. The current study had two main aims. First, to investigate key relationships between the valence of autobiographical reasoning found in young adults' life story narratives and each of these cognitive and meaning-based factors. These factors were chosen on the basis of their theoretical overlap to the autobiographical reasoning construct. The second aim was to investigate whether, and how, these factors interact with 
autobiographical reasoning valence to predict psychological functioning. Consistent with Ryff and Singer (1996), psychological functioning in the current study was again defined in terms of psychological distress and psychological well-being. Specific predictions, which will now be discussed, were made in relation to the cognitive and meaning-based factors and psychological distress and well-being.

\section{Outline of cognitive response styles}

Cognitive theories of psychological distress suggest that the manner in which individuals process information about events and the self can account for the development and maintenance of emotional disorders such as depression and anxiety (Beck, 1995). These theories propose that dysfunctional schema, cognitive frameworks of the self and the world, influence how one's experiences are interpreted (Welburn, Coristine, Dagg, Pontefract, \& Jordan, 2002). In certain contexts (e.g. stressful or negative life events), dysfunctional schema activate maladaptive thoughts and beliefs about the experience, the self and the world which in turn leads to experiences of emotional distress (Riskind \& Alloy, 2006). In the case of depression, schema are generally assumed to reflect an overly negative view of the world and the self, while in the case of anxiety, schema reflect a biased and inflated view of the world and environmental stimuli as dangerous and a relative inability to cope with these situations (Beck \& Clark, 1997; Laidlaw \& Davidson, 2001; Segal, Hood, Shaw \& Higgins, 1988).

Certain cognitive vulnerability factors, styles of thinking about and interpreting one's experience, act in conjunction with dysfunctional schema to increase the likelihood of developing emotional disorders (Alloy et al., 1999; Riskind \& Alloy, 2006). Extant theoretical and empirical evidence suggests that two such factors, explanatory style and rumination, are among the strongest and most reliable predictors of the development and maintenance of emotional distress. These two factors are conceptualised as 'transdiagnostic' in that they underlie multiple indicators of psychological distress. For example, each factor is proposed to play a key role in maintaining the highly comorbid conditions of depression and anxiety (McLaughlin \& Nolen-Hoeksema, 2011; Watkins, 2009). Each of these factors will now be discussed in more detail.

\section{Explanatory style}

Explanatory or attribution style, first described by Abramson, Seligman and Teasdale (1978), refers to characteristic ways of accounting for, or attributing cause to, positive and negative life events. Explanatory statements vary along dimensions of internality, i.e. 'the event was caused by me' versus externality 'the event was caused by 
someone/something else', can be stable, i.e. 'this cause will be around forever' or unstable 'this cause is transitory', and can be global, i.e. 'this cause will be present in all situations' or specific 'this cause was just present in this situation' (Morris, 2007). A negative explanatory style is defined as the tendency to attribute the cause of negative life events to internal, stable and global characteristics of the self (Abramson et al., 1978)

Adopting a negative explanatory style is considered a risk factor for the development of depression and anxiety following negative or stressful life events (Abramson et al., 1978; Alloy et al., 1999; Peterson \& Seligman, 1984; Reardon \& Williams, 2007). Findings support this theoretical notion, and have found that adopting a negative explanatory style is associated with a greater number and recurrence of depressive episodes, trauma symptoms following traumatic experiences in both adults and children and negative affect and anxiety in college students (Luten, Ralph, \& Mineka, 1997; Mongrain \& Blackburn, 2005; Palker-Corell \& Marcus, 2004; Runyon \& Kenny, 2002). Adopting a negative explanatory style has also been shown to predate, and predict, future depressive symptoms (Sanjuan \& Magallares, 2009). Although the link between psychological distress and the explanatory style used to explain positive events is more tenuous, there is some evidence that attributing positive experiences to internal, stable and global causes predicts decreases in hopelessness and recovery from depression (Johnson, Han, Douglas, Johannet \& Russell, 1998; Needles \& Abramson, 1990) whereas attributing positive events to external, unstable and specific causes is associated with higher levels of depressive symptomatology (Fresco, Alloy, \& Reilly-Harrington, 2006).

\section{Rumination}

Rumination is defined as a response to psychological distress that is characterised by repetitive thoughts about the causes and consequences of the symptoms of psychological distress (Nolen-Hoeksema, 1991). Like negative explanatory style, rumination has been conceptualised as a cognitive vulnerability factor for experiences of psychological distress. Rumination tends to be perseverative in nature. That is, individuals tend to focus solely on their experience of distress and do so in a passive manner rather than taking an active problem solving approach to their distressed state (Nolen-Hoeksema, Wisco, \& Lyubomirsky, 2008). Thus while rumination maintains focus on distress symptoms, it does not lead to adaptive coping strategies or problem solving techniques that can alleviate distress. Consistent with this notion, rumination has been shown to exacerbate the relationship between stressful life events and emotional distress (depression, anxiety and stress) in young adults (Marks, Sobanski, \& Hine, 2010), 
has been found to predict both depression and anxiety in a sample of adults with diagnosed mood disorders (Watkins, 2009) and has been associated with an increase in negative affect and depressive symptoms (McLaughlin, Borkovec, \& Sibrava, 2007). Furthermore, rumination induction studies have shown that inducing a ruminative response style to negative life events causes transient increases in negative, and decreases in positive, affect (Grisham, Flower, Williams, \& Moulds, 2011).

\section{Cognitive reappraisal strategies}

In contrast to the maladaptive cognitive processes of negative explanatory style and rumination, individuals can also use a number of adaptive cognitive processes to minimise experiences of emotional distress. Cognitive reappraisal, defined as an emotion regulation strategy which involves changing the meaning of events in order to modify its emotional impact, is one such factor (Gross, 1998; Gross \& John, 2003). From a theoretical perspective, cognitive reappraisal does not actively prevent the development and maintenance of emotional distress, but is an active strategy individuals can use to minimise the experience of negative emotions in relation to unwanted events (Gross \& John, 2003). In support of this view, individuals who characteristically use reappraisal strategies experience lower levels of depression and negative emotion, are less likely to appraise themselves, their lives and their future in a negative way, and experience higher levels of positive emotion, satisfaction with life, self-esteem, psychological well-being than those less likely to use reappraisal strategies (Gross \& John, 2003; Mauss, Cook, Cheng, \& Gross, 2007; Nezlek \& Kuppens, 2008).

\section{Similarities between cognitive response styles and autobiographical reasoning}

The three cognitive factors described above (explanatory style, rumination and cognitive reappraisal), share key conceptual similarities with the process of autobiographical reasoning. According to key proponents of cognitive models of psychological distress (Beck, 1995; Riskind \& Alloy, 2006), negative emotional states such as depression and anxiety are associated with the way individuals understand and interpret their experiences. Autobiographical reasoning also refers to the process of interpreting and processing information about the self and one's experiences. Despite this conceptual similarity, research has not yet investigated the relationships between cognitive response factors and the valence of autobiographical reasoning found in narratives of life story events.

A small number of studies, however, have investigated whether key cognitive response styles can be observed within the life story. Habermas, Ott, Schubert, Schneider 
and Pate (2008) coded life story narratives of depressed and non-depressed individuals for indicators of negative cognitive biases and explanatory style. Depressed individuals were more likely to draw on negative life events to construct their life story and adopt a negative explanatory style when narrating their experiences than were non-depressed individuals. While these findings suggest that expressions of a negative explanatory style can be found in life story narratives, this study did not specifically investigate differences in autobiographical reasoning between the depressed and non-depressed participants.

An earlier study examined associations between negative explanatory style and McAdams et al's (2001) conceptualisation of contamination sequences (Adler, Kissel, \& McAdams, 2006). Adler et al. (2006) coded adults' life story narratives for expressions of a negative explanatory style using the Content Analysis of Verbatim Explanations (CAVE; Peterson, Schulman, Castellon, \& Seligman, 1992). The CAVE is a common tool used to assess explanatory style in narratives of personal experiences and provides a reliable alternative to other measurement tools for explanatory style which rely on hypothetical events (Peterson, 1991). Life story narratives were also coded for contamination sequences, descriptions of positive events or states that are followed by a negative ending or outcome and participants completed measures of depression, self-esteem and life satisfaction. As expected, symptoms of depression and lower levels of life satisfaction were associated with greater expression of a negative explanatory style and more contamination sequences. While negative explanatory style was unrelated to contamination sequences, both of these variables (explanatory style and contamination sequences) explained unique variance in depressive symptoms. In explaining the nonsignificant relationship between negative explanatory style and contamination sequences, the authors note that these two measures represent different 'cognitive categories'. That is, explanatory style reflects an individual's explanation of events, while contamination sequences describe a pattern of affective experiences. Neither of these studies specifically examined the relationship between autobiographical valence and explanatory style, however. Similarly, no prior work has investigated relationships between autobiographical reasoning valence and the remaining two factors: rumination and cognitive reappraisal.

Part 1a: Relationship between positive/negative self-event connections and cognitive response styles

The first area of investigation in the current study explored key relationships between the number of positive and negative self-event connections found in life story narratives and each of the three cognitive response factors: explanatory style, rumination 
and cognitive reappraisal. To investigate these relationships, young adults 18 to 28 years of age were asked to write about a high point, low point and turning point from their life story and to complete measures assessing explanatory style, rumination and their use of cognitive reappraisal strategies. In light of the limited extant literature, three tentative hypotheses were made.

First, I hypothesised that the number of negative self-event connections found in narratives of life story low points would be positively correlated with negative explanatory style and the number of positive self-event connections found in narratives of life story high points would be positively associated with positive explanatory style. As turning point experiences can be negative or positive, the question of whether explanatory style would relate to positive/negative self-event connections found in narratives of these events was left open.

Second, I hypothesised that rumination would be correlated with the number of negative self-event connections found in narratives of all event types. This relationship was expected to occur across all event types as rumination is conceptualised as a general tendency to think about the causes of one's emotional distress, and is not tied to certain types of events. I note, however, that negative events are likely to cause more distress and thus the relationship between rumination and negative autobiographical reasoning may be stronger in relation to narratives of low points.

Third, I hypothesised that cognitive reappraisal strategies would be correlated with the number of positive self-event connections found in narratives of life story low points and unrelated to the number of positive self-event connections in narratives of high points. This hypothesis was set as cognitive reappraisal strategies are only expected to be activated in the context of events that elicit negative emotions. Again, the question of whether reappraisal strategies would be associated with positive self-event connections found in narratives of turning points was left open.

\section{Part 1b: Relationships between positive/negative self-event connections, cognitive response styles and psychological distress}

The second set of hypotheses addressed the inter-relationships between individual differences in the use of the three cognitive response factors (explanatory style, rumination and cognitive reappraisal), positive/negative self-event connections and psychological distress. These hypotheses were arranged in three steps. First, before examining some of the more complex interactions between these variables, I expected to replicate the findings presented in Chapter Four, that increased levels of psychological distress would be 
associated with a higher number of negative, and a lower number of positive, self-event connections in life story narratives overall. The second hypothesis addressed the question of whether the narrative variables (number of positive/negative self-event connections) would explain unique variance in symptoms of distress over and above that explained by the cognitive factors (explanatory style, rumination and cognitive reappraisal). In light of previous work highlighting the strong associations between cognitive response factors and psychological functioning, and the assumption that the three cognitive factors and autobiographical reasoning comprise similar processes, I predicted that neither positive nor negative self-event connections would explain unique variance in symptoms of psychological distress beyond what is accounted for by the three cognitive factors.

Third, hypotheses were made to examine possible interactions between positive/negative self-event connections, the cognitive response styles and psychological distress. Specifically, I was interested in whether the three cognitive factors would mediate and/or moderate the relationships between positive/negative self-event connections and psychological distress. A mediating variable is one that explains or accounts for the relationship between two variables. Mediation analysis utilises path diagrams to posit a potential causal chain between three variables and in doing so, attempts to answer the question of why the independent variable to dependent variable relationship exists (Baron \& Kenny, 1986). A moderating variable, on the other hand, is one that influences the strength of the relationship between an independent and dependent variable. Moderation analysis differs from mediation analysis in that it seeks to examine whether the relationship between the independent and dependent variables differs in the context of varying levels of a third variable (Baron \& Kenny, 1986). From a theoretical perspective, variables can act as both a mediator and a moderator (Preacher, Rucker, \& Hayes, 2007) so three sets of hypotheses were made.

The first set of hypotheses explored the question of whether adopting a negative explanatory style would act as a mediator and/or a moderator in the relationship between negative self-event connections and psychological distress. Psychological distress is associated with a negative representation of the self, which is easily accessed when making attributions about the cause of negative events (Bentall, Kinderman \& Bowen-Jones, 1999). There is also some evidence that negative representations of the self are easily accessed during the construction of life story narratives for individuals experiencing a high level of psychological distress (Habermas et al., 2008). In light of these findings, I predicted that the number of negative self-event connections found in narratives of low and 
turning points would interact with greater use of negative explanatory style to predict higher levels of psychological distress (moderation hypothesis). The decision to include autobiographical reasoning that occurred in narratives of turning points (in addition to low points) reflects the fact that these events are frequently negative in nature (see Tavernier \& Willoughby, 2012) and thus are the types of events that a negative explanatory style would be expected to appear in relation to. I also predicted that young adults who made more negative self-event connections in narratives of low and turning points would be more likely to adopt a negative explanatory style, which in turn would be associated with an increase in psychological distress (mediation hypothesis).

The second set of hypotheses examined whether rumination would mediate or moderate the relationship between negative self-event connections and psychological distress. Rumination is a process by which individuals think about the cause of psychological distress in a perseverative and repetitive manner. Greater recall of negative self-related information has been associated with post-event rumination in young adults (Mellings \& Alden, 2000), although the direction of influence has not been well established. In light of these findings, I predicted that young adults who made more negative self-event connections in narratives of low and turning points would be more likely to engage in a pattern of ruminative thinking, which in turn would be related to psychological distress (mediation effect). As, from a theoretical perspective, rumination is more likely to occur in the context of distress caused by negative or stressful life events, these effects were examined in relation to autobiographical reasoning found in narratives of low points and turning points combined. I also predicted that making negative selfevent connections in narratives of low and turning points would interact with rumination to predict higher levels of psychological distress. That is, the relationship between making negative self-event connections and psychological distress would be stronger for individuals who tend to ruminate about the cause of their distress (moderation effect).

The third set of hypotheses examined whether cognitive reappraisal strategies would mediate or moderate the relationship between positive self-event connections and psychological distress. Cognitive reappraisal strategies result in the attachment of positive meaning to a negative emotion-eliciting event. Such appraisals and the identification of positive outcomes from negative experiences have been associated with better psychological functioning in the years following the event (Davis, Nolen-Hoeksema, \& Larson, 1998; Stein, Folkman, Trabasso \& Christopher-Richards, 1997). It is therefore possible that young adults who report greater use of general reappraisal strategies to 
decrease negative emotional experiences would be more likely to link negative experiences to positive characteristics of the self in narratives of these experiences. Thus I predicted that young adults who made more positive self-event connections in narratives of low and turning points would be more likely to report greater use of cognitive reappraisal strategies, which in turn would be related to lower levels of psychological distress (mediation effect). As, from a theoretical perspective, cognitive reappraisal strategies are only activated in the context of negative or stressful life events, I examined these effects in relation to autobiographical reasoning in the context of low points and turning points combined. I also predicted that making positive self-event connections in narratives of low and turning points would interact with cognitive reappraisal strategies to predict lower levels of psychological distress. That is, the relationship between making positive selfevent connections and lower levels of psychological distress would be stronger for individuals who tend to engage in cognitive reappraisal strategies more generally (moderation effect).

\section{Introducing the concept of meaning in life}

Contemporary well-being theorists have argued that positive psychological functioning consists of the absence of psychological distress and the presence of psychological well-being (Cowen, 1991; Ryff \& Singer, 1996). A key motivation for well-being theorists is the detection of psychological processes and outcomes that contribute to 'optimal' psychological functioning. In addition to the absence of negative psychological states, the notion of psychological well-being is defined in terms of constructs which engender a positive view of the self and one's life.

Ryan and Deci (2001) suggest that psychological well-being consists of both hedonic and eudaimonic well-being. Where hedonic well-being is defined in terms of subjective well-being, and encompasses notions of happiness, positive and negative affect and a sense of satisfaction with one's life, eudaimonic well-being is defined in terms of leading a life that is fulfilling and meaningful (Ryan \& Deci, 2001). Eudaimonic wellbeing has been operationalised in a number of ways, including individuals' subjective appraisals of meaning in life (Steger, Frazier, Oishi, \& Kaler, 2006) and in terms of the proposed components of a meaningful life. These include self-acceptance, positive relations with others, autonomy, environmental mastery, purpose in life and personal growth (Ryff \& Keyes, 1995). Kashdan, Biwas-Diener and King (2008), however, argue that there is a large degree of overlap between hedonic and eudaimonic well-being and thus they should not be conceptualised as independent constructs. Nonetheless, both Ryan 
and Deci (2001) and Kashdan et al. (2008) agree that having a sense of meaning in one's life is an important component of psychological well-being.

Meaning in life has been linked to a number of positive psychological outcomes. Zika and Chamberlain (1992) asked female adults and male and female older adults to indicate their level of satisfaction with their lives and complete a measure of general mental health which included aspects of positive well-being (e.g. cheerfulness, interest in and enjoyment of life) and aspects of poor mental health (e.g. depression and anxiety). Meaning in life was measured in terms of participants' subjective assessments of the extent to which they experience purpose in their lives, the degree to which participants had major life goals and the extent to which participants felt these goals were being achieved. Participants who reported greater meaning in life were more satisfied with their lives, experienced better psychological well-being and less psychological distress than participants who reported less meaning in their lives.

\section{Relationship between autobiographical reasoning valence and meaning in life}

From a theoretical perspective, autobiographical reasoning is associated with the generation of meaning in one's life. Baumeister and colleagues propose that people are inherently motivated to find meaning in their lives and that this motivation drives people to construct narratives of their experiences (Baumeister \& Newman, 1994; Baumeister \& Wilson, 1996). In the context of constructing life story narratives, McLean and Thorne (2003) define the achievement of meaning as "what one gleans from, learns, or understands from the event" (p. 636). In defining meaning in this way, McLean and Thorne (2003) suggest that the process of 'meaning-making' (i.e. that is achieved through autobiographical reasoning) involves considering the wider implications of events for the self and for one's life (see also McLean \& Pratt, 2006; Singer, 2004).

The extent to which the construction of meaning in relation to certain key events generalises to a broader sense of meaning in one's life has not yet been examined. The current study aimed to address this gap in the literature by examining relationships between self-event connections made in life story narratives and young adults' perceptions regarding meaning in their lives. From a theoretical perspective it is possible that the process of making positive, but not negative, self-event connections may be associated with a greater sense that one's life has meaning. As noted earlier, Ryff and Keyes' (1995) scales of psychological well-being measure six components that have been conceptually linked to the establishment of a meaningful life. These include self-acceptance, positive relations with others, autonomy, environmental mastery, purpose in life and personal 
growth. The content of positive self-event connections can readily be categorised into similar themes. For example, McLean and Fournier (2008) found that a significant proportion of self-event connections made in young and older adults' narratives of selfdefining memories described personal growth, personal values, which may correlate with purpose in life, and dispositions, which may correlate with self-acceptance, autonomy and environmental mastery.

Examples of positive self-event connections from Study 2 presented in Chapter Four also highlight the overlap between positive self-event connections and the proposed components of meaning in life. These include:

Themes of self-acceptance:

“A high point for me was when I 'came out' ... This is important to my life, because although I don't let my sexuality wholly dictate who I am it is an integral part of my identity"

Themes of environmental mastery:

"When I was 17 I went on an outdoors adventure camp ... At the end of the course I remember feeling a sense of accomplishment. I felt that I really could do whatever I wanted in life If I just tried and set my mind to it ... It showed me I can push myself and succeed",

Themes of purpose in life:

"One high point in my life was the first gig that my band played back when I was 17 ... I am extremely passionate about music, and performing is one of my most enjoyable things ... my confidence and desire to go further in the music business has increased",

And themes of personal growth:

"A low point for me was when I suffered from panic attacks and mild depression at age 16.... I believe this event was instrumental in my growth as a person ... I appreciate the good times even more".

Unlike positive self-event connections which seem to correlate well with the components of meaning in life, negative self-event connections may demonstrate the opposite relationship. That is, negative self-event connections may be associated with a 
relative absence of meaning in life. For example, the following negative self-event connection is likely to be negatively associated with a number of Ryff's indicators, particularly self-acceptance, positive relations with others, and environmental mastery:

"I was bullied by a friend at school ... This has made me more paranoid, and I find it incredibly difficult to trust people, even my closest friends"

\section{Part 2a: Relationship between autobiographical reasoning valence and meaning in life}

The second area of investigation in the current study explored relationships between the number of positive and negative self-event connections found in life story narratives and young adults' subjective appraisals of meaning in their lives. In light of the theoretical assumption that autobiographical reasoning is involved in the construction of meaning from our experiences (McLean \& Thorne, 2003), and this meaning is in turn associated with a broader sense of meaning in life (Singer, 2004), the following hypotheses were made. First, I predicted that young adults who reported greater meaning in their lives would make more positive self-event connections in narratives of all event types (high, low and turning points) than young adults who reported less meaning in life. I predicted that the number of negative self-event connections found in life story narratives, on the other hand, would be inversely associated with meaning in life ratings.

\section{Part 2b: Relationships between autobiographical reasoning valence, meaning in life and psychological well-being}

A second set of hypotheses addressed the inter-relationships between positive/negative self-event connections, meaning in life and psychological well-being. Young adults' satisfaction with life was chosen as the measure of psychological well-being in the current study. Satisfaction with life is conceptualised as individuals' subjective judgements about how well their life compares to their own notion of a quality life (Pavot \& Diener, 1993). Satisfaction with life was chosen as a measure of psychological wellbeing over Ryff's scales of Psychological Well-Being used in Study 1a as these latter scales overlap considerably with the construct of meaning in life (Ryan \& Deci, 2001).

Before examining some of the more complex interactions between positive/negative self-event connections, meaning in life and psychological well-being, the question of whether the narrative variables (number of positive/negative self-event connections) explained unique variance in life satisfaction over and above that explained 
by meaning in life was addressed. I predicted that neither positive nor negative self-event connections would explain unique variance in satisfaction with life beyond what is accounted for by meaning in life. This hypothesis was made in light of prior work highlighting a strong association between life satisfaction and meaning in life (Steger \& Kashdan, 2007; Steger, Oishi, \& Kesebir, 2011) and the theoretical notion that meaning in life is developed through autobiographical reasoning.

To explore possible interactions between positive/negative self-event connections, I then addressed the question of whether meaning in life would both mediate and/or moderate the relationships between positive/negative self-event connections and life satisfaction. In light of limited extant findings regarding the relationships between these key variables, the following predictions were made. First, I predicted that young adults who made more positive self-event connections in life story narratives would be more likely to report meaning in their lives, which in turn would be associated with an increase in life satisfaction (mediation hypothesis). Second, I predicted that the number of positive self-event connections in life story narratives and young adults' meaning in life ratings would interact to predict higher levels of satisfaction with life. That is, the relationship the number of positive self-event connections and life satisfaction would be the strongest for individuals who report meaning in their lives (moderation hypothesis). The opposite relationships were predicted for negative self-event connections.

\section{Summary of the current study}

In summary, the current study had two main areas of investigation. The first area examined 1) whether autobiographical reasoning valence (as measured by positive and negative self-event connections) were associated with each of the three cognitive response factors, and 2) whether individual differences in the use of these cognitive styles would explain the relationship between positive/negative self-event connections and psychological distress. The second area examined 1) whether positive and negative selfevent connections found in life story narratives would be related to meaning in life and 2) whether variations in meaning in life explained the relationship between positive/negative self-event connections and psychological well-being (as measured by satisfaction with life). Young adults were selected for this study as this developmental period is considered a critical time for the development and consolidation of autobiographical reasoning skills, and the development of psychological distress is an important concern for this population group (Arnett, 2000; 2007). 


\section{Method}

\section{Participants}

Participants were 164 undergraduate students (69\% female) enrolled in an introductory psychology course at Victoria University of Wellington, New Zealand. Participants received credit towards a course requirement of participation in research. Ages ranged from 18 to 27 years $(M=19.1$ years, $S D=2.3$ years). Seventy-two percent of participants identified as New Zealand European, 7 percent as New Zealand Maori, 3 percent as Pasifika, and 10 percent as other (one in seven participants identified with more than one ethnic background). Participants were drawn from a separate pool to those who took part in Study 1a and Study 1b. Despite this, participants were similar to those who participated in Study 1a in terms of all demographic characteristics (age, gender and ethnicity).

\section{Overview of Procedure}

The study was conducted over one 60-minute and one 30-minute session. The order in which participants completed the sessions was counterbalanced. Each session took place in a psychology laboratory; participants (with numbers ranging between two and four per session) were seated at individual desks, separated by dividers. At the start of the first session, participants read a summary of the nature and purpose of the study and provided consent. In the 60-minute session, participants completed an autobiographical narrative task through an online survey created in Qualtrics (Qualtrics Labs Inc., Provo, UT). In the 30-minute session, also online through Qualtrics, participants completed measures of attribution style, rumination, use of cognitive reappraisal strategies and a measure of psychological distress (symptoms of depression, anxiety and stress).

\section{Tasks and measures}

Explanatory style. The Attributional Style Questionnaire (ASQ; Peterson et al., 1982). This task measures the extent to which individuals assign internal versus external, stable versus unstable and global versus specific causes to positive and negative events. Participants were given six hypothetical and six hypothetical negative events and asked to record the major cause for each event. Participants are then asked to rate on a 7-point scale, the extent to which the cause of each event is internal $(1=$ "Totally due to other people or circumstances, $7=$ "Totally due to me"), stable ( 1 = "Will never again be present", 7 = "Will always be present"), and global (1= "Influences just this particular

situation, 7 = "Influences all situations in my life). Responses can then be summed for positive and negative events, with higher scores reflecting more internal, stable and global 
attributions. Higher scores for negative events reflect a negative explanatory style, and higher scores for positive events reflect a more optimistic explanatory style. The ASQ has been found to have good reliability and validity, internal consistency (Cronbach's alpha) has been shown to be reasonable for each of the subscales (.75 for positive events and .72 for negative events; Peterson et al., 1982).

Rumination. Ruminative Response Scale (RRS; Treynor, Gonzalez, \& NolenHoeksema, 2003). This scale is a 22-item self-report questionnaire which measures the extent to which individuals engage in repetitive, ruminative thinking and behaviour following feelings of distress. Participants are told that "people do a range of things when they feel sad, blue or depressed" and are asked to record on a 4-point scale ( 1 = Almost never, 4 = Almost always), the extent to which they do each of the following items. Items measure the extent to which participants act on, or think about, experiences of distress (e.g. "Go some place alone to think about your feelings") and the extent to which participants think about the cause of their distress symptoms (e.g. "Think "why do I always react this way'"). Scores are summed to provide a composite RRS score, which has been shown to have excellent internal consistency (Cronbach's alpha .92; Treynor et al., 2003).

Cognitive reappraisal. Cognitive reappraisal subscale of the Emotion Regulation Questionnaire (ERQ; Gross \& John, 2003). Five items measured the extent to which individuals habitually use reappraisal as an emotion regulation strategy. Example items include "When I want to feel more positive emotion (such as joy or amusement), I change what I'm thinking about" and "When I want to feel less negative emotion, I change the way I'm thinking about the situation". Participants rate their level of agreement on a 7point scale $(1=$ Strongly disagree, $7=$ Strongly agree $)$. Internal consistency for this subscale has been shown to be high (.82; Gross \& John, 2003).

Meaning in life. Presence of Meaning subscale of the Meaning in Life Questionnaire (MLQ; Steger, et al., 2006). Five items measured the extent to which individuals perceive that their life has a sense of purpose and meaning. Example items include :" I have a good sense of what makes my life meaningful" and "My life has no clear purpose" (reverse-coded). Participants rate how true each statement is for them on a 7-point Likert scale ( 1 = Absolutely untrue, $7=$ Absolutely true). The Presence subscale of the MLQ has been shown to have good psychometric properties (alpha reliability $=.82$; Steger et al., 2006).

Symptoms of psychological distress. Depression Anxiety Stress Scale (DASS; Lovibond \& Lovibond, 1995). This self-report questionnaire measures three related 
negative emotional states: depression, anxiety and stress, each on a 14-item subscale. Participants rate on a four-point scale how much each symptom applied to them over the past week $(0=$ Did not apply to me at all, $3=$ Applied to me very much, or most of the time). Consistent with previous research (Crawford \& Henry, 2003), a total DASS score was calculated by summing each of the subscales. Internal consistency for the DASS total score within the current sample was .98 .

Satisfaction with life. Satisfaction With Life Scale (SWLS; Diener, Emmons, Larsen, \& Griffen, 1985). This 5-item questionnaire measures individuals' subjective appraisals of the degree to which they are satisfied with their lives. Participants rate their level of agreement with each of the five statements on a 7-point Likert scale ( 1 = Strongly disagree, 7 = Strongly agree). Example items include "I am satisfied with life" and "If I could live my life over, I would change almost nothing”. The SWLS has demonstrated good psychometric properties including good internal consistency (.87; Diener et al., 1985), test-retest reliability and convergent validity (Pavot, Diener, Colvin, \& Sandvik, 1991) (Pavot \& Diener, 1993).

Autobiographical narrative task. Life story narratives were attained through an autobiographical narrative task modified from McAdams' guided autobiography and widely used in previous research (McAdams, 2006; available at http://www.sesp.northwestern.edu/foley/instruments/guided/; see also McLean \& Breen, 2009; McLean \& Lilgendahl, 2008). Participants were asked to provide written narratives of a high point, low point and turning point from their life story. High points were defined as "moments or episodes in a person's life in which he or she feels a sense of happiness, joy, excitement, contentment or some other positive emotion". Low points were defined as "moments or episodes in a person's life in which he or she feels extremely negative emotions such as despair, disillusionment, guilt, shame etc." Turning points were defined as "episodes through which a person undergoes substantial transition or change with respect to their understanding of themselves".

Participants were asked to write about each event in some detail, and to consider the following in their descriptions: how old they were when the event occurred, what happened in the event, who was involved in the event, what they were thinking/feeling/wanting in the event, why they think that this is an important event in their life story and what the event says about who they are. The order in which participants wrote about their high point, low point, and turning point was counterbalanced. 


\section{Narrative coding}

Self-event connections. Following McLean and Fournier (2008), self-event connections were coded as any statements where participants explicitly linked an aspect of the narrated event to their sense of self. Consistent with the procedure outlined in Study 1a (Chapter Four),all written narratives were coded for self-event connections by the first author. Twenty-four percent of the narratives were coded by an independent coder who was blind to the hypotheses of the study. Consistent with the approach taken in Study 1a, the number of self-event connections was calculated on a per narrative basis and inter-rater agreement for the total number of connections per narrative was kappa .82 .

Self-event connections were also coded for valence. Following the coding scheme described in Study 1a (Chapter Four), self-event connections were coded as positive when the statement referred to a positive characteristic of the self, mentioned personal growth, or denoted a positive evaluation of the self (e.g., "This event made me stronger"). Self-event connections were coded as negative when the statement referenced a negative characteristic of the self, or denoted a negative evaluation of the self (e.g., "this event is one of the main causes for the long term depression that I experience"). Self-event connections were coded as neutral when there was no evaluation of the self, or the connection was not clearly positive or negative (e.g., "this experience changed how I think about the world"). Self-event connections were coded as mixed when the connection involved elements of both positive and negative meaning. These were often situations where participants had learnt something (and thus could be considered an experience involving growth), but where the lesson learnt had negative connotations (e.g. "This experience taught me about the harsh realities of life").

Self-event connections were only recorded as positive or negative if the selfcharacteristic highlighted was explicitly referred to as positive or negative by the participant (e.g., "It was a great experience because it taught me how to be independent" positive; "It has helped me over the last year, but in a negative way" - negative), or if it satisfied common cultural understandings of desirable and undesirable characteristics (e.g., "I gained a lot of self-confidence" - positive, and "This [event] has made me more paranoid" - negative). Self-event connections that were identified by both coders (89\% of connections identified by the first coder) were compared for valence. Inter-rater agreement for the valence of self-event connections was kappa .82 . 


\section{Results}

\section{Descriptive statistics}

On average, narratives of low points $(M=199$ words, $S D=118$ words, range $=33$ -623 words $)$ and narratives of turning points $(M=202$ words, $S D=116$ words, range $=$ $28-654$ words) were significantly longer than narratives of high points ( $M=165$ words, $S D=84$ words, range $=34-443$ words; $t(163)=4.86, p<.05$ and $t(162)=4.646, p<$ .05 respectively). Consistent with the approach taken in prior research, the total number of self-event connections for each event type, and the mean, standard deviation and range of the number of self-event connections per narrative type is presented in Table 6.1 (McLean et al., 2010; Berntsen \& Rubin, 2006; Boals, 2010). On average, more self-event connections were found in narratives of low points $(M=1.50, S D=1.00 ; t(163)=3.32, p$ $<.05)$ and narratives of turning points $(M=1.60, S D=.90 ; t(162)=4.57, p<.05)$ than in narratives of high points ( $M=1.20, S D=.95$ words). The average number of self-event connections found in each event type were slightly lower than the number identified in prior work (McLean \& Fournier, 2008), yet this is consistent with the number of self-event connections reported in Study $1 \mathrm{a}^{9}$.

Of note, participants made more positive self-event connections overall than they did negative self-event connections, $(t(163)=16.25, p<.05)$. Where they were made, negative self-event connections were more likely to be found in narratives of low points than they were in narratives of turning points, $(t(163)=2.31, p<.05)$, and narratives of high points, $(t(163)=4.83, p<.05)$. Positive self-event connections were more likely to be made in narratives of turning points than in narratives of low points, $(t(163)=3.02, p<$ $.05)$, but equally likely to be made in narratives of high points.

One point of comparison of note between the participants in the current study and those who participated in Study 1a is the levels of reported psychological distress. On average, participants in the current study reported significantly lower levels of psychological distress $(M=23.65, S D=19.90)$ than participants from Study 1a $(M=$ $32.60, S D=29.65 ; t(118)=2.74, p<.05)$.

\footnotetext{
9 See Chapter Four, page 63 for a possible explanation
} 
Table 6.1

Means, standard deviations and ranges for self-event connections across memory type Self-event connections

\begin{tabular}{lrrrrrr} 
& \multicolumn{5}{c}{ Positive } & \multicolumn{5}{c}{ Negative } \\
\cline { 2 - 5 } & Sum & M (SD) & Range & Sum & M (SD) & Range \\
High points & 184 & $1.12(.94)$ & $0-4$ & 6 & $.04(.22)$ & $0-2$ \\
Low points & 160 & $.98(.97)$ & $0-4$ & 48 & $.29(.63)$ & $0-3$ \\
Turning points & 203 & $1.24(.87)$ & $0-4$ & 27 & $.16(.50)$ & $0-3$ \\
\hline Total & 547 & $3.34(1.96)$ & $0-10$ & 81 & $.49(.90)$ & $0-5$ \\
\hline & & & Self-event connections & \\
\hline & Sum & M (SD) & Range & Sum & M (SD) & Range \\
\hline High points & 3 & $.02(.17)$ & $0-2$ & 3 & $.02(.13)$ & $0-1$ \\
Low points & 13 & $.08(.27)$ & $0-1$ & 22 & $.13(.41)$ & $0-2$ \\
Turning points & 14 & $.09(.28)$ & $0-1$ & 19 & $.12(.34)$ & $0-2$ \\
\hline Total & 30 & $.18(.45)$ & $0-3$ & 44 & $.27(.52)$ & $0-2$ \\
\hline
\end{tabular}




\section{Correlations between autobiographical reasoning valence, cognitive response styles and meaning in life}

Pearson correlations examined the hypotheses that the number of positive and negative self-event connections found in young adults' life story narratives would be associated with the three cognitive response factors and meaning in life ratings (Table 6.2). As predicted, the number of positive self-event connections found in narratives of low points was associated with adopting a positive explanatory style and greater use of cognitive reappraisal strategies. Also as predicted, the number of negative self-event connections found in narratives of low points was positively associated with rumination and inversely associated with adopting a positive explanatory style and less use of cognitive reappraisal strategies. Finally, as predicted, the number of negative self-event connections found in narratives of turning points were also positively associated with rumination and inversely associated with use of cognitive reappraisal strategies. Contrary to predictions, neither the number of positive nor the number of negative self-event connections found in narratives of high, low and turning points were associated with meaning in life ratings. The total number of negative self-event connections made across all event types however (high, low and turning points combined) was associated with less meaning in life.

Pearson correlations then examined key associations between positive/negative selfevent connections, the cognitive variables, meaning in life and the two indicators of psychological functioning; DASS scores and Satisfaction with life ratings (Table 6.3). These correlations were performed to a) establish expected relationships between cognitive response styles and psychological distress, relationships between meaning in life and psychological well-being and b) to replicate the relationships between autobiographical reasoning valence and psychological functioning found in Study 1a. As predicted, symptoms of psychological distress were positively associated with a negative explanatory style and rumination, and inversely associated with use of cognitive reappraisal strategies and meaning in life. Also as predicted, satisfaction with life ratings were positively associated with meaning in life. Satisfaction with life ratings were also positively associated with positive explanatory style and use cognitive reappraisal strategies, and inversely associated with a negative explanatory style and rumination. 
Table 6.2

Correlations amongst narrative and cognitive variables

\begin{tabular}{|c|c|c|c|c|c|c|c|c|c|c|c|c|c|}
\hline & 1 & 2 & 3 & 4 & 5 & 6 & 7 & 8 & 9 & 10 & 11 & 12 & 13 \\
\hline 1. Negative explanatory style & - & & & & & & & & & & & & \\
\hline 2. Positive explanatory style & -.03 & - & & & & & & & & & & & \\
\hline 3. Rumination & $.25 * *$ & -.06 & - & & & & & & & & & & \\
\hline 4. Cognitive reappraisal & $-.16^{*}$ & $.28 * *$ & -.11 & - & & & & & & & & & \\
\hline 5. Meaning in life & $-.23 * *$ & $.22 * *$ & $-.27 * *$ & $.31 * *$ & - & & & & & & & & \\
\hline \multicolumn{14}{|l|}{ Positive connections: } \\
\hline 6. Turning points & $-.24 * *$ & .08 & -.06 & .14 & .14 & - & & & & & & & \\
\hline 7. Low points & -.08 & $.21 * *$ & -.08 & $.19 *$ & .08 & $.27 * *$ & - & & & & & & \\
\hline 8. High points & -.10 & .09 & .10 & .11 & .03 & .14 & $.33 * *$ & - & & & & & \\
\hline 9. Total positive & -.19 & $.18 *$ & -.02 & $.21 * *$ & .11 & $.65 * *$ & $.77 * *$ & $.70 * *$ & - & & & & \\
\hline \multicolumn{14}{|l|}{ Negative connections: } \\
\hline 10. Turning points & .12 & -.10 & $.19 *$ & $-.18 *$ & -.17 & $-.23 * *$ & .02 & .06 & -.06 & - & & & \\
\hline 11. Low points & .11 & $-.26 * *$ & $.18 *$ & $-.21 * *$ & -.15 & -.13 & $-.23 * *$ & .12 & -.12 & $22 * *$ & - & & \\
\hline 12. High points & -.05 & -.04 & .01 & -.03 & -.13 & -.01 & .12 & .01 & .06 & .00 & -.08 & & \\
\hline 13. Total negative & .13 & $-.25 * *$ & $.23 * *$ & $-.25 * *$ & $-.23 * *$ & $-.22 * *$ & -.12 & .12 & -.10 & $.71 * *$ & $.80 * *$ & $.19 *$ & \\
\hline
\end{tabular}

$* p<.05, * * p<.01$ 
Replicating the findings of Study 1a, symptoms of psychological distress were positively associated with the number of negative self-event connections found in narratives of turning points and low points (and the number of negative connections found overall). Similar associations were found in relation to satisfaction with life. Satisfaction with life ratings were inversely associated with the number of negative self-event connections found in narratives of low and turning points. Unlike symptoms of psychological distress, satisfaction with life ratings were also positively associated with the number of positive self-event connections found in narratives of low points.

Table 6.3

Correlations amongst narrative variables and psychopathology/well-being scales

Psychological distress Psychological well-being

(DASS scores) (Satisfaction with life ratings)

\begin{tabular}{lll}
\hline 1. Negative explanatory style & $.29^{* *}$ & $-.22^{* *}$ \\
2. Positive explanatory style & -.10 & $.31^{* *}$ \\
3. Rumination & $.62^{* *}$ & $-.38^{* *}$ \\
4. Cognitive reappraisal & $-.16^{*}$ & $.28^{* *}$ \\
5. Meaning in life & $-.29^{* *}$ & $.54^{* *}$ \\
Positive connections: & & \\
6. Turning points & -.03 & .15 \\
7. Low points & -.14 & $.16^{*}$ \\
8. High points & .01 & .03 \\
9. Total positive & -.08 & $.16^{*}$ \\
Negative connections: & & $.23^{* *}$ \\
10. Turning points & $.22^{* *}$ & $-.24^{* *}$ \\
11. Low points & $.16^{*}$ & -.02 \\
12. High points & -.02 & \\
13. Total negative & & \\
\hline
\end{tabular}

$* p<.05, * * p<.01$ 


\section{Significant predictors of psychological distress}

Multiple regression analyses were employed to examine the hypothesis that positive/negative self-event connections made in narratives of high, low and turning points would not explain unique variance in psychological distress (DASS scores) over and above that which is explained by the cognitive response factors. The three cognitive variables (negative explanatory style, rumination, cognitive reappraisal) and the self-event connections with a significant association to DASS scores (negative connections in turning points and low points) were entered simultaneously as predictor variables. Only those self-event connections that were significantly associated with DASS scores in Table 6.3 were included in the analysis to minimise the risk of over-fitting the regression equation. Total DASS scores were entered as the dependent variable.

Two predictors (negative explanatory style and rumination) explained $40 \%$ of the variance in DASS scores $\left(R^{2}=.40, F(2,161)=53.95, p<.05\right)$. In this sample of young adults, symptoms of psychological distress were predicted by greater endorsement of a negative explanatory style $(\beta=.14, p<.05)$ and higher levels of rumination about the causes of emotional distress $(\beta=.58, p<.05)$. The number of negative self-event connections found in narratives of turning points and low points did not account for unique variance in DASS scores.

\section{Significant predictors of psychological well-being (satisfaction with life)}

Multiple regression also examined the hypothesis that the number of positive and negative self-event connections made in narratives of high, low and turning points would not explain unique variance in psychological well-being (satisfaction with life ratings) over and above that which is explained by participants appraisal of meaning in their lives. Again, as this analysis was exploratory in nature, meaning in life scores and the self-event connections with a significant association to psychological well-being (negative connections in turning points and low points and positive connections in low points) were entered simultaneously as predictor variables. Only those self-event connections that were significantly associated with well-being scores in Table 6.3 were included in the analysis to minimise the risk of overfitting the regression equation. Satisfaction with life was entered as the dependent variable.

Two predictors (meaning in life and negative self-event connections found in narratives of low points) explained $43 \%$ of the variance in Satisfaction with life ratings $\left(R^{2}=\right.$ $.33, F(2,160)=38.99, p<.05)$. In this sample of young adults, satisfaction with one's life were predicted by higher levels of meaning in life $(\beta=.51, p<.05)$ and lower numbers of negative self-event connections in low point narratives $(\beta=-.20, p<.05)$. The number of 
positive self-event connections found in narratives of low points did not account for unique variance in satisfaction with life scores.

The relationship between negative self-event connections and psychological distress mediating and/or moderating role of negative explanatory style?

Moderation analyses tested whether negative explanatory style moderated the relationship between negative self-event connections made in narratives of low and turning points and symptoms of psychological distress (DASS scores). The first step in exploring the moderation effect was to conduct a regression analysis, with the number of negative selfevent connections found in narratives of low and turning points in the first step (main effect), negative explanatory style entered in the second step (moderating variable). The two-way interaction (negative self-event connections $x$ negative explanatory style) was entered in the third step and total DASS scores were entered as the dependent variable. Consistent with the recommendations of Aiken and West (1991), each variable was centred before being entered into the regression model.

Significant main effects were found for negative self-event connections $(\beta=.16, p<$ $.05)$ and negative explanatory style $(\beta=.25, p<.05)$, confirming the basic relationships that making negative connections in narratives of low turning points and adopting a negative explanatory style were both individually associated with more symptoms of psychological distress. The interaction between negative self-event connections and negative explanatory style was significant at the $10 \%$ level $(\beta=.15, p=.06)$. Since moderation effects were predicted, a decision was made to graph the findings and conduct simple slope analyses. Simple slope analysis (Figure 6.1) revealed that adopting a negative explanatory style exacerbated the relationship between the number of negative self-event connections in narratives of low and turning points and DASS scores. The slopes for the high and moderate use of a negative explanatory style were significantly different from zero (slopes $=2.16$ to 3.33 , ps <.05) showing that for young adults who were the most likely (and moderately likely) to adopt a negative explanatory style, making more negative self-event connections in narratives of low and turning points was associated with higher levels of psychological distress symptoms. The slope for the low use of a negative explanatory style was not significantly different from zero (slope $=0.23, p>.05$ ) showing that for young adults who did not characteristically adopt a negative explanatory style, making more negative self-event connections in narratives of low and turning points was not associated with an increase in symptoms of psychological distress. 


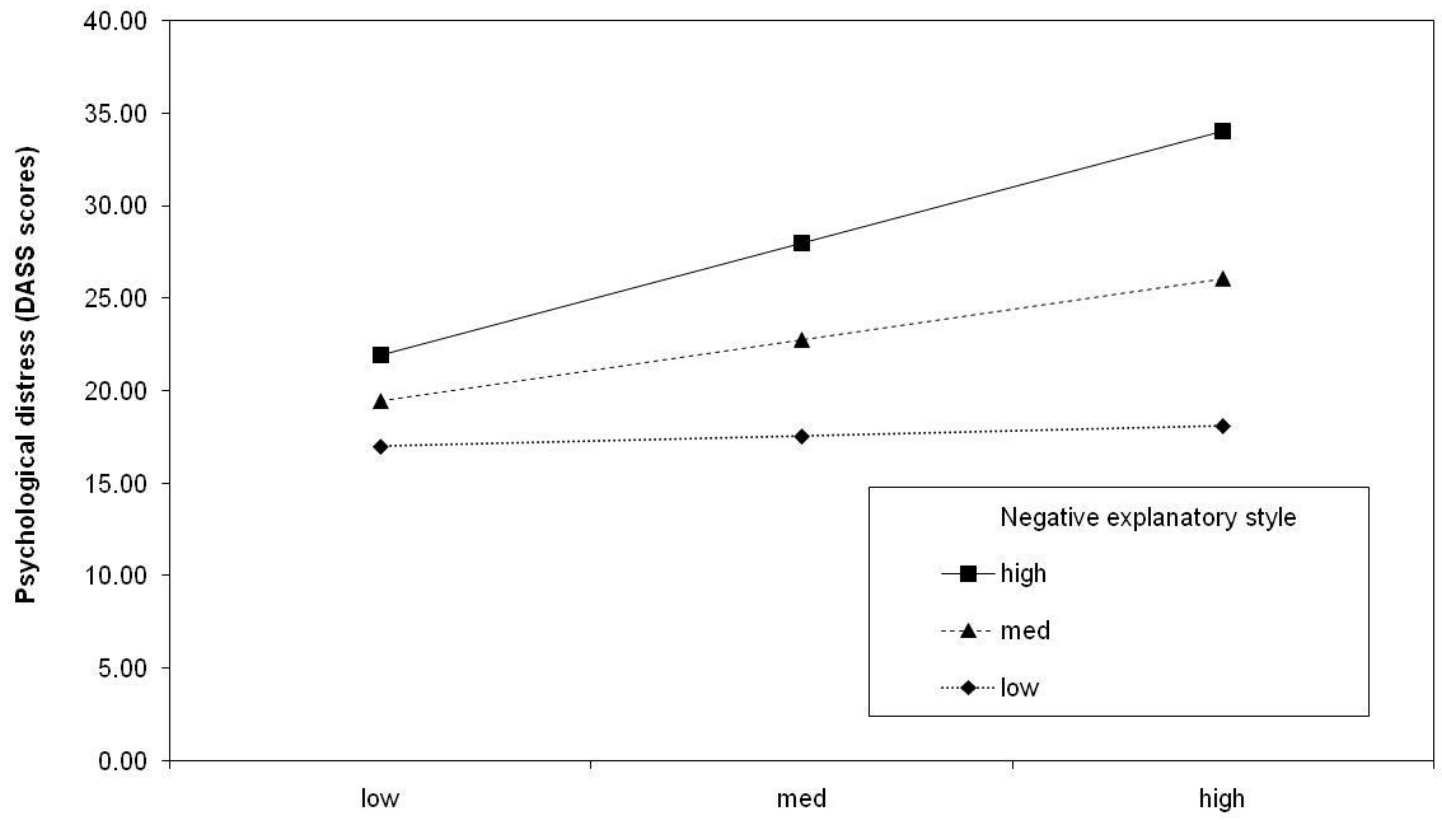

Number of negative self-event connections in narratives of low and turning points

Figure 6.1. Moderation of relationship between number of negative self-event connections found in narratives of low and turning points and psychological distress, by negative explanatory style

Mediation analysis then explored whether negative explanatory style acted as a mechanism through which the number of negative self-event connections found in narratives of low and turning points predicted psychological distress. The first step in the mediation analysis was to conduct correlations between the three variables (negative explanatory style, number of negative self-event connections in low and turning points combined, and psychological distress). All three variables were significantly correlated at the $10 \%$ level ( $r s$ $=.14$ to .29 , ps <.07). When entered into a mediation model however, no significant mediation effect was detected (Sobel's $z=1.59, p>.05$ ). The indirect path from negative self-event connections through negative explanatory style to DASS scores was nonsignificant (.04). The direct path between negative self-event connections and DASS scores remained significant when negative explanatory style was included in the analysis $(r=.20, p$ $<.05)$. 


\section{The relationship between negative self-event connections and psychological distress - mediating and/or moderating role of rumination?}

Regression analyses tested whether the relationship between the number of negative self-event connections found in narratives of low and turning points and psychological distress was moderated by rumination. The number of negative self-event connections found in narratives of low and turning points was entered in the first step, rumination was entered in the second step, and the two-way interaction (negative self-event connections $\mathrm{x}$ rumination) was entered in the third step. Total DASS scores were entered as the dependent variable. A significant main effect was found for rumination $(\beta=.59, p<.05)$, confirming the basic relationship that symptoms of psychological distress are significantly higher among young adults who tend to ruminate more. There was no main effect for the number of negative selfevent connections and nor was there a significant interaction between the number of negative connections and rumination $(\beta \mathrm{s}=.08, p \mathrm{~s}>.05)$. As no significant interaction was detected, graphing of moderation effects were not pursued.

Mediation analysis then explored whether rumination acts as a mechanism through which the number of negative self-event connections made in narratives of low and turning points predicts symptoms of psychological distress. All three variables (number of negative self-event connections in low and turning points combined, rumination and psychological distress) were significantly correlated ( $r s=.24$ to $.62, p s<.07$ ). As shown in Figure 6.2, rumination significantly mediated the relationship between the number of negative self-event connections and symptoms of psychological distress (Sobel's $z=2.94, p<.05$ ). The indirect path from the number of negative self-event connections, through rumination, to symptoms of psychological distress was found to be significant (.14). This path shows that making more negative self-event connections in narratives of low and turning points predicted higher levels of rumination, which in turn predicted higher levels of psychological distress. The direct path from number of negative self-event connections to psychological distress was reduced to nonsignificance when rumination was accounted for within the model $(r=.10, p>.05)$. This result suggests that the number of negative self-event connections made in low and turning point narratives was unrelated to DASS scores when rumination was included in the model. 


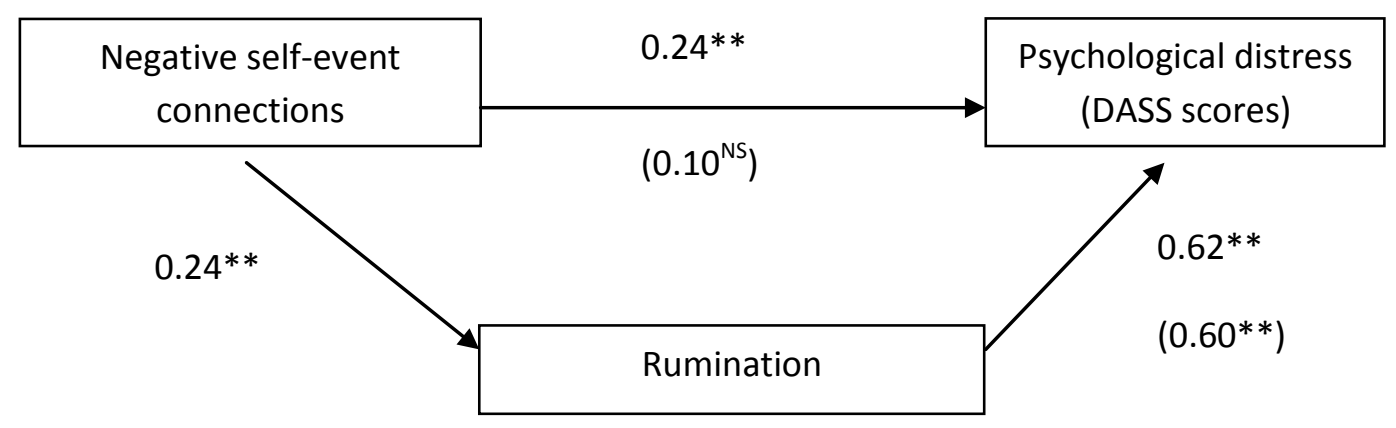

Note: The numbers presented in the model are beta weights. $* * \mathrm{p}<.05$

Figure 6.2. Mediation of the relationship between the number of negative self-event connections in narratives of low and turning points and psychological distress, by rumination

The relationship between positive/negative self-event connections and psychological distress - mediating and/or moderating role of cognitive reappraisal strategies?

Regression analyses then tested whether the relationship between the number of positive self-event connections found in narratives of low and turning points and symptoms of psychological distress were moderated by use of cognitive reappraisal strategies. The number of positive self-event connections found in narratives of low and turning points was entered in the first step, use of cognitive reappraisal strategies was entered in the second step, and the two-way interaction (negative self-event connections $\mathrm{x}$ cognitive reappraisal) was entered in the third step. Total DASS scores were entered as the dependent variable. No significant main effects were found for number of positive connections in narratives of low and turning points or for cognitive reappraisal strategies $(\beta s=-.12$ to $-.10, p s>.05)$, nor was a significant interaction (positive connections $\mathrm{x}$ cognitive reappraisal) found $(\beta=.07, p>$ .05). As no significant main effects or an interaction were detected, graphing of the moderation effect was not pursued.

Mediation analysis then explored whether cognitive reappraisal strategies acted as a mechanism through which the number of positive self-event connections made in narratives of low and turning points predicted lower levels of psychological distress. A precondition for testing mediation effects is that all variables involved in the mediation should be significantly correlated. This precondition precluded the mediating effect of cognitive reappraisal strategies on the positive connections to DASS relationship being tested as the number of 
positive self-event connections made in narratives of low and turning points was not significantly associated with DASS scores $(r=-.11, p>.05)$.

\section{The relationship between positive self-event connections and psychological well-being - mediating and/or moderating role of meaning in life?}

Regression analyses then tested whether the relationship between the number of positive self-event connections found in narratives of high, low and turning points (combined) and psychological well-being (as measured by Satisfaction with life) was moderated by meaning in life. The total number of positive self-event connections found in narratives of high, low and turning points was entered in the first step, meaning in life was entered in the second step, and the two-way interaction (positive self-event connections $\mathrm{x}$ meaning in life) was entered in the third step. Total Satisfaction with Life ratings were entered as the dependent variable.

Significant main effects were found for positive self-event connections $(\beta=.14, p<$ $.05)$ and meaning in life $(\beta=.51, p<.05)$, confirming the basic relationships that making positive connections in narratives of high, low turning points and the perception that one's life has meaning are both individually associated with increased levels of satisfaction with life. The interaction between positive self-event connections and meaning in life was significant at the $10 \%$ level $(\beta=-.12, p=.09)$, so moderation effects were pursued. Simple slope analysis (Figure 6.3) revealed that for young adults who report an absence or small amount of meaning in life, making more positive self-event connections in narratives of high, low and turning points from the life story was associated with higher life satisfaction (slopes $=2.06$ to 2.15 , ps <.05). The slope for the high level of meaning in life was not significantly different from zero $($ slope $=0.11, p>.05$ ) showing that for young adults who reported a high level of meaning in life, making more positive self-event connections in narratives of high, low and turning points was not associated with higher satisfaction with life.

This moderation analysis was then repeated for the total number of negative self-event connections found in narratives of high, low and turning points (combined). The total number of negative self-event connections found in narratives of high, low and turning points was entered in the first step, meaning in life was entered in the second step, and the two-way interaction (positive self-event connections x meaning in life) was entered in the third step. Total DASS scores were entered as the dependent variable. Significant main effects were found for negative self-event connections $(\beta=-.22, p<.05)$ and meaning in life $(\beta=.49, p$ $<.05$ ), confirming the basic relationships that making negative connections in narratives of high, low turning points was associated with lower levels of satisfaction with life and the 
perception that one's life has meaning was associated with increased levels of satisfaction with life. The interaction between negative self-event connections and meaning in life was not significant $(\beta=-.01, p>.05)$, so moderation effects were not further pursued.

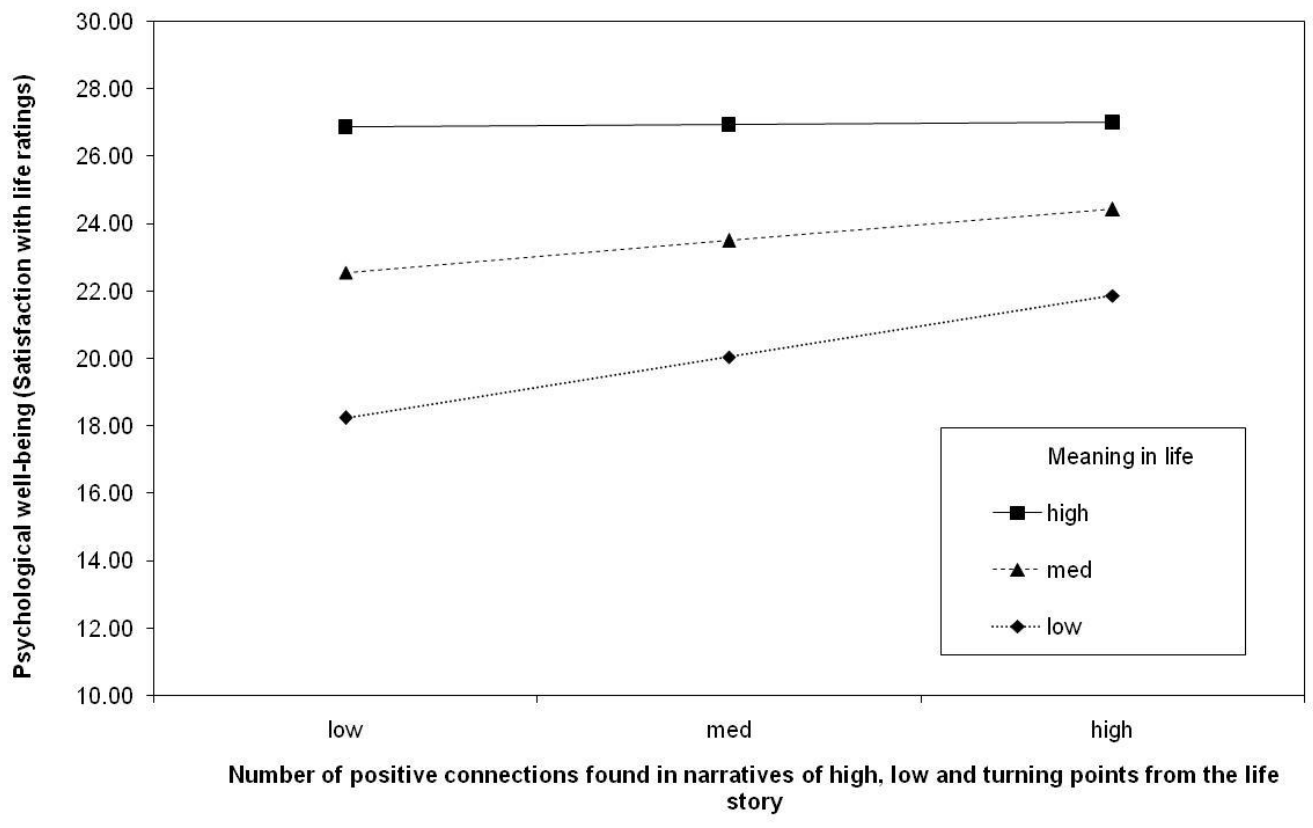

Figure 6.3. Moderation of relationship between number of positive self-event connections found in narratives of high, low and turning points and satisfaction with life, by meaning in life

Mediation analysis then explored whether meaning in life acted as a mechanism through which the number of positive self-event connections made in narratives of high, low and turning points (combined) predicted satisfaction with life. The precondition for testing mediation effects in relation to positive connections was not met as the total number of positive self-event connections made in narratives of high, low and turning points was not significantly associated with meaning in life ratings $(r=.11, p>.05)$.

The precondition for testing mediation effects in relation to negative connections was met however. All three variables (number of negative self-event connections in high, low and turning points combined, meaning in life ratings and satisfaction with life) were significantly correlated ( $r s=-.33$ to $.54, p s<.05$ ). As shown in Figure 6.4 , meaning in life partially mediated the relationship between the number of positive self-event connections and satisfaction with life ratings (Sobel's $z=-2.75, p<.05$ ). The indirect path from the number of negative self-event connections, through meaning in life, to satisfaction in life was 
significant (-.11). This path shows that young adults who made fewer negative self-event connections in narratives of high, low and turning points reported more meaning in life, which in turn was associated with increased satisfaction with life. The direct path from number of negative self-event connections to satisfaction in life was reduced, but not to nonsignificance when meaning in life was included in the model $(r=-.22, p>.05)$. This suggests that meaning in life ratings partially accounted for the relationship between fewer negative self-event connections made in high, low and turning point narratives and satisfaction with life.

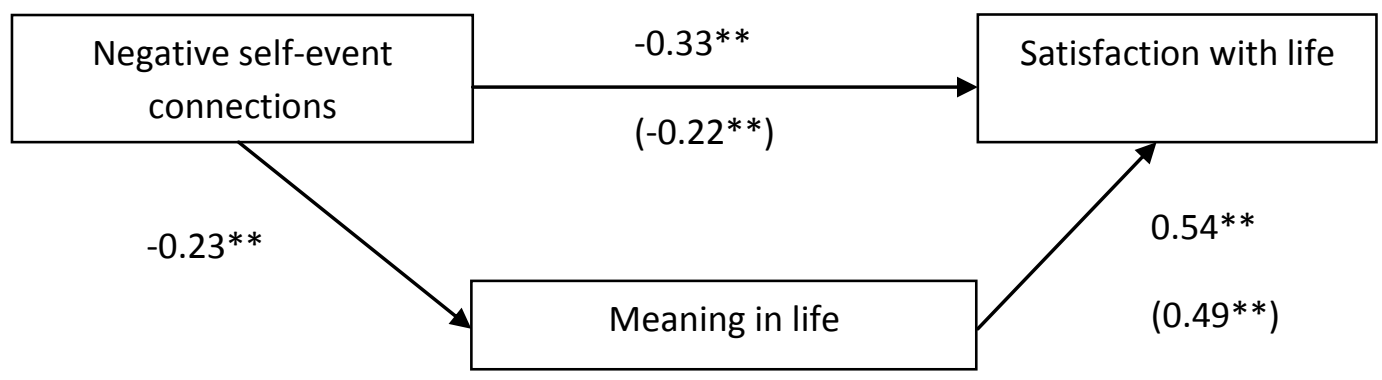

Note: The numbers presented in the model are beta weights. $* * \mathrm{p}<.05$

Figure 6.4. Mediation of the relationship between negative self-event connections in narratives of high, low and turning points and Satisfaction with Life ratings by meaning in life ratings

\section{Discussion}

There were two main areas of investigation in the current study. The first area examined whether autobiographical reasoning valence (as measured by positive and negative self-event connections) was related to each of the three cognitive response factors and meaning in life. The second area examined whether cognitive response factors and meaning in life ratings mediated and/or moderated the relationship between the valence of autobiographical reasoning found in young adults' life story narratives and psychological functioning. By examining these associations, I aimed to investigate whether our understanding of the link between psychological functioning and autobiographical reasoning valence could be enhanced by considering additional factors that may be involved in this relationship. Before considering findings in relation to these aims, the question of whether the basic relationship between autobiographical reasoning valence and psychological functioning outline in Study 1a was replicated will be addressed. 


\section{Relationship between autobiographical reasoning valence and psychological functioning}

In the current study, participants were asked to narrate a third event type, turning points, in addition to low and high points. Turning points may demonstrate a stronger relationship to autobiographical reasoning than low or high points because they involve a substantial change to the individual or their life and such changes, in a theoretical sense, are likely to be explained by autobiographical reasoning (McAdams et al., 2001; McLean \& Pratt, 2006).

As predicted, the overall number of negative self-event connections found in young adults' life story narratives (high points, low points and turning points combined) was positively associated with symptoms of psychological distress. When analysed by event type, symptoms of psychological distress were only associated with the number of negative selfevent connections found in narratives of low points and turning points. This is consistent with prior work highlighting that the manner in which negative events are processed has greater implications for psychological functioning than the processing of positive events (e.g. Lyubomirsky, Sousa \& Dickerhoof, 2006; Pals, 2006).

Unexpectedly, the number of positive self-event connections made in narratives of high, low, and turning points (both separately and combined), was unrelated to symptoms of psychological distress. These findings contradict those presented in Study 1a, which showed that symptoms of psychological distress were lower among young adults who made more positive self-event connections. This inconsistency in findings may reflect differences in the level of distress reported by each of the two samples. Participants in Study 1a reported higher scores on the Depression Anxiety Stress Scale (DASS; Lovibond \& Lovibond, 1995) and a higher range of scores than participants in the current study (average score of 33 in Chapter Three, and 23 in the current study). The limited range of DASS scores present in the current study may have limited the extent to which relationships between positive connections and distress symptoms could be observed.

\section{Relationships between autobiographical reasoning valence, cognitive response styles and psychological functioning \\ Negative explanatory style.}

Consistent with prior work, adopting a negative explanatory style was positively associated with symptoms of psychological distress (depression, anxiety and stress combined) (Luten at al., 1997; Mongrain \& Blackburn, 2005; Palker-Corell \& Marcus, 2004; Runyon \& Kenny, 2002). Unexpectedly, adopting a negative explanatory style was unrelated to the number of negative self-event connections found in narratives of low and turning 
points from the life story. Relatedly, negative explanatory style did not mediate the relationship between the number of negative self-event connections found in narratives of low and turning points and psychological distress. Although direct comparisons to prior work cannot be made due to unique style of coding self-event connection valence used in this study, the non-significant relationship between negative self-event connections and negative explanatory style is consistent with the non-significant relationship between negative explanatory style and contamination sequences found by Adler et al. (2006). These findings converge on the notion that autobiographical reasoning and explanatory style represent different cognitive processes.

Pasupathi, Mansour and Brubaker (2007) made the distinction between self-event connections that describe a change in the self as a result of an experience and self-event connections that explain why an event happened (i.e. a stability connection). These two types of self-event connections are highlighted in the following examples:

"My grandmother died when I was around 13 years old ... I was very close
to her even though she lived four hours away ... this event kinda made me
numb to pain ... I think this has a negative impact on my life though because
I find it hard to care about other people even when in relationships with
them" (change connection)

"I was 19 when I drunkenly smashed a window walking home by myself and had to spend a night in hospital so that my hand could be sewn up ... I felt embarrassed and ashamed that I'd lost control and that I couldn't take care of myself, or couldn't responsibly manage my own drinking. It probably happened because I can be immature in approaching certain situations and not think through some decisions properly" (stability connection)

In considering the difference between these two types of self-event connections, it is clear that adopting a negative explanatory style is similar to making stability connections. In the above example, the young adult attributes the event (smashing a window while drunk) to internal, stable and global characteristics of the self (immaturity and impulsivity - not thinking through one's decisions). In the example of a change connection, however, the young adult does not make an attribution regarding the cause of the event, but instead makes 
a causal link between the event and the development of a self-characteristic (emotional disengagement from others) which they appraise as being negative. The non-significant relationship between negative explanatory style and negative self-event connections may therefore reflect the notion that adopting a negative explanatory style is expressed as stability connections, but is unrelated to explain connections. Informal observations of the current data suggest that self-event connections describing self-change were more common than selfevent connections that describe stable characteristics of the self. The small number of negative self-event connections that described stability in the self and attribute the event to these stable features of the self would limit the likelihood of such an association being detected.

Adopting a negative explanatory style did, however, moderate the relationship between the number of negative self-event connections found in narratives of low and turning points and symptoms of psychological distress. For young adults who showed the strongest tendency to attribute the cause of negative events to internal, stable and global aspects of the self, making more negative self-event connections in narratives of low and turning points was associated with an increase in symptoms of psychological distress. For young adults who were less likely to attribute the cause of negative events to internal, stable and global aspects of the self, making more negative self-event connections was not related to an increase or decrease in symptoms of distress.

The significant moderation effect suggests that although adopting a negative explanatory style and making negative self-event connections are not related to each other, psychological distress is significantly predicted by the interaction of these factors. Individuals who adopt a negative explanatory style have been shown to demonstrate an attentional bias towards negative self-relevant information. In one study, individuals who scored highly on a measure of negative explanatory style attended better to negative adjectives (e.g. failure) than neutral words when attentional resources were low (Romens, MacCoon, Abramson, \& Pollak, 2011). This heightened attention to negative information about the self may thus present as an additional risk factor for psychological distress, as negative self-related information becomes more salient than neutrally valenced information. Following this, making negative self-event connections may be attended to more strongly for young adults who tend to adopt a negative explanatory style, while other aspects of the event receive comparably less attention. This increased heightened focus on, and subsequent salience of, negative information about the self may account for the increase in psychological distress for these individuals. 


\section{Positive explanatory style.}

Adopting a positive explanatory style was not related to symptoms of psychological distress. The absence of a significant relationship is not surprising given that the positive explanatory style is not as conceptually sound, and has not demonstrated as strong a relationship to psychological distress, as its negative counterpart (Johnson, et al., 1998; Needles \& Abramson, 1990). Young adults who were more likely to adopt a positive explanatory style did however, make more positive self-event connections in narratives of low points relative to young adults who tended not to use a positive explanatory style. Limited research investigating positive explanatory style has shown an association between this cognitive factor and positive appraisals regarding the outcome of negative or traumatic events. Specifically, Ho, Chan, Yau and Yeung (2011) found that adopting a positive explanatory style is associated with post-traumatic growth, defined as beliefs about positive outcomes for the self e.g. personal strength as a result of negative experiences. In explaining these findings, Ho et al, (2011) suggest that a tendency to adopt a positive explanatory style aids the development of a "habitual reflex" which enables individuals to cope with difficult experiences in a positive way (i.e. perceive positive changes from negative experiences). Following this interpretation, making positive self-event connections in narratives of difficult life experiences (low and turning points) may be associated with a habitual tendency to think about the self in generally positive terms.

The association between positive explanatory style and positive self-event connections in low points, but not high points, is somewhat surprising given that a positive explanatory style is defined in terms of attributing the causes of positive events to internal, global and stable characteristics of the self. As highlighted by the findings of the current study and those presented in Study 1a (Chapter Four) however, self-event connections are much less likely to be made in narratives of high points than they are in narratives of low points. The low number of self-event connections made in narratives of high points likely limited the extent to which such an association could be detected.

\section{Rumination.}

Consistent with a large body of previous research (Marks, Sobanski, \& Hine, 2010; McLaughlin, Borkovec, \& Sibrava, 2007; Watkins, 2009), rumination was strongly and significantly associated with symptoms of psychological distress. Young adults who were more likely to ruminate in response to feelings of distress made more negative self-event connections in turning and low point narratives than young adults who do not tend to ruminate. Rumination also significantly (and fully) mediated the relationship between the 
number of negative self-event connections made in narratives of low and turning points and symptoms of psychological distress. This full mediation effect suggests that young adults who make more negative self-event connections in narratives of low and turning points tend to ruminate more about their feelings of distress, which in turn is associated with higher levels of psychological distress. Highlighting the full mediation effect, the direct relationship between negative self-event connections and symptoms of psychological distress was reduced to non-significance when rumination was entered into the model. This finding suggests that negative self-event connections are not uniquely associated with distress symptoms outside of the relationship which includes rumination.

One possible interpretation of these findings is that rumination is an underlying mechanism which explains why young adults who make negative self-event connections experience symptoms of psychological distress. That is, young adults who make more negative self-event connections in narratives of low and turning points may experience more psychological distress because they ruminate about the cause of their distress. The content of negative self-event connections may become entwined in the process of rumination as individuals may attribute the cause of their distress, at least in part, to the negative characteristics of the self described in negative self-event connections. This interpretation is consistent with prior work showing that for individuals experiencing psychological distress, ruminative self-focus is associated with an increase in negative judgements about the self (Rimes \& Watkins, 2005).

It is important to note that the mediation model tested in the current study is just one way of presenting the directionality of the relationship between negative self-event connections, rumination and psychological distress. The cross-sectional nature of the data precludes firm conclusions being made about the direction of causality between these three variables. From a theoretical perspective, another mediation model in which rumination leads to an increased number of negative self-event connections being made in life story narratives, which in turn may relate to an increase in distress symptoms, is possible. Longitudinal data is needed to compare the model tested in the current study with alternative models that conceptualise negative self-event connections as the mediating variable.

Rumination did not moderate the relationship between the number of negative selfevent connections in narratives of low and turning points and psychological distress. The absence of a moderation effect suggests that the relationship between the number of negative self-event connections found in narratives of low and turning points did not depend, or was not altered by, different levels of rumination. This finding is surprising in light of cognitive 
models of psychopathology which suggest that depressed affect is intensified by the interaction between negative cognitions about the self and rumination (Ciesla\& Roberts, 2007). Ciesla and Roberts (2007) found a positive association between dysphoric affect and dysfunctional (negative) attitudes when rumination was also present, while no such association was found when rumination was absent.

There are, however, some obvious differences between the current study and that of Ciesla and Roberts (2007). In the latter study, dysfunctional attitudes were assessed as an indicator of negative schematic content. The measure of dysfunctional attitudes used by Ciesla and Roberts (2007) assesses rigid beliefs commonly held by distressed individuals (e.g. "People will think less of me if I make a mistake") but do not necessarily assess negative thoughts about one's qualities or characteristics. Thus negative self-event connections and dysfunctional beliefs as assessed by the Dysfunctional Attitudes Scale (see de Graaf, Roelofs, \& Huibers, 2009) may not be measuring the same construct. Second, Ciesla and Roberts (2007) induced rumination via an experimental manipulation whereas the current study assessed young adults' self-reported engagement in rumination. Thus the moderating effect of rumination on the relationship between negative cognition and psychological distress may be observed in 'real-time' measurement of rumination, but not in self-reported assessments of one's general tendency to ruminate which are retrospective in nature. One way of exploring this possibility in future research would be to substitute the self-report measure of rumination with a rumination induction task such as that used by Ciesla and Roberts (2007).

\section{Cognitive reappraisal strategies.}

Consistent with previous findings, young adults who characteristically engaged in cognitive reappraisal strategies experienced fewer symptoms of psychological distress than those who tend not to use these strategies. As expected, the number of positive self-event connections made in narratives of low points was positively associated with use of cognitive reappraisal strategies. This finding is consistent with previous work showing that individuals who characteristically use cognitive reappraisal strategies express more positive views of themselves and their lives in general, than do non-appraisers (Nezlek \& Kuppens, 2008). These findings are also consistent with earlier work highlighting an association between reappraisal strategies and maintenance of a positive self-concept and self-esteem (Webster \& Tiggeman, 2003).

Cognitive reappraisal strategies did not moderate nor mediate the relationship between positive self-event connections and symptoms of psychological distress. There are a 
number of reasons for the absence of such effects. First, the current study found that psychological distress was related to negative, but not positive, self-event connections. In cognitive models of psychological distress, positive thoughts and beliefs about the self are not implicated in the development and maintenance of negative emotional states in the same way that are negative thoughts and beliefs about the self. While individuals who experience negative emotional states may concurrently experience some positive thoughts about the self, the impact of these thoughts may differ to that of negative self-related thoughts. For example, Ross, Mueller, and De Le Torre (1986) found that young adults who experienced moderate levels of depression perceived negative traits as distinct to themselves but did not perceive positive traits in the same way. Therefore, one possibility is that positive self-event connections are over-shadowed by any negative self-event connections made, as these negative statements about the self take on more self-relevant meaning than positive statements.

\section{Predicting psychological distress from cognitive and narrative variables}

The findings of the current study showed that symptoms of psychological distress were predicted by negative explanatory style and rumination, but not the number of negative self-event connections made in life story narratives. These findings suggest that the relationships between the valence of self-event connections found in life story narratives and indicators of psychological distress may be better accounted for by cognitive response styles.

Due to the cross-sectional nature of the study, the findings do not allow any conclusions about causality to be made. One possibility is that cognitive response factors such as explanatory style, rumination and cognitive reappraisal strategies pre-date and play a causal role in the use of autobiographical reasoning to draw negative/positive self-event connections. From a developmental perspective, there is evidence that two of the three cognitive response factors (explanatory style and rumination) are exhibited by children as young as 8 or 9 years old (Joiner \& Wagner, 1995). Although evidence regarding use of cognitive reappraisal strategies is more limited, young adolescents 13 years of age and over have demonstrated use of reappraisal as an emotion-regulation strategy (Rood, Roelofs, Bögels, \& Arntz, 2012). From both a theoretical perspective and according to limited empirical data, autobiographical reasoning capabilities increase in sophistication during late adolescence (McLean \& Breen, 2009). Thus it seems plausible that cognitive response styles develop first and potentially predispose young adults to reason about the self in positive or negative ways during construction of the life story. It is important to note, however, that the patterns between the cognitive response factors and psychological functioning in childhood 
mirror those observed in adults. That is, children who demonstrate a negative explanatory style and engage in rumination are more likely to have developed depressive symptoms by the time they reach young adulthood. As discussed in Chapter Four, these depressive symptoms are reciprocally likely to impact on young adults' thoughts and emotions about themselves, which in turn is likely to impact the autobiographical reasoning process.

\section{Relationships between autobiographical reasoning valence, meaning in life and psychological functioning}

As expected, young adults who reported greater meaning in their lives also reported greater levels of satisfaction with their lives. This is consistent with prior work highlighting a strong association between meaning and satisfaction in life (Steger \&Kashdan, 2007; Steger, Oishi, \& Kesebir, 2011). Unexpectedly, meaning in life was unrelated to the number of positive self-event connections found in narratives of high points, low points or turning points from the life story (separately and combined). This non-significant finding raises questions about whether the construction of meaning in relation to single life events relates to a broader sense of meaning in one's life. King, Hicks, Krull and Del Gaiso (2006) noted the difference between making a global assessment of the extent to which one's life has meaning and the subjective experience of a single day (or days) as being meaningful. These authors found that individuals' judgements about the meaningfulness of a given day were more strongly related to the amount of positive affect the individual experienced on the day than it was to global assessments of meaning in their lives. While the daily assessments of meaning were not limited to a single event, these findings highlight the possibility that individuals may not refer to memories of single events or days when making global assessments of meaning in life. In a similar way, deriving meaning from a life story event may be only part of the information individuals use when assessing the overall meaning of their lives (see Morgan \& Farsides, 2009 for other factors which may be involved in assessments of global meaning in life).

Although positive self-event connections were unrelated to meaning in life ratings, moderation analysis showed that in the absence of meaning in one's life, making positive self-event connections in narratives of high, low and turning points from the life story was associated with an increase in life satisfaction. Even with this increase in life satisfaction, however, young adults who reported the lowest levels of meaning in life were less satisfied with their lives than young adults who reported higher levels of meaning in life. These findings may suggest that even those young adults who believe that their life has little meaning overall can experience greater life satisfaction by deriving positive meaning from single experiences. A high degree of caution is needed in making this interpretation due to 
the cross-sectional nature of the data. Another likely possibility is that young adults who experience slightly higher life satisfaction, despite a relative absence of meaning in their lives, are simply more able to make positive self-event connections than young adults with comparatively lower levels of life satisfaction.

As predicted, the number of negative self-event connections in narratives of high, low and turning points (combined) was associated with the sense that one's life does not have meaning. Further, highlighting the strength of the association between negative connections and life satisfaction, both negative self-event connections and meaning in life ratings explained unique variance in satisfaction with life. These findings suggest that psychological well-being reflects not only having meaning in one's life, but also the absence of negative self-event connections in life story narratives. Individual differences in meaning in life were also shown to significantly mediate the relationship between the number of negative selfevent connections in life story narratives and low life satisfaction. That is, young adults who made more negative self-event connections in life story narratives reported less meaning in life which in turn was associated with lower levels of satisfaction with life. Again due to the correlational nature of our analysis, no firm conclusions can be made about the mechanisms underlying this mediation effect. One possibility is that making negative self-event connections reduces young adults' sense of meaning in life which in turn reduces life satisfaction. Alternatively, young adults who perceive their lives to be low on meaning may be more likely to make negative self-event connections when narrating life story events.

Due to the relatively small sample size, each of the moderation and mediation effects was examined separately. While this was acceptable given the exploratory nature of the study, the use of structural equation modelling to examine these effects in combination would provide a clearer picture of how all of these variables interact. In past research and in the current study, many of the cognitive, narrative and meaning variables were significantly associated with each other, and with both indicators of psychological functioning (Chang \& Sanna, 2007; Gross and John, 2003). Incorporating a number of these variables into a path model using structural equation modelling would enable key (potentially) causal variables to be identified, thus leading to a more refined model of the relationship between autobiographical reasoning valence and psychological functioning. This limitation with the current study could be easily addressed by further research with larger sample sizes.

In summary, the results of the current study extend previous narrative identity research by showing that the valence of autobiographical reasoning found in life story narratives of young adults is associated with a number of cognitive response styles 
(explanatory style, rumination and use of cognitive reappraisal strategies). The results of the current study also suggest that the relationship between autobiographical reasoning valence and psychological functioning may be better understood when additional variables such as cognitive response factors and meaning in life are taken into account. 


\section{Chapter Seven}

\section{Study 3: An investigation into the relationships between autobiographical reasoning, psychological functioning and the visual recall perspective and subjective distance of life story memories}

Across the three studies presented thus far (Studies 1a, 1b, and 2), psychological distress in young adulthood has been shown to be associated with a greater tendency to make negative self-event connections in life story narratives. In two of the three studies (Study 1a and Study 1b), positive psychological functioning, or an absence of psychological distress, has been associated with a greater tendency to make positive self-event connections. Study 2 identified a number of cognitive factors that are associated with young adults' tendency to make negative and positive self-event connections. These findings show that broader patterns of thinking about the world and one's experiences are associated with the way young adults reason about the self in the context of the life story. Study 2 and the current study, Study 3, share the same aim in that both seek to explore key factors that may be associated with the tendency to engage in positive and negative autobiographical reasoning in young adulthood. Study 3 extends this exploration to a slightly different component of the experience of constructing a life story, namely, the phenomenology of autobiographical memories included in the life story.

The justification for examining links between autobiographical reasoning and autobiographical memory phenomenology, the way memories are consciously experienced (Rubin \& Siegler, 2004; Sutin \& Robins, 2007), was two-fold. First, very little research has examined the phenomenology of autobiographical memories included in the life story and to date no research has examined links between phenomenology and autobiographical reasoning. Second, autobiographical memory phenomenology has been shown to be related to psychological functioning (Berntsen \& Rubin, 2006b; D’Argembeau \& Van der Linden, 2004; Kuyken and Howell, 2006; Lemogne et al., 2006). The current study aimed to assess two phenomenological qualities of life story memories that, from a theoretical perspective, may relate to autobiographical reasoning. These two phenomenological qualities include the visual perspective from which life story events are recalled and the subjective distance individuals experience from life story events. Within this broad aim, the current study examined two previously untested associations: 1) whether autobiographical reasoning found in life story narratives was related to the visual perspective from which life story events were recalled and the subjective distance experienced from these events, and 2) whether and how, 
visual perspective and subjective distance interact with autobiographical reasoning valence to predict psychological functioning. Before each of these areas of investigation are outlined, the two phenomenological characteristics - visual perspective and subjective distance - will be discussed.

\section{Visual perspective}

Visual perspective, first described by Nigro and Neisser (1983), is defined as the perspective from which individuals view a remembered event while recalling the experience. Memories recalled from a first-person or 'field perspective' are recalled through the individuals' own eyes, as if they were re-experiencing the event. Memories recalled from a third-person or 'observer perspective' are remembered from an external vantage point, as if the individual were watching the event unfold from the position of a spectator.

From a theoretical perspective, the mental imagery associated with each of the field and observer perspectives alters the relationship between the self and one's environment during the recall process (Libby \& Eibach, 2011). The imagery associated with recalling an event from the field perspective provides the individual with a sense that they are reliving the experience and interacting with their environment in the same way that they did during the actual event (akin to the 'experiential' conception of self; Libby \& Eibach, 2011). Consistent with this notion, first-person memories tend to contain more emotional and sensory information than do observer memories (Nigro \& Neisser, 1983; Sutin \& Robins, 2010). For example, McIsaac and Eich (2004) found that individuals with post-traumatic stress disorder (PTSD) who recalled the traumatic event from the field perspective recalled more emotional reactions, physical sensations and psychological states from the event than did participants who recalled the event from the observer perspective. Although there were no differences in PTSD symptoms across the two groups, participants who recalled the traumatic memory from the field perspective rated the memory as more emotional and anxiety provoking than did participants who recalled the experience from the observer perspective. Similar findings have been detected in relation to non-traumatic memories (Terry \& Horton, 2007-2008).

In contrast, the mental imagery associated with recalling an event from the observer perspective allows individuals to observe and analyse themselves as if from the perspective of another person (Libby \& Eibach, 2011; Sutin \& Robins, 2008). This perspective potentially enables individuals to derive more descriptive and abstract information about the self and to analyse the performance and characteristics of the self in the broader context of the event (the 'conceptual' representation of the self; Wilson \& Ross, 2003; Libby \& Eibach, 2011). 
Consistent with the theoretical views of Libby and Eibach (2011), there is some evidence that the observer perspective increases the focus on descriptive and abstract information about the self during the recall process. In an early study (Frank \& Gilovich, 1989), undergraduate students were asked to engage in a conversation with another participant, and to rate their own behavior along four dimensions: friendliness, dominance, nervousness and talkativeness. At two time points (both at the time of the study and three weeks later), participants rated the extent to which their behaviour during the conversation was caused by personal characteristics of their self (e.g. personality, personal style) or situational factors (e.g. the topic of conversation, the other participant's behavior). Visual recall perspective was manipulated at time two. During the second session, participants were asked to either the recall the conversation from an observer or field perspective. Participants who were asked to recall the event from the observer perspective made more dispositional attributions and fewer situational attributions for their behavior at Time 2 than they did at Time 1. Findings were reversed for the field perspective condition. Participants who were asked to recall the conversation from a field perspective made fewer dispositional and more situational attributions at Time 2 relative to Time 1 . These findings suggest that the observer perspective actively promotes a focus on dispositional attributes (the 'conceptualised' selfrepresentation) in favour of situational factors.

There is also evidence that observer memories are associated with events in which one's knowledge about the self is particularly prominent. Crawley and French (2005) asked undergraduate students to recall two types of childhood events; those that they remembered themselves and those that they had no conscious recollection of but knew had happened (because family members had recounted the event etc.). Participants were also asked to record, when visualising the event, whether the event was recalled using the field or observer perspective. Findings showed that remembered events were more likely to be recalled from a field perspective, whereas events based only on knowledge about the self (e.g. transmitted through parents and close others) were more likely to be recalled from an observer perspective.

In summary, recalling past events from the observer perspective has been associated with an increased tendency to derive abstract and descriptive information about one's selfcharacteristics. Recalling events from the field perspective, on the other hand, has been associated with an increased sense of 'reliving' the event held in memory, in which the internal experiences one had at the time are re-experienced during the process of recall. 
These features of the observer and field recall perspectives will be revisited later in this chapter.

\section{Subjective distance}

Subjective distance is defined as the extent to which an individual feels subjectively close to, or far away from, a recalled past event (Sutin \& Robins, 2007). Memories that are perceived as more distant tend to be associated with a subjective sense that the person the individual was at the time of the recalled event is different to the person they are at the time of recall, with the opposite being true for a subjective sense of closeness (Sutin \& Robins, 2007).

According to Temporal Self-Appraisal theory (Ross \& Wilson, 2002; Wilson \& Ross, 2001; 2003), individuals can influence the way they feel about themselves by altering the way they relate to memories involving 'past selves'. Particularly, positive emotions about the self and a sense of self-improvement can be attained by actively comparing positive aspects of the current self (attributes, skills, strengths) with a past self that did not possess these characteristics (Wilson, Gunn, \& Ross, 2009). Guided by the assumption that individuals are driven to feel positively about themselves, temporal self-appraisal theory proposes that individuals can enhance positive self-regard by retaining a sense of closeness to past selves that are viewed favourably and maximising the distance to past selves that are viewed disparagingly (Wilson \& Ross, 2001; 2003).

There is some evidence that subjective distancing serves to support favourable views of the current self. Wilson and Ross (2001) manipulated participants' subjective sense of distance to a past event by altering the instructions given regarding recall of the event. Participants who were induced to feel close to the remembered event were asked to recall a "time in the recent past, the beginning of this [university] term" while participants who were induced to feel distant from the event were asked to "think all the way back to the beginning of the [university] term" (p. 580). Participants were then asked to evaluate their current self and past self (the self that was represented in the memory) on a number of positive (e.g. social skills, confidence) and negative (e.g. immature, naïve) characteristics. Although the actual time that had elapsed between the event and the time of the study was the same across conditions, participants in the 'close' condition rated their past and present selves similarly, while participants in the 'distant' condition rated their current selves more positively than their past selves. These findings are consistent with the notion that a sense of subjective distance from past events may maintain the individual's perception that they are improving and developing over time (see also Wilson, Gunn, \& Ross, 2009).. 


\section{Visual perspective and psychological distance of life story events}

Very little is known about the visual perspective from which events included in the life story are recalled or the subjective distance individuals experience from life story events. With regard to visual perspective, Kuyken and Howell (2006) asked adolescents aged between 12 and 18 years to describe two self-defining memories and to state whether they recalled the event from an observer or field perspective. Just under $70 \%$ of all self-defining memories were recalled from the field perspective, with the remainder recalled from the observer perspective. This is consistent with earlier findings showing that approximately one-third of autobiographical memories in general are recalled from the observer perspective (Nigro \& Neisser, 1983). In terms of subjective distance, Sutin (2008) asked young adults to write narratives of self-defining memories and rate the sense of subjective distance they experienced in relation to each of these events. The average subjective distance ratings were centered around the mid-point of the scale, suggesting that self-defining memories may not be perceived as particularly close nor distant from the self.

The first aim of the current study was to replicate and extend findings regarding the visual perspective and subjective distance of personally significant events by examining these phenomenological characteristics in the context of events that are specifically included in the life story. With very little prior research on which to base hypotheses, the following two were made: 1) the majority of events included in the life story (high points, low points and turning points) would be recalled from the field perspective and 2) participants would perceive a moderate degree of distance between the memory/the self that is represented in the memory for the event.

\section{Relationships among visual perspective, subjective distance and autobiographical reasoning found in life story narratives}

In light of limited research regarding the phenomenology of life story memories, no research to date has examined whether the visual perspective of, and subjective distance from, life story memoires relate to the process of autobiographical reasoning. From a theoretical perspective, there is reason to expect that such relationships may exist. Narratives of autobiographical memories included in the life story often contain evidence of autobiographical reasoning. As described in earlier chapters, autobiographical reasoning is defined as a process through which individuals make connections between a past experience and an aspect of their current self. 
By definition, autobiographical reasoning involves deriving descriptive and abstract information about the self from a past event. For example,

"A high point in my life was walking across the stage at my high school graduation. I was feeling very proud ... it showed me that yes, if you do work hard and stay focused you will succeed in whatever you do and it shows that I am a committed and very determined person"

In this example, the young adult remembers key details from the event: 'walking across the stage' and 'feeling proud' and through autobiographical reasoning, links the memory for the event to two pieces of abstract information: a life lesson 'if you work hard you can achieve anything' and dispositional characteristics of themselves 'I am a committed and determined person".

The second aim of the current study was to explore key relationships between visual perspective used to recall life story events and autobiographical reasoning. Given the proposed role of the observer perspective in promoting self-evaluative thinking, and prior research showing that the observer perspective is associated with an increased tendency to identify dispositional characteristics and abstract information relating to the self, it is possible that autobiographical reasoning would be more common in narratives of memories that are recalled from the observer perspective than in narratives of memories recalled from the field perspective. In light of no prior research on the relationship between the visual perspective used when recalling life story events and autobiographical reasoning in narratives of these events, the following hypothesis was tentatively made: across all event types (low points, high points and turning points) and controlling for the age of the memory, use of the observer perspective would be positively associated with the amount of autobiographical reasoning found in narratives of these events.

The relationship between subjective distance and autobiographical reasoning may be more complex. Subjective distance from a remembered event is defined by the extent to which the memory represents a self that is similar or different from the person the individual believes they are today. As noted by Pasupathi, Mansour, and Brubaker (2007), autobiographical reasoning can either describe stability or changes in the self over time. Autobiographical reasoning that describes self-stability occurs when a characteristic of the self causes an event or explains why an event has occurred. For example: 
"When I was 18 I left home, moved away and started university ... I was anxious to leave as I knew I would miss my friends and family ... the fact that I moved anyway shows that I am an independent young woman that can make her own choices and knows when to grow up"

Autobiographical reasoning that describes change in the self as the result of an experience can either involve gaining a new insight or the development of personal characteristics or gaining a new awareness of existing characteristics. For example:

"I went to Zimbabwe at the beginning of this year. I was seventeen at the time ...

It was such a new experience to me and I loved every minute of it ... My time in Zimbabwe taught me a lot about what we take for granted here in New Zealand such as power, and safety and I developed a greater sense of culture awareness"

The third aim of the current study was to explore relationships between subjective distance from life story events and autobiographical reasoning. In light of the theoretical notion that memories for events that reflect a changed feature of the self are experienced with greater subjective distance from the current self, it is reasonable to expect that narratives of life story events perceived as distant to the current self will contain more autobiographical reasoning that describes how the self has changed than events perceived as closer to the self. The following prediction was made to explore this previously untested relationship: across all event types (low points, high points and turning points), greater subjective distance from the event would be positively associated with autobiographical reasoning that describes changes in the self.

\section{Relationships among visual perspective and psychological functioning}

As noted earlier, aspects of autobiographical memory phenomenology, such as visual perspective and subjective distance, have also been linked to psychological functioning. Specifically, experiences of negative emotional states (depression and anxiety) have been associated with an increased tendency to recall autobiographical memories from an observer (third-person) perspective. The study by Kuyken and Howell (2006) described above compared the visual perspective used when recalling self-defining memories and autobiographical memories recalled in response to positive and negative cue words by adolescents who met diagnostic criteria for Major Depressive Disorder and non-depressed adolescents. Depressed adolescents were more likely to recall both positive and negative autobiographical memories and self-defining memories from an observer (third-person) 
perspective than non-depressed adolescents. Furthermore, among adolescents who had never been diagnosed with depression, symptoms of depressive states and symptoms were associated with an increased tendency to recall autobiographical memories from an observer perspective.

One theoretical explanation for the link between psychological distress and the observer recall perspective is that the observer perspective reduces emotional engagement with past experiences (Berntsen \& Rubin, 2006; Lemogne et al., 2006). As noted earlier, the imagery associated with recalling an event from the field perspective provides the individual with a sense that they are reliving the experience (Libby \& Eibach, 2011). According to Berntsen and Rubin (2006) and Lemogne et al. (2006), individuals who are experiencing psychological distress demonstrate a preference for the observer recall perspective as they are motivated to avoid the aversive internal experiences associated with many of their life events. While this may serve an avoidance function for experiences of distress in the short term, it may prevent these individuals from reliving past events with positive emotional content.

This theoretical notion is supported by prior work showing that recalling positive experiences from the field perspective allows individuals to engage fully with the positive emotions associated with these enjoyable events, which in turn increase positive affect. Holmes, Coughtry and Conner (2008) asked participants to listen to descriptions of events that had positive outcomes or elements and imagine the event from the field perspective (group 1), observer perspective (group 2) or with a focus on verbal comprehension (control group). Participants also completed a measure of positive affect before and after listening to the event descriptions. Findings showed that participants who imagined the events from the field perspective demonstrated an increase in positive affect over the course of the exercise. In comparison, positive affect decreased in participants who imagined the events from the observer perspective and participants in the verbal condition. A reduced ability to engage with the emotional content of positive events via the field perspective has been conceptualised as a risk factor for psychological distress. In one indirect test of this claim, Bergouignan et al. (2008) found that euthymic individuals in remission from depression (who may remain at higher risk of relapse than never-depressed individuals) were less likely to recall positive experiences from a field perspective than were never-depressed controls.

The fourth aim of the current study was to examine this theoretical link between the observer perspective and psychological functioning in the context of positive and negative events from the life story. Following the theoretical notion that the observer perspective may be one key strategy adopted by psychologically distressed individuals to reduce emotional 
engagement with difficult experiences, I predicted that young adults who recalled low points, high points and turning points from the life story from the observer perspective would experience more negative emotional states (symptoms of depression, anxiety and stress) than young adults who recalled these events from the field perspective.

An alternative theoretical explanation for the relationship between the observer perspective and psychological distress is that the increased focus on self-evaluation afforded by the observer perspective increases the salience of negative self-perceptions that are typically held by psychologically distressed individuals. In the case of depression, beliefs about the self tend to be negative in nature (e.g. "I'm useless") and maintained by ruminative thoughts about the self and the cause of one's distress (Kuyken \& Howell, 2006). The observer perspective, given that it promotes self-evaluative thinking and an increased tendency to identify dispositional and abstract information about the self, increases the salience of negative thoughts and self-beliefs that distressed individuals hold about themselves. In support of this claim, Kuyken and Moulds (2009) asked depressed adults to recall autobiographical memories in response to positive, negative and neutral cue words and to indicate the visual perspective from which the event was recalled. Participants also completed the self-evaluation subscale of the Depressed States Checklist (DSC; Teasdale \&Cox, 2001), which contains 14 negative self-evaluations associated with dysphoria. Participants who endorsed more negative self-evaluations were more likely to recall autobiographical memories from an observer perspective. While this provides indirect evidence of an association between negative self-evaluation and the observer perspective, it should be noted that these authors did not control for depressive symptoms nor does the cross-sectional design allow any statements regarding the directionality of the relationship to be made.

Similar to the findings of Kuyken and Moulds (2009), relationships have also been established between the observer perspective and symptoms of anxiety, where negative selfperceptions are also common. Sutin and Robins (2010) found that undergraduate students who scored highly on a measure of trait anxiety were more likely to adopt a general thirdperson retrieval style and recall specific self-defining memories from the observer perspective than students scoring lower on trait-anxiety. Similarly, D'Argembeau, Van der Linden, d'Acrement, and Mayers (2006) asked participants with clinical diagnoses of social anxiety and control participants with no history of anxiety disorders to recall specific autobiographical memories of positive and negative events involving social and non-social situations. There were no differences in the use of the observer perspective for non-social 
events between socially anxious and non-anxious individuals. Socially anxious individuals were, however, more likely than non-anxious controls to recall social situations from an observer perspective. Interpreting these findings in relation to the self-evaluation hypothesis is consistent with cognitive models of social anxiety, which suggest that socially anxious individuals experience a heightened sense of focus and are susceptible to making negative judgements about themselves and their performance during social situations (Rapee \& Heinberg, 1997).

The fifth aim of the current study therefore, was to examine this theoretical notion in the context of memories included in the life story and autobiographical reasoning found in narratives of these events. As described in earlier chapters, autobiographical reasoning can either describe the self in positive, negative or neutral/mixed ways. For example, positive autobiographical reasoning may describe positive characteristics or qualities held by the current self:

"When I was 17 I made the decision to go to boarding school in another city away from home for my final year of school ... I have never been put under pressure to make a decision about something so important before ... I became more confident in myself and independent in a sense"

Negatively valenced autobiographical reasoning on the other hand, describes negative features or qualities of the current self:

"My mum moved to [Country], when I was 12 . I felt extremely abandoned ... and I still struggle to form close relationships with others to this day"

If the observer perspective and psychological distress are accounted for by the increased salience of negative self-evaluations, then we would expect to find a relationship between use of the observer perspective to recall life story events and the amount of negative autobiographical reasoning found in narratives of these events in young adults experiencing higher levels of depression. To examine this possibility, I hypothesised that among young adults with higher levels of depressive symptoms, use of the observer perspective to recall life story events would be associated with a greater amount of autobiographical reasoning that describes the self in negative ways in narratives of these events. This same relationship between the observer perspective and negative autobiographical reasoning was not expected to be present for young adults with lower levels of depressive symptoms who were thus not likely to be making many negative self-event connections. 


\section{The relationship between subjective distance and psychological functioning}

The link between the subjective distance of autobiographical memories and psychological functioning is more tenuous. There is evidence that psychologically healthy individuals demonstrate a tendency to perceive positive events in general as subjectively closer than negative events (D'Argembeau \& Van der Linden, 2004). Furthermore, and as outlined above, psychologically distancing oneself from past experiences may serve a selfenhancement function (Wilson \& Ross, 2001). That is, individuals may perceive events that portray the self in a negative way as more psychologically distant, and experience psychological closeness to an event that portrays the self in a positive or favourable way. This theoretical assumption stems from evidence that psychologically healthy individuals, at least in Western cultures, are motivated to think about themselves in positive ways (Taylor \& Brown, 1988).

Although it has not been directly tested, there is an assumption that individuals who experience poor psychological functioning do not demonstrate these same self-enhancing tendencies. For example, Ross and Wilson (2002) asked undergraduate students to record either their best or worst grade from the previous academic term, record how distant or close that university course felt to them, and complete a measure of self-esteem. Findings showed that young adults who scored high on self-esteem perceived less subjective distance to their best grade than they did their worst grade. Young adults who scored low on self-esteem did not show this bias. That is, young adults with low self-esteem felt just as close to worst grades as they did to best grades. This effect was replicated in a second study, which showed that young adults who scored highly on the measure of self-esteem felt closer to past selves that were evaluated in a positive way and further away from past selves that were evaluated in a negative way. Young adults who scored low on the measure of self-esteem did not demonstrate this bias, that is, past selves were perceived as equally distant regardless of whether the self was evaluated positively or negatively.

The sixth and final aim of the current study was to examine key relationships between psychological distance, the valence of autobiographical reasoning and psychological functioning. In light of the findings of Ross and Wilson (2002), several tentative hypotheses were made. The first prediction was that negative emotional states would be positively associated with perceived distance to high points from the life story and inversely associated with perceived distance from low points from the life story. That is, young adults who felt subjectively close to high points, and subjectively more distant to low points would experience fewer negative emotional states (symptoms of depression, anxiety and stress) than 
young adults who felt subjectively close to low points, and more distant from high points. As turning points can be negative or positive in nature, the question of whether subjective distance to turning points would be associated with negative emotional states was left open.

The second prediction, made in accordance with the self-enhancement hypothesis, was that young adults with lower levels of depressive symptoms would experience greater distance from past experiences that led to positive changes in the self (as measured by autobiographical reasoning that describes positive change). Conversely, this relationship would not be present for young adults who experienced higher levels of depression and who would thus be less likely to make positive change connections overall.

\section{Method}

\section{Participants}

The method used to recruit participants was identical to that adopted in Chapter Four. Participants were 103 undergraduate students (68\% female) enrolled in an introductory psychology course at Victoria University of Wellington, New Zealand. Participants received credit towards a course requirement of participation in research. Ages ranged from 18 to 25 years $(M=19.5$ years, $S D=1.2$ years $)$. Eighty-three percent of participants identified as New Zealand European, 10 percent as New Zealand Maori, 5 percent as Asian, 4 percent as Pasifika, and 11 percent as other (one in ten participants identified with more than one ethnic background).

\section{Overview of Procedure}

The study was conducted over one 60-minute session. Each session took place in a psychology laboratory; participants (with numbers ranging between one and four per session) were seated at individual desks, separated by dividers. At the start of the first session, participants read a summary of the nature and purpose of the study and provided consent. In the 60-minute session, participants completed the Depression Anxiety Stress Scale (DASS; Lovibond \& Lovibond, 1995) and an autobiographical narrative task through an online survey created in Qualtrics (Qualtrics Labs Inc., Provo, UT). Immediately after completing each written narrative, participants answered a series of questions examining the centrality of the event, the visual perspective from which the event was recalled when writing the narrative and participants' sense of subjective distance from the event. The order in which participants completed the DASS and the autobiographical narrative task was counterbalanced. 


\section{Tasks and measures}

Symptoms of psychological distress. Depression Anxiety Stress Scale (DASS;

Lovibond \& Lovibond, 1995). This self-report questionnaire measures three related negative emotional states: depression, anxiety and stress, each on a 14-item subscale. Participants rate on a four-point scale how much each symptom applied to them over the past week $(0=\mathrm{Did}$ not apply to me at all, $3=$ Applied to me very much, or most of the time). Consistent with previous research (Crawford \& Henry, 2003), a total DASS score was calculated by summing each of the subscales. Internal consistency for the DASS total score within the current sample was .97.

Visual perspective and Subjective distance. Memory Experiences Questionnaire (MEQ; Sutin\& Robins, 2007). Two subscales from the MEQ were used to measure visual perspective (6 items) and subjective distance (6 items). Participants rated on a 5-point scale ( $1=$ strongly disagree, $5=$ strongly agree $)$ how much they agree with each item. The visual perspective subscale comprised three items that measured the extent to which the memory was recalled through the field perspective and three items that measured the extent to which the memory was recalled from the observer perspective. A total 'visual perspective' score was calculated by summing the observer and (reverse-scored) field items. The subjective distance subscale comprised three items that measured the extent to which the person they were in the experience was perceived to be the same and three items that measured the extent to which the person they were in the experience was perceived to be different. A total 'subjective distance' score was calculated by summing the 'same' and (reverse-scored) 'different' items.

Autobiographical narrative task. Life story narratives were attained through an autobiographical narrative task modified from McAdams' guided autobiography and widely used in previous research (McAdams, 2006; available at http://www.sesp.northwestern.edu/foley/instruments/guided/; see also McLean \& Breen, 2009; McLean \&Lilgendahl, 2008). Participants were asked to provide written narratives of a high point, low point and turning point from their life story. High points were defined as "moments or episodes in a person's life in which he or she feels a sense of happiness, joy, excitement, contentment or some other positive emotion". Low points were defined as "moments or episodes in a person's life in which he or she feels extremely negative emotions such as despair, disillusionment, guilt, shame etc." Turning points were defined as "episodes through which a person undergoes substantial transition or change with respect to their understanding of themselves". 
Participants were asked to write about each event in some detail, and to consider the following in their descriptions: how old they were when the event occurred, what happened in the event, who was involved in the event, what they were thinking/feeling/wanting in the event, why they think that this is an important event in their life story and what the event says about who they are. The order in which participants wrote about their high point, low point, and turning point was counter-balanced.

\section{Narrative coding}

\section{Self-event connections.}

Following McLean and Fournier (2008), self-event connections were coded as any statements where participants explicitly linked an aspect of the narrated event to their sense of self. Consistent with the procedure outlined in the previous three studies (Study 1a, 1b, and Study 2), all written narratives were coded for self-event connections by the first author. Twenty-eight percent of the narratives were coded by an independent coder who was blind to the hypotheses of the study. Consistent with the approach taken in the previous three studies (Study 1a, 1b, and Study 2), the number of self-event connections was calculated on a per narrative basis and inter-rater agreement for the total number of connections per narrative was kappa .83 .

Consistent with the procedure outlined in the previous three studies (Study 1a, 1b, and Study 2), self-event connections were also coded for valence. Self-event connections were coded as positive when the statement referred to a positive characteristic of the self, mentioned personal growth, or denoted a positive evaluation of the self (e.g., "This event made me stronger"). Self-event connections were coded as negative when the statement referenced a negative characteristic of the self, or denoted a negative evaluation of the self (e.g., "this event is one of the main causes for the long term depression that I experience"). Self-event connections were coded as neutral when there was no evaluation of the self, or the connection was not clearly positive or negative (e.g., "this experience changed how I think about the world"). Self-event connections were coded as mixed when the connection involved elements of both positive and negative meaning. These were often situations where participants had learnt something (and thus could be considered an experience involving growth), but where the lesson learnt had negative connotations (e.g. "This experience taught me about the harsh realities of life").

Self-event connections were only recorded as positive or negative if: the selfcharacteristic highlighted was explicitly referred to as positive or negative by the participant (e.g., "It was a great experience because it taught me how to be independent" - positive; "It 
has helped me over the last year, but in a negative way" - negative), or if it satisfied common cultural understandings of desirable and undesirable characteristics (e.g., "I gained a lot of self-confidence" - positive, and "This [event] has made me more paranoid" - negative). Self-event connections that were identified by both coders ( $90 \%$ of connections identified by the first coder) were compared for valence. Inter-rater agreement for the valence of selfevent connections was kappa .80 .

Self-event connections were also coded as either a change connection or a stability connection. Self-event connections were coded as a change connection when the link between event and self clearly outlined how the event had caused a change in terms of the characteristics or qualities of the individual (e.g. I was a secluded person and minded my own business but now I am more outgoing). Self-event connections were coded as a stability connection when the link between event and self clearly outlined a continuation of the individuals qualities and characteristics from the past event to the current moment (e.g. [this event] shows that I am a committed and determined person). Inter-rater agreement was kappa .84 for change/stability connections.

\section{Results}

\section{Descriptive statistics}

Means and standard deviations for the measure of visual perspective and subjective distance are reported for each event type in Table 7.1. The average subjective distance rating was close to the mid-point (on the $1-5$ point scale) for all three event types (high, low and turning points). This finding is consistent with the hypothesis that participants would perceive a moderate degree of distance between the memory/the self that is represented in the memory for the event.

Of note, memories for life story high points were appraised as subjectively closer than memories for life story low points $(t(102)=7.72, p<.05)$, and memories of turning points $(t$ $(102)=6.64, p>.05)$. There were no significant differences in the subjective distance of low and turning points from the life story $(t(102)=1.49, p>.05)$.

Although the differences were small, high points were more likely to be recalled from the field perspective than were low points $(t(102)=3.15, p>.05)$ and turning points $(t(102)$ $=2.27, p>.05$ ). On average however, all event types (high points, low points and turning points) scored below the mid-point on the field-observer scale, suggesting a greater overall tendency to recall these events from the field perspective.

Participants' visual perspective ratings for each memory were then grouped according to whether they favoured the observer perspective (scores above the mid-point of 2.5 on the 
1-5 scale), the field perspective (scores below the mid-point of 2.5) or neither perspective over the other (scores equalling the mid-point of 2.5). Almost all high points (98\%) were recalled from the field perspective, in addition to the majority of low point events (91\%) and turning point events (93\%). In total, $n=18$ out of $n=309$ life events narrated by participants during the study (6\% in total) were recalled from the observer perspective. These findings supported the hypothesis that the majority of events included in the life story (high points, low points and turning points) would be recalled from the field perspective.

\section{Table 7.1}

Means, standard deviations and ranges for visual perspective and subjective distance ratings for each memory type

\begin{tabular}{lcccc}
\hline & \multicolumn{2}{c}{ Visual perspective } & \multicolumn{2}{c}{ Subjective distance } \\
\cline { 2 - 5 } & $M$ & $S D$ & $M$ & $S D$ \\
\hline High points & 2.1 & .71 & 2.1 & .73 \\
Low points & 2.3 & .89 & 3.0 & .93 \\
Turning points & 2.3 & .75 & 2.8 & .86 \\
\hline
\end{tabular}

\section{Relationship between visual perspective and negative emotional states}

The majority $(n=84,82 \%)$ of young adults in the current sample recalled all three life story events from the field perspective. A small number $(\mathrm{n}=13,13 \%)$ of young adults recalled one or more life story event from the observer perspective. Of these participants, nine $(9 \%)$ recalled one out of three events from the observer perspective, three participants recalled two of three events from the observer perspective and just one participant recalled all three events from the observer perspective. Although the intention was to examine differences in psychological distress by use of the observer perspective across different event types (low points, high points etc.), a decision was made to collapse observer memories across all event types due to the low number of events recalled from the observer perspective.

To examine whether use of the observer perspective was associated with symptoms of psychological distress, a one-way ANOVA examined whether young adults who recalled at least one life story event from the observer perspective $(n=13)$ experienced more negative emotional states than young adults who recalled all life story events from the field perspective $(n=84)$. Participants who did not recall any of the three life story events from 
the observer or field perspective $(n=6)$ were excluded from the analysis. Although in the expected direction, no significant effect for observer perspective was found, $F(1,95)=$ $1.77, p>.05$. That is, although participants who recalled at least one life story event from the observer perspective experienced higher levels of negative emotional states $(M=28.9, S D=$ 27.6) than participants who did not recall any events from the observer perspective $(M=21.4$, $S D=17.2$ ), this difference was non-significant. A series of one-way ANOVAs then examined whether differences existed for each of the distress states separately (depression, anxiety and stress). Again, no significant differences in symptoms of depression, anxiety or stress were detected between the two groups $(F s=.52$ to $2.30, p s>.05)$. Thus the hypothesis that psychological distress would be associated with the observer recall perspective was not supported.

\section{Relationship between visual perspective and autobiographical reasoning}

To test the hypothesis that use of the observer perspective would be positively associated with the amount of autobiographical reasoning found in narratives of these events, Pearson correlations tested the associations between visual perspective and number of selfevent connections in narratives of life story low, high and turning points. Across all event types, there were no significant relationships between the visual perspective used to recall the event and the number of self-event connections found in narratives of these experiences ( $r s=$ .11 to $.17, p s>.05)$. Thus no support was found for the hypothesis that autobiographical reasoning would occur more frequently in narratives of memories recalled from the observer perspective.

\section{Relationship between visual perspective, autobiographical reasoning and negative emotional states}

Pearson correlations then tested the hypothesis that among young adults with higher levels of depressive symptoms, use of the observer perspective to recall life story events would be associated with a greater amount of autobiographical reasoning that describes the self in negative ways. The first step in this analysis was to categorise participants into groups depending on their level of reported depression symptoms. Following the DASS manual (Lovibond \& Lovibond, 1995), participants' scores on each of the DASS subscales were first categorised according to severity. Depression scores were initially categorised as normal $($ DASS score $=0-9)$, mild $($ DASS score $=10-12)$, moderate $($ DASS score $=13-20)$, severe $($ DASS score $=21-27)$ and extremely severe (DASS score $=28+$ ). As proportionately few participants scored within each of the clinical ranges, the mild, moderate, severe and extremely severe groups were combined to form a broader 'clinical' group for each of the 
three emotional states. Thus participants were categorised into two groups for Depression ('normal', scores $=0-9$; 'elevated', scores $=10+$ ). Due to the small number of events recalled from the observer perspective, participants' ratings on the 5-point visual perspective subscale were used for the following correlations. As few participants rated their visual perspective above the mid-point (i.e. towards the observer perspective), higher ratings reflect not only a stronger observer perspective, but also a weaker field perspective.

Table 7.2 presents the correlations between the visual perspective used to recall high points, low points and turning points, and the number of negative self-event connections made in narratives of these events for individuals in the 'normal' and 'elevated' symptoms of depression groups. For individuals in the normal category, visual perspective was unrelated to the number of negative self-event connections made in narratives of high, low or turning points $(r s=-.01$ to $.11, p s>.05)$. For young adults experiencing elevated levels of depression however, the number of negative self-event connections made in narratives of low points was associated with an increased tendency to recall these events from the observer perspective (or a decreased tendency to recall events from the field perspective) ( $r s=-.08$ to $.01, p>.05)$. Negative self-event connections in narratives of high and turning points were unrelated to the visual perspective from which these events were recalled ( $r s=-.08$ to $-.01, p s$ $>.05$ ) for young adults in the 'elevated' category.

Table 7.2

Correlations between the number of negative self-event connections made in narratives of low points and use of the observer recall perspective

\begin{tabular}{ccc}
\hline \multirow{2}{*}{$\begin{array}{c}\text { Number of negative } \\
\text { connections }\end{array}$} & \multicolumn{2}{c}{ Use of observer recall perspective } \\
\cline { 2 - 3 } & $\begin{array}{c}\text { Normal' depression } \\
\text { symptoms }\end{array}$ & $\begin{array}{c}\text { 'Elevated' depression } \\
\text { symptoms }\end{array}$ \\
\hline Turning points & -.11 & -.01 \\
Low points & -.01 & $.44 *$ \\
High points & .01 & -.08 \\
\hline$*_{p}<.05, * * p<.01$ & &
\end{tabular}




\section{Relationship between subjective distance and negative emotional states}

To test the prediction that negative emotional states would be positively associated with perceived distance to high points from the life story and inversely associated with perceived distance from low points from the life story, Pearson correlations examined these key relationships. Negative emotional states were significantly and positively associated with perceived distance from life story high points $(r=.24, p<.05)$. Thus, young adults who perceived greater distance between their current self and the self represented in high points from the life story experienced more negative emotional states than young adults who felt closer to these positive experiences. There were no significant relationships between perceived distance from low points and turning points from the life story and negative emotional states ( $r s=-.09$ to $-.06, p s>.05$ ).

\section{Relationship between subjective distance and autobiographical reasoning}

Partial correlations were then performed to test the predicted associations between subjective distance and the amount of autobiographical reasoning in narratives of life story low, high and turning points that described a change in the self. Across all event types, there were no significant relationships between the subjective distance between the event and the amount of autobiographical reasoning describing self-change in narratives of these experiences ( $r s=-.10$ to $.04, p s>.05$ ). Thus contrary to predictions, there was no evidence that autobiographical reasoning describing self-change occurred more frequently in narratives of memories that were appraised as more distant to the current self.

\section{Relationship between subjective distance, autobiographical reasoning and negative emotional states}

Lastly, Pearson correlations were performed to test the hypothesis that subjective distance from high points, low points and turning points from the life story would be related to the number of self-event connections depicting positive change made in narratives of these events. These correlations were examined for young adults in the 'normal' depression range and the 'elevated' depression range separately. For individuals in the 'normal' category, subjective distance from high, low or turning points was unrelated to the number of self-event connections depicting positive self-change made in narratives of these experiences $(r s=-.05$ to $.18, p s>.05)$. For young adults in the 'elevated' depression range, subjective distance from high, low or turning points was also unrelated to the number of self-event connections depicting positive self-change ( $r s=-.10$ to $-.04, p s>.05$ ). Thus no support was found for the hypothesis that subjective distance from high points, low points and turning points from the 
life story would be related to the number of self-event connections depicting positive change made in narratives of these events.

\section{Discussion}

Very little research has examined the phenomenology of autobiographical memories included in the life story. The broad aims of the current study were to 1) extend life story research by assessing two aspects of memory phenomenology; visual perspective and subjective distance in memories for events included in the life story, and 2) explore some of the key relationships between these phenomenological characteristics, autobiographical reasoning (amount and valence) and psychological functioning.

As expected, the majority of life story events narrated by young adults in the current study were recalled from the field perspective. The proportion of events recalled from the field perspective was, however, much higher than previous assessments of self-defining memories and general autobiographical memories elicited via the Autobiographical Memory Test (Kuyken \& Howell, 2006; Sutin, 2008). Young adults reported an average degree of subjective distance from life story events which, consistent with prior work, suggests that they do not feel particularly close or distant from these experiences (Sutin, 2008). On average, young adults in the current study perceived greater distance from low point and turning point events from the life story than they did from high points. Findings relating to visual perspective, followed by key findings related to subjective distance will now be discussed.

\section{Visual perspective}

Unexpectedly, there was no evidence of a relationship between use of the observer perspective to recall events from the life story and negative emotional states. That is, young adults who recalled one or more life story events from the observer perspective did not experience significantly higher levels of psychological distress (as measured by total DASS score and in relation to each of the distress types; depression, anxiety and stress) than young adults who solely recalled events from the field perspective.

One explanation for these non-significant findings is that the relationship between the observer perspective and psychological distress depends on a magnitude of distress symptoms that were not present among participants in the current study. Much of the previous work on the relationship between the observer perspective and psychological distress has been with individuals with clinical diagnoses such as Major Depressive Disorder (Kuyken\& Howell, 2006). Only a small proportion of young adults who participated in the 
current study experienced clinical levels of psychological distress, which may not have allowed significant differences to be detected. The findings of Kuyken and Howell (2006) challenge this view, however, as even among young adults whose symptoms were within a 'normal' range, an increase in symptoms of depression were associated with an increased use of the observer perspective.

A more likely explanation for the non-significant relationship between the observer perspective and psychological distress is that the overwhelming proportion of life story events reported in the current study were recalled from the field perspective. This also unexpected finding may be associated with a number of methodological issues. In the current study, participants' use of the observer and field recall perspective was measured by the visual perspective subscale of the Memory Experiences Questionnaire (Sutin \& Robins, 2007) which was completed after participants had written narratives of life events. There are two possible problems with using this scale in relation to life story events, particularly when it is administered after participants had written narratives of these experiences. First, the wording of some of the items on the visual perspective sub-scale may be misconstrued by participants.

A number of items in the visual perspective subscale of the MEQ use the terms "perspective" and "through my own eyes" to assess the visual perspective adopted when imagining the remembered event (Sutin \& Robins, 2007). Example items include "I see the experience in the memory through my own eyes" and "When I visualize this memory, I clearly see this event from my own perspective" (Sutin \& Robins, 2007). Another definition of perspective, however, relates to the state of one's knowledge or perceptions one holds in relation to an experience e.g. "I can see it now from their perspective", "In my eyes, I was in the right all along". Constructing narratives about life experiences, which often include one's thoughts and reflections on the event, may have primed participants to think about these events in more abstract terms thus increasing the likelihood that they would interpret the terms 'perspective' and 'in my own eyes' in the latter way. That is, participants may have been more likely to interpret 'perspective' as denoted 'from my point of view'. An alternative method of eliciting visual perspective used in prior research involves describing each of the observer and field perspectives and asking participants to state which perspective they use when recalling the event. This method has been successfully used in prior work (Crawley \& French, 2005; Frank \& Gilovich, 1989; Libby \& Eibach, 2011) and may be preferable in situations where post-event processing (e.g. the construction of life story narratives) has recently occurred. 
Assessing memory phenomenology after the construction of life story narratives may not be a suitable or reliable measure of the true phenomenological characteristics of these memories for another reason. Most life story narratives reference a specific past event but, through the process of narration, often incorporate details about what was happening before the event, details about what impact the event had on the person subsequent to the event and often, as observed through autobiographical reasoning, what the impact on the event is on the individual in the present. This combination of past and present details can be observed in the following example:

"A turning point in my life would have to be when I met my friend [name] when I was 16 years old. Prior to this I was really struggling with my faith and reconciling traditional perceptions of Catholicism with what I thought was morally right. [Name] showed me was it means to live out in action that Catholic faith. Through his encouragement and cheerfulness he revitalised my spirituality ... I now feel extremely connected to the people in my faith, more confident in myself and more enthusiastic to change what I believe is detrimental to the Church"

In this example, the memory for the past event (i.e. meeting a friend) was blended with details about what was happening before (struggling with faith), what happened after the event (her spirituality was revitalised) and what the lasting impact of the event is now (connected to faith, more confident and enthusiastic). This combination of details and points in time may obscure the visual perspective from which the memory for the original event (e.g. "the day I met my friend) would be recalled and/or prevent participants from clearly seeing the event from any one perspective. Future research could address this possible methodological limitation by altering the order in which the autobiographical narrative task and visual perspective assessment are completed. That is, first participants would be asked to elicit memories for events that would be included in the life story, imagine the event and record the visual perspective from which it is recalled, and then construct a narrative of the experience.

Findings with respect to the relationship between the observer perspective and autobiographical reasoning were mixed. No significant associations were found between use of the observer perspective and the overall amount of autobiographical reasoning found in narratives of life story high, low and turning points. When analysed in the context of levels psychological distress (depression) however, an interesting trend emerged. For young adults 
experiencing elevated levels of depression, the more negative self-event connections they made in narratives of low points the more likely they were to recall these events from the observer perspective. No such relationship existed for young adults who were not experiencing depressive symptoms.

These findings are consistent with the theoretical notion that depressed individuals may, in part, experience higher levels of distress because the observer perspective is associated with the processes of self-evaluation and self-description which, in depressed individuals, tends to be of a negative nature (D'Argembeau, Van der Linden, d'Acrement \& Mayers, 2006; Kuyken \& Moulds, 2009). The fact that this relationship existed in the context of low points from the life story but not high points or turning points is not surprising given that in distressed individuals, negative information about the self is more readily activated in relation to negative events than events of a more positive nature (Riskind \& Alloy, 2006).

The lack of a significant association between use of the observer perspective and the overall amount of autobiographical reasoning found in life story narratives may reflect differences in the concepts of autobiographical reasoning and self-evaluation/description. In one study showing that inducing the observer recall perspective led to an increase in selfdescriptors (Frank \& Gilovich, 1989), participants were specifically asked to report attributions (dispositional or situational) for their behaviour. Autobiographical reasoning encompasses a wider range of self-descriptors than dispositional traits, however. That is, autobiographical reasoning can also be employed to describe an individual's goals, achievements, and qualities of relationships they have with others (McLean \& Fournier, 2008). One possibility is that the observer perspective may be associated with an increased focus on internal characteristics of the self (dispositions) but unrelated to aspects of the self that are centered outside the individual (e.g. a close bond with a family member).

In discussing these findings, it is important to remember that a very small number $(6 \%)$ of life story events narrated by participants in the current study were recalled from the observer perspective. This limitation is likely to have significantly reduced the opportunity for significant differences to be detected. Future research with a larger sample of events recalled from the observer perspective is needed to delineate whether the small number of observer memories in the current study contributed to the non-significant findings. 


\section{Subjective distance}

Overall, young adults in the current study rated high point events from the life story as subjectively closer than low point and turning point events. The extent to which high points were perceived as subjectively distant however, was significantly associated with psychological distress. That is, young adults who felt subjectively closer to the memory of high points from the life story experienced fewer negative emotional states than young adults who felt subjectively more distant from these memories. This finding is consistent with earlier research showing that psychologically healthy individuals typically experience less subjective distance from positive events than they do from negative events (D'Argembeau\& Van der Linden, 2004; Wilson \& Ross, 2001).

There was no evidence that the amount of autobiographical reasoning describing change in the self found in life story narratives was associated with the subjective distance participants experienced from these events. Furthermore, there was no evidence that life story events which resulted in positive self-change (as described by autobiographical reasoning), were viewed as more distant than events that did not result in positive selfchange. These non-significant findings were surprising in light of previous theoretical and empirical work showing that a) greater subjective distance from past events is observed when the individual perceives the person they are now is different to the person they were during the remembered event and b) psychologically well-functioning individuals may perceive greater distance from events in which positive self-change has occurred to preserve the view that they are improving over time (Libby, Eibach, \& Gilovich, 2005).

The absence of the hypothesised relationships between subjective distance and autobiographical reasoning describing self-change may also be explained in terms of the methodological constraints and qualities of life story narratives mentioned earlier. As with the measure of visual perspective, participants completed the subjective distance subscale of the MEQ after constructing narratives of their experiences. As discussed earlier, narratives of life story events often blend details of the past event with details about what was happening before the event, what happened after the event, and what the lasting impact the event has for the individual now. This focus on both the past and present within life story narratives means that both past and current selves are represented. This combination of past and present focus is likely to have altered the way participants relate to items measuring subjective distance such as "I don't have much in common with the person in the memory" as 'the person in the memory' includes both the past and present self. Again, changing the order in which 
participants complete the subjective distance measure and autobiographical narrative task may address this concern in future research.

In summary, the current study has highlighted a number of methodological concerns that require consideration when examining the phenomenology of autobiographical memories included in the life story. Despite these limitations, the current study also highlighted a number of interesting findings. First, for young adults who were experiencing clinically significant symptoms of depression, the recall of life story events from the observer perspective was associated with an increase in autobiographical reasoning that described the self in negative ways. Second, young adults who felt subjectively more distant from life story high points experienced higher levels of negative emotional states than young adults who perceived less distance from life story high points. These findings suggest that the phenomenology of autobiographical memories included in the life story may be a relevant construct to future investigations into the relationship between life story development and psychological functioning. 


\section{Chapter Eight}

\section{General Discussion}

This aim of this thesis was to contribute to the growing body of work examining the relationship between autobiographical reasoning and psychological functioning. Although not directly examined, this thesis was based on the theoretical premise that autobiographical reasoning is the primary process through which the life story is developed (Habermas \& Bluck, 2000). The current thesis extended prior work by introducing the concept of autobiographical reasoning valence as measured by positive, negative, neutral and mixed valence self-event connections. Over four main studies, a number of key questions regarding the relationship between autobiographical reasoning and psychological functioning in young adulthood were addressed.

The first study explored the question of whether the relationship between autobiographical reasoning and psychological functioning was altered by the extent to which young adults linked past events to positive or negative characteristics of the self. The second study addressed the question of whether autobiographical reasoning predicted changes in psychological functioning over time. Although not a true test of causality and constrained by the small sample size, this pilot study illustrated how the use of a longitudinal design may be used in future research to assess how these two factors might be related over time. The third and fourth studies aimed to explore relationships between autobiographical reasoning valence and three sets of additional psychological constructs: cognitive response styles; meaning in life; and autobiographical memory phenomenology. These factors were selected on the basis of theoretical evidence suggesting they may be related to the process of autobiographical reasoning. Inter-relationships between these factors (cognitive response styles; meaning in life; and autobiographical memory phenomenology), autobiographical reasoning valence, and psychological functioning were also explored.

\section{Key findings}

The studies presented in this thesis show that the valence of autobiographical reasoning found in life story narratives constructed by young adults predicts psychological functioning in this population group. Table 8.1 shows associations between the overall number of positive and negative self-event connections found in life story narratives and indicators of psychological functioning across the three main studies (Study 1a, Study 2 and Study 3). Across two studies (Study 1 and Study 2), young adults who made a greater number of positive self-event connections in narratives of life story events experienced better 
psychological well-being and were more satisfied with their lives than young adults who made fewer positive self-event connections. In two out of three studies (Study 1 and Study 3), young adults who made more positive self-event connections in life story narratives also experienced fewer symptoms of psychological distress than young adults who made fewer positive connections ${ }^{10}$. Conversely, in all three main studies, young adults who made a greater number of negative self-event connections in life story narratives experienced more symptoms of psychological distress relative to young adults who made fewer negative selfevent connections.

Table 8.1

Correlations between positive and negative self-event connections and psychological functioning across three studies

\begin{tabular}{|c|c|c|c|c|c|c|}
\hline \multirow[b]{2}{*}{ Study } & \multicolumn{2}{|c|}{$\begin{array}{c}\text { Psychological distress } \\
\text { (DASS) }\end{array}$} & \multicolumn{2}{|c|}{$\begin{array}{l}\text { Psychological well-being } \\
\text { (RYFF scales) }\end{array}$} & \multicolumn{2}{|c|}{ Satisfaction with life } \\
\hline & $\begin{array}{c}\text { \# of } \\
\text { positive } \\
\text { connections }\end{array}$ & $\begin{array}{c}\text { \# of } \\
\text { negative } \\
\text { connections }\end{array}$ & $\begin{array}{c}\text { \# of } \\
\text { positive } \\
\text { connections }\end{array}$ & $\begin{array}{c}\text { \# of } \\
\text { negative } \\
\text { connections }\end{array}$ & $\begin{array}{c}\text { \# of } \\
\text { positive } \\
\text { connections }\end{array}$ & $\begin{array}{c}\text { \# of } \\
\text { negative } \\
\text { connections }\end{array}$ \\
\hline 1 & $-.28 * *$ & $.39 * *$ & $.31 * *$ & $-.40 * *$ & & \\
\hline 2 & -.08 & $.23 * *$ & & & $.16^{*}$ & $-.33 * *$ \\
\hline 3 & $-.29 * *$ & $.22 *$ & & & & \\
\hline
\end{tabular}

Earlier theoretical and limited empirical work suggested that the process of autobiographical reasoning may reflect positive psychological functioning in young adulthood because of its proposed role in the development of narrative identity (McAdams, 2001; McLean, 2005; 2008; McLean \& Breen, 2009; McLean \& Pratt, 2006). The findings of Study 1a (presented in Chapter Four) showed that more autobiographical reasoning (as measured by the number of self-event connections) and more complex forms of autobiographical reasoning (as measured by sophistication of meaning ratings) found in young adults' life story narratives were unrelated to psychological functioning. Rather, the

10 In Chapter Five, the significantly lower DASS scores recorded by participants in Study 2 relative to participants in Study 1a was offered as a possible explanation for the non-significant relationship between the number of positive self-event connections and DASS scores. The mean DASS score recorded by participants in Study 2 was not significantly different to the mean DASS score recorded in Study 3 (in which a significant association between positive self-event connections and DASS scores was found). As such, the absence of a relationship between positive connections and DASS scores in Study 2 remains unexplained. 
valence of self-event connections found in young adults' life story narratives predicted whether the amount and/or complexity of autobiographical reasoning was associated with positive or poor psychological functioning. These results highlight the importance of autobiographical reasoning valence in the relationship between life story development and psychological functioning during the developmental period of young adulthood.

Due to the wide scope of questions and issues examined in the remainder of this thesis, key findings are presented in two sections. First, findings regarding the relationship between life story development and positive psychological functioning are discussed, followed by findings regarding the relationship between life story development and poor psychological functioning.

\section{The development of the life story and positive psychological functioning}

As the current thesis coded autobiographical reasoning valence in a novel way, the relationship between positive self-event connections and positive psychological functioning observed in the current studies cannot be directly compared to earlier research. This relationship, however, generally conform to prior work highlighting an association between psychological well-being and 'adaptive' (i.e. positive) forms of autobiographical reasoning and meaning-making within life story narratives more generally (King, et al., 2000; Lilgendahl \& McAdams, 2011; McAdams et al., 2001; Pals, 2006).

The relationship between positive self-event connections and positive psychological functioning was most salient in the context of narratives of difficult or negative events from the life story. By their nature, negative events can jeopardise individuals' well-being and cause psychological distress (e.g. Berntsen \& Rubin, 2006; Lyubomirsky, et al, 2006; Pals, 2006) have suggested that individuals are particularly vulnerable to experiences of psychological distress when negative life events become a central part of the life story. The findings presented in Study 1a (Chapter Four) suggest that making positive self-event connections in narratives of highly central, yet negative, life events may protect individuals from such psychological distress. That is, the relationship between psychological distress and endorsement of negative events as central to identity was reduced to non-significance for young adults who were most likely to make positive self-event connections in narratives of low points. Furthermore, young adults who made more positive self-event connections in narratives of central low points experienced better psychological well-being than young adults who made fewer positive self-event connections in narratives of highly central low points. These findings are consistent with a broader body of work which has shown that reflecting on positive outcomes or aspects of negative experiences moderates the relationship 
between the experience and negative psychological outcomes (Davis, et al, 1998; King, et al., 2000; Lilgendahl \& McAdams, 2011; McAdams, et al., 2001; Pals, 2006; Stein, et al., 1997).

Findings from Study 1a (Chapter Four) also suggest that for young adults who are less likely to make positive self-event connections in narratives of negative life events, engaging in the process of autobiographical reasoning may be associated with lower levels of psychological well-being. Pasupathi (2012) suggests that autobiographical reasoning is likely to be a dynamic process, such that connections drawn between past event and current self may change over time. As noted earlier, the cross-sectional nature of the data in Study 1a preclude firm conclusions being drawn. One possibility, however, is that for young adults who made fewer positive self-event connections in narratives of life story low points, more and more complex forms of autobiographical reasoning were associated with poorer psychological well-being because they were still in the process of searching for positive meaning (Steger, Oishi, \& Kashdan, 2009).

Relatedly, the findings of Study 2 (Chapter Five) showed that the number of positive self-event connections made in life story narratives (high, low and turning points combined) was unrelated to the degree to which young adults reported having meaning in their lives. However, for young adults who reported a relative of absence of meaning in their lives, making more positive self-event connections in life story narratives was associated with an increase in life satisfaction. The absence of a relationship between positive self-event connections and meaning is life was surprising given that autobiographical reasoning is conceptualised as a process by which individuals garner meaning from their experiences (McLean \& Thorne, 2003). One possible explanation for this finding may be, however, that for young adults who reported a relative of absence of meaning in their lives, making positive self-event connections is associated with the search for meaning in one's experiences. These possibilities highlight the clear need for future research examining changes in autobiographical reasoning in relation to important (particularly negative) life events and changes in reported meaning in life and psychological functioning over time.

The limited data presented in Study $1 \mathrm{~b}$ provide some support for the notion that patterns of autobiographical reasoning may not remain stable over time. In Study 1b, there was no relationship between the number of positive self-event connections found in young adults' life story narratives at two time points. That is, young adults who made more positive self-event connections at one time point were no more or less likely to make more positive self-event connections one year later. Furthermore, positive self-event connections did not predict changes in psychological functioning (as measured by symptoms of distress) over 
time ${ }^{11}$. As the events narrated at the two time points were not held constant in Study $1 b$, no conclusions can be made about changes in positive self-event connections in relation to single events over time. Unlike the findings for negative self-event connections (discussed later), however, these findings suggest that the tendency to make positive self-event connections in life story narratives may not be a stable individual difference. In the absence of evidence that making positive self-event connections is stable over time, it may be worth considering the possibility that this type of autobiographical reasoning is associated with coping efforts employed to regulate difficult emotional experiences on an 'as needed' basis. Theories of stress and coping (Lazarus \& Folkman, 1984) may be useful to consider in relation to this possibility.

Lazarus (1993a) defines coping as "cognitive and behavioural efforts to manage psychological stress" (p. 237). From a theoretical perspective, coping efforts are activated in contexts that individuals' appraise as harmful, threatening or challenging to their well-being (Lazarus \& Folkman, 1984; Lazarus, 1991; 1993b). Negative events that are perceived as central to one's life story and identity may conceivably fit into this category. Coping strategies can be differentiated according to whether they are 'problem-focused' or 'emotion focused' (Carver, Schier, \& Weintraub, 1989). Problem-focused coping' involves modifying the environment or one's behaviour in order to alter appraisals of the significance and emotional impact of the situation. Emotion focused coping in contrast can involve either altering the level of attention given to the situation (e.g. avoidance) or consciously altering the appraised meaning of the situation (Lazarus, 1995). Making positive self-event connections in relation to challenging events corresponds well to the latter component of emotion-focused coping. To illustrate this point further, consider the following example:

\begin{abstract}
"A low point which I have experienced in my life was when my parents moved from NZ to Australia. I was 20 when this event occurred. My parents decided that they wanted to live the rest of their lives in Australia instead of here. They had to sell the house in my home town and make a new life in Aus. At the time I was very upset of the thought of losing my parents and not having them in NZ. I was also sad to see the house I had grown up in being sold. I wanted my parents to stay but then I also wanted them to do the things in life that they wanted to and be happy. This shows that I am a caring and loving person who considers the feelings of others. I have developed over time because it made
\end{abstract}

11 Changes in psychological well-being over time were not measured in these preliminary data. 
me realise that my loved ones will always be there for me no matter how far away. It also made me think that I should consider the reasons as to why people make the choices in life they do before making any prejudgments"

This low point from the young adult's life story was rated as being highly central to their life story and identity (rating of 4.6 out of 5). Overall, this young adult demonstrated almost no symptoms of psychological distress and a high level of psychological well-being (an average sub-scale score of 63 out of 84). As evidenced in the written narrative of this experience, the young adult also made a number of positive self-event connections. These include links between the experience and a number of positive qualities they hold ("I am a caring and loving person who considers the feelings of others") and a number of positive insights and lessons they gained as a result of the experience ("I realise my family will always be there for me" and "I should always take others' perspectives into account before judging them"). As with the majority of autobiographical reasoning research, and the majority of studies in this thesis, the cross-sectional nature of the data prevents firm conclusions being made about causality. One possible explanation for these relationships, however, is that young adults' tendency to make positive self-event connections reflects a broader tendency to engage in emotion-focused coping strategies of which one component is to reappraise situations in a way that highlights positive characteristics of the self.

The relationship between cognitive reappraisal strategies and positive self-event connections is certainly consistent with this claim. As shown in Study 2, young adults who made more positive self-event connections in narratives of life story low points were more likely to engage in adaptive emotion regulation strategies (cognitive reappraisal) than were young adults who made fewer positive self-event connections in these narratives. Interestingly, the use of cognitive reappraisal strategies did not explain the relationship between positive self-event connections in narratives of difficult (low and turning point) experiences and psychological distress. This finding suggests that making positive self-event connections and cognitive reappraisal strategies are related but distinct constructs, with each being uniquely associated with psychological functioning.

Folkman, Lazarus, Gruen and DeLongis (1986) suggest that the use of positive reappraisal strategies is a reasonably stable coping trait. From a theoretical perspective, however, coping efforts change over time in response to situational and contextual demands (Lazarus, 1993b). Thus, the lack of stability in positive self-event connections found at two time points (Study 1b) may reflect participants' need to engage in coping rather than their 
general tendency to use positive self-event connections as a coping strategy per se. That is, young adults who demonstrate a stronger tendency to make positive self-event connections in general may construct life story narratives containing many positive self-event connections when actively attempting to regulate the impact of negative experiences and fewer when the impact of the negative experience is less severe.

An important area of future research would be to investigate these claims in more depth. An important starting point would be research examining links between the number of positive self-event connections observed in life story narratives and individuals' self-reported use of emotion-focused coping strategies. Measures such as the Ways of Coping Checklist (WCCL; Folkman and Lazarus, 1980) and some subscales of broader measures of coping strategies (e.g. the COPE Inventory; Carver, Scheier \& Weintraub, 1989) may be particularly helpful in assessing these relationships. If these basic relationships are established, future work could also examine the coping hypothesis via the use of an intervention-based study. A number of previous studies have had success in facilitating use of emotion-focused coping strategies among adults and children have found subsequent increases in positive adjustment (Kaluza, 2000; Pincus \& Friedman, 2004). Any resultant change in the number of positive self-event connections made in narratives of difficult events post-intervention (assuming increases in the use of emotion-focused coping strategies are observed) would provide support for the notion that these two processes are causally related. Furthermore, examining changes in psychological functioning pre- and post-intervention would illustrate whether these processes are in turn linked with mental health.

\section{The development of the life story and poor psychological functioning}

The studies in this thesis identified two main indicators of poorer psychological functioning. The first indicator was the number of negative self-event connections found in young adults' life story narratives. Young adults who made a greater number of negative self-event connections in narratives of life story events experienced more psychological distress (symptoms of depression, anxiety and stress), poorer psychological well-being and were less satisfied with their lives than were young adults who made fewer negative selfevent connections (Table 8.1). With the exception of McAdams et als'. (2001) 'contamination' sequences, autobiographical reasoning of a negative nature has not really been examined in the context of life story research. The relationship between negative selfevent connections and poor psychological functioning does fit, however, with the broad finding of McAdams et al. (2001), that narrating negative outcomes from life events through the life story reflects poor psychological functioning. 
The second indicator of poor psychological functioning highlighted in this thesis was young adults' perception that low points from the life story are a central part of their life story and identity. Interestingly, narratives of events that were considered central to the life story and identity contained more self-event connections than narratives of non-central events. Although not directly tested in the current thesis, these findings highlight a potentially negative cycle for young adults who are more likely to make negative self-event connections. In this proposed cycle (Figure 8.1), making more negative self-event connections in narratives of low points is associated with a stronger perception that these events are central to identity, which is associated with increased symptoms of psychological distress.

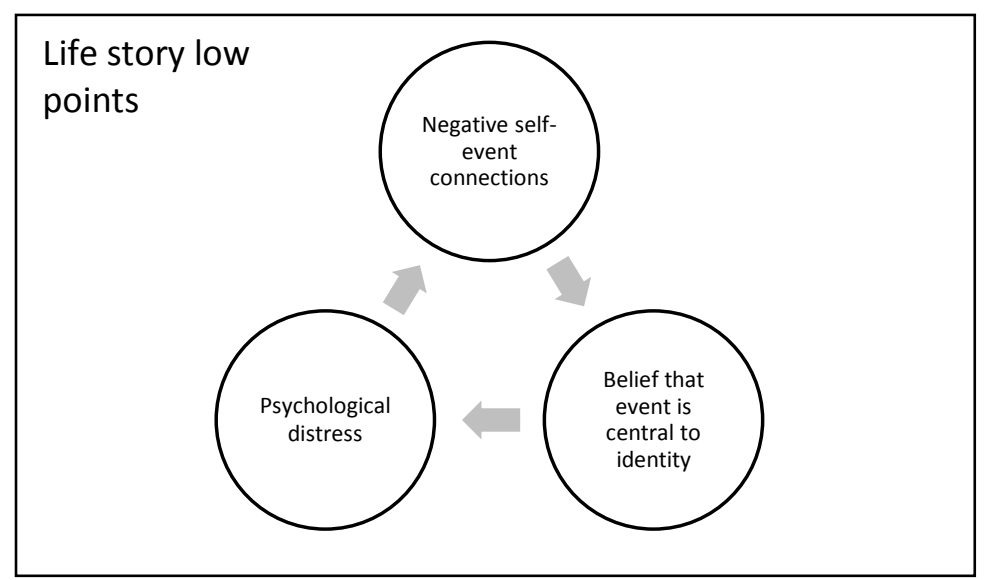

Figure 8.1. Hypothesised relationship between negative self-event connections, centrality of negative events and psychological distress

The findings of Study 1a showed that the process of autobiographical reasoning and life story development was associated with poor psychological functioning for some young adults, namely, those who demonstrated the strongest tendency to make negative self-event connections. For these young adults, engaging in more autobiographical reasoning (i.e. making more self-event connections) and more complex autobiographical reasoning (i.e. reporting more sophisticated insights into the self) was associated with increased psychological distress. This pattern was only observed in narratives of life story low points however, which highlights the important link between the way negative events are processed and psychological functioning (e.g. Lyubomirsky, Sousa, \& Dickerhoof, 2006; Pals, 2006).

In Appendix B, I present data showing that young adults who made more negative self-event connections in life story narratives were more likely to report that completing the 
life story task was a negative experience, and one that made them feel bad about themselves (see Table B1). These findings raise an ethical concern for research involving the construction of life story narratives. As life story researchers, we need to be aware that the tasks and measures we use may be unpleasant for some participants and may inadvertently cause them distress. Although the proportion of participants for whom completing the life story task was unpleasant was relatively small ${ }^{12}$, the balance between the need for scientific knowledge and the potential risk to participants must be considered. In the course of the current studies, I ensured that potential participants were aware that they would be asked to construct life story narratives in these studies and were given the option to withdraw their participation at any time. This was indicated in the participant consent information and communicated verbally to participants prior to participation. Participants who completed the studies were given a comprehensive verbal debriefing at the conclusion of the study. These debriefings were ideally completed one-on-one with participants and, where this was not possible, in small groups (i.e. two or three participants). Participants were also given a list of support services they could access and were encouraged to contact these services if they were experiencing distress. Ideally, future research should follow similar processes, ensuring adequate pre-participation information and post-participation debriefing information is provided, to reduce the potential negative impact of life story tasks on vulnerable participants.

The studies in this thesis broaden our understanding of the relationship between negative self-event connections and psychological distress by identifying a number of additional variables that relate to, and interact with, these constructs. The findings of Study 2 showed that young adults who made more negative self-event connections in narratives of difficult or challenging experiences (low and turning points) and adopted a negative explanatory style to explain negative events demonstrated more distress than young adults who demonstrated one or neither of these tendencies. Young adults who made more negative self-event connections in life story narratives overall, were less likely to engage in adaptive cognitive strategies (e.g. cognitive reappraisal) to regulate difficult emotions and tended to ruminate more about the cause of their distress. The level of rumination demonstrated by these young adults, in fact, accounted for the relationship between making negative self-event connections and symptoms of distress.

$1210 \%$ of young adults who participated in Study 2 reported that completing the life story task was a negative experience and $9 \%$ reported that the task made them feel bad about themselves. 
The significant mediation of the negative self-event connections to symptoms of distress by rumination raises the question of what are we measuring when we capture instances of autobiographical reasoning. One possibility is that current measures of autobiographical reasoning are, in part, measuring cognitive processes that explain the relationship between autobiographical reasoning and psychological functioning. From a theoretical perspective, autobiographical reasoning is distinguished from other forms of cognitive processing on the basis that it appears only in relation to the development of the life story. A small number of studies suggest that cognitive factors which influence the way individuals process their experiences can be readily detected in the way individuals describe events in their life story (Abela, Auerbach, Sarin and Lakdawalla, 2009; Adler, Kissel, \& McAdams, 2006). Abela et al., (2009) suggest that the influence cognitive factors such as core beliefs have on information processing can be observed through "spontaneous verbalisations on topics of personal significance" (p. 50). Furthermore, expressions of a negative explanatory style have been observed in narratives of life story events (Adler et al, 2006; Habermas et al, 2008). Although Abela et al., (2009) focused on the identification of core beliefs in narratives of life story events, a similar explanation may apply for a broader set of cognitive processes. In the case of rumination, one possibility is that negative selfevent connections are, in part, narrative products of the ruminative process. Individuals who ruminate spend more time thinking about their distress and difficulties (Nolen-Hoeksema, Wisco, \& Lyubomirsky, 2008). When this type of processing is engaged in relation to events that are included in the life story, it may increase the likelihood that negative self-event connections will be made and subsequently included in life story narratives. This notion raises the question of whether autobiographical reasoning reflects core cognitive processes that are not unique to the life story, but happen to be observed in life story narratives.

Future research using experimental manipulations of cognitive processes such as rumination may be used to highlight such effects. Rumination induction studies have had some success in increasing participants' use of maladaptive ruminative processes (Donaldson \& Lam, 2004; Hilt \& Pollak, 2012). Furthermore, rumination induction tasks have been shown to impact recall of autobiographical memories, highlighting the causal relationship between ruminative processing and autobiographical memory deficits associated with psychological distress (Watkins \& Teasdale, 2001). Future work that involves participants completing either a rumination induction or control (distraction) task before constructing life story narratives would allow differences in the number of negative self-event connections to be related back to this core cognitive process. In light of preliminary evidence that the 
tendency to make negative self-event connections remains stable over time (Study 1b), controlling for the number of negative self-event connections made at an earlier time point would give this design greater predictive power. Such future research should, of course, consider the ethical issue discussed earlier, namely the negative impact completing life story tasks may have on individuals who are prone to making negative self-event connections.

\section{Can Relational Frame Theory (RFT) explain the relationship between negative self- event connections and psychological distress?}

As highlighted in this discussion of findings, young adults who experienced symptoms of distress were more likely to construct life story narratives containing negative self-event connections. This is a new and interesting finding in light of prior work, which has largely focused on positive, rather than negative forms of autobiographical reasoning (Lilgendahl \& McAdams, 2011; Pals, 2006). In earlier Chapters, I introduced the theoretical notion that the life story comprises and individual's narrative identity (McAdams, 1988; 1993). If narrative identity is the life story, and poor psychological functioning in young adulthood is associated with the construction of a life story that contains negative autobiographical reasoning, then we may conclude that poor psychological functioning reflects also, the construction of narrative identity that highlights negative views or statements about the self. This theoretical assertion shares a number of conceptual similarities with a related theory of psychological functioning that has been gaining increasing prominence in the field of clinical psychology, Relational Frame Theory (RFT; Hayes, Barnes-Holmes, \& Roche, 2001). In the following section, I will outline Relational Frame Theory and consider whether and how this theory may explain the relationship between negative self-event connections and psychological distress.

The central tenet of Relational Frame Theory is that verbal humans possess the ability describe, explain, evaluate, interpret and rationalise their behaviour through language (Barnes-Holmes, Hayes, Barnes-Holmes, \& Roche, 2001). Through relations of stimulus equivalence, language develops the ability to represent actual and imagined objects. These equivalence relations between language and object are, in their most simple form, bidirectional. For example, when we see a cat or a picture of a cat, we might verbalise, visualise or hear the word "cat" in our mind. Likewise, when we hear the word "cat" or see the word "cat" written down, we imagine an actual cat. The concepts that come to be represented through language are not limited to concrete objects, but also include abstract concepts that may be used to describe a person such as "stupid", "funny" and "intelligent". In the same way that the word "cat" represents an actual cat, abstract terms such as the word 
"intelligent" represents a certain characteristic that a person can have (or not have). As children develop, they learn through their environment, that it is appropriate to label their own and others' behaviours in terms of these abstract concepts. For example, a behaviour such as performing well on a test may be labelled as evidence of "intelligence". When we too begin to relate to our own behaviour through language by labelling past and present behaviours in terms of abstract terms we develop a conceptualised self - a network of selfdescriptions and self-evaluations that we use to explain our behaviour (Barnes-Holmes, Hayes, \& Dymond, 2001).

Unlike concrete objects, the truth (or true existence of) abstract concepts that comprise the conceptualised self are difficult to verify (Barnes-Holmes, Hayes, \&Dymond, 2001). It is much harder to verify that someone is "stupid", given the obvious difficulties associated with defining and operationalising, let alone measuring, this concept, than it is to verify that cats actually exist. Our minds do little to address differences in the veridicality of these equivalence relations, however, and abstract self-descriptors can exert the same degree of control over our behaviour as can concrete terms. For example, upon hearing someone say "look, a cat!", we may turn our heads and perhaps even walk up to pat the cat. Verbalising abstract self-descriptors, either internally or verbally to others can act in a similar way. For example, the student who thinks to themselves "I failed that test because I'm stupid" will believe that they are in fact, a 'stupid' person. As a result, this student may feel distressed and hopeless and decide not to sit the end-of-year exam because they expect to fail.

From a theoretical perspective, the conceptualised self can be problematic for psychological functioning when verbal descriptions of the self are largely negative in nature, rigid and pervasive, and gain a greater control over one's behaviour than environmental contingencies (Fletcher \& Hayes, 2005; Kanter, Busch, Weeks, \& Landes, 2008). Negative self-descriptions can become pervasive and rigid as relational networks of negative internal states, thoughts, memories, behaviours and environmental cues increase in strength via repeated pairings. Again, consider the example of the student who thinks "I'm stupid" in relation to a failed test. This student is likely to be exposed to future situations which have similar characteristics to this earlier situation, for example, sitting their driver's license test. In this second situation, some of the environmental and internal cues associated with sitting a driver licence test (e.g. nervousness, anticipation, the presence of an examiner) may be similar to those present in the first testing situation. These internal and external cues may then, via relational frames, activate the memory for the prior situation (finding out that they had failed), the thoughts and emotions that were experienced in that situation (sadness, 
despair, "I must be stupid") and the consequences of these internal experiences (hopelessness, deciding not to pursue a university degree). Activation of this relational frame is not only likely to cause the individual a significant amount of distress, but also via repetition and the new associations to further environmental and internal cues, increases the salience and pervasiveness of the negative self-description (Wilson, Hayes, Gregg, \& Zettle, 2001).

There is a large degree of overlap between the theoretical definitions of the 'conceptualised self' and 'narrative identity'. The first similarity is in the formation of the conceptualised self and narrative identity through linking descriptive and evaluative information about the self to one's experiences. From a theoretical perspective, the life story and narrative identity is developed through autobiographical reasoning. Autobiographical reasoning occurs when an individual describes an aspect of themselves such as a trait, characteristic or quality they possess, and links this self-description to a memory for a past experience (Habermas \& Bluck, 2000; McLean et al., 2007). The conceptualised self is formed by the activation of descriptive and evaluative self-statements that are linked to external (e.g. environmental) and internal cues (e.g. thoughts, emotions, physical sensations, memories and mental images) through relational frames.

The second similarity is that both models propose that links between self and experience are constructed through language, or narrative. Life story models of identity propose that links between past event and current self (evidence of autobiographical reasoning) are constructed through narrative, whether it be a narrative that is communicated to others or internalised and retained as private to the self (McAdams, 2001). Relational Frame Theory proposes that the conceptualised self develops through language, which is the tool that we, as humans, have to represent abstract constructs such as traits and personal characteristics. These models both suggest that language is a symbolic tool that we use to represent and understand our world. The symbolic nature of language can, however, be overlooked and, when applied to ourselves, has the power to influence and determine the beliefs we hold about ourselves.

Where Relational Frame Theory extends beyond the theoretical claims of the life story model of identity, is in its ability to account or individual levels of psychological functioning. RFT posits that the conceptualised self can maintain symptoms of distress because negative self-statements are a) accepted by the individual as truly representing an aspect of the self, b) are triggered by a range of internal and external cues and c) as a result of their perceived truth and pervasiveness, prevent individuals from experiencing environmental contingencies that disconfirm the self-belief. That is, the student who believes they are 
'stupid' may demonstrate a bias towards environmental events that confirm this belief (or which appear to confirm this belief). For example, forgetting to turn the oven off may be seen as evidence of this stupidity, rather than a consequence of being distracted by an unrelated event (e.g. having an extremely busy evening or someone knocking on the door).

A question for research examining the links between life story development and psychological functioning, is whether this explanation adequately accounts for the relationships observed between negative self-event connections found in life story narratives and poor psychological functioning. Psychological therapies that are based on Relational Frame Theory, namely Acceptance and Commitment Therapy (ACT; Hayes, Strosahl, \& Wilson, 1999) may provide one avenue for examining this claim. ACT encourages the development and use of six core processes. One of these processes, termed 'self as context', is directly aimed at reducing the power and control of negative self-descriptors on individuals' behaviour and emotions. The notion of self as context refers to the experience of being aware of, and engaging with, the environment in the present moment. Clients are encouraged to attend to their environment without allowing evaluative thoughts about themselves, their experiences, or others to enter their mind. In doing so, negative thoughts about the self become less 'believable', and come to be accepted as merely fleeting internal events with very little truth value. Preliminary evidence suggests that developing these skills can reduce symptomatology among individuals with PTSD, and who believe their traumatic event is central to their identity (Boals, 2012). Further research aimed at developing these skills among individuals who have a tendency to make negative self-event connections in the life story and tracking changes in psychological functioning before and after these skills are developed will address the validity of the claims made here.

\section{Future directions}

The findings presented in this thesis could be usefully extended by considering gender and age differences in the relationship between autobiographical reasoning and psychological functioning. Young adults were chosen for examination in the current studies as the development of the life story, and hence identity, is a theoretically significant achievement during this developmental period (Habermas \& Bluck, 2000; McAdams, 1988; 1993). Highlighting this developmental difference, Pasupathi and Mansour (2006) found that the likelihood of constructing life story narratives containing autobiographical reasoning increased between adolescence and young adulthood and decreased in older adulthood. Thus while the presence of autobiographical reasoning in life story narratives may be associated with positive psychological functioning in young adulthood, it may not show a similar 
association to psychological functioning in older adulthood. The findings of Study 1a show, however, that the valence of autobiographical reasoning is a stronger predictor of psychological functioning than the presence of autobiographical reasoning per se. Given the theoretical importance of autobiographical reasoning in young adulthood, autobiographical reasoning valence may be a stronger predictor of psychological functioning in young adulthood than it is in older adulthood.

Gender differences in the relationship between autobiographical reasoning were not able to be examined in the current studies due to the relatively small proportion of males who participated. As mentioned in various Chapters, there are some qualitative differences in the way males and females construct stories about past events. Namely, females typically construct longer narratives, and include more emotional and evaluative terms in their narratives than do males (Fivush\& Buckner, 2003). These gender differences may illustrate normative differences in autobiographical remembering that are learnt from an early age. As outlined in earlier Chapters, mothers tend to be more elaborative when recalling past events with daughters than with sons, particularly in relation to emotional experiences (Reese, Hade, \& Fivush, 1996). No differences in the amount of autobiographical reasoning found in life story narratives have been found between older adolescent males and females, however (McLean \& Thorne, 2003). Thus although we might not expect to see gender differences in the amount of autobiographical reasoning found in life story narratives of young adults, gender differences may exist in the purported use of autobiographical reasoning. Females may be more likely to use autobiographical reasoning to process difficult emotional experiences than males. We may therefore expect that the relationship between autobiographical reasoning valence and psychological functioning may depend on the extent to which these emotional experiences are resolved, or in the process of being resolved (see Pasupathi, 2012).

In the four studies reported in this thesis, life story narratives constructed by young adults were examined for autobiographical reasoning valence, the extent to which past events were linked to positive and negative characteristics of the self. The findings of these studies make a number of important contributions to the growing body of research examining links between autobiographical reasoning and psychological functioning. First, psychological functioning in young adulthood was associated with the extent to which individuals' reason about the self in positive and negative ways in the context of the life story. Reasoning about the self in positive and negative ways was also shown to be associated with a range of additional factors that had not yet been considered in prior work. A more complete 
understanding of the relationship between autobiographical reasoning valence and psychological functioning may be gained by considering how these additional factors (cognitive response styles), meaning in life, and autobiographical memory phenomenology may relate to the process of autobiographical reasoning. The implications of these findings may also be usefully considered in the context of models of coping and Relational Frame Theory (RFT; Hayes, Barnes-Holmes, \& Roche, 2001). 


\section{Appendix A}

\section{Themes within young adults' life story narratives}

The purpose of this Appendix is to present key themes in the life story narratives written by young adults who participated in the studies comprising this thesis. As discussed in Chapter One, the events included in the life story are guided by cultural norms regarding the events which are likely to occur in a 'prototypical life'. Empirical work supports the existence of the cultural life script and in Western cultures, has converged on 35 key life events that are likely to be experienced over the course of the lifetime (Rubin, Berntsen \& Hutson, 2008).

Previous research examining themes in the types of events described in life story narratives have tended to use broader categories than the list of events outlined in the cultural life script. For example, events such as attending university, beginning school and attending high school that appear in the cultural life script have been classified according to a broader category 'educational events' (Tavernier \& Willoughby, 2012). This broader classification of life story events allows a greater number of events to be grouped into meaningful categories. For example, individuals may include the day they passed their final high school exams as a high point in the life story, which is related to the general life script event of 'attending high school' but is not fully accounted for by this life script event.

\section{Coding narrative themes}

Themes within narratives of high points, low points and turning points were identified using the event type categories first reported by McLean and Pratt (2006) and adapted by Tavernier and Willoughby (2012). This coding scheme was originally created, and in prior work has only been used, to categorise narratives of turning point events. Upon inspection of the narratives collected in the current research, it became clear that these pre-defined categories would also account for the majority of high point and low point events (see Table A1). The original coding scheme consists of five event-type categories: relationship events (interpersonal experiences, changes or conflicts with friends, family and intimate partners), travel events (experiences involving travel to another country and experiencing a new culture), moving events (permanent relocation to a different town, city or country), mortality/life-threatening events (experiences involving bereavement, major illnesses or accidents for the participant or close other), educational events (events relation to academics, career choices or university plans). 
While the five main event categories accounted for the majority of narratives collected in the current studies, a significant proportion of narratives were coded as 'other'. These narratives were then reviewed for additional themes. A number of additional themes emerged during this review and were subsequently added to the coding scheme. These additional categories included: sporting events (events involving participation and/or success in a sporting activity), holiday events (vacations and recreational trips; high points only), other achievements (successes in domains other than educational and sporting; high points only), mental health-related events (experiences involving mental illness or psychological distress for participants or close others; low and turning points only) and identity-related events (events that challenged or highlighted issues of sexuality, race or other identity domains; turning points only). The original coding scheme also included a category for responses that were vague or responses that did not describe a particular experience (no event). Over the course of the current studies, 460 high point narratives, 461 low point narratives and 266 turning point narratives were collected. The following section provides a summary of key themes and excerpts from participants' life story narratives.

\section{Relationship events}

Overall, the most common theme in young adults' life story narratives was relationships. Approximately one-fifth of high point narratives (21\%), one-quarter of turning point narratives $(24 \%)$ and one-third (31\%) of turning point narratives were based on events involving relationships with others. Prior work examining themes in turning point narratives written by adolescents reported a similar proportion of relationship-related events (23\%). Relationships are highly salient during the adolescent period as young people gravitate away from parent-child relationships and learn to navigate complex social relationships with peers (Collins, 1997). McLean and Thorne (2003) found that adolescents' views, beliefs and thoughts about changes in, and differences between, their relationships with parents and peers were readily observed in narratives of self-defining memories. As would be expected, the types of relationship events described in narratives of high, low and turning points differed. Some of the major themes in each of these narrative types will be presented.

\section{Relationship events as high points}

Many of the relationship events discussed as high points from the life story were based on experiences of gaining a romantic partner, or described occasions where participants had spent time with a romantic partner: 
"There was this girl I really liked from a different school. We were friends for a while but I had a big crush on her ... After a long time, I asked her out and she said yes. She was my first ever girlfriend and I was ecstatic ... This gave me a good self-esteem boost. Having this girlfriend has changed me because it has taught me how to care for someone and being able to listen"

"At age 17 I met my current boyfriend ... A few months after my 18th birthday he took me to meet his parents. We were staying all weekend and on the Saturday night we went to walk along the beach near the family home and that was where he told me he loved me for the first time. I had never felt so happy in my life ... I was so happy it brought tears to my eyes ... all the emotions associated with this moment make me happy and content with my life"

Other relationship events that were a high point from the life story were based around special times spent with friends and family:

"The high point in my life was when my friend flew up from Dunedin to surprise me for my 18th birthday ... I was the happiest and most excited I had felt in a long time. I couldn't believe what an amazing friend she was for giving me the best surprise I could have asked for. She made it an amazing 18th birthday ... This was an important event in my life as it made me feel extremely special. It showed me how much people, especially my friend, cared about me. It shows me that people value my friendship just as much as I value theirs"

"I was 17 when my family and I went on a trip to Malaysia to see family ... I remember feeling especially happy one day when we celebrated my grandmas 77th birthday ... It was such a joyous occasion being around family we see only every 5 years or so ... I think this event was important in my life because it made me see that nothing is depressing or sad forever, you will always get moments in life that make you happy"

"Christmas when I was 17 years old ... This particular Christmas was a high point for me because my parents [gave me] a card and some money ... Inside the card was a long message from mum and dad telling me how proud they were of how hard I had worked this year, of the young women that I had become and of the way that I had handled everything over the year. I still have this card. My parent's approval in me was one of the best gifts that they had ever given me. This event is 
so special to me because it reflects what I find important in life- my family and doing right by them"

\section{Relationship events as low points}

Unlike the types of relationship events reported as high points from the life story, which were positive in nature, relationship events reported as low points were negative in nature. The large majority of relationship events discussed in narratives of life story low points described conflicts or difficulties that arose in the context of relationships with friends, family and significant others.

"When I was 16 I went through a rough time in a relationship, involving someone who I really cared for. We had a really good friendship all throughout school and then in 6th form got together ... Things turned very pear-shaped and we started having problems ... most of it was due to mistrust, misunderstandings, jealousy, and lying ... when things turned bad I remember this being one of the lowest points in my life ... I was sad, depressed, upset all the time, felt vulnerable and tired and like I was constantly going to break down in tears ... I have learnt to have more trust in people, but also not to be fooled by believing rumours from dodgy sources and to talk about things before they boil up and become a major issue"

Relationship break-ups were also a common theme in low point narratives of relationship events. These included both the separation of participants' from romantic partners and the separation or divorce of their parents. Parental separation often led to further changes in family relationships and the amount of contact participants had with other family members.

"When I was 17 years old I had been with my boyfriend for just over 3 years when he rung me up one morning and told me that he had slept with another girl at a party in the weekend. I was so distraught. It was such a shock. Although he sounded very apologetic I knew I would never be able to trust him the same afterwards. We broke up because of this ... He was and always will be a huge part of my life story. He taught me many things about myself, life and about love that I will never forget ... I feel stronger after this and now can move on knowing that if I can through this then I can get through anything" 
"A low point in my life is when my parents separated 2 years ago. This hurt and shocked me as I didn't see it coming. It separated me and my sister from my brother as we lived with different parents ... As my sister and I didn't have a mother figure anymore, I became that figure to protect my sister from being lost. I remember thinking that it was hard, and feeling sad but wanting to stay strong for my sister. This event has changed who I am today as I learnt a lot about people and emotions. I learnt a lot about responsibility and priorities also ... I realised that there isn't anything you can do when you are in a situation like that and just to do your best"

\section{Relationship events as turning points}

Relationship experiences that were described in narratives of turning points often involved a change in participants' relationships with friends, romantic partners and family members. Many of these changes to relationships were the result of negative experiences.

"In seventh form (I was17 years old) the girl I considered my best friend randomly decided she would stop talking to me and started acting like I was invisible. It was totally unprovoked and came out of nowhere. When I eventually confronted her about it, she said the other girls in our group had told her that we spent too much time together. I was really [upset] by this ... This has affected me in the way I am with new friends, I find it harder to fully trust people"

"When I was 18 I entered into a relationship with someone for the first time ... I was with this person for nearly two years ... The person involved suffered from depression, and dealt with this though heavy use of drugs, an aspect of their personality that I was unaware of until a few months into the relationship ... That person was my priority over my friendships and my university life, which as a result lead to bad grades, barriers arising between friendships, and a decreased well-being as I became severely anxious ... However, I have now managed to move on from this person, a process which took a long time to do ... It was definitely an experience that I was unprepared for, however, I reflect upon it now as a turning point that taught me resilience, and maturity"

“Growing up I didn't have a dad so I lived alone with my mother and twin brother. We were not a wealthy family by any means as my mother was a stay-at-home mum looking after me and my brother ... At the age of 6 we moved to from 
Auckland to Wellington and my mum meet a man who would soon become my step father. We almost immediately moved in and they got married after about a year. He then adopted me and my brother and effectively treated us like his real sons. All our life we wanted that father figure and I believe that having a dad changed my life considerably"

\section{Educational events}

Overall, the second most common theme in life story narratives provided by young adults in the current studies were educational events. Educational events were significantly more likely to be reported as high points from the life story than low or turning points. That is, educational events comprised one-quarter (24\%) of events discussed in high point narratives, $8 \%$ of low point narratives and $14 \%$ of turning point narratives. The proportion of education-related events was higher in the current studies relative to the proportion of turning point events reported in earlier work (Tavernier \& Willoughby, 2012). The prevalence of education-related event in high point narratives most likely reflects a key characteristic of the sample who participated in this research. Participants in the study by Tavernier and Willoughby (2012) were secondary school-aged adolescents. Participants in the current study were undergraduate university students. Given these participants developmental stage, their age, their decision to attend university, and the high standards for attaining university entrance, it is reasonable to expect that educational events may play a salient role in the life story of these participants.

\section{Educational events as high points}

Participants' experience of finishing secondary school was a common educational event described in young adults' high point narratives:

\footnotetext{
"A high point in my life was walking across the stage with my mother in hand at my graduation from high school. I was feeling very proud and ecstatic at the fact that I had passed all NCEA levels and had planned to go Victoria University. This event is such a high point for me because on that day I had been rewarded all the hours that I sacrificed not to go out or to parties but to stay home and study. This event is important because it showed me that yes, if you do work hard and stay focused you will succeed in whatever you do"
}

In addition to finishing high school, many educational events discussed as high points from the life story involved gaining entrance into, and starting, university: 
"A high point in my life was getting in to university ... It was exciting as I felt like I was starting something new and there were many opportunities for me ... This event was important as it says that I am a hard working person and know when to put the effort in. It is also important as over these next few years I am going to change and mature as a person. What I do over the course of my degree will also play a huge role in what career path I take in my life"

Academic achievements such as attaining good grades, receiving academic awards and excelling at other academic pursuits were also common education-related high points:

"When I received my first year law results which were 'A' grades ... When I looked at my results, I was so nervous that my heart was pounding and I felt I could not breathe ... when I saw that I had received A grades I felt the sweetest sense of disbelief and joy ... This is an important event in my life story because when I saw my results I decided I wanted to continue [studying] law"

"When I was 13 I received the highest grades for my year. I was at prize-giving and was called up to receive the award for the top of year 9. The principal shook my hand and the audience clapped. I was very happy and pleased with my achievement. This event shows how capable of success I am, it shows how much potential I have"

\section{Educational events as low points}

Although relatively few low point narratives (8\%) described an educational event, where young adults did describe an educational event as a low point, these events often described instances where participants were not successful in academic pursuits or struggled to meet the demands of their academic courses:

"A low point in life would most likely have to be when I was at [secondary] school around year 10 - 11, so I was 16 years old. The event was low because I was not coping very well at school and especially in my studies, I felt I had to compete with my older brother for high grades and I couldn't do that ... I gave up trying and just let my grades slip ... I feel it is important event in my life as I have learnt to deal with situations on my own and become more independent. It also has taught me (due to passing the year badly) that I should work hard and get good results for myself not for my family or in comparison to my brother" 


\section{Educational events as turning points}

Many educational events described in narratives of turning points involved changes or transitions, for example, from primary to secondary school or from secondary school to university:

"A turning point would be when I started high school. Over a period of about 2 years I became much more confident and much less shy, which I think was important for me! I realised that I can talk to people and it's not so scary. I also made more friends, joined a jitsu club, and all of this made me content with life throughout high school. This event shows how over time I have developed into a much more mature, confident person who can take charge of their life"

"For me the transition between no longer being a high school 'child' and the transition of being a university 'adult' was quite significant ... For me this was a hard time of life as [I had] to make some big decisions that will impact [my] future considerably. I remember the decision over whether or not to start a degree was an incredibly daunting prospect for me ... I think this transition and feeling this way about it is incredibly normal for young people in the same situation and it's just a part of becoming an adult"

Although not directly related to academics, many educational events described in turning point narratives were based on being awarded leadership positions with the school participants were attending:

"[At] 12 Years of Age, [I] was given the responsibility of being a school ambassador ... This is a turning point in my life as I think it taught me to be responsible and to be a role model to the people around me ... Looking back now, this event made me grow as a person in becoming a good role model to my peers and to be responsible for my actions and for those around me" 


\section{Mortality / life threatening events}

One in three (32\%) narratives of low points from the life story, and one in ten $(10 \%)$ narratives of turning points from the life story were based on events concerning mortality (particularly loss of a loved one), major illnesses and life threatening events or accidents involving themselves or others. As would be expected, no narratives of life story high points described mortality or life-threatening events. Tavernier and Willoughby (2012) found that mortality / life -threatening events were the most common theme of turning point narratives written by adolescents $(27 \%)$.

\section{Mortality events as low points}

Many low point narratives written about events involving mortality described the death of a grandparent to whom the participant had a close relationship. This is not surprising as, given the age of participants; the death of a grandparent is likely to be a more common occurrence than the death of friends, siblings and parents:

"I was about fifteen when my nana passed away, she had always been a big part of my life and my sisters. It was really hard on both of us as my sister had only just moved away to university and we'd always been there for each other. It was at this time that I felt most alone and that I had no-one to turn to. I had seen my nana as a survivor because she'd fought breast cancer twice already but the last time she didn't have the strength. I refused to tell my friends what had happened and alienated myself because I felt that as long as I wasn't close to anyone no-one could hurt me ... After this I don't think I have ever been the same, I don't let myself get too close to people in the fear of getting hurt"

Accidents or life threatening illnesses experienced by the participant, other family members or their friends also featured in a number of low point narratives:

"When I was 17 my dad [was] diagnosed with cancer. He went through a lot of tests and operations within the last 8 months. At the time I felt frightened and nervous as to how the results of each test and surgery and overall dad's health were to be ... This event is important in my life story as I dealt with a variety of emotions not just from myself but also experienced the emotions indirectly through my family and friends response to the event. It has made me appreciate every moment with my dad and view life through a more positive set of goggles than what I use to view life as" 
"In 2007 when I was about 17 I was involved in a car crash due to a drunk driver losing control over his car ... During this situation I went into shock mood and my memory of it all happening felt really quick ... This is an important event because it taught me about how careful you need to be on the roads and that we were all lucky nothing fatal happened to anyone"

\section{Mortality events as turning points}

Similar to mortality events described in low point narratives, mortality events described in turning point narratives tended to be based on loss of a loved one, major illnesses and life threatening events or accidents:

“An important moment in my life was when my mother's best friend died of cancer. She was like a second mother to me and her daughter was my best friend ... She had been diagnosed with breast cancer about a year and a half before she died and this was a huge turning point in my life. I had never experienced any kind of grief before this point ... It made me realise what the important things in life really were. Doing badly in a math's test or exam isn't the end of the world and this moment in my life made me realise this, and put things into perspective"

"Last year, when I was 16, I got very sick with glandular fever and its associated illnesses. I was off school for 6 months ... I felt quite alone at the time as I had to be shuffled around different family members so they could look after me. This is an important even in my life story as it forced me to re-evaluate my goals for the next year. University looked out of the picture. But I somehow managed to gain university entrance. Getting sick made me realise that situations can change quickly and this has changed me as a person and no I take nothing for granted"

"I was 19 years old when I went through a traumatic ordeal that changed my life. In this event I woke up to a violent shaking of my house and it took me a few seconds to realise that I was experiencing a major earthquake ... This was an important event in my life story because it changed me dramatically. I now have a fear of certain buildings; I can't attend movies at the theatre because loud noises make me feel anxious. I am consciously aware of exits and ways out of buildings in case I need to evacuate. Before the earthquake I was very relaxed and lived freely, I didn't worry about getting hurt or about any life changing events because I didn't think they would ever happen to me. This event has definitely shaped who 
I am now, it has made me more empathetic and understanding, but has also made me fear things within the world which I would have previously never thought about or feared"

\section{Moving events}

One-quarter (24\%) of turning point narratives written by young adults in the current studies described an event which involved moving or relocating to a different city or country. The proportion of moving events reported as turning points in the current studies was much higher than that reported in prior work with adolescents (Tavernier \& Willoughby, 2012). Again, this likely reflects the age and developmental stage of participants in the current study. Many of the young adults who participated in the current studies had recently moved to Wellington from another part of New Zealand in order to study at Victoria University. Thus not only was the experience of starting university a salient experience (see earlier discussion 'Educational events as high points), for many of these young adults a major transition involving relocation to Wellington had occurred in the recent past.

\section{Moving events as turning points}

Reflecting this characteristic and developmental stage of participants in the current studies, many turning point narratives describing moving events were based on the shift from participants' childhood home to Wellington in order to begin their university studies.

"One turning point in my life was when I first went to university. I was 18 years old, and as I had to move to Wellington, I went into a hostel. This marked the first time I moved out of home, and was also the first time where I was put into an environment where I could not fall back on family and my long term friends. I found it very hard at first, but after a while I got used to it and came to enjoy having new freedoms and responsibilities. I felt this was important as it shows that I could adapt to new situations, as well as being the first milestone in becoming independent and self-motivated"

Also, potentially a reflection of New Zealand's ethnic diversity, moving events described in turning point narratives commonly referred to participants' experience of emigrating to New Zealand from another country as a child. These events were often perceived negatively by participants at the time of the move, however many were able to see positive outcomes from the move on reflection. 
"A major changing point of my life would be when I left my hometown of Singapore at the age of 5 and came and lived in NZ with my family. I had grown up in this supportive and close group of friends and family in a place which I loved, and suddenly all this was about to be taken away from me and I was going to have to move to a new place where I knew no one ... I feel that although my life would have been just as good if I remained in Asia, I am glad of the fact that I had a challenging youth even which caused me to develop as a person from a young age, and has since strengthened me as a person"

"I was 11 years old when I moved to NZ from England, with my family. This was a huge turning point in my life because I was moved to the other side of the world. I thought it wouldn't be that different, but there is a huge variance in culture ... At school we learnt about the Treaty of Waitangi and through my classes' eyes, it was all my fault. Even my teacher directed her hate over the events that had happened towards me. [I was] feeling powerless at school, feeling powerless in the decision to move and feeling powerless over my emotions, I have grown a great need to be in control over every situation"

\section{Moving events as high and low points}

Events involving relocation to another city or country were less commonly described in narratives of high points (7\%) and narratives of low points (4\%). As with moving events described in turning point narratives, moving events described in high point narratives were tended to centre on a permanent relocation to New Zealand from another country or moving to Wellington where participants currently resided.

"A high point in my life would be moving to New Zealand. I was 15 years old and at first it seemed like it would be horrible, moving to an entirely different country (I was moving from America) and not knowing a single person here other than my dad and sister ... Eventually I just settled in though and I have met so many amazing new people from this. It is important in my life story because it has definitely changed me. I was very shy at school and having, being forced, to meet new people and introduce myself made me come out of my shell”

"A high point for me would be when I moved away from my small town to Wellington, which seemed like a massive city at first. It was so exciting ... The 
move was such a positive experience for me and I feel as though I would not be the person I am today if I had of spend this year stuck in small town"

In contrast, low point narratives that described experiences of moving tended to occur when participants were young and had little control over the move. These experiences of moving at a young age were perceived in a negative way:

"At the age of 15, my parents moved me over to New Zealand. Because of my age I was still under guardianship rules and so I had no choice in the matter, and had to move with my parents ... I hated my parents for moving me, even though they tried to cheer me up and told me they understood what they were putting me through and that they were sorry. The emigration was a very important event in my life. It changed who I am as a person because it taught me that things don't always go the way you want them too, and often you have to make the best of things ... Since having moved to New Zealand, I have been to the UK [United Kingdom] twice. I still see it as home, and miss it heaps"

\section{Sporting events}

Just over one in ten (13\%) high point narratives described a sporting event. This category was not included in the original list of event types by Tavernier and Willoughby (2012) however was identified as a common theme in the narratives provided by young adults who participated in the current studies. The prevalence of sporting events in narratives of the young adults who participated in these studies may reflect the value on, and popularity of, sporting activities in New Zealand. In New Zealand it is common for young people to be involved in sporting activities during their childhood. The high level of engagement in sporting activities naturally exposes young people to successes or otherwise in this domain.

\section{Sporting events as high points}

Consistent with this claim, sporting experiences described in high points mostly involved achievements such as winning important games or tournaments, being selected for prominent sports teams and representing one's region or country in sporting events.

"A high point in my life would have to [be] when I was around 15 and I won a gold medal in a National New Zealand Karate tournament ... I was ecstatic that I had won and standing up on the podium I felt a rush of happiness and was filled with endorphins ... I think this is an important event in my life story because It 
shows that you can do anything if you try hard enough and put your mind to it. This event says that I am a determined individual with a competitive spirit"

"A high point I experienced in my life was being selected for the New Zealand Under 20s football team ... After going through a week long training/selection camp, I was informed of my selection in the team a week later ... The first thing I felt after hearing the good news was a bit of shock and I remember thinking to myself did he really just say I made the team? But that feeling was very shortlived and was soon replaced by an overwhelming feeling of pride, joy and excitement because I would be representing my country in the sport that I love"

"I represented New Zealand in the World Mountain Running Championships after winning the New Zealand Junior Championships ... I was the only one from New Zealand and travelled with my mum to Albania which is a very, very different country to New Zealand. I was a little bit anxious before the start as I had never competed at such a high level before in 40 degree heat against people I didn't know. I think this is an important event in my life because it taught me how to cope in different environments - I had to live in a different culture before the race, eat different food, adjust to heat etc."

\section{Sporting events and low and turning points}

Sporting events were infrequently described in narratives of low points (2\%). Similar to the description of sporting events in high point narratives, sporting events described in narratives of turning points (7\%) predominantly centered on sporting achievements that had a lasting impact on the participant:

"I was 16 when a major turning point occurred in my life. The major event was winning a medal at the New Zealand secondary school rowing championships ... I don't think I have even been as nervous as I was In the starting blocks of that race ... However whilst in the race I found myself incredibly calm and collected. A voice kept telling me you can do this, and think of all the years of hard work that have led to this moment. Crossing the finish line in 3rd was one of the greatest moments of my life. I felt a huge amount of elation. This event was so important to me, as it showed me that with perseverance and dedication comes success and that I should keep striving in life despite the pitfalls that may sometimes lie along the way. 


\section{Travel events}

Approximately one in ten (13\%) high point narratives and 6\% of turning point narratives were based on an experience involving overseas travel. This is slightly higher than the proportion of turning points describing travel events by Tavernier and Willoughby (2012). Again, this difference may reflect the older age group of participants in the current study and cultural norms present in the New Zealand context. It is common for young people in New Zealand to travel overseas after finishing their secondary school education. It was noted however, that the majority of travel related experiences narrated in high and turning points occurred when young adults were still at school. These experiences often described the learning and personal development that accompanied exposure to another culture. In many cases, these experiences changed the participants' view of the world and their culture and sparked in them an interest in further travelling:

"When I was 15 , I went on a school trip to Japan. It was my first time overseas, so I was incredibly excited to be leaving New Zealand and experiencing a new culture. I travelled with some classmates, two teachers, my brother, and some very good friends ... I think it is an important event in my life story because it changed my entire perspective on life. It opened my eyes to different ways of life, and showed me the diversity that exists around the world. It encouraged me to think about things with a more open mind. It helped me to evaluate my beliefs and develop a new-found appreciation for what I have. It definitely stemmed an interest in learning about even more cultures, influencing my decision to major in Anthropology, because it was my first first-hand experience of another society"

"When I was 14, me and my family went on a holiday to Rarotonga. I really enjoyed the holiday as it was my second time out of the country and because I was old enough it opened my eyes to realising that travel is an important thing I want to do in life. While I was over there I loved the differences geographically, culturally, and in temperature from New Zealand to name a few. From this experience and others I now know that travel is something I really want to do. It made me click that there is a lot to see around the world and since then the prospect of travel has always been in my mind"

\section{Gender differences in narrative themes}


Chi-square analysis showed that female participants were significantly more likely to write about experiences involving relationships in the context of life story turning points than were male participants. Male participants on the other hand were more likely than female participants to narrate educational events as a turning point in the life story. There were no other significant gender differences in the themes of turning point narratives. Males were significantly more likely to narrate educational events as a low point in their life story than were females. There were no other significant gender differences in the themes of low point narratives. There were no significant gender differences in the themes of high point narratives. 
Table A1

Themes in narratives of low points, high points and turning points from the life stories of young adults

\begin{tabular}{|c|c|c|c|c|c|c|}
\hline Category & Example & $\begin{array}{c}\text { Total } \\
(\mathrm{n}=1,187)\end{array}$ & $\begin{array}{l}\text { High points } \\
(\mathrm{n}=460)\end{array}$ & $\begin{array}{l}\text { Low points } \\
\quad(\mathrm{n}=461)\end{array}$ & $\begin{array}{l}\text { Turning } \\
\text { points } \\
(\mathrm{n}=266)\end{array}$ & $\begin{array}{c}\text { Tavernier \& } \\
\text { Willoughby } \\
\text { (2012) }\end{array}$ \\
\hline & & $\%$ & $\%$ & $\%$ & $\%$ & $\%$ \\
\hline Relationship events & $\begin{array}{l}\text { "I was with my girlfriend and she told me she loves me - that was the happiest } \\
\text { I've been" }\end{array}$ & 26 & 21 & 32 & 24 & 23 \\
\hline Travel events & $\begin{array}{l}\text { "when I was } 18 \text {, I went over to the UK for a gap year - it gave me so much more } \\
\text { knowledge of myself, the world, and people }\end{array}$ & 6 & 13 & - & 6 & 4 \\
\hline Moving events & $\begin{array}{l}\text { "When I was } 15 \text { years old I moved to Australia indefinitely - I struggled to be } \\
\text { myself in a new environment" }\end{array}$ & 10 & 7 & 4 & 24 & 4 \\
\hline $\begin{array}{l}\text { Mortality/life } \\
\text { threatening events }\end{array}$ & $\begin{array}{l}\text { When I was } 20 \text { I had an horrible car accident - I learnt that you should always try } \\
\text { to live your life as best as possible" }\end{array}$ & 15 & - & 32 & 10 & 27 \\
\hline Educational events & "When I was 13 I received the highest grades for my year level" & 16 & 25 & 8 & 14 & 6 \\
\hline Sporting events & $\begin{array}{l}\text { "my cricket team won the National Championships - it showed me that hard work } \\
\text { can actually pay off" }\end{array}$ & 7 & 13 & 2 & 6 & N/A \\
\hline Holiday events & "One Christmas we stayed at the beach - It was so hot all we did was swim" & 2 & 5 & - & - & N/A \\
\hline Other achievements & $\begin{array}{l}\text { "my band released our first EP. It made me love music even more and gave me a } \\
\text { taste of what it would be like to be in a famous band" }\end{array}$ & 2 & 5 & - & - & N/A \\
\hline $\begin{array}{l}\text { Mental health } \\
\text { events }\end{array}$ & $\begin{array}{l}\text { "I was } 17 \text { I developed bulimia. - I believe it happened for a reason, and I can now } \\
\text { sympathise and help others facing the same issues. }\end{array}$ & 4 & - & 7 & 4 & N/A \\
\hline Identity events & $\begin{array}{l}\text { "I decided I was gay - I had been really conflicted for ages and very indecisive - I } \\
\text { know who I am now }\end{array}$ & 1 & 1 & - & 5 & N/A \\
\hline Other* & "When I was 17 years old I got arrested for disorderly behaviour" & 12 & 11 & 9 & 8 & 12 \\
\hline
\end{tabular}

* The 'Other' category includes events such as getting into trouble, bullying

N/A $=$ Not Assessed 


\section{Appendix B}

\section{Participants' Response to the Life Story Task}

After completing the autobiographical narrative task, participants from Study 2 (Chapter Six) completed an additional two questions to assess their reaction to completing the task. The decision to include these questions was based on anecdotal observations of participants' reactions and comments made in relation to the task. When conducting Study 1, many participants commented at the end of the session, that the task was a positive experience for them. These participants noted that writing about their experiences helped them to process the event, and that doing so was "therapeutic". While no participants mentioned that the task was a negative experience, the positive comments made by many led me to question whether this would be the case for all individuals. To assess this, two questions were added to the end of the autobiographical narrative task in Study 2.

These two questions assessed participants subjective assessments regarding their enjoyment of the life story task and the impact the task had on their feelings about themselves. In the first question, participants were asked "How positive or negative was your experience of completing this [the life story] task?". This question was labelled as a measure of 'enjoyment'. Participants responded on a 5-point Likert scale ( $1=$ extremely positive, $2=$ somewhat positive, $3=$ neither positive or negative, $4=$ somewhat negative, $5=$ extremely negative). In the second question, participants were asked "How did this task make you feel about yourself?". This question was labelled as a measure of 'impact'. Participants again responded on a 5-point Likert scale $(1=$ made me feel really good about myself, $2=$ made me feel quite good about myself, 3 = didn't make me feel good or bad about myself, $4=$ made me feel quite bad about myself, 5 = made me feel really bad about myself).

For ease of analysis, the two questions measuring enjoyment and impact of the life story task were reverse-scored so that higher scores reflected a more positive response. Table B1 presents participants ratings for each of these questions. Of note, just under three-quarters of participants reported that completing the autobiographical narrative task was a positive experience $(72 \%)$ and that completing the task made them feel good about themselves $(73 \%)$. In contrast, approximately one in ten participants reported that completing the task was a negative experience (10\%) and that completing the task made them feel bad about themselves $(9 \%)$. The remainder of participants reported that completing the task was neither positive 
nor negative $(18 \%)$ and that the task did not make them feel neither bad nor good about themselves $(18 \%)$.

\section{Table B1}

Participants' experience of the life story narrative task

How positive or negative was your experience of completing this [the life story] task?

n $\%$

Extremely positive

Somewhat positive

Neither positive or negative

Somewhat negative

Extremely negative

How did this task make you feel about yourself?

Made me feel really good about myself

Made me feel quite good about myself

Didn't make me feel good or bad about myself

Made me feel quite bad about myself

Pearson correlations then examined associations between participants' subjective assessments regarding their 'enjoyment' and the 'impact' of the task and the number of positive or negative self-event connections found in life story narratives constructed in the task. Of note, participants who made higher numbers of negative self-event connections across narratives of high, low and turning points (combined), were significantly more likely to report that they found the task to be a negative experience, and were more likely to report 
that completing the task made them feel negatively about themselves. The number of positive self-event connections found in narratives of high, low and turning points (combined) were unrelated to participants' ratings of enjoyment and impact of the task.

Table B2

Correlations amongst participants' subjective assessments of the enjoyment and impact of the autobiographical narrative task, and positive and negative self-event connections

$\begin{array}{llll}1 & 2 & 3 & 4\end{array}$

1. Enjoyment

2. Impact $.63^{* *}-$

3. Positive self-event connections $.15-.08$

4. Negative self-event connections $-.22 * * \quad-.34 * * \quad-.10$

These findings have clear implications for research that employs autobiographical narrative tasks of a similar nature to that used in the studies presented in this thesis. These findings show that for some young adults, the experience of completing the autobiographical narrative task was aversive and had a negative impact on their feelings about themselves. The ethical implications of these findings are discussed in more detail in Chapter 8: Discussion. It is important to note, however, that researchers employing autobiographical narrative tasks in the future should be aware of possible harm to participants who may find such tasks aversive, and to mitigate these risks where possible. 


\section{References}

Abela, J. R. Z., Auerbach, R. P., Sarin, S., \& Lakdawalla, Z. (2009). Core beliefs and history of Major Depressive Episodes in currently non-depressed university students. Cognitive Therapy and Research, 33(1), 50-58. doi: 10.1007/s10608-008-9185-y

Abramson, L. Y., Seligman, M. E., \& Teasdale, J. D. (1978). Learned helplessness in humans: Critique and reformulation. The Journal of Abnormal Psychology, 87(1), 4974. doi: 10.1037/0021-843X.87.1.49

Adler, J. M., Kissel, E. C., \& McAdams, D. P. (2006). Emerging from the CAVE: Attributional style and the narrative study of identity in midlife adults. Cognitive Therapy and Research, 30(1), 39-53. doi: 10.1007/s10608-006-9005-1

Adler, J. M., \& Poulin, M. J. (2009). The political is personal: Narrating 9/11 and psychological well-being. Journal of Personality, 77(4), 903-932. doi: 10.1111/j.1467-6494.2009.00569.x

Aiken, L. S., \& West, S. G. (1991). Multiple regression: Testing and interpreting interactions. Newbury Park, CA: Sage Publications.

Alloy, L. B., Abramson, L. Y., Whitehouse, W. G., Hogan, M. E., Tashman, N. A., Steinberg, D. L., . . Donovan, P. (1999). Depressogenic cognitive styles: predictive validity, information processing and personality characteristics, and developmental origins. Behaviour Research and Therapy, 37(6), 503-531. doi: 10.1016/S00057967(98)00157-0

Angus, L., Levitt, H., \& Hardtke, K. (1999). The narrative processes coding system: Research applications and implications for psychotherapy practice. Journal of Clinical Psychology, 55(10), 1255-1270. doi: 10.1002/(SICI)10974679(199910)55:10<1255::AID-JCLP7>3.0.CO;2-F

Arnett, J. J. (2000). Emerging adulthood: A theory of development from the late teens through the twenties. American Psychologist, 55(5), 469-480. doi: 10.1037//0003066X.55.5.469 
Arnett, J. J. (2007). Emerging adulthood: What is it, and what is it good for? Child Development Perspectives, 1(2), 68-73. doi: 10.1111/j.1750-8606.2007.00016.x

Baddeley, J. L., \& Pennebaker, J. W. (2011). A postdeployment expressive writing intervention for military couples: A randomized controlled trial. Journal of Traumatic Stress, 24(5), 581-585. doi: 10.1002/jts.20679

Baker-Ward, L. E., Eaton, K. L., \& Banks, J. B. (2005). Young soccer players' reports of a tournament win or loss: Different emotions, different narratives. Journal of Cognition and Development, 6(4), 507-527. doi: 10.1207/s15327647jcd0604_4

Barnes-Holmes, D., Hayes, S. C., \& Dymond, S. (2001). Self and self-directed rules. In S. C. Hayes, D. Barnes-Holmes, \& B. Roche (Eds.), Relational Frame Theory: A PostSkinnerian Account of Human Language and Cognition (pp 129-139). New York:Plenum.

Barnes-Holmes, Y., Hayes, S. C., Barnes-Holmes, D., \& Roche, B. (2001). Relational frame theory: A post-skinnerian account of human language and cognition. Advances in Child Development and Behavior, 28, 101-138. doi: 10.1016/S0065-2407(02)80063-5

Baron, R. M., \& Kenny, D. A. (1986). The moderator-mediator variable distinction in social psychological research: Conceptual, strategic, and statistical considerations. Journal of Personality and Social Psychology, 51(6), 1173-1182. doi: 10.1037/00223514.51.6.1173

Bauer, J. J., McAdams, D. P., \& Pals, J. L. (2008). Narrative identity and eudaimonic wellbeing. Journal of Happiness Studies, 9(1), 81-104. doi: 10.1007/s10902-006-9021-6

Bauer, J. J., McAdams, D. P., \& Sakaeda, A. R. (2005). Interpreting the good life: Growth memories in the lives of mature, happy people. Journal of Personality and Social Psychology, 88(1), 203-217. doi: 10.1037/0022-3514.88.1.203

Bauer, P. J., Stennes, L., \& Haight, J. C. (2003). Representation of the inner self in autobiography: Women's and men's use of internal states language in personal narratives. Memory, 11(1), 27-42. doi: 10.1080/09658210143000443 
Baumeister, R. F., Campbell, J. D., Krueger, J. I., \& Vohs, K. D. (2003). Does high selfesteem cause better performance, interpersonal success, happiness, or healthier lifestyles? Psychological Science in the Public Interest, 4(1), 1-44. doi:10.1111/15291006.01431

Baumeister, R., \& Wilson, B. (1996). Life stories and the four needs for meaning. Psychological Inquiry, 7(4), 322-377. doi: 10.1207/s15327965pli0704_2

Baumeister, R. F., \& Newman, L. S. (1994). How stories make sense of personal experiences: Motives that shape autobiographical narratives. Personality and Social Psychology Bulletin, 20(6), 676-690. doi: 10.1177/0146167294206006

Bech, P., Olsen, L. R., Kjoller, M., \& Rasmussen, N. K. (2003). Measuring well-being rather than the absence of distress symptoms: a comparison of the SF-36 Mental Health subscale and the WHO-Five Well-Being Scale. International Journal of Methods in Psychiatric Research, 12(2), 85-91. doi: 10.1002/mpr.145

Beck, A. T., \& Clark, D. A. (1997). An information processing model of anxiety: Automatic and strategic processes. Behaviour Research and Therapy, 35(1), 49-58. doi: $10.1016 / \mathrm{S} 0005-7967$

Beck, J. S. (1995). Cognitive Therapy: Basics and Beyond. New York, NY: The Guilford Press.

Bentall, R. P., Kinderman, P., \& Bowen-Jones, K. (1999). Response latencies for the causal explanations of depressed, paranoid, and normal individuals: Availability of selfrepresentations. Cognitive Neuropsychiatry, 4(2), 107-118. doi: $10.1080 / 135468099395990$

Bergouignan, L., Lemogne, C., Foucher, A., Longin, E., Vistoli, D., Allilaire, J.-F., \& Fossati, P. (2008). Field perspective deficit for positive memories characterizes autobiographical memory in euthymic depressed patients. Behaviour Research and Therapy, 46. doi: 10.1016/j.brat.2007.12.007

Berntsen, D., \& Rubin, D. C. (2004). Cultural life scripts structure recall from autobiographical memory. Memory \& Cognition, 32(3), 427-442. doi: 10.3758/BF03195836 
Berntsen, D., \& Rubin, D. C. (2006). The centrality of event scale: A measure of integrating a trauma into one's identity and its relation to post-traumatic stress disorder symptoms. Behaviour Research and Therapy, 44(2), 219-231. doi: 10.1016/j.brat.2005.01.009

Berntsen, D., \& Rubin, D. C. (2006b). Emotion and vantage point in autobiographical memory. Cognition \& Emotion, 20(8), 1193-1215. doi: 10.1080/02699930500371190

Berntsen, D., \& Rubin, D. C. (2007). When a trauma becomes a key to identity: Enhanced integration of trauma memories predicts posttraumatic stress disorder symptoms. Applied Cognitive Psychology, 21(4), 417-431. doi: 10.1002/acp.1290

Berntsen, D., Rubin, D. C., \& Siegler, I. (2011). Two versions of life: Emotionally negative and positive life events have different roles in the organisation of life story and identity. Emotion, 11(5), 1190-1201. doi: 10.1037/a0024940

Berntsen, D., Willert, M., \& Rubin, D. C. (2003). Splintered memories or vivid landmarks? Qualities and organization of traumatic memories with and without PTSD. Applied Cognitive Psychology, 17(6), 675-693. doi: 10.1002/acp.894

Bird, A., \& Reese, E. (2006). Emotional reminiscing and the development of an autobiographical self. Developmental Psychology, 42(4), 613-626. doi: 10.1037/00121649.42 .4 .613

Blagov, P. S., \& Singer, J. A. (2004). Four dimensions of self-defining memories (specificity, meaning, content, and affect) and their relationships to self-restraint, distress, and repressive defensiveness. Journal of Personality, 72(3), 481-511. doi: 10.1111/j.00223506.2004.00270.x

Blaney, P. H. (1986). Affect and memory: A review. Psychological Bulletin, 99(2), 229-246. doi: 10.1037/0033-2909.99.2.229

Bluck, S., \& Alea, N. (2009). Thinking and talking about the past: Why remember? Applied Cognitive Psychology, 23(8).doi: 10.1002/acp.1612

Bluck, S., \& Habermas, T. (2000). The life story schema. Motivation and Emotion, 24(2), 121-147. doi: 10.1023/A:1005615331901 
Bluck, S., \& Habermas, T. (2001). Extending the study of autobiographical memory: Thinking back about life across the life span. Review of General Psychology, 5(2), 135-147. doi: 10.1037//1089-2680.5.2.135

Boals, A. (2010). Events that have become central to identity: Gender differences in the Centrality of Events Scale for positive and negative events. Applied Cognitive Psychology, 24(1), 107-121. doi: 10.1002/acp.1548

Boals, A. (2012). Improving our understanding of PTSD: The role of event centrality. Paper presented at the 7th Autobiographical Memory and Psychopathology Meeting, Aarhus, Denmark.

Bohanek, J. G., Fivush, R., \& Walker, E. (2005). Memories of positive and negative emotional events. Applied Cognitive Psychology, 19(1), 51-66. doi: 10.1002/acp.1064

Bohn, A., \& Berntsen, D. (2008). Life story development in childhood: The development of life story abilities and the acquisition of cultural life scripts from late middle childhood to adolescence. Developmental Psychology, 44(4), 1135-1147. doi: $10.1037 / 0012-1649.44 .4 .1135$

Brooks, E., \& Dallos, R. (2009). Exploring young women's understandings of the development of difficulties: A narrative biographical analysis. Clinical Child Psychology and Psychiatry, 14(1), 101-115. doi: 10.1177/1359104508100139

Carver, C. S., Scheier, M. F., \& Weintraub, J. K. (1989). Assessing coping strategies: A theoretically based approach. Journal of Personality and Social Psychology, 56(2), 267-283. doi: 10.1037/0022-3514.56.2.267

Chang, E., \& Sanna, L. (2007). Affectivity and psychological adjustment across two adult generations: Does pessimistic explanatory style still matter? Personality and Individual Differences, 43(5), 1149-1159. doi: 10.1016/j.paid.2007.03.007

Ciesla, J. A., \& Roberts, J. E. (2007).Rumination, negative cognition, and their interactive effects on depressed mood.Emotion, 7(3), 555-565. doi: 10.1037/1528-3542.7.3.555 
Conway, M. A., Singer, J. A., \& Tagini, A. (2004). The self and autobiographical memory: correspondence and coherence. Social Cognition, 22(5), 491-529. doi: $10.1521 /$ soco.22.5.491.50768

Cowen, E. L. (1991).In pursuit of wellness.American Psychologist, 46(4), 404-408. doi: 10.1037/0003-066X.46.4.404

Crawley, S., \& French, C. (2005). Field and observer viewpoint in remember-know memories ofpersonal childhood events. Memory, 13(7), 673-681. doi: $10.1080 / 09658210444000296$

Crawford, J. R., \& Henry, J. D. (2003). The depression anxiety stress scales (DASS): Normative data and latent structure in a large non-clinical sample. British Journal of Clinical Psychology, 42(2), 111-131.doi:10.1348/014466503321903544

D'Argembeau, A., \& Van der Linden, M. (2004). Phenomenal characteristics associated with projecting oneself back into the past and forward into the future: Influence of valence and temporal distance. Consciousness and Cognition, 13(4), 844-858. doi: 10.1016/j.concog.2004.07.007

D'Argembeau, A., Van der Linden, M., d'Acremont, M., \& Mayers, I. (2006).Phenomenal characteristics of autobiographical memories for social and non-social events in social phobia.Memory, 14(5), 637-647. doi: 10.1080/09658210600747183

Davis, P. A., \& Burns, G. L. (1999). Influence of emotional intensity and frequency of positive and negative events on depression. European Journal of Psychological Assessment, 15(2), 106-116. doi: 10.1027//1015-5759.15.2.106

Davis, C. G., Nolen-Hoeksema, S., \& Larson, J. (1998). Making sense of loss and benefiting from the experience: Two construals of meaning. Journal of Personality and Social Psychology, 75(2), 561-574. doi: 10.1037/0022-3514.75.2.561

De Graaf, L. E., Roelofs, J., \& Huibers, M. J. H. (2009).Measuring dysfunctional attitudes in the general population: The Dysfunctional Attittude Scale (form A) Revised.Cognitive Therapy and Research, 33 , 345-355. doi:10.1007/s10608-009-9229-y 
Diener, E., Emmons, R. A., Larsen, R. J., \& Griffen, S. (1985). The Satisfaction With Life Scale. Journal of Personality Assessment, 49(1), 71-75. doi: 10.1207/s15327752jpa4901_13

Digman, J. M. (1990). Personality structure: Emergence of the five-factor model. Annual Review of Psychology, 41, 417-440. doi: 10.1146/annurev.ps.41.020190.002221

Donaldson, C., \& Lam, D. (2004).Rumination, mood and social problem-solving in major depression.Psychological Medicine, 34(7), 1309-1318. doi: $10.1017 / \mathrm{S} 0033291704001904$

Erikson, E. H. (1968).Identity, youth and crisis. New York: W. W. Norton Company.

Fivush, R. (2007). Maternal reminiscing style and children's developing understanding of self and emotion.Clinical Social Work Journal, 35(1), 37-46. doi: 10.1007/s10615-0060065-1

Fivush, R. (2011). The Development of Autobiographical Memory.Annual review of psychology, 62(1), 559-582.doi: 10.1146/annurev.psych.121208.131702

Fivush, R., Berlin, L. J., McDermott Sales, J., Mennuti-Washburn, J., \& Cassidy.(2003). Functions of parent-child reminiscing about emotionally negative events.Memory, 11(2), 179-192. doi: 10.1080/741938209

Fivush, R. and J. Buckner (2003).Constructing Gender and Identity through Autobiographical Narratives.In R. Fivush and C. Haden (eds) Autobiographical Memory and the Construction of a Narrative Self: Developmental and Cultural Perspectives, pp. 149-67. Hillsdale, NJ: Erlbaum.

Fivush, R., Brotman, M. A., Buckner, J. P., \& Goodman, S. H. (2000). Gender differences in parent-child emotion narratives. Sex Roles, 42(3/4), 233-253. doi:

10.1023/A:1007091207068

Fivush, R., Hazzard, A., McDermott Sales, J., Sarfati, D., \& Brown, T. (2003). Creating coherence out of chaos? Children's narratives of emotionally positive and negative events.Applied Cognitive Psychology, 17(1), 1-19.doi: 10.1002/acp.854 
Fivush, R., Marin, K., Crawford, M., Reynolds, M., \& Brewin, C. R. (2007).Children's narratives and well-being.Cognition \& Emotion, 21(7), 1414-1434. doi: $10.1080 / 02699930601109531$

Fivush, R., \& Nelson, K. (2006). Parent-child reminiscing locates the self in the past. The British Journal of Developmental Psychology, 24(1), 235-251. doi: $10.1348 / 026151005 \times 57747$

Fivush, R., Reese, E., \& Haden, C. A. (2006). Elaborating on Elaborations: Role of Maternal Reminiscing Style in Cognitive and Socioemotional Development. Child Development, 77(6), 1568-1588. doi: 10.1111/j.1467-8624.2006.00960.x

Fletcher, L., \& Hayes, S.C. (2005). Relational frame theory, acceptance and commitment therapy, and a functional analytic definition of mindfulness. Journal of Rational-

Emotive \& Cognitive Therapy, 23(4),.doi:315-336.10.1007/s10942-005-0017-7

Folkman, S., \& Lazarus, R. S. (1980).An analysis of coping in a middle-aged community sample.Journal of Health and Social Behavior, 21, 219-239.

Folkman, S., Lazarus, R. S., Gruen, R. J., \& DeLongis, A. (1986).Appraisal, coping, health status, and psychological symptoms.Journal of Personality and Social Psychology, 50, 571-579.

Frank, M. G., \& Gilovich, T. (1989).Effect of memory perspective on retrospective causal attributions.Journal of Personality and Social Psychology, 57(3), 399-403. doi: 10.1037/0022-3514.57.3.399

Fresco, D. M., Alloy, L. B., \& Reilly-Harrington, N. (2006).Association of attributional style for negative and positive events and the occurrence of life events with depression and anxiety.Journal of Social and Clinical Psychology, 25(10), 1140-1160. doi: $10.1521 /$ jscp.2006.25.10.1140

Gara, M. A., Woolfolk, R. L., Cohen, B. D., Goldston, R. B., Allen, L. A., \& Novalany, J. (1993). Perception of self and other in major depression. Journal of Abnormal Psychology, 102(1), 93-100. doi:10.1037/0021-843X.102.1.93 
Gillies, J., \& Neimeyer, R. A. (2006). Loss, grief, and the search for significance: Toward a model of meaning reconstruction in bereavement. Journal of Constructivist Psychology, 19(1), 31-65. doi: 10.1080/10720530500311182

Gortner, E.-M., Rude, S. S., \& Pennebaker, J. W. (2006).Benefits of expressive writing in lowering rumination and depressive symptoms.Behavior Therapy, 37(3), 292-303. doi: 10.1016/j.beth.2006.01.004

Grisham, J. R., Flower, K. N., Williams, A. D., \& Moulds, M. L. (2011).Reappraisal and rumination during recall of a sad memory.Cognitive Therapy and Research, 35(3), 276-283. doi: 10.1007/s10608-009-9288-0

Gross, J. J. (1998). The emerging field of emotion regulation: An integrative review. Review of General Psychology, 2(3), 271-299. doi: 10.1037/1089-2680.2.3.271

Gross, J. J., \& John, O. P. (2003). Individual differences in two emotion regulation processes: implications for affect, relationships, and well-being. Journal of Personality and Social Psychology, 85(2), 348-362. doi: 10.1037/0022-3514.85.2.348

Grossbaum, M. F., \& Bates, G. W. (2002).Correlates of psychological well-being at midlife: The role of generativity, agency and communion, and narrative themes. International Journal of Behavioral Development, 26(2), 120-127. doi: $10.1080 / 01650250042000654$

Grysman, A., \& Hudson, J. (2010). Abstracting and extracting: Causal coherence and the development of the life story. Memory, 18(6), 565-580. doi: $10.1080 / 09658211.2010 .493890$

Habermas, T. (2007). How to tell a life: The development of the cultural concept of biography. Journal of Cognition and Development, 8(1), 1-31. doi: $10.1080 / 15248370709336991$

Habermas, T. (2010). Autobiographical Reasoning: Arguing and narrating from a biographical perspective. In T. Habermas (Ed.), The development of autobiographical 
reasoning in adolescence and beyond. New Directions for Child and Adolescent Development(Vol. 131, pp. 131, 1-17).

Habermas, T., \& Bluck, S. (2000).Getting a life: the emergence of the life story in adolescence.Psychological Bulletin, 126(5), 748-769. doi: 10.1037OT0332909.126.5.741

Habermas, T., \& de Silveira, C. (2008). The development of global coherence in life narratives across adolescence: temporal, causal, and thematic aspects. Developmental Psychology, 44(3), 707-721. doi: 10.1037/0012-1649.44.3.707

Habermas, T., Ott, L. M., Schubert, M., Schneider, B., \& Pate, A. (2008).Stuck in the past: negative bias, explanatory style, temporal order, and evaluative perspectives in life narratives of clinically depressed individuals.Depression and Anxiety, 25(11), 121132. doi: 10.1002/da.20389

Habermas, T., \& Paha, C. (2001).The development of coherence in adolescents' life narratives Narrative Inquiry, 11(1), 35-54.doi: 10.1075/ni.11.1.02hab

Haden, C. A., Ornstein, P. A., Rudek, D. J., \& Cameron, D. (2009). Reminiscing in the early years: patterns of maternal elaborativeness and children's remembering. International Journal of Behavioral Development, 33(2), 118-130. doi:

$10.1177 / 0165025408098038$

Hankin, B. L., Abramson, L. Y., Moffitt, T. E., Silva, P. A., McGee, R., \& Angell, K. A. (1998). Development of depression from preadolescence to young adulthood: Emerging gender differences in a 10 year longitudinal study. Journal of Abnormal Psychology, 107(1), 128-140. doi: 10.1037/0021-843X.107.1.128

Harrington, R., \& Loffredo, D. A. (2011).Insight, rumination, and self-reflection as predictors of well-being.The Journal of Psychology: Interdisciplinary and Applied, 145(1), 3957. doi: 10.1080/00223980.2010.528072

Hayes, S. C., Barnes-Holmes, D., \& Roche, B. (Eds.). (2001). Relational Frame Theory: A Post-Skinnerian account of human language and cognition. New York: Plenum Press. 
Hayes, S. C., Strosahl, K., \& Wilson, K. G. (1999).Acceptance and Commitment Therapy: An experiential approach to behavior change. New York: Guilford Press

Headey, B., \& Wearing, A. (1989). Personality, life events, and subjective well-being: Toward a dynamic equilibrium model. Journal of Personality and Social Psychology, 57(4), 731-739. doi: 10.1037/0022-3514.57.4.731

Hill, K., \& Dallos, R. (2012). Young people's stories of self-harm: A narrative study. Clinical Child Psychology and Psychiatry, 17(3), 459-475. doi: 10.1177/1359104511423364

Hilt, L. M., \& Pollak, S. D. (2012).Getting out of rumination: Comparison of three brief interventions in a sample of youth.Journal of Abnormal Child Psychology, 40(7), 1157-1165. doi: 10.1007/s10802-012-9638-3

Ho, S. M. Y., Chan, M. W. Y., Yau, T. K., \& Yeung, R. M. W. (2011). Relationships between explanatory style, posttraumatic growth and posttraumatic stress disorder symptoms among Chinese breast cancer patients. Psychology and Health, 26(3), 269285. doi: 10.1080/08870440903287926

Holmes, E. A., Coughtrey, A. E., \& Connor, A. (2008). Looking at or through rose-tinted glasses? Imagery perspective and positive mood.Emotion, 8(6), 875-879. doi: $10.1037 / \mathrm{a} 0013617$

Honos-Webb, L., Harrick, E. A., Stiles, W. B., \& Park, C. L. (2000). Assimilation of traumatic experiences and physical-health outcomes: Cautions for the Pennebaker paradigm. Psychotherapy: Theory/Research/Practice/Training, 37(4), 307-314. doi: 10.1037/0033-3204.37.4.307

Howard, A. L., Galambos, N. L., \& Krahn, H. J. (2010).Paths to success in young adulthood from mental health and life transitions in emerging adulthood.International Journal of Behavioral Development, 34(6), 538-546. doi: 10.1177/0165025410365803

Johnson, J. G., Han, Y.-S., Douglas, C. J., Johannet, C. M., \& Russell, T. (1998). Attributions for positive life events predict recovery from depression among psychiatric inpatients: An investigation of the needles and abramson model of recovery from depression. Journal of Consulting and Clinical Psychology, 66(2), 369-376. doi: 10.1037/0022006X.66.2.369 
Joiner, T. E., \& Wagner, K. D. (1995). Attributional style and depression in children and adolescents: A meta-analytic review. Clinical Psychology Review, 15(8), 777-798. doi: 10.1016/0272-7358(95)00046-1

Jose. P. E. (2004) ModGraph: An internet-based statistical program to graphically display statistical moderation. New Zealand: Victoria University of Wellington. Retrieved November 22, 2010, from http://www.vuw.ac.nz/psyc/staff/paul-jose/index.aspx.

Kaluza, G. (2000). Changing unbalanced coping profiles-A prospective controlled intervention trial in worksite health promotion. Psychology \& Health, 15(3), 423-433. doi: 10.1080/08870440008402003

Kanter, J. W., Busch, A. M., Weeks, C. E., \& Landes, S. J. (2008). The nature of clinical depression: Symptoms, syndromes, and behavior analysis. The Behavior Analyst, $31(1), 1-21$.

Kashdan, T., Biwas-Diener, R., \& King, L. A. (2008).Reconsidering happiness: The costs of distinguishing between hedonics and eudaimonia.The Journal of Positive Psychology: Dedicated to furthering research and promoting good practice, 3(4), 219-233. doi: $10.1080 / 17439760802303044$

King, L. A., Hicks, J. A., Krull, J. L., \& Del Gaiso, A. K. (2006). Positive affect and the experience of meaning in life.Journal of Personality and Social Psychology, 90(1), 179-196. doi: 10.1037/0022-3514.90.1.179

King, L. A., Scollon, C. K., Ramsey, C., \& Williams, T. (2000). Stories of life transition: Subjective well-being and ego development in parents of children with Down Syndrome. Journal of Research in Personality, 34(4), 509-536. doi: 10.1006/jrpe.2000.2285

Korkeila, J., Vahtera, J., Nabi, H., Kivimaki, M., Korkeila, K., Sumanen, M., . . Koskenvuo, M. (2010). Childhood adversities, adulthood life events and depression.Journal of Affective Disorders, 127(1), 130-138. doi: 10.1016/j.jad.2010.04.031

Kuyken, W., \& Howell, R. (2006).Facets of autobiographical memory in adolescents with major depressive disorder and never-depressed controls.Cognition and Emotion, 20(3), 466-487. doi: 10.1080/02699930500342639 
Kuyken, W., \& Moulds, M. L. (2009). Remembering as an observer: How is autobiographical memory retrieval vantage perspective linked to depression? Memory, 17(6), 624-634. doi: 10.1080/09658210902984526

Laidlaw, K., \& Davidson, K. M. (2001). The personal nature of depression: Assessing the operation of self-schema in depression. Clinical Psychology and Psychotherapy, 8(2), 97-105. doi: 10.1002/cpp.282

LaNoue, M., Graeber, D., de Hernandez, B. U., Warner, T. D., \& Helitzer, D. (2012).Direct and indirect effects of childhood adversity on adult depression.Community Mental Health Journal, 48(2), 187-192. doi: 10.1007/s10597-010-9369-2

Lazarus, R. S. (1991). Progress on a cognitive-motivational-relational theory of emotion.American Psychologist, 46(8), 819-834. doi: 10.1037/0003-066X.46.8.819

Lazarus, R. S. (1993a). Coping theory and research: Past, present, and future. Psychosomatic Medicine, 55(3), 234-247.

Lazarus, R. S. (1993b). From psychological stress to the emotions: A history of changing outlooks. Annual Review of Psychology, 44, 1-21. doi: 10.1146/annurev.ps.44.020193.000245

Lazarus, R. S. (1995). Cognition and emotion from the RET viewpoint. Journal of RationalEmotive and Cognitive Behaviour Therapy, 13(1), 29-54. doi: 10.1007/BF02354556

Lazarus, R. S., \& Folkman, S. (1984). Psychological stress and the coping process. New York, NY: Springer.

Lemogne, C., Piolino, P., Friszer, S., Claret, A., Girault, N., Jouvent, R., . . Fossati, P. (2006). Episodic autobiographical memory in depression: Specificity, autonoetic consciousness, and self-perspective. Consciousness and Cognition, 15(2), 258-268. doi: 10.1016/j.concog.2005.07.005

Libby, L. K., \& Eibach, R. P. (2011).Self-enhancement or self-coherence? Why people shift visual perspective in mental images of the personal past and future. Personality and Social Psychology Bulletin, 37(5), 714-726. doi: 10.1177/0146167211400207 
Libby, L. K., Eibach, R. P., \& Gilovich, T. (2005). Here's looking at me: The effect of memory perspective on assessments of personal change. Journal of Personality and Social Psychology, 88(1), 50-62. doi: 10.1037/0022-3514.88.1.50

Lilgendahl, J. P., \& McAdams, D. P. (2011).Constructing stories of self-growth: how individual differences in patterns of autobiographical reasoning relate to well-being in midlife.Journal of Personality, 79(2), 391-428. doi: 10.1111/j.14676494.2010.00688.x

Linde, C. (1993). Life stories: The Creation of Coherence. Oxford, England: Oxford University Press.

Loftus, E. F., \& Fathi, D. C. (1985).Retreiving multiple autobiographical memories.Social Cognition, 3(3), 280-295. doi: 10.1521/soco.1985.3.3.280

Lovibond, S. H., \& Lovibond, P. F. (1995).Manual for the Depression Anxiety Stress Scales. Sydney, New South Wales, Australia: The Psychology Foundation of New South Wales Inc, University of New South Wales.

Luten, A. G., Ralph, J. A., \& Mineka, S. (1997). Pessimistic attributional style: Is it specific to depression versus anxiety versus negative affect? Behaviour Research and Therapy, 35(8), 703-719. doi: 10.1016/S0005-7967

Lyubomirsky, S., Sousa, L., \& Dickerhoof, R. (2006).The costs and benefits of writing, talking, and thinking about life's triumphs and defeats.Journal of Personality and Social Psychology, 90(4), 692-708. doi: 10.1037/0022-3514.90.4.692

MacCallum, F., \& Bryant, R. (2008).Self-defining memories in complicated grief.Behaviour Research and Therapy, 46(12), 1311-1315. doi: 10.1016/j.brat.2008.09.003

Malle, B. F., \& Horowitz, L. M. (1995). The puzzle of negative self-views: An exploration using the schema concept. Journal of Personality and Social Psychology, 68(3), 470484. doi: 10.1037/0022-3514.68.3.470

Mansell, W., \& Clark, D. M. (1999). How do I appear to others? social anxiety and processing of the observable self. Behaviour Research and Therapy, 37(5), 419-434. doi:10.1016/S0005-7967(98)00148-X 
Marks, A. D., Sobanski, D. J., \& Hine, D. W. (2010). Do dispositional rumination and/or mindfulness moderate the relationship between life hassles and psychological dysfunction in adolescents? Australian and New Zealand Journal of Psychiatry, 44(9), 831-838. doi: 10.3109/00048674.2010.487478

Marsh, E., \& Tversky, B. (2004).Spinning the stories of our lives.Applied Cognitive Psychology, 18(5), 491-503.doi: 10.1002/acp.1001

Mauss, I. B., Cook, C. L., Cheng, J. Y. J., \& Gross, J. J. (2007). Individual differences in cognitive reappraisal: experiential and physiological responses to an anger provocation. International Journal of Psychophysiology, 66(2), 116-124. doi: 10.1016/j.ijpsycho.2007.03.017

McAdams, D. P. (1988).Power, intimacy, and the life story. New York: The Guilford Press.

McAdams, D. P. (1993).The stories we live by: Personal myths and the making of the self. New York: The Guilford Press.

McAdams, D. P. (1995). What do we know when we know a person? Journal of Personality, 63(3), 365-396. doi: 10.1111/j.1467-6494.1995.tb00500.x

McAdams, D. P. (1996). Personality, modernity, and the storied self: A contemporary framework for studying persons. Psychological Inquiry: An International Journal for the Advancement of Psychological Theory, 7(4), 295-321. doi:

10.1207/s15327965pli0704_1

McAdams, D. P. (2006).Guided Autobiography Retrieved January, 2010, from http://www.sesp.northwestern.edu/foley/instruments/guided/

McAdams, D. P. (2001).The psychology of life stories.Review of General Psychology, 5(2), 100-122. doi: 10.1037//I089-2680.5.2.100

McAdams, D. P. (2011).Narrative Identity. In S. J. Schwartz, K. Luyckx \& V. L. Vignoles (Eds.), Handbook of Identity Theory and Research: Volume 1 Structures and Processes (pp. 99-115). New York: Springer. 
McAdams, D. P., \& Pals, J. L. (2006). A new big five: Fundamental principles for an integrative science of personality. American Psychologist, 61(3), 204-217. doi: 10.1037/0003-066X.61.3.204

McAdams, D. P., Reynolds, J., Lewis, M., Patten, A. H., \& Bowman, P. (2001). When bad things turn good and good things turn bad: Sequences of redemption and contamination in life narrative and their relation to psychosocial adaptation in midlife adults and in students. Personality and Social Psychology Bulletin, 27(4), 474-485. doi: $10.1177 / 0146167201274008$

McIsaac, H. K., \& Eich, E. (2004).Vantage point in traumatic memory.Psychological Science, 15(4), 248-253. doi: 10.1111/j.0956-7976.2004.00660.x

McLaughlin, K. A., Borkovec, T. D., \& Sibrava, N. J. (2007). The effects of worry and rumination on affect states and cognitive activity. Behavior Therapy, 38(1), 23-38. doi: 10.1016/j.beth.2006.03.003

McLaughlin, K. A., \& Nolen-Hoeksema, S. (2011). Rumination as a transdiagnostic factor in depression and anxiety.Behaviour Research and Therapy, 49(3), 186-193. doi: 10.1016/j.brat.2010.12.006

McLean, K. C. (2005). Late adolescent identity development: Narrative meaning making and memory telling. Developmental Psychology, 41(4), 683-691. doi: 10.1037/00121649.41.4.683

McLean, K. C. (2008). Stories of the young and the old: Personal continuity and narrative identity. Developmental Psychology, 44(1), 254-264. doi: 10.1037/00121649.44.1.254

McLean, K. C., \& Breen, A. V. (2009). Processes and content of narrative identity development in adolescence: Gender and well-being. Developmental Psychology, 45(3), 702-710. doi: 10.1037/a0015207

McLean, K. C., Breen, A. V., \& Fournier, M. A. (2010).Constructing the self in early, middle, and late adolescent boys: Narrative identity, individuation, and wellbeing.Journal of Research on Adolescence, 20(1), 166-187. doi: 10.1111/j.15327795.2009.00633.x 
McLean, K. C., \& Fournier, M. A. (2008).The content and processes of autobiographical reasoning in narrative identity.Journal of Research in Personality, 42(3), 527-545. doi: 10.1016/j.jrp.2007.08.003

McLean, K. C., \& Lilgendahl, J. P. (2008). Why recall our highs and lows: Relations between memory functions, age, and well-being. Memory, 16(7), 751-762. doi: $10.1080 / 09658210802215385$

McLean, K. C., \& Mansfield, C. D. (2010). To reason or not to reason: Is autobiographical reasoning always beneficial? In T. Habermas (Ed.), The development of autobiographical reasoning in adolescence and beyond. New Directions for Child and Adolescent Development(Vol. 131, pp. 85-97).

McLean, K. C., Pasupathi, M., \& Pals, J. L. (2007). Selves creating stories creating selves: A process model of self-development. Personality and Social Psychology Review, 11(3), 262-278. doi: 10.1177/1088868307301034

McLean, K. C., \& Pratt, M. W. (2006). Life's little (and big) lessons: Identity statuses and meaning-making in the turning point narratives of emerging adults. Developmental Psychology, 42(4), 714-722. doi: 10.1037/0012-1649.42.4.714

McLean, K. C., \& Thorne, A. (2003).Late adolescents' self-defining memories about relationships. Developmental Psychology, 39(4), 635-645. doi: 10.1037/00121649.39.4.635

Mellings, T. M. B., \& Alden, L. E. (2000). Cognitive processes in social anxiety: the effects of self-focus, rumination and anticipatory processing. Behaviour Research and Therapy, 38(3), 243-257. doi: 10.1016/S0005-7967(99)00040-6

Mongrain, M., \& Blackburn, S. (2005).Cognitive vulnerability, lifetime risk, and the recurrence of major depression in graduate students.Cognitive Therapy and Research, 29(6), 747-768. doi: 10.1007/s10608-005-4290-7

Moore, S. A., Varra, A. A., Michael, S. T., \& Simpson, T. L. (2010).Stress-related growth, positive reframing, and emotional processing in the prediction of post-trauma functioning among veterans in mental health treatment.Psychological Trauma: Theory, Research, Practice, and Policy, 2(2), 93-96. doi: 10.1037/a0018975 
Morgan, J., \& Farsides, T. (2009).Psychometric evaluation of the meaningful life measure.Journal of Happiness Studies, 10(3), 351-366. doi: 10.1007/s10902-0089093-6

Morris, S. J. (2007). Attributional biases in subclinical depression: A schema-based account. Clinical Psychology and Psychotherapy, 14(1), 32-47. doi: 10.1002/cpp.512

Needles, D. J., \& Abramson, L. Y. (1990). Positive life events, attributional style, and hopefulness: Testing a model of recovery from depression. Journal of Abnormal Psychology, 99(2), 156-165. doi: 10.1037/0021-843X.99.2.156

Nelson, K. (2003). Narrative and self, myth and memory: Emergence of the cultural self. In R. Fivush \& C. A. Haden (Eds.), Autobiographical Memory and the Construction of a Narrative Self: Developmental and Cultural Perspectives (pp. 3-28). Mahwah, NJ: Lawrence Erlbaum Associates, Inc.

Nelson, K., \& Fivush, R. (2004). The emergence of autobiographical memory: A social cultural developmental theory. Psychological Review, 111(2), 486-511. doi: 10.1037/0033-295X.111.2.486

Newby, J. M., \& Moulds, M. L. (2011). Intrusive memories of negative events in depression: Is the centrality of the event important? Journal of Behavior Therapy and Experimental Psychiatry, 42(3), 277-283. doi:10.1016/j.jbtep.2010.12.011

Nezlek, J. B., \& Kuppens, P. (2008).Regulating positive and negative emotions in daily life.Journal of Personality, 76(3), 561-581. doi: 10.1111/j.1467-6494.2008.00496.x

Nigro, G., \& Neisser, U. (1983).Point of view in personal memories.Cognitive Psychology, 15(4), 467-482. doi: 10.1016/0010-0285(83)90016-6

Nolen-Hoeksema, S. (1991).Responses to depression and their effects on the duration of depressive episodes. Journal of Abnormal Psychology, 100(4), 569-582. doi: 10.1037/0021-843X.100.4.569

Nolen-Hoeksema, S., Wisco, B. E., \& Lyubomirsky, S. (2008). Rethinking rumination.Perspectives on Psychological Science, 3(5), 400-424. doi: 10.1111/j.1745-6924.2008.00088.x 
Palker-Corell, A., \& Marcus, D. K. (2004). Partner abuse, learned helplessness, and trauma symptoms. Journal of Social and Clinical Psychology, 23(4), 445-462. doi: 10.1521/jscp.23.4.445.40311

Pals, J. L. (2006). Narrative identity processing of difficult life experiences: Pathways of personality development and positive self-transformation in adulthood. Journal of Personality, 74(4), 1079-1110. doi: 10.1111/j.1467-6494.2006.00403.x

Pals, J. L., \& McAdams, D. P. (2004). The transformed self: A narrative understanding of Posttraumatic Growth. Psychological Inquiry, 15(1), 65-69. doi: 10.1207/s15327965pli1501_02

Park, C. L. (2008). Testing the meaning making model of coping with loss.Journal of Social and Clinical Psychology, 27(9), 970-994. doi: 10.1521/jscp.2008.27.9.970

Park, C. L. (2010). Making sense of the meaning literature: An integrative review of meaning making and its effects on adjustment to stressful life events.Psychological Bulletin, 136(2), 257-301. doi: 10.1037/a0018301

Park, C. L., Chmielewski, J., \& Blank, T. O. (2010). Post-traumatic growth: finding positive meaning in cancer survivorship moderates the impact of intrusive thoughts on adjustment in younger adults. Psycho-Oncology, 19(11), 1139-1147. doi: 10.1002/pon. 1680

Park, C. L., Riley, K. E., \& Snyder, L. B. (2012). Meaning making coping, making sense, and post-traumatic growth following the 9/11 terrorist attacks. The Journal of Positive Psychology, 7(3), 198-207. doi: 10.1080/17439760.2012.671347

Pasupathi, M. (2001).The social construction of the personal past and its implications for adult development.Psychological Bulletin, 127(5), 651-672. doi: 10.1037/00332909.127.5.651

Pasupathi, M. (2012). Making meaning for the good life: A commentary on the special issue. Memory.doi: 10.1080/09658211.2012.744843 
Pasupathi, M., \& Hoyt, T. (2009). The development of narrative identity in late adolescence and emergent adulthood: The continued importance of listeners. Developmental Psychology, 45(2), 558-574. doi: 10.1037/a0014431

Pasupathi, M., \& Mansour, E. (2006).Adult age differences in autobiographical reasoning in narratives.Developmental Psychology, 42(5), 798-808. doi: 10.1037/00121649.42.5.798

Pasupathi, M., Mansour, E., \& Brubaker, J. R. (2007). Deveoping a life story: Constructing relations between self and experience in autobiographical narratives. Human Development, 50(2-3), 85-110. doi: 10.1159/000100939

Pavot, W., \& Diener, E. (1993). Review of the Satisfaction With Life Scale. Psychological Assessment, 5(2), 164-172. doi: 10.1037/1040-3590.5.2.164

Pavot, W., Diener, E., Colvin, R., \& Sandvik, E. (1991). Further validation of the Satisfaction With Life Scale: Evidence for the cross-method convergence of well-being measures. Journal of Personality Assessment, 57(1), 149-161. doi: 10.1207/s15327752jpa5701_17

Pennebaker, J. W. (1993). Putting stress into words: Health, linguistic, and therapeutic implications. Behaviour Research and Therapy, 31(6), 539-548. doi: 10.1016/00057967(93)90105-4

Pennebaker, J. W. (1997). Writing about emotional experiences as a therapeutic process.Psychological Science, 8(3), 162-166. doi: 10.1111/j.14679280.1997.tb00403.x

Pennebaker, J. W., \& Francis, M. E. (1996). Cognitive, emotional, and language processes in disclosure. Cognition \& Emotion, 10(6), 601-626. doi: 10.1080/026999396380079

Pennebaker, J. W., Mayne, T. J., \& Francis, M. E. (1997).Linguistic predictors of adaptive bereavement.Journal of Personality and Social Psychology, 72(4), 863-871. doi: 10.1037//0022-3514.72.4.863 
Pennebaker, J. W., \& Seagal, J. D. (1999).Forming a story: The health benefits of narrative.Journal of Clinical Psychology, 55(10), 1243-1254. doi: 10.1002/(SICI)1097-4679(199910)55:10<1243::AID-JCLP6>3.0.CO;2-N

Peterson, C. (1991). The meaning and measurement of explanatory style.Psychological Inquiry, 2(1), 1-10. doi: 10.1207/s15327965pli0201_1

Peterson, C., Schulman, P., Castellon, C., \& Seligman, M. E. P. (1992). CAVE: Content analysis of verbatim explanations. In C. P. Smith (Ed.), Motivation and personality: Handbook of thematic content analysis (pp. 383-392). New York: Cambridge University Press.

Peterson, C., \& Seligman, M. E. (1984). Causal explanations as a risk factor for depression: Theory and evidence. Psychological Review, 91(3), 347-374. doi: 10.1037/0033295X.91.3.347

Peterson, C., Semmel, A., von Baeyer, C., Abramson, L. Y., Metalsky, G. I., \& Seligman, M. E. (1982).The Attributional Style Questionnaire.Cognitive Therapy and Research, 6(3), 287-300. doi: 10.1007/BF01173577

Pillemer, D. B. (1998).Momentous events, vivid memories. Cambridge, Massachusetts: Harvard University Press.

Pillemer, D. B. (2001).Momentous events and the life story.Review of General Psychology, 5(2), 123-134. doi: 10.I037//1089-2680.5.2.123

Pincus, D. B., \& Friedman, A. G. (2004). Improving children's coping with everyday stress: transporting treatment interventions to the school setting. Clinical Child and Family REview, 7(4), 223-240. doi: 10.1007/s10567-004-6087-8

Preacher, K. J., Rucker, D. D., \& Hayes, A. F. (2007). Addressing moderated mediation hypotheses: Theory, methods, and prescriptions. Multivariate Behavioral Research, 42(1), 185-227. doi: 10.1080/00273170701341316

Prochaska, J. O., Wright, J. A., \& Velicer, W. F. (2008). Evaluating theories of health behavior change: A hierarchy of criteria applied to the transtheoretical model. Applied 
Psychology: An International Review, 57(4), 561-588.doi: 10.1111/j.14640597.2008.00345.x

Rapee, R. M., \& Heimberg, R. G. (1997).A cognitive-behavioral model of anxiety in social phobia.Behaviour Research and Therapy, 35(8), 741-756. doi: 10.1016/S00057967(97)00022-3

Reardon, J. M., \& Williams, N. L. (2007). The specificity of cognitive vulnerabilities to emotional disorders: Anxiety sensitivity, looming vulnerability and explanatory style. Journal of Anxiety Disorders, 21(5), 625-643. doi: 10.1016/j.janxdis.2006.09.013

Reese, E., Haden, C. A., \& Fivush, R. (1993). Mother-child conversations about the past: Relationships of style and memory over time. Cognitive Development, 8(4), 403-430. doi: 10.1016/S0885-2014(05)80002-4

Reese, E., Haden, C. A., \& Fivush, R. (1996). Mothers, fathers, daughters, sons: Gender differences in autobiographical reminiscing. Research on Language and Social Interaction, 29(1), 27-56. doi: 10.1207/s15327973rlsi2901_3

Rimes, K. A., \& Watkins, E. R. (2005).The effects of self-focused rumination on global negative self-judgements in depression.Behaviour Research and Therapy, 43(12), 1673-1681. doi: 10.1016/j.brat.2004.12.002

Riskind, J. H., \& Alloy, L. B. (2006). Cognitive vulnerability to psychological disorders: Overview of theory, design, and methods. Journal of Social and Clinical Psychology, 25(7), 705-725. doi: 10.1521/jscp.2006.25.7.705

Romens, S. E., MacCoon, D. G., Abramson, L. Y., \& Pollak, S. D. (2011). Cognitive style moderates attention to attribution-relevant stimuli. Cognitive Therapy and Research, 35(2), 134-141. doi: 10.1007/s10608-010-9345-8

Rood, L., Roelofs, J., Bogels, S. M., \& Arntz, A. (2012). The effects of experimentally induced rumination, positive reappraisal, acceptance, and distancing when thinkingabout a stressful event on affect states in adolescents. Journal of Abnormal Child Psychology, 40(1), 73-84. doi: 10.1007/s10802-011-9544-0 
Ross, M., \& Wilson, A. E. (2002). It feels like yesterday: Self-esteem, valence of personal past experiences, and judgements of subjective distance. Journal of Personality and Social Psychology, 82(5), 792-803. doi: 10.1037//0022-3514.82.5.792

Ross, M., \& Wilson, A. E. (2002). It feels like yesterday: Self-esteem, valence of personal past experiences, and judgments of subjective distance. Journal of Personality and Social Psychology, 82(5), 792-803. doi: 10.1037//0022-3514.82.5.792

Ross, M. J., Mueller, J. H., \& De La Torre, M. (1986).Depression and trait distinctiveness in the self-schema.Journal of Social and Clinical Psychology, 4(1), 46-59. doi: 10.1521/jscp.1986.4.1.46

Rubin, D. C., Berntsen, D., \& Hutson, M. (2009). The normative and the personal life: Individual differences in life scripts and life story events among USA and Danish undergraduates. Memory, 17(1), 54-68. doi: 10.1080/09658210802541442

Rubin, D. C., \& Siegler, I. (2004). Facets of personality and the phenomenology of autobiographical memory.Applied Cognitive Psychology, 18(7), 913-930.doi: 10.1002/acp.1038

Runyon, M. K., \& Kenny, M. C. (2002).Relationship of attributional style, depression, and posttrauma distress among children who suffered physical or sexual abuse.Child Maltreatment, 7(3), 254-264. doi: 10.1177/1077559502007003007

Ryan, R. M., \& Deci, E. L. (2001). On happiness and human potentials: A review of research on hedonic and eudaimonic well-being. Annual Review of Psychology, 52, 141-166. doi: 10.1146/annurev.psych.52.1.141

Ryff, C. D., \& Keyes, C. L. M. (1995). The structure of psychological well-being revisited. Journal of Personality and Social Psychology, 69(4), 719-727. doi: 10.1037/00223514.69.4.719

Ryff, C. D., \& Singer, B. (1996). Psychological well-being: Meaning, measurement, and implications for psychotherapy research. Psychotherapy and Psychosomatics, 65(1), 14-23. doi: 10.1159/000289026 
Sanjuán, P., \& Magallares, A. (2009).A longitudinal study of the negative explanatory style and attributions of uncontrollability as predictors of depressive symptoms.Personality and Individual Differences, 46(7), 714-718. doi: 10.1016/j.paid.2009.01.030

Sedikides, C., \& Brewer, M. B. (2001).Individual self, relational self, collecctive self. Philadelphia: Psychology Press.

Segal, Z. V., Hood, J. E., Shaw, B. F., \& Higgins, E. T. (1998). A structural analysis of the self-schema construct in major depression. Cognitive Therapy and Research, 12(5), 471-485. doi: 10.1007/BF01173414

Singer, J. A. (2004). Narrative Identity and Meaning Making Across the Adult Lifespan: An Introduction. Journal of Personality, 72(3), 437-459. doi: 10.1111/j.00223506.2004.00268.x

Singer, J. A., \& Bluck, S. (2001). New perspectives on autobiographical memory: the integration of narrative processing and autobiographical reasoning. Review of General Psychology, 5(2), 91-99. doi: 10.I037//1089-2680.5.2.91

Singer, J. A., \& Moffitt, K. H. (1991-1992). An experimental investigation of specificity and generality in memory narratives.Imagination, Cognition and Personality, 11(3), 233257.

Singer, J. A., \& Salovey, P. (1993).The remembered self: Emotion and memory inpersonality. New York: Free Press.

Smyth, J. M., Hockemeyer, J. R., \& Tulloch, H. (2008). Expressive writing and posttraumatic stress disorder: Effects on trauma symptoms, mood states, and cortisol reactivity. British Journal of Health Psychology, 13(1), 85-93.

doi: 10.1348/135910707X250866

Steger, M., Oishi, S., \& Kashdan, T. (2009). Meaning in life across the life span: Levels and correlates of meaning in life from emerging adulthood to older adulthood. The Journal of Positive Psychology: Dedicated to furthering research and promoting good practice, 4(1), 43-52. doi: 10.1080/17439760802303127 
Steger, M., Oishi, S., \& Kesebir, S. (2011). Is a life without meaning satisfying? The moderating role of the search for meaning in satisfaction with life judgments,.The Journal of Positive Psychology, 6(3), 173-180. doi: 10.1080/17439760.2011.569171

Steger, M. F., Frazier, P., Oishi, S., \& Kaler, M. (2006). The meaning in life questionnaire: Assessing the presence of and search for meaning in life. Journal of Counseling Psychology, 53(1), 80-93.doi: 10.1037/0022-0167.53.1.80

Steger, M. F., \& Kashdan, T. (2007).Stability and specificity of meaning in life and life satisfaction over one year.Journal of Happiness Studies, 8(2), 161-179. doi: $10.1007 / \mathrm{s} 10902-006-9011-8$

Stein, N., Folkman, S., Trabasso, T., \& Richards, T. A. (1997). Appraisal and goal processes as predictors of psychological well-being in bereaved caregivers. Journal of Personality and Social Psychology, 72(4), 872-884. doi: 10.1037/0022-3514.72.4.872

Sutin, A. R. (2008). Autobiographical memory as a dynamic process: Autobiographical memory mediates basic tendencies and characteristic adaptations. Journal of Research in Personality, 42, 1060-1066. doi: 10.1016/j.jrp.2007.10.002

Sutin, A. R., Costa, P. T., Wethington, E., \& Eaton, W. (2010). Perceptions of stressful life events as turning points are associated with self-rated health and psychological distress. Anxiety, Stress \& Coping: An International Journal, 23(5), 479-492. doi: $10.1080 / 10615800903552015$

Sutin, A. R., \& Robins, R. W. (2007). Phenomenology of autobiographical memories: The memory experiences questionnaire. Memory, 15(4), 390-411. doi: $10.1080 / 09658210701256654$

Sutin, A. R., \& Robins, R. W. (2010).Correlates and phenomenology of first and third person memories.Memory, 18(6), 625-637. doi: 10.1080/09658211.2010.497765

Sutherland, K., \& Bryant, R. (2005).Self-defining memories in post-traumatic stress disorder.British Journal of Clinical Psychology, 44(4), 591-598. doi: 10.1348/014466505X64081 
Swales, M. A., Williams, J. M. G., \& Wood, P. (2001).Specificity of autobiographical memory and mood disturbance in adolescents.Cognition and Emotion, 15(3), 321331. doi:10.1080/0269993004200132

Tavernier, R., \& Willoughby, T. (2012). Adolescent turning points: the association between meaning-making and psychological well-being. Developmental Psychology, 48(4), 1058-1068. doi: 10.1037/a0026326

Taylor, S. E., \& Brown, J. D. (1988). Illusion and well-being: A social psychological perspective on mental health. Psychological Bulletin, 103(2), 193-210. doi: 10.1037/0033-2909.103.2.193

Teasdale, J. D., \& Cox, S. G. (2001). Dysphoria: Self-devaluative and affective components in recovered depressed patients and never depressed controls. Psychological Medicine, 31(7), 1311-1316. doi: 10.1017\}S003329170100424X

Tedeschi, R. G., \& Calhoun, L. G. (1996). The posttraumatic growth inventory: Measuring the positive legacy of trauma. Journal of Traumatic Stress, 9(3), 455-471. doi: $10.1002 /$ jts. 2490090305

Tedeschi, R. G., \& Calhoun, L. G. (2004). Posttraumatic Growth: Conceptual foundations and empirical evidence.Psychological Inquiry,15(1), 1-18. doi: 10.1207/s15327965pli1501_01

Terry, W. S., \& Horton, G. P. (2007-2008). A comparison of self-rated emotion in field and observer memory perspectives.Imagination, Cognition and Personality, 27(1), 27-35. doi: 10.2190/IC.27.1.c

Thorne, A. (2000). Personal Memory Telling and Personality Development.Personality and Social Psychology Review, 4(1), 45-56. doi: 10.1207/S15327957PSPR0401_5

Thorne, A., McLean, K. C., \& Lawrence, A. M. (2004). When remembering is not enough: Reflecting on self-defining memories in late adolescence. Journal of Personality, 72(3), 513-542. doi: 10.1111/j.0022-3506.2004.00271.x 
Treynor, W., Gonzalez, R., \& Nolen-Hoeksema, S. (2003). Rumination reconsidered: A psychometric analysis. Cognitive Therapy and Research, 27(3), 247-259. doi: 10.1023/A:1023910315561

Trzesniewski, K. H., Donnellan, M. B., Moffitt, T. E., Robins, R. W., Poulton, R., \& Caspi, A. (2006). Low self-esteem during adolescence predicts poor health, criminal behavior, and limited economic prospects during adulthood. Developmental Psychology, 42(2), 381-390. doi:10.1037/0012-1649.42.2.381

Vignoles, V. L., Schwartz, S. J., \& Luyckx, K. (2011). Introduction: Toward an Integrative View of Identity. In S. J. Schwartz, K. Luyckx \& V. L. Vignoles (Eds.), Handbook of Identity Theory and Research: Volume 1 Structures and Processes (pp. 99-115). New York: Springer.

Vleioras, G., \& Bosma, H. A. (2005). Are identity styles important for psychological wellbeing? Journal of Adolescence, 28(3), 397-409. doi: 10.1016/j.adolescence.2004.09.001

Ward, T. (2012). Narrative identity and forensic psychology: A commentary on Youngs and Canter. Legal and Criminological Psychology, 17(2), 250-261. doi: 10.1111/j.20448333.2011.02028.x

Waterman, A. S. (1982). Identity development from adolescence to adulthood: An extension of theory and a review of research. Developmental Psychology, 18(3), 341-358. doi: $10.1037 / 0012-1649.18 .3 .341$

Watkins, E. R. (2009). Depressive rumination and co-morbidity: Evidence for brooding as a transdiagnostic process. Journal of Rational-Emotive and Cognitive Behaviour Therapy, 27(3), 160-175. doi: 10.1007/s10942-009-0098-9

Watkins, E., Teasdale, J.D., (2001) Rumination and overgeneral memory in depression: effects of self-focus and analytic thinking. Journal of Abnormal Psychology, 110(2), 353 - 357. doi: 10.1037/0021-843X.110.2.333

Webster, J., \& Tiggemann, M. (2003). The relationship between women's body satisfaction and self-image across the life span: The role of cognitive control.The Journal of Genetic Psychology, 164(2), 241-252. doi: 10.1080/00221320309597980 
Webster, J. D., \& McCall, M. E. (1999). Reminiscence functions across adulthood: a replication and extension. Journal of Adult Development, 6(1), 73-85. doi: 10.1023/A:1021628525902

Welburn, K., Coristine, M., Dagg, P., Pontefract, A., \& Jordan, S. (2002). The Schema Questionnaire-short form: Factor analysis and relationship between schemas and symptoms. Cognitive Therapy and Research, 26(4), 519-530. doi: 10.1023/A:1016231902020

Whitbourne, S. K., Sneed, J. R., \& Sayer, A. (2009). Psychosocial development from college through midlife: A 34-year sequential study. Developmental Psychology, 45(5), 13281340. doi: 10.1037/a0016550

Williams, J. M. G., Barnhofer, T., Crane, C., Herman, D., Raes, F., Watkins, E., \& Dalgleish, T. (2007).Autobiographical memory specificity and emotional disorder. Psychological Bulletin, 133(1), 122-148. doi:10.1037/0033-2909.133.1.122

Wilson, A. E., Gunn, G. R., \& Ross, M. (2009).The role of subjective time in identity regulation.Applied Cognitive Psychology, 23(8).doi: 10.1002/acp.1617

Wilson, A. E., \& Ross, M. (2001). From chump to champ: People's appraisals of their past and present selves. Journal of Personality and Social Psychology, 80(4), 572-584. doi: 10.1037//0022-3514.80.4.572

Wilson, A. E., \& Ross, M. J. (2003). The identity function of autobiographical memory: Time is on our side. Memory, 11(2), 137-149. doi: 10.1080/741938210

Wilson, Hayes, Gregg, \& Zettle, 2001).Psychopathology and Psychotherapy. In S. C. Hayes, D. Barnes-Holmes, \& B. Roche (Eds.), Relational Frame Theory: A Post-Skinnerian Account of Human Language and Cognition (pp 129-139). New York:Plenum.

Woodward, C., \& Joseph, S. (2003). Positive change processes and post-traumatic growth in people who have experienced childhood abuse: Understanding vehicles of change. Psychology and Psychotherapy: Theory, Research and Practice, 76(3), 267-283. doi: 10.1348/147608303322362497 
Wright, J. C., \& Mischel, W. (1987). A conditional approach to dispositional constructs: The local predictability of social behavior. Journal of Personality and Social Psychology, 53(6), 1159-1177. doi: 10.1037/0022-3514.53.6.1159

Zaman, W.\& Fivush, R. (2011).When my mom was a little girl...: Gender differences in adolescents' intergenerational and personal narratives.Journal of Research on Adolescence. 21(3), 703-716.DOI: 10.1111/j.1532-7795.2010.00709.x

Zika, S., \& Chamberlain, K. (1992).On the relation between meaning in life and psychological well-being.British Journal of Psychology, 83(1), 133-145. doi: 10.1111/j.2044-8295.1992.tb02429.x 